\title{
Development of new oil/water partitioning tracers for the determination of residual oil saturation in the inter-well region of water-flooded reservoirs
}

Mario Helder Lopes da Silva

\author{
Thesis submitted in fulfilment of \\ the requirements for the degree of \\ PHILOSOPHIAE DOCTOR \\ $(\mathrm{PhD})$

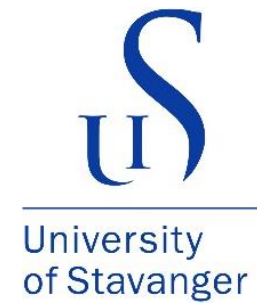

Faculty of Science and Technology Institute of Energy Resources 2021 
University of Stavanger

NO-4036 Stavanger

NORWAY

Www.uis.no

C2021 Mario Helder Lopes da Silva

ISBN: 978-82-7644-994-5

ISSN: $1890-1387$

PhD: Thesis UiS No. 578 
To my family,

Sofia, Maria João \& Francisca 


\section{Acknowledgements}

Albert Einstein is credited to have said "the value of achievement lies in the achieving." This is true for everything in life, and I feel very lucky and thankful for all the people I worked with in the path leading to this thesis. It is also their achievement, as it would not be possible without them. I learned and was trained by the best in the field, who also showed me that an open-mind, humility, and hard-work pave the way forward. I would like to thank my supervisors, Professor Tor Bjørnstad and Professor Svein Magne Skjæveland. Thank you, Tor, for all the insights, trust, freedom, patience, and advice! You truly are a role model, and I will be happy if I become half of what you are, both as a researcher and as a person. I could not have done this without you! Thank you Svein for all the kind and motivating words, for the example, and for the rigor with flexibility. This would not happen without you!

I want to thank the team of the "Tracer Technology department" at IFE, particularly Sissel Opsahl Viig for sharing her knowledge, time, patience, and good humour, as well as for the help in disassembling and assembling instrumentation! Per Arne Hubred, one of the best humoured and cleverest engineers I ever met, who made the "magic happen" whenever experimental needs would hit a wall. Are Haugen, for all the trouble and help in welcoming me to a new workplace and a new country. Laura Ferrando-Climent, for all the small talk in coffee breaks, confidences, incentive, the friendship, and the sharing of insights about good scientific work. A special thank you to Helge Stray, my office colleague, "informal supervisor", for all the fruitful discussions and exchange of ideas, reviews, motivation, friendship, and help with personal and logistic issues upon my arrival in Norway.

I would also like to thank the National IOR Centre of Norway for employing me and all its fantastic members, past and present colleagues at UiS, IFE and NORSE, especially Mahmoud Ould Metidji for the fantastic comradeship, friendship, and collaboration, Thomas Brichart 
for introducing me to the "nano-world" and for the comradeship, Jaspreet Singh Sachdeva for the optimism, motivation, and the insights about pore-scale phenomena, Eystein Opsahl for the warmth, good humour, good discussions, and excellent collaboration. Additionally, I want to thank Aksel Hiorth, Jan Ludvig Vinningland, Merete Madla Vadland, Randi Valestrand, for all the gatherings, discussions, and insights always with lots of fun! Many more are left out, each with valuable contributions. Working with the National IOR Centre of Norway was an exciting and challenging experience, filled with excellent scientific discussions, exchange of ideas and fun, without forgetting our industry partners and their valuable input.

Finally, my biggest thank you to my family! To Sofia, for the strength and constant support throughout all the challenges I faced even while facing her own! None of my achievements would be possible without you as my partner and inspiration! To Maria João and Francisca, for being the most wonderful girls in the world and giving me the privilege of learning so much from you! The way you faced the challenge of moving to a new country, your development, and the people you are becoming inspire and make me proud every day! 


\section{Summary}

Most of the hydrocarbon-rich large unexplored basins are located in remote and/or highly environmentally sensitive regions. As more and more oilfields reach maturity at the end of the secondary stage of recovery, while on average more than $50 \%$ of the original reserves of hydrocarbons are left in place, the future of oil production on the Norwegian Continental shelf (NCS) will increasingly rely on IOR projects to face the demand. A partitioning inter-well tracer test (PITT) is a type of tracer test that measures the water contactable saturation of immobile oil ( $\left.\mathrm{S}_{\mathrm{OR}}\right)$ in the inter-well region of water flooded reservoirs. Knowledge about $\mathrm{S}_{\mathrm{OR}}$ in the swept volumes between injector/producer pairs is a key parameter for the design and evaluation of IOR projects.

The PITT explores the lag in production experienced by an oil/water partitioning tracer relatively to a passive water tracer which directly correlates to $S_{\mathrm{OR}}$. This principle was introduced to the industry in 1971 and relied on the use of molecules successfully applied in hydrology and/or labelled with radioactive nuclides for easier detection. The conditions encountered in oil reservoirs, particularly in deep oil formations, are significantly harsher than in the near surface subsoil. Thus, several unsuccessful inter-well tracer tests resulted from a poor selection of the tracer compounds used at that time because of insufficient knowledge about their behaviour under typical reservoir conditions. Much work has been done to improve the original concept of the PITT regard its deployment and interpretation. However, little effort has been put in place to establish a systematic procedure for selecting, testing and describing the dynamic behaviour of the substances used as oil/water partitioning tracers. Thus, this thesis aims to present a methodology for selection and testing of new PITT tracer candidates, with the results and findings of its application to a selected group of molecules. 
The methodology presented here starts by describing the base requirements for selection of new oil/water partitioning tracer candidates. Additionally, guidelines for testing and qualification are presented. There are several steps in the qualification procedure. These can be divided into static stability experiments, development of analytical methods for laboratory samples, development of analytical methods for identification and quantification of the stable molecules in real produced waters, characterisation of the oil/water partition coefficient $(\mathrm{K})$ of the molecules, and dynamic flooding experiments using cores of consolidated sedimentary rock. Following this method, step by step, 16 molecules from 4 different chemical "families" were selected and tested for qualification as new oil/water partitioning tracers. The static stability experiments evaluated the thermal stability of the PITT tracer candidates, in the absence and presence of typical reservoir rock materials, different $\mathrm{pH}$ conditions, and elevated salinity up to 150 ${ }^{\circ} \mathrm{C}$ for 12 weeks contact time. Ultra-performance liquid chromatography (UPLC) coupled with ultra-violet detection (UV) and high-resolution mass spectrometry (HRMS), and gas chromatography (GC) coupled with flame ionisation detection (FID) were the techniques used to follow the concentration of the PITT tracer candidates along the 12 weeks of experiment. UPLC-HRMS was used to try to obtain relevant information to describe the observed phenomena. The static stability experiments proved that only 5 of the 16 tested compounds were stable for 12 weeks up to $150{ }^{\circ} \mathrm{C}$. Two additional compounds were stable for the same period up to $125^{\circ} \mathrm{C}$. This is sufficient to allow for their use in most oilfields, and they were thus included as possible inter-well PITT tracers. These experiments also revealed dramatic interactions between some of the studied molecules and clay minerals of undefined nature, as well as thermally driven degradation of the candidates that is well described by a pseudo-first order kinetic model. The latter two findings open the possibility of using tracers to retrieve information about temperature and geochemistry/mineralogy in the inter-well region, though the latter requires further development. 
A method based on sequential direct immersion (DI) headspace (HS) solid-phase microextraction (SPME) proved effective as analytical sample pre-treatment followed by GC-MS/MS for analysis of the PITT tracer candidate concentrations in real production waters. The DI-HSSPME-GC-MS/MS method allows for quantification of the stable molecules investigated in low $\mathrm{ng} / \mathrm{L}$ concentrations and introduces significant improvements in comparison to the commonly used methodologies for analysis of tracers in produced waters: it requires just $5 \mathrm{~mL}$ of sample and eliminates the need for use of organic solvents in the laboratory. Furthermore, sample processing times are significantly reduced as the cleaning/concentration step becomes much faster. This is of utmost importance for a PITT, as several hundreds of samples are analysed in these examinations.

The characterisation of the K-values of the stable PITT tracer candidates revealed that they will likely vary along the volume swept between injector/producer pairs. $\mathrm{K}$ is influenced primarily by the ionic strength of the aqueous phase and composition of the hydrocarbon phase, and to a smaller extent by temperature $\mathrm{T}$. The influence of temperature can, however, be very relevant: The K-value is used in the calculation of $\mathrm{S}_{\mathrm{OR}}$ together with the times of arrival of the different tracers using the same landmark of their respective production curves (i.e., the theoretically most correct is the first moments of the curves). It is likely that the temperature varies between injector and producer well-pairs. The variation of the $\mathrm{K}$-value as function of $\mathrm{T}$ needs to be accounted for to determine accurate $S_{\text {OR }}$ values. Variations of the ionic strength will lead to even bigger variations of the K-value independently of the valency of the ions present in the aqueous phase. The experiments performed also confirmed the constant and reversible equilibrium distribution of the oil/water tracer candidates between hydrocarbon and aqueous phases, as well as their suitability for use on most oilfields of the NCS.

The flooding experiments were performed on sandstone and chalk cores prepared both to pure water saturation and to residual oil saturation, $\mathrm{S}_{\mathrm{OR}}$. Residence time distribution analysis (RTD) was used to interpret the 
production curves. These experiments proved that the PITT tracer candidates behave as water tracers in the absence of hydrocarbons, with no significant difference encountered between their production curves and the ones obtained from the reference water tracer (tritiated water). $S_{\text {OR }}$ was determined for two different mass recovery landmarks in each of the experiments for all partitioning tracers using the $\mathrm{K}$-values previously determined in the experiments for characterisation of the $\mathrm{K}$ values. Good agreement between all Sor values measured by the tracer candidates and the values determined by a balance to the oil used to prepare the cores was encountered. This is also valid when $S_{\text {OR }}$ measured by the tracers is compared to the value obtained by the differences in water flooded pore volumes measured by tritiated water.

The methodology presented and applied in this thesis produced 7 new oil/water partitioning tracers ready to be used in oil fields with low probability of failure. The findings and observations presented here can be incorporated into reservoir models to obtain more accurate data from PITTs, and consequently better reservoir description. Furthermore, the reinjection of produced waters will lead to contaminations of the interwell region with tracers used there. Thus, the present study offers guidelines and methods for the development of new tracers. The oil industry, service companies, and other researchers working with tracer technology will be the primary beneficiaries of this study, that will hopefully contribute to disseminate the use of PITTs by the industry. This technology has a large potential to contribute to a future efficient and profitable oil production. 


\section{List of publications}

\section{Paper I:}

Mário Silva, Helge Stray, Tor Bjørnstad (2017)

Studies on New Chemical Tracers for Determination of Residual Oil Saturation in the Inter-Well Region.

SPE Oil and Gas Symposium 2017, Oklahoma City, Oklahoma, USA.

\section{Paper II:}

Mário Silva, Helge Stray, Tor Bjørnstad (2017)

New Potential Tracer Compounds for Inter-well SOR Determination Stability at Reservoir Conditions.

EAGE 19th European Symposium on Improved Oil Recovery, Stavanger, Norway.

\section{Paper III:}

Mário Silva, Helge Stray, Tor Bjørnstad (2018)

Stability assessment of PITT tracer candidate compounds: the case of benzyl alcohols.

Journal of Petroleum Science and Engineering 167, 517-523.

\section{Paper IV:}

Mário Silva, Helge Stray, Tor Bjørnstad (2019)

Stability assessment of PITT tracer candidate compounds: the case of pyrazines.

Journal of Petroleum Science and Engineering 182, 106269. 


\section{Paper V:}

Mário Silva, Helge Stray, Mahmoud Ould Metidji, Tor Bjørnstad (2021)

Thermal stability and interactions with sedimentary rocks under typical reservoir conditions of selected pyridines investigated as PITT tracers.

Upstream Oil and Gas Technology 06, 100033.

\section{Paper VI:}

Mário Silva, Tor Bjørnstad (2020)

Determination of phase-partitioning tracer candidates in production waters from oilfields based on solid-phase microextraction followed by gas chromatography-tandem mass spectrometry.

Journal of Chromatography A 1629, 461508.

\section{Paper VII:}

Mário Silva, Helge Stray, Mahmoud Ould Metidji, Tor Bjørnstad (In review)

The partition coefficient of phase-partitioning compounds between hydrocarbon and aqueous phases: an experimental study.

Fuel. 


\section{List of additional papers}

Arun K. P. Selvam, Mahmoud Ould Metidji, Mario Silva, Alexander Krivokapic, Tor Bjørnstad (2020)

Thermo-Sensitive Nanogels for Targeted Tracer Release in Push and Pull Operations.

SPE Improved Oil Recovery Conference, Tulsa, Oklahoma, USA.

Mário Silva, Mahmoud Ould Metidji, Helge Stray, Tor Bjørnstad (2019)

Alkylpyrazines - from the "Dinner Table" to the Oilfield: A New Class of Partitioning Tracers.

EAGE $20^{\text {th }}$ European Symposium on Improved Oil Recovery, Pau, France.

Mahmoud Ould Metidji, Mário Silva, Alexander Krivokapic, Tor Bjørnstad (2019)

Synthesis and Characterization of a Reactive Fluorescent Tracer and its Possible Use for Reservoir Temperature's Data Collection.

EAGE $20^{\text {th }}$ European Symposium on Improved Oil Recovery, Pau, France.

Thomas Brichart, Mahmoud Ould Metidji, Mario Silva, Laura Ferrando-Climent, Tor Bjørnstad (2017)

New Fluorescent Tracers for SWCTT.

EAGE $19^{\text {th }}$ European Symposium on Improved Oil Recovery, Stavanger, Norway. 


\section{Conference contributions}

2020

Arun K. P. Selvam, Mahmoud Ould Metidji, Mario Silva, Alexander Krivokapic, Tor Bjørnstad. Thermo-Sensitive Nanogels for Targeted Tracer Release in Push and Pull Operations. SPE Improved Oil Recovery Conference, 2020.

2019

Mário Silva, Mahmoud Ould Metidji, Helge Stray, Tor Bjørnstad. Alkylpyrazines - A New Class of Partitioning Tracers? EAGE $20^{\text {th }}$ European Symposium on Improved Oil Recovery, 2019.

Mahmoud Ould Metidji, Mário Silva, Alexander Krivokapic, Tor Bjørnstad. Synthesis and Characterization of a Reactive Fluorescent Tracer and its Possible Use for Reservoir Temperature's Data Collection. EAGE $20^{\text {th }}$ European Symposium on Improved Oil Recovery, 2019.

Mário Silva, Helge Stray, Sissel O. Viig, Mahmoud Ould Metidji, Tor Bjørnstad. Revealing the hidden: Phase partitioning tracers can lead to pure SORcery. IOR Norway 2019.

Mahmoud Ould Metidji, Alexander Krivokapic, Mário Silva, Tor Bjørnstad, Arun Panner Selvam, Laurence Cathles III. Contribution to the Study on Nanoparticles as Oil-Reservoir' Tracers. IOR Norway 2019.

Arun Panner Selvam, Mahmoud Ould Metidji, Mário Silva, Sissel O. Viig, Merete Vadla Madland, Tor Bjørnstad. PhD-project: Nanoparticle tracers for petroleum reservoir studies. IOR Norway 2019. 
Mário Silva, Helge Stray, Tor Bjørnstad. The Influence of Key Reservoir Parameters on the Partition Coefficient of PITT Tracer Candidates. IOR Norway 2018.

Mário Silva, Mahmoud Ould Metidji, Helge Stray, Tor Bjørnstad. Non-Conservative Tracers for Temperature Assessment in the Inter-Well region of Oil Reservoirs. SPE Workshop: Improved Decision-Making Through Tracer Technology, 2018.

Mahmoud Ould Metidji, Alexander Krivokapic, Lawrence Cathles, Mário Silva, Tor Bjørnstad. Nanotracers intended for EOR-operations. SPE Workshop: Improved Decision-Making Through Tracer Technology, 2018.

2017

Mahmoud Ould Metidji, Serkan Kelesoglu, Mario Silva, Tor Bjørnstad. Laboratory assessment of nanotracers for oil reservoir characterization. C2E - Colloids and Complex fluids for Energies Systems understanding: from preparation to process design, 2017.

Mário Silva, Helge Stray, Tor Bjørnstad. PITT Tracer Candidates: Stability at Reservoir Conditions. IOR Norway 2017.

Mário Silva, Helge Stray, Tor Bjørnstad. Studies on New Chemical Tracers for Determination of Residual Oil Saturation in the Inter-Well Region. SPE Oklahoma City Oil and Gas Symposium, 2017.

2016

Mario Silva, Tor Bjørnstad. Solid-Phase Microextraction as Sample Preparation Technique in Tracer Technology. IOR Norway 2016. 
Thomas Brichart, Alexander Krivokapic, Mario Silva, Tor Bjørnstad. Can nano-fluorescent objects be used as reservoir tracers? IOR Norway 2016. 


\section{Table of Contents}

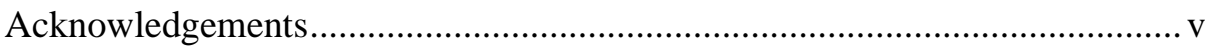

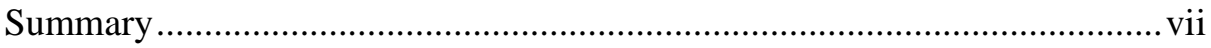

List of publications ………………..............................................................

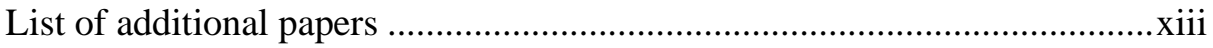

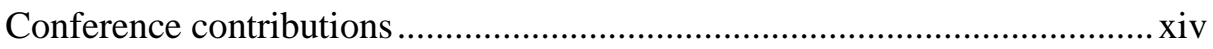

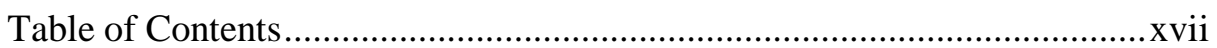

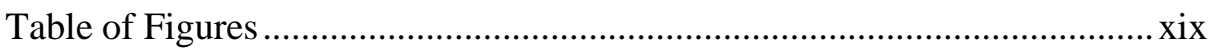

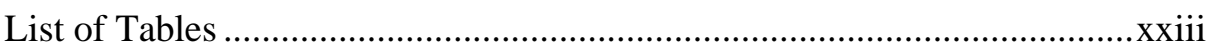

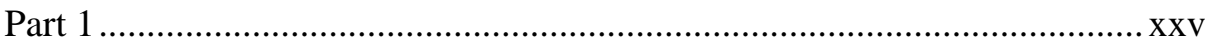

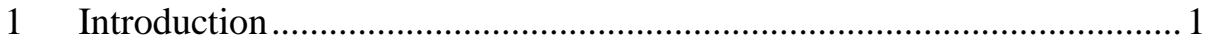

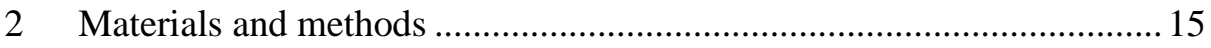

2.1 Selection of the partitioning tracer candidates .................................. 15

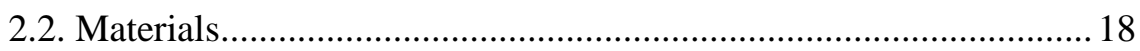

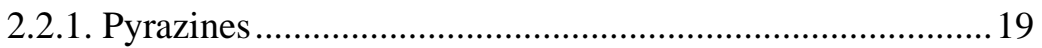

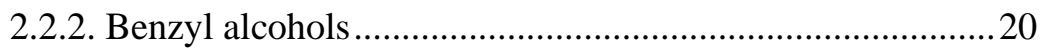

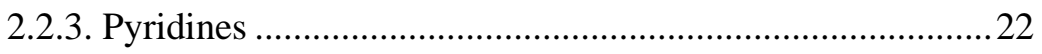

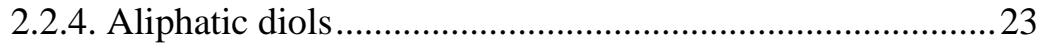

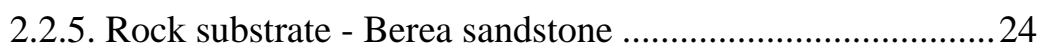

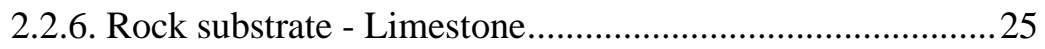

2.2.7. Rock substrate - Kaolinite....................................................26

2.2.8. Rock substrate - Stevns Klint chalk (Sigerslev member) ...26

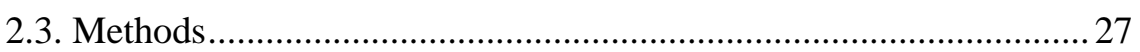

2.3.1. Analytical methods for laboratory samples $-\mathrm{I}$...................28

2.3.2. Analytical methods for laboratory samples - II....................36

2.3.3. Static stability experiments .................................................. 38

2.3.4. Analytical methods for field samples ................................. 41

2.3.5. Characterisation of the K-value ......................................... 42 


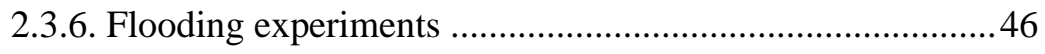

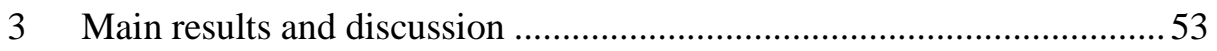

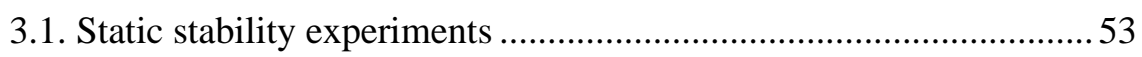

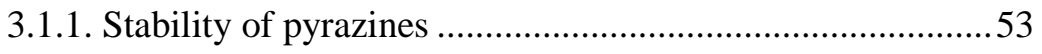

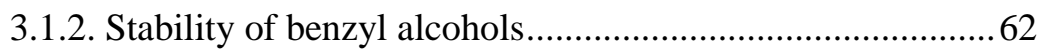

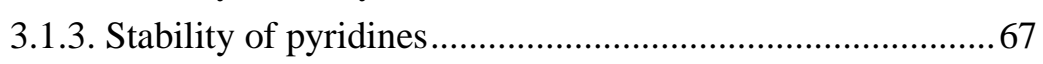

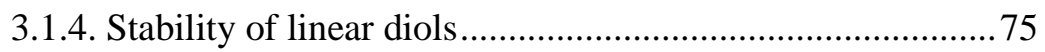

3.1.5. Summary of the stability experiments ................................ 77

3.2. Development of an analytical method for real field samples ............77

3.3. Evaluation of the K-value of the stable PITT tracer candidates....... 84

3.4. Dynamic flooding properties of the stable PITT tracer candidates.. 92

3.4.1. Flooding experiments in water saturated cores...................93

3.4.2. Flooding experiments in cores in $\mathrm{S}_{\mathrm{OR}}$ conditions.................98

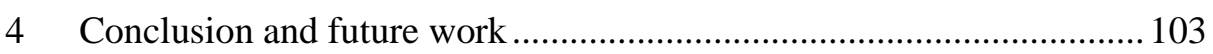

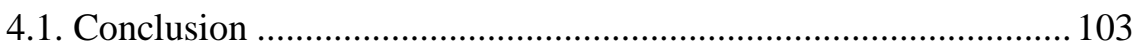

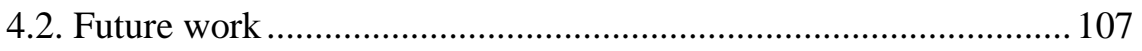

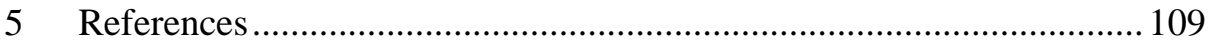

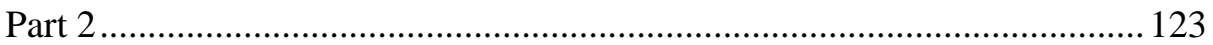

Paper I: Studies on New Chemical Tracers for Determination of Residual Oil Saturation in the Inter-Well Region.

Paper II: New Potential Tracer Compounds for Inter-well SOR Determination - Stability at Reservoir Conditions.

Paper III: Stability assessment of PITT tracer candidate compounds: the case of benzyl alcohols.

Paper IV: Stability assessment of PITT tracer candidate compounds: the case of pyrazines.

Paper V: Thermal stability and interactions with sedimentary rocks under typical reservoir conditions of selected pyridines investigated as PITT tracers. 
Paper VI: Determination of phase-partitioning tracer candidates in production waters from oilfields based on solid-phase microextraction followed by gas chromatography-tandem mass spectrometry.

Paper VII: The partition coefficient of phase-partitioning compounds between hydrocarbon and aqueous phases: an experimental study.

\section{Table of Figures}

Figure 1. Illustration of the principle of a PITT. When traveling through a hydrocarbon bearing medium, the phase-partitioning tracer (orange dots) is delayed relatively to the passive water tracer (blue dots). SOR between injector/producer well pairs can be determined from the tracer production curves.

Figure 2. Generic structure of fluorinated benzoic acids................................ 8

Figure 3. Structure and molecular mass (M) of the pyrazines selected as oil/water partitioning tracer candidates

Figure 4. Structure and molecular mass (M) of the benzyl alcohols selected as oil/water partitioning tracer candidates

Figure 5. Structure and molecular mass (M) of the pyridines selected as oil/water partitioning tracer candidates.

Figure 6. Structure and molecular mass (M) of the aliphatic diols selected as $\mathrm{oil} /$ water partitioning tracer candidates.

Figure 7. Example UPLC-UV chromatogram of a mixture of the 13 tracers analysable by the method developed at a concentration of 10 $\mathrm{mg} / \mathrm{L}$

Figure 8. Example GC-FID chromatogram of a mixture of the tracers analysable by the method developed at a concentration of 10 $\mathrm{mg} / \mathrm{L}$. Only 12HEXDIOL $\left(\mathrm{t}_{\mathrm{R}}=8,644 \mathrm{~min}\right)$ and 16HEXDIOL $\left(\mathrm{t}_{\mathrm{R}}=8,977 \mathrm{~min}\right)$ were determined by this method.

Figure 9. Example UPLC-UV chromatogram of a mixture of the relevant tracers analysable by the new method at a concentration of 10 $\mathrm{mg} / \mathrm{L}$. Retention times in minutes are given on the top of the peaks. See Table 6 for identification of the peaks

Figure 10. Picture of the samples tested inside one of thermal cabinets with a rotation mechanism. 41 
Figure 11. Simplified scheme of HTO analysis in a LSC instrument

Figure 12. Energy spectra of tritium in the form of tritiated water at two different activity concentrations: $82 \pm 4 \mathrm{~Bq} / \mathrm{L}$ (red curve) and $10 \pm 2 \mathrm{~Bq} / \mathrm{L}$ (blue curve), together with a background spectrum (yellow curve). 48

Figure 13. Scheme of the experimental setup used in the flooding experiments

Figure 14. RF values of 2,3-Dimethylpyrazine as function of time and temperature of incubation. (A) Initial pH 5.5; (B) Initial $\mathrm{pH} 7.1$;

(C) Initial $\mathrm{pH} 8.0$ and no rock substrate. 55

Figure 15. RF values of 2,6-Dimethylpyrazine as function of time and temperature of incubation. (A) Initial $\mathrm{pH} 5.5$; (B) Initial $\mathrm{pH} 7.1$; (C) Initial $\mathrm{pH} 8.0$ and no rock substrate. .56

Figure 16. RF values of 2-methoxypyrazine as function of time and temperature of incubation. (A) Initial pH 5.5; (B) Initial pH 7.1; (C) Initial $\mathrm{pH} 8.0$ and no rock substrate. .57

Figure 17. RF values of 2-chloropyrazine as function of time and temperature of incubation. (A) Initial pH 5.5; (B) Initial pH 7.1; (C) Initial $\mathrm{pH} 8.0$ and no rock substrate. 58

Figure 18. RF values of 2-fluoropyrazine as function of time and temperature of incubation. (A) Initial pH 5.5; (B) Initial pH 7.1; (C) Initial $\mathrm{pH} 8.0$ and no rock substrate. .59

Figure 19. Maximum differences encountered in $\mathrm{RF}$ values between experiments with rock substrate (rs) and experiments without rock substrate $\pm 2 \sigma$ in RF (t, T). (KA) kaolinite, (BS) Berea sandstone, (LS) limestone. (A) Initial pH 5.5; (B) Initial pH 7.1;

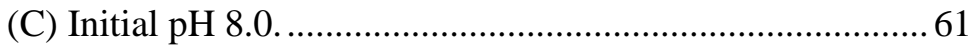

Figure 20. RF of 4-Chlorobenzyl alcohol and 2,6-Dichlorobenzyl alcohol as function of time and temperature of incubation. (A) 4Chlorobenzyl alcohol, (B) 2,6-Dichlorobenzyl alcohol. Initial experimental $\mathrm{pH} 7.1$, no rock substrate). 63

Figure 21. RF of 4-Methoxybenzyl alcohol and 3,4-Dimethoxybenzyl alcohol as function of time and temperature of incubation. (A) 4Methoxybenzyl alcohol, (B) 3,4-Dimethoxybenzyl alcohol. Initial experimental $\mathrm{pH} 7.1$, no rock substrate) .64 
Figure 22. RF of 4-hydroxybenzyl alcohol as function of time and temperature of incubation. Initial experimental $\mathrm{pH} 7.1$, no rock substrate. 65

Figure 23. Experimental vs predicted RF values of 4-hydroxybenzyl alcohol between 75 and $150{ }^{\circ} \mathrm{C}$. Uncertainty is not depicted for values under the lower analytical threshold $(\mathrm{RF}=0,05)$. 66

Figure 24. Maximum differences encountered in $\mathrm{RF}$ values between experiments with rock substrate (rs) and experiments without rock substrate $\pm 2 \times \sigma$ in RF (t, T). (KA) kaolinite, (BS) Berea sandstone, (LS) limestone, vs predicted RF values of 4hydroxybenzyl alcohol between 75 and $150{ }^{\circ} \mathrm{C}$. Uncertainty is not depicted for values under the lower analytical threshold (RF $=0,05)$.

Figure 25. RF of pyridine as function of time and temperature of incubation. (A) Initial $\mathrm{pH} 5.5$; (B) Initial $\mathrm{pH} 7.1$; (C) Initial $\mathrm{pH} 8.0$ and no rock substrate in any of the samples..................................... 68

Figure 26. RF of 2-hydroxy-6-methylpyridine as function of time and temperature of incubation. (A) Initial $\mathrm{pH} 5.5$; (B) Initial $\mathrm{pH} 7.1$; (C) Initial $\mathrm{pH} 8.0$ and no rock substrate in any of the samples. 69

Figure 27. RF of 3-hydroxypyridine as function of time and temperature of incubation. (A) Initial pH 5.5; (B) Initial pH 7.1; (C) Initial pH 8.0 and no rock substrate in any of the samples. .71

Figure 28. RF of 4-methoxypyridine as function of time and temperature of incubation. (A) Initial pH 5.5; (B) Initial pH 7.1; (C) Initial pH 8.0 and no rock substrate in any of the samples.

Figure 29. Maximum differences encountered in RF values between experiments with rock substrate (rs) and experiments without rock substrate $\pm 2 \times \sigma$ in $\mathrm{RF}(\mathrm{t}, \mathrm{T})$. (KA) kaolinite, (BS) Berea sandstone, (LS) limestone. (A) Initial pH 5.5; (B) Initial pH 7.1;

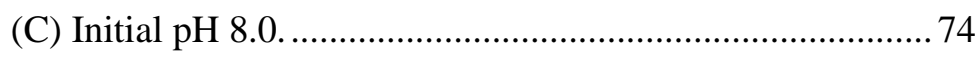

Figure 30. Maximum differences encountered in RF-values of 3hydroxypyridine between experiments with kaolinite (KA) and experiments without rock substrate (rs) as function of temperature and $\mathrm{pH}$. .75 
Figure 31. Remaining fraction of 1,2-hexanediol as a function of temperature after 12 weeks with and without rock substrates (A: initial $\mathrm{pH}$ 5.5; B: initial $\mathrm{pH} 8.0$ ).

Figure 32. Remaining fraction of 1,6-hexanediol as a function of temperature after 12 weeks with and without rock substrates (A: initial $\mathrm{pH}$ 5.5; B: initial $\mathrm{pH} 8.0$ ). .76

Figure 33. Geometric mean response area of the target compounds as function of the time and temperature of HS-SPME extraction after a fixed DI-SPME period of 5 minutes. 81

Figure 34. Reconstructed SRM chromatogram of produced water from Ekofisk $\mathrm{M}$ spiked at $1 \mu \mathrm{g} \mathrm{L}^{-1}$ with the transitions used for quantification of the PITT tracer candidates indicated. 82

Figure 35. K-values of pyridine $\pm \sigma(\mathrm{N}=3)$ as function of temperature, composition of the hydrocarbon phase and salinity. 86

Figure 36. K-values of 2,3-dimethylpyrazine $\pm \sigma(\mathrm{N}=3)$ as function of temperature, composition of the hydrocarbon phase and salinity. 86

Figure 37. K-values of 2,6-dimethylpyrazine $\pm \sigma(\mathrm{N}=3)$ as function of temperature, composition of the hydrocarbon phase and salinity

Figure 38. K-values of 4-methoxybenzyl alcohol $\pm \sigma(\mathrm{N}=3)$ as function of temperature, composition of the hydrocarbon phase and salinity.

Figure 39. K-values of 3,4-dimethoxybenzyl alcohol $\pm \sigma(\mathrm{N}=3)$ as function of temperature, composition of the hydrocarbon phase and salinity. 88

Figure 40. K-values of 4-chlorobenzyl alcohol $\pm \sigma(\mathrm{N}=3)$ as function of temperature, composition of the hydrocarbon phase and salinity. .88

Figure 41. K-values of 2,6-dichlorobenzyl alcohol $\pm \sigma(\mathrm{N}=3)$ as function of temperature, composition of the hydrocarbon phase and salinity. 89

Figure 42. K-values $\pm \sigma(\mathrm{N}=3)$ of the tested compounds in experiments with an aqueous phase containing divalent ions vs the $\mathrm{K}$-values in experiments with an aqueous phase containing only $\mathrm{NaCl}$ for the same ion strength. .91 
Figure 43. E ( $\mathrm{t}$ ) vs eluted mobile phase in the flooding experiments with a water-saturated sandstone core.

Figure 44. E ( $t$ ) vs eluted mobile phase in the flooding experiments with a water-saturated chalk core.

Figure 45. EN (t) of the PITT tracer candidates - EN ( $t$ ) of HTO vs eluted pore volume in the water saturated sandstone core.

Figure 46. EN (t) of the PITT tracer candidates - EN (t) of HTO vs eluted pore volume in the water saturated chalk core.

Figure 47. E (t) vs eluted mobile phase in the flooding experiments with the sandstone core in $\mathrm{S}_{\mathrm{OR}}$ conditions

Figure 48. E ( $t$ ) vs eluted mobile phase in the flooding experiments with the chalk core in $S_{\mathrm{OR}}$ conditions .99

\section{List of Tables}

Table 1 - Identification and relevant properties of the molecules selected as new oil/water partitioning tracer candidates

Table 2 - Steps and status of the methodology developed for qualification of new oil/water partitioning tracers for inter-well application. . 28

Table 3 - Retention time (tR), linear range, LOQ, intra-day and inter-day precision for each of the PITT tracer candidates analysed by the UPLC-UV based method.

Table 4 - Retention time $\left(\mathrm{t}_{\mathrm{R}}\right)$, linear range, intra-day and inter-day precision for both PITT tracer candidates analysed by the GC-FID based method. .35

Table 5 - Recovery at three different levels of concentration for each of the 16 PITT tracer candidates $(\mathrm{N}=5)$ (SPME-GC-FID was used to determine 12HEXDIOL and 16HEXDIOL and UPLC-UV for all other compounds). 36

Table 6 - Retention time $\left(t_{R}\right)$ for each of the PITT tracer candidates analysed by the new UPLC-UV based method.

Table 7 - Composition of the brine used in the experiments (from Paper III).

Table 8 - Salts, respective amount present, and I of the aqueous solutions of the studied compounds (Paper VII). .46 
Table 9 - Linearity, limits of quantification and precision of the DI-HS-SPMEGC-MS/MS evaluated at three concentrations.

Table 10 - Recoveries of HTO, the PITT tracer candidates and total pore volume calculated from each of their respective production curves.

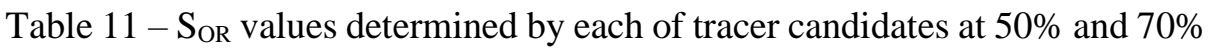
recovery in the sandstone core in $\mathrm{S}_{\mathrm{OR}}$ conditions

Table 12 - Sor values determined by each of tracer candidates at $50 \%$ and $70 \%$ recovery in the chalk core in $\mathrm{S}_{\mathrm{OR}}$ conditions. 101 
Part 1 


\section{Introduction}

Many oilfields on the Norwegian Continental Shelf (NCS) are mature (or reaching maturity) and this is reflected in the country's net oil production. Data from the Norwegian Petroleum Directorate (NPD) shows that the oil production from the NCS reached its peak in the year 2000 and exhibits a declining trend ever since (NPD, 2020). Furthermore, the residual oil saturation $\left(\mathrm{S}_{\mathrm{OR}}\right)$ after planned production cessation is often equal to or higher than $50 \%$ of the original oil in place. Enhanced oil recovery (EOR) techniques and carefully designed improved oil recovery (IOR) projects aiming for an efficient and profitable production of the remaining oil reserves will play an increasingly important part for the oil industry on the Norwegian continental shelf in the years to come. The design of such projects requires a thorough knowledge about the reservoirs. Therefore, reservoir characterisation techniques are critical for a new era of IOR-based oil production. One of the most important parameters for evaluation of the IOR potential is the residual oil saturation $\left(\mathrm{S}_{\mathrm{OR}}\right)$ in swept volumes between injector/producer well pairs. $\mathrm{S}_{\mathrm{OR}}$ is defined as the immobile oil remaining in place after secondary recovery (when maturity is reached). In water flooded reservoirs, maturity can be readily evaluated from logging of the water cut $(\geq 95 \%)$ in production wells. A partitioning inter-well tracer test (PITT) is tracer test that has the potential to measure $\mathrm{S}_{\mathrm{OR}}$ in the inter-well region of mature water flooded reservoirs. PITTs are, may be besides the 4D seismic, the only dynamic tool available to determine hydrocarbon saturation in the flooded areas of oil reservoirs and can be used to identify IOR targets, assess the efficiency of IOR projects or evaluate different EOR techniques in small scale field pilots. The fundamentals for the PITT were adopted from tracer tests used in hydrogeology to assess the contamination of soils by non-aqueous phase liquids (NAPLs) (Meinardus et al., 2002), discipline where they are still widely used. The introduction of the PITT principle to the oil industry 
took place in 1971 in a patent by C.E.J. Cooke (Cooke, 1971) registered by "Esso Production Research Company" in the USA. The basic concept behind a PITT is to treat a mature water flooded oil reservoir like a reverse-phase liquid chromatography column where the remaining hydrocarbons are the "stationary phase". The test relies on the simultaneous injection of one or more passive water and oil/water phasepartitioning tracers. The production of the tracers is then followed in one or more production wells to build tracer production curves. The partitioning tracer will exhibit a lag in production relatively to a passive one. This lag is dependent on the amount of hydrocarbons present along the travelled path. In water flooded oil reservoirs, passive water tracers and phase-partitioning tracers can be defined as follows (Leo et al., 1971):

- Passive water tracer: a substance that flows together with the injected water, traveling through the same channels and diffusing to same pores as it does, without any type of interaction with any of the other reservoir constituents (hydrocarbons and rocks).

- Oil/water partitioning tracer: a substance that behaves as a passive water tracer in the absence of hydrocarbons. When immiscible hydrocarbon phases and water become come in contact, the partitioning tracer exhibits an equilibrium distribution with real solutions in both phases.

The quotient between the equilibrium concentration of the tracer in the hydrocarbon and aqueous phases is the so-called oil/water partition coefficient (K-value). For a given system, the K-value is constant and independent of the initial concentration of the tracer or which of the two phases it was originally present in. Cooke's original work (Cooke, 1971) is based on two assumptions: i) the equilibrium distribution of the phasepartitioning tracer between the hydrocarbon and aqueous phase happens instantaneously when both phases become in contact, i.e., the kinetics of the distribution reaction is very fast; ii) the flow of the hydrocarbon phase is non-existent (it is stationary) or negligible in comparison to the aqueous phase. When these assumptions hold, (Deans, 1978) 
demonstrated that the ratio of the travelling times through the same flooded section of an oilfield between tracers $i$ and $j$, with oil/water partition coefficients $\mathrm{Ki}$ and $\mathrm{Kj}$ respectively, is given by:

$\frac{T_{j}}{T_{i}}=\frac{1-S_{O R}+K_{j} S_{O R}}{1-S_{O R}+K_{i} S_{O R}}$

Where $T_{j}$ and $T_{i}$ is the time of production of tracers $j$ and i respectively.

Rearranging Eq. 1 and solving for the residual oil saturation:

$S_{O R}=\frac{T_{j}-T_{i}}{T_{j}\left(1-K_{i}\right)+T_{i}\left(K_{j}-1\right)}$

Whenever tracer $i$ is a passive water tracer, $K_{i}=0$. Eq. 2 can be rewritten simply as:

$S_{O R}=\frac{T_{R}-T_{W}}{T_{R}+T_{W}(K-1)}$

Where $T_{R}$ is the residence time of the partitioning tracer, $T_{W}$ the residence time of the water tracer and $\mathrm{K}$ is simply the oil/water partition coefficient of the partitioning tracer.

Equation 3 is presently the most used form for determination of Sor. Cooke's original work, as well as the majority of PITTs performed, rely on the use of at least one passive water tracer. Dean's work demonstrated that it is also possible to use different partitioning tracers with different $\mathrm{K}$ values to measure $\mathrm{S}_{\mathrm{OR}}$. Fig. 1 illustrates the principle of a PITT.
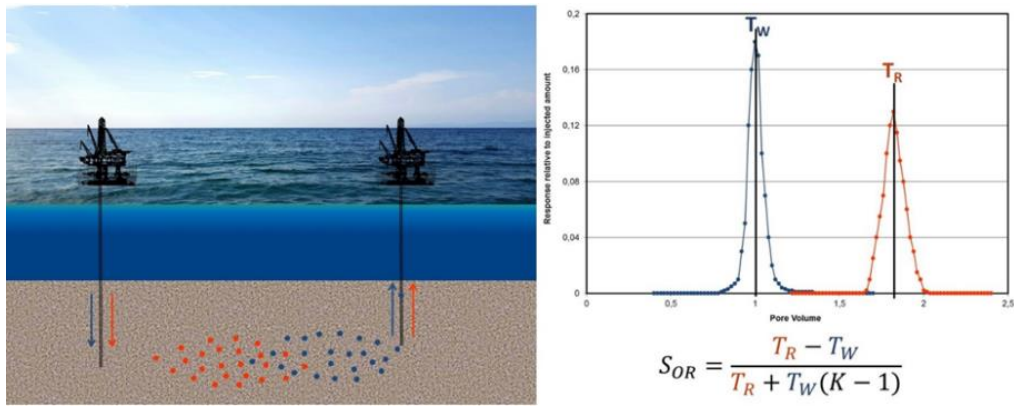

Figure 1. Illustration of the principle of a PITT. When traveling through a hydrocarbon bearing medium, the phase-partitioning tracer (orange dots) is delayed relatively to the passive water tracer (blue dots). Sor between injector/producer well pairs can be determined from the tracer production curves. 
In his original work (Cooke, 1971) used the detected breakthrough times as TR and TW. This drew criticism, as the time of breakthrough is often inaccurately determined due to dispersion, tracer detection limit, and streamline or layer distributions (Tang, 1995). Subsequent works to Cooke's patent also focused on the interpretation of production curves. (Tang, 1992; Tang and Harker, 1991) showed that higher accuracy on the determination of Sor can be achieved if the full production curves are used, or TR and TW are determined from using the same tracer recovery landmark different from the points of "breakthrough".

The PITT can be an important tool for reservoir characterisation, as the knowledge of $\mathrm{S}_{\mathrm{OR}}$ in the inter-well region is key information for efficient reservoir management and for the design and evaluation of IOR projects. However, very few PITTs in water flooded reservoirs have been reported since the introduction of test up to the 2000's. In 1990, (Wood et al., 1990) reported a PITT on the "Leduc Woodbend D-2A" field located in Alberta, Canada. The test used tritiated water and tritiated methanol as passive water tracers, and tritiated n-butanol as the oil/water partitioning tracer.

$S_{\mathrm{OR}}$ results were determined but significantly different recoveries were obtained for the partitioning tracers comparatively to the passive ones. Iodide as passive water tracer and phenol, and o-cresol as partitioning tracers were used in the "Chateaurenard field", located in the Paris basin, France (Causin et al., 1990). The tracer production curves exhibited several hard-to-explain anomalies and the determined Sor values using both partitioning tracers were so inconsistent that the validity of the test itself can be questioned (Tang, 1995). (Lichtenberger, 1991) presented and discussed the results of an inter-well tracer test performed on 3 different formations in the Ranger oilfield located in Texas, USA. Tritiated water, thiocyanate, ${ }^{57} \mathrm{Co},{ }^{58} \mathrm{Co}$, and ${ }^{60} \mathrm{Co}$ were used as passive tracers and isopropanol and tert-butyl alcohol as partitioning tracers. It is not clear from the publication in which chemical form the cobalt radionuclides were used (i.e. $\mathrm{Co}^{3+}$ or $\left.\mathrm{Co}(\mathrm{CN})_{6}{ }^{3-}\right)$. The recovery of the Co-isotopes were low, probably due to adsorption to reservoir rock. Both 
isopropanol, and tert-butyl alcohol were also found to adsorb somewhat to the reservoir rocks. Additionally, the methods used to analyse the partitioning tracers had very high detection limits $(\mathrm{mg} / \mathrm{L})$, which introduced additional errors when building the production curves. (Tang, 1992) reported on two PITTs performed 5 months apart on the "Judy Creek A" field in Alberta, Canada, between the same injector/producer well-pair. Tritiated water was used as passive tracer, while tritiated n-butanol and C-14 tagged isoamyl alcohol were used as phase-partitioning tracers. The tests produced good results, with similar recoveries of passive and partitioning tracers, and measured a decrease in $\mathrm{S}_{\mathrm{OR}}$ between tests in agreement with that estimated from production data. The 2 PITTs used exclusively radiolabelled tracers.

Several factors have contributed to the modest deployment of PITTs in the oilfield. It was just in the 1970's when new strategies (other than repressurizing the formation) to increase oil recovery started being considered, that the industry started considering tracer tests to measure Sor (Tayyib et al., 2019). (Deans, 1971) proposed and developed a method to determine $S_{O R}$ in the near-well region (some meters around the wellbore) known as the single-well chemical tracer test (SWCTT). In comparison to the PITT, the SWCTT samples a much smaller, thus less representative, volume of the reservoir. However, a SWCTT typically yields results within some days or weeks, while the time for a PITT ranges from weeks for small well distances to months and even years for large well distances.

Starting in the 1990's, environmental restrictions were put in place that severely limited or prohibited (in some countries) the use of radioactive tracers in oilfields (Serres-Piole et al., 2012). The implication was that the radiolabelled compounds that used to be applied in inter-well tracer tests with corresponding highly sensitive radioactive measuring techniques experienced a serious restriction. The quest was for replacing radiotracers with non-radioactive tracers. Thus, the limits of detection of the tracers became much higher (for example, for the alcohols previously used in PITTs, - in the low $\mathrm{mg} / \mathrm{L}$ range). In a PITT, the 
injected tracers typically are diluted by factors of $10^{9}-10^{12}$ (Dugstad et al., 2013) depending on the flooded volume between injection and production wells. Consequently, the amount of chemical tracers required became much larger. This makes the logistic for the PITT more complex, particularly in offshore reservoirs, and can significantly impact the cost of the test.

In the reported PITT field cases, it is not possible to exclude inappropriate behaviour of the compounds used from the abnormalities in the results. In fact, (Lichtenberger, 1991) reported that the partitioning tracers deployed adsorbed to the reservoir rock. Reversible adsorption can also cause a delay of the partitioning tracers relatively to the passive ones and would translate in the same type of response as partitioning to the oil phase on the production curves. While this has not been reported specifically for oil/water partitioning tracer candidates, (Bjørnstad et al., 1994) reported reversible adsorption of $\mathrm{I}^{-}$and $\mathrm{SO}_{4}{ }^{2-}$ when investigating new passive water tracers for chalk reservoirs and (Strand et al., 2006) developed a method for measuring wettability of chalk cores based on the reversible adsorption of $\mathrm{SO}_{4}{ }^{2-}$. Most of the molecules used in the early days of inter-well tracer tests in oil reservoirs were selected after their successful use in hydrogeology applications (Ahmad et al., 2003). However, the conditions encountered in an oil reservoir are significantly different from the ones in the near-surface subsoil. In summary, the limited use of PITT up to the 2000's was driven by the initial lack of interest from the oil industry, the increasing use of the faster SWCTT, a poor detection limits of the methods at hand to quantify non-radioactive tracers, and a selection of the partitioning compounds used which was based on a poor knowledge of their behaviour in an oil reservoir.

During the 2000's, the increase in the number of oilfields reaching maturity together with more serious investment on the development of EOR techniques, brought about also higher focus on reservoir characterisation tools such as the PITT. Beneficiating from the developments within analytical chemistry, both in instrumentation and sample preparation techniques, which made possible to analyse millions 
of different molecules in the ng/L range, (Viig et al., 2013) presented a successful PITT performed on the Lagrave field in France with undisclosed compounds specifically thought for such an application. The same compounds were again successfully used in the Bockstedt field in Germany (Hartvig et al., 2015), and on a carbonate land-based reservoir of undisclosed location (Sanni et al., 2018).

For the PITT to be used as a routine reservoir characterisation tool, it is critical that the accuracy of the test is ensured. The main objective of the present study is to introduce and apply a methodology for the selection and qualification of new oil/water partitioning tracers for inter-well applications. This methodology is based on a comprehensive approach based on the conditions encountered on the Norwegian continental shelf, consisting of a selection and qualification process that effectively minimizes the risk of unsuccessful field deployment of PITTs.

An oil/water partitioning tracer must obey a series of strict criteria to fit the scope of application of PITTs. Most of these criteria are common to all inter-well water tracers and should be applied when considering a new substance for testing as a PITT tracer. Studies focused on analysing the requirements for water tracers to be used in oil reservoirs [(Papers I and II) (referring to the "list of publications" in this thesis)] and the in-depth study by (Serres-Piole et al., 2012) show that many of the features a water-based tracer must exhibit are common regardless of its nature (passive or partitioning):

- The tracer must be thermally, chemically, and biologically stable for long periods of time.

- A passive water tracer must follow exclusively the water in which it is injected.

- The tracer must not interact with the reservoir rocks (i.e., not adsorb or change composition).

- A tracer must not influence the physical or chemical properties of the reservoir fluids.

- A tracer should not be naturally present, or be present in constant and very low levels, in the fluids of the reservoir. 
- A tracer must be quantifiable in very low concentrations to allow a low amount to be injected, but still keeping a sufficiently high accuracy of the production curves.

- A tracer must have an acceptable level of environmental toxicity.

- A tracer must be commercially available in sufficiently large quantities at an acceptable cost.

Most of these criteria are self-explanatory and reflect on the quality of the measurements that a PITT provide. They should also be considered within the process of selecting new chemical compounds for testing as oil/water partitioning tracer candidates (Papers I, II, and to a smaller extent, Papers III, IV and V). Fluorinated benzoic acids (FBAs) are widely used as passive water tracers in oil reservoirs in inter-well tracer tests (Asadi and Shook, 2010; Bjørnstad, 1991; Serres-Piole et al., 2011a; Serres-Piole et al., 2011b) and well-known for their thermal and chemical stability and absence of interaction with reservoir rocks. Figure 2 depicts the generic structure of FBAs.<smiles>[R]c1c([R])c([R])c(C(=O)O)c([R])c1[R]</smiles>

Figure 2. Generic structure of fluorinated benzoic acids.

In figure 2 at least one of the R1 - R5 groups is a fluorine atom. This type of structure, with interpolate doble bounds between the carbon atoms, allows for chemical resonance in the molecule. Resonance contributes to the stability of a compound, as double bonds can shift from carbon pair to carbon pair, preserving the integrity of the ring. Considering the typical reservoir conditions (temperature, redox 
potential) encountered in the oilfields on the Norwegian continental shelf, molecules with structures that suggest high stability should be the first choice when new reservoir tracers are being considered. However, even molecules with a high stability can be affected due to the chemical nature of their substituents. Different compounds investigated as partitioning tracer candidates exhibit very different thermal and chemical stabilities as well as interaction with reservoir rocks, even within the same chemical families (Paper III, IV and V). Many of the findings about some compounds deny the possibility of their be use as PITT tracers. Other findings open the possibility of using inter-well tracers to retrieve more information about the reservoir. These aspects are a central part of this thesis and included static thermal stability experiments and respective discussion.

For the tracer candidates that exhibit sufficient stability and passivity towards the reservoir rock materials, it becomes crucial to develop suitable highly sensitive analytical methods to characterize its presence and concentration in reservoir fluids (Paper VI). Modern analytical chemistry techniques and instruments allow for the analysis of millions of molecules with high precision, accuracy, and sensitivity. Limits of quantification (LOQ) in the $\mathrm{ng} / \mathrm{L}$ or low $\mu \mathrm{g} / \mathrm{L}$ range are central to ensure the feasibility of a planned PITT (direct determination of the amount of tracer it requires), and to ensure the accuracy of the analysis. The lower the LOQs for the tracers used, the more accurate are their production curves for the same amount of injected tracer.

In addition to the aforementioned requirements, an oil/water partitioning tracer must also meet two additional criteria. These relate specifically to the oil/water partition characteristics imposed on these tracers by the concept of the PITT.

- An oil/water partitioning tracer must exhibit constant and reversible partitioning with oil under constant experimental conditions (Shook et al., 2009). Reversibility of the partitioning is a crucial feature of the partitioning tracer. When irreversible dissolution of a given substance in the hydrocarbon phase (partial or total) is observed, then that substance 
is not an oil/water partitioning tracer and cannot be used as such in a PITT. The "constant" partition refers to independence from concentration and phase the substance is initially dissolved in. The extent of partitioning (the value of $\mathrm{K}$ ) will always be influenced by parameters of the system (in oil reservoirs: water ion strength, $\mathrm{pH}$, redox conditions, temperature, oil composition etc.).

- The value of $\mathrm{K}$ must not be too high or too low. When the K-value is to low, insufficient separation between the tracers can occur in production, either making calculation of $\mathrm{S}_{\mathrm{OR}}$ impossible from the production curves or introducing very large errors. If the K-value is too high, the feasibility of the test can be compromised due to the long time for the partitioning tracer to be produced (many months or even years). Values of $\mathrm{K}$ in the range $2-5$ are a good indication for a successful field test (Tang, 1992).

The determination of the $\mathrm{K}$-values is done in the laboratory prior to the field test, using black oil from the field to be tested combined with produced water from the same field, at the temperature conditions observed (Cooke, 1971; Tang, 1992, 1995; Wood et al., 1990). The determined K-value is assumed to be static during the test and is used to calculate Sor. As previously briefly mentioned, the partitioning of chemical compounds between a hydrocarbon and an aqueous phase is known to be influenced by salinity, temperature, composition of the hydrocarbon phase, and in some instances pH (Austine et al., 2015; Dearden and Bresnen, 1988; Leo et al., 1971; Salager et al., 2000; Stephan et al., 2018). A thorough characterisation of the variation of the $\mathrm{K}$-value of the partitioning tracer (Paper VII) as function of these parameters is critical for the accuracy of the PITT. The present approach delivers information to create a "toolbox" that can correct for varying temperatures, water ion compositions, and oil compositions in the path between injection/producer pairs.

Dynamic flooding properties of the oil/water partitioning tracer candidates must also be evaluated. In fact, dynamic flooding properties of any type of tracer candidate for a complex porous medium should be 
evaluated. Previous studies about this topic are scarce. One notable exception is the work by (Bjørnstad et al., 1994). The authors evaluated seven radio-labelled water tracer candidates for carbonate reservoirs using tritiated water (HTO) as the reference water tracer. Ion exclusion effects were observed for two of the candidates $\left(\mathrm{S}^{14} \mathrm{CN}^{-}\right.$and $\left.{ }^{36} \mathrm{Cl}^{-}\right)$, a more complicated behaviour explained by a combination of ion exclusion, sorption/desorption and precipitation/dissolution was observed for ${ }^{131} \mathrm{I}^{-}$and ${ }^{35} \mathrm{SO}_{4}{ }^{2-}$. The cation ${ }^{22} \mathrm{Na}^{+}$exhibited reversible adsorption, and $\mathrm{H}^{14} \mathrm{CO}^{3-}$ could not at all be used as tracer in the studied system due to the carbonate-carbon dioxide equilibrium. (Austine et al., 2015) investigated the use of tracers to characterise the effects of an alkaline polymer surfactant (ASP) flooding in laboratory-scale core flooding experiments. HTO was used as the reference water tracer, and several anionic molecules were also tested as passive water tracers. The partitioning tracers used were the same as reported by (Viig et al., 2013). The authors reported ion exclusion for passive anionic water tracers while Sor measurements based on the production curves of oil/water partitioning tracers and HTO yielded satisfactory results. These examples serve to illustrate the importance of dynamic experiments to evaluate the flooding properties of new tracer candidates prior to their use in the field. Deviations from an ideal flooding behaviour that might appear small on a laboratory scale, have the potential to significantly impact the information retrieved from a field test. Such deviations may be magnified due to the sheer scale (from $\mathrm{cm}$ in the laboratory to several hundred or thousand meters in a reservoir). However, such small deviations from ideality detected in lab experiments may also "drown" on the field scale due to dispersion of the fluid flow itself. The dynamic flooding properties of the PITT tracer candidates tested in the present work were evaluated in laboratory experiments using cores/columns prepared both to full water saturation and to SOR conditions.

Overall, the qualification of tracers prior to their use in the field is a process that has been somewhat neglected or kept under commercial secrecy. Thus, most of the published information about the use of tracers 
in the oilfield consists of the reporting of successful tests employing molecules which have been successfully used in earlier tests. In fact, (Tang, 1992) attributes the historical lack of widespread use of PITT also to a poor selection of the compounds used as PITT tracers.

The contents of the present study offer an integrated methodology to select and test oil/water partitioning molecules prior to field tests. This methodology combines a series of static and dynamic experiments further described in the "methods" section. All the findings from these experiments rely on our ability to identify and quantify the tracer candidates with high accuracy. Therefore, the present study has included the use of advanced instrumental analytical chemistry techniques. The techniques used were as follows:

- Gas chromatography coupled with flame ionisation detection (GC-FID).

- Gas chromatography coupled with mass spectrometry and tandem mass spectrometry (GC-MS/MS).

- Ultra-performance liquid chromatography coupled with ultraviolet detection (UPLC-UV).

- Ultra-performance liquid chromatography coupled with high resolution mass spectrometry (UPLC-HRMS).

- Liquid scintillation counting (for determination of beta particle radiation of radiotracers used in the laboratory qualification experiments).

By combining static and dynamic experiments, a wide range of data is produced that allows for a thorough understanding of the behaviour of the tracer candidate under a wide range of reservoir conditions, as well as to explore their features in other possible applications. For example, in Papers III and V two tracer candidates that were found to thermally degraded are presented. The degradation fits a first order kinetic model, thus opening the possibility of using these compounds to retrieve thermal information from the flooded sections of oil reservoirs. 
Another example is the particular interaction (probably sorption) between molecules with methoxy groups as substituents and the clay (kaolinite) used in the experiments, described in Papers IV and V.

These are just two examples of curious findings in addition to the focus of the study: the pursuit of new oil/water partitioning tracers. In Paper VI we introduce a sample preparation methodology, never used before, to identify and quantify the stable tracer candidates in real produced waters from the Norwegian continental shelf. This approach led to detection of the PITT tracer candidates at concentrations in the low $\mathrm{ng} / \mathrm{L}$ range. Phase equilibria and influence of ion strength, hydrocarbon composition and temperature, are evaluated in Paper VII. Thus, the present study is a mixture of basic and applied science with results which should have high interest for reservoir engineers, petrochemists, tracer specialists with both operator and service companies, reservoir modelers, research scientists working on the development of tracers or with applications of tracers, chemists, and analytical chemists. 


\section{Materials and methods}

\subsection{Selection of the partitioning tracer candidates}

The selection of the oil/water partitioning tracer candidates was the first step of the present work. Little information is available in the literature about the development of PITT tracers. Thus, the selection was based on four primary required and/or desired features of the molecules to be tested:

- $\quad$ Solubility in both phases: the partitioning tracer must be soluble in both hydrocarbon and aqueous phases (Cooke, 1971) and is to be deployed and followed in the injected and produced water, respectively. Compounds which form ions in aqueous solution will not be a partitioning tracer since ions are not soluble in hydrocarbon phases. Thus, the oil/water partitioning tracer candidates should be compounds that are water soluble due to dipole interactions and/or hydrogen bridges. An exception to this requirement is that of compounds which may ionise to a larger or smaller extent as function of $\mathrm{pH}$, essentially weak acids, or weak bases. Due to the equilibrium established between the ionic form, and the distribution of the non-ionic form between two phases they can in theory be used (in combination with other partitioning compounds) to retrieve information about $\mathrm{pH}$ conditions along different segments of the reservoir. This concept is further explored in Paper V. Additionally, different substituents on the same base molecule can have dramatic effects on its affinity towards hydrocarbon phases (lipophilicity) or aqueous phases (hydrophilicity). The higher the lipophilicity of a molecule the more it dissolves in the hydrocarbon phase (and becomes less soluble in water). Naturally, the inverse is also true.

- The "predicted" K-value: experimental and predicted values of the 1-octanol/water partition coefficient are readily available for millions of chemical compounds in chemistry databases. Similar to K, the 1octanol/water partition coefficient $(\mathrm{P})$ is defined as the equilibrium concentration distribution of a given chemical species between pure 
octanol and deionised water. It is commonly expressed in a logarithmic scale as $\log \mathrm{P} . \log \mathrm{P}$ was early identified as an indicator of the potential biological activity and tendency for bioaccumulation of chemical substances (Sangster, 1989), since the 1-octanol/water system is found to mimic the intracellular fluid/lipid membranes system of living systems (Schwarzenbach et al., 2002). This is the primary reason why LogP has been determined or estimated for so many compounds. The difference in polarity between octanol and water is typically bigger than between oil (crude oils contain many polar compounds, such as organic acids, bases, and oxygenated hydrocarbons) and the formation or injected water. Thus, for a given temperature, $\mathrm{P}$ should be higher than $\mathrm{K}$, however it will provide a good indication about the range of $\mathrm{K}$ values expected from a given molecule.

- $\quad$ Chemical structure: molecules without functional groups which can be easily oxidised or reduced but with a structure that allows for chemical resonance are more likely to possess the required stability. The presence of functional groups will, however, often be crucial to ensure a good water solubility. For example, alcohols are an obvious chemical "family" to consider when selecting new PITT tracers, as they typically do not ionise (to a significant degree) in aqueous solution. However, when their functional group $(\mathrm{OH})$ is directly bonded to the main chain, they can be more easily oxidised when the minimum redox potential and oxygen are present, even in molecules with aromatic, cyclic or heterocyclic structures that allow for resonance. (Tang, 2003) evaluated the possibility of using phenol leached from bitumen to trace spills of this material. Loss of phenol due to adsorption on sand and possible oxidation was observed in near-surface aquifers. Benzyl alcohol is characterised by a $\mathrm{CH} 2$-group between the aromatic benzene ring and the alcohol group. This branch makes the alcohol less reactive as it "buffers" the effect of the $\pi$-electrons on the functional group. This will, in principle, increase the stability of the molecule. Molecules successfully used in the past as tracers in inter-well applications also provide guidance in the selection of new ones. Similar structures are, in 
principle, more likely to exhibit the thermal stability required, and thus should be observed in the selection of new candidates.

- $\quad$ Analysability: the ability to identify and quantify the tracers in real production waters at least in the low $\mu \mathrm{g} / \mathrm{L}$ range, is a key characteristic for a PITT. Low quantification limits mean more accurate production curves and less amount of tracer injected. The latter leads to smaller environmental impact of the operation and lower costs (both from the purchase of chemicals and their handling). It is therefore desirable that the tracer candidates can be analysed by several techniques, so that the method development can be as flexible as possible to overcome possible matrix effects from field samples.

- Commercial availability and price: for practical purposes, the molecules used as tracers in inter-well applications should be available commercially in sufficient amount to an affordable price or be easily synthesized in the laboratory.

Using the "Chemistry WebBook" from the National Institute of Standards and Technology (NIST) of the U.S. Department of Commerce, and based on the previous presented considerations, a total of 34 molecules were initially identified for further evaluation. Of these, 16 molecules from 4 chemical families (pyrazines, benzyl alcohols, pyridines, and linear diols) were selected for testing within the frame of the present work. Table 1 presents their identity, acronyms, and some relevant physico-chemical properties. 
Materials and methods

Table 1 - Identification and relevant properties of the molecules selected as new oil/water partitioning tracer candidates

\begin{tabular}{|c|c|c|c|c|c|c|}
\hline Compound & Acronym & $\begin{array}{l}\text { Melting } \\
\text { point }\left({ }^{\circ} \mathrm{C}\right)\end{array}$ & $\begin{array}{l}\text { Boiling } \\
\text { point }\left({ }^{\circ} \mathrm{C}\right)\end{array}$ & $\begin{array}{l}\text { Vapour pressure } \\
\text { at } 25^{\circ} \mathrm{C} \text { (mTorr) }\end{array}$ & $\log \mathrm{P}$ & $\mathrm{pKa}$ \\
\hline 2,3-Dimethylpyrazine & 23MPRZ & -12 & 156 & $2,74 \times 10^{3}$ & 0,54 & $1,62 *$ \\
\hline 2,6-Dimethylpyrazine & 26MPRZ & 37 & 154 & $2,55 \times 10^{3}$ & 0,55 & $1,55 *$ \\
\hline 2-Methoxypyrazine & 2METPRZ & 32 & 169 & $1,14 \times 10^{3}$ & 0,73 & $1,51 *$ \\
\hline 2-Fluoropyrazine & 2FPRZ & -6 & 110 & $1,05 \times 10^{3}$ & 0,29 & $1,74 *$ \\
\hline 2-Chloropyrazine & 2CIPRZ & 20 & 154 & $1,82 \times 10^{3}$ & 0,64 & $1,58 *$ \\
\hline 4-Hydroxybenzyl alcohol & 4HYBZOH & 114 & 251 & 0,142 & 0,25 & 9,82 \\
\hline 4-Chlorobenzyl alcohol & $4 \mathrm{ClBZOH}$ & 71 & 232 & 0,268 & 0,82 & 13,9 \\
\hline 2,6-Dichlorobenzyl alcohol & $26 \mathrm{ClBZOH}$ & 98 & 268 & 0,254 & 1,07 & 13,5 \\
\hline 4-Methoxybenzyl alcohol & 4METBZOH & 23 & 259 & 0,082 & 0,71 & 13,6 \\
\hline 3,4-Dimethoxybenzyl alcohol & 34METBZOH & 22 & 297 & 0,0696 & 0,62 & 13,3 \\
\hline Pyridine & PYR & -42 & 115 & $19,3 \times 10^{3}$ & 0,65 & $5,25 *$ \\
\hline 4-Methoxypyridine & 4METPYR & 4 & 191 & $17,6 \times 10^{3}$ & 0,71 & $6,58 *$ \\
\hline 3-Hydroxypyridine & 3HYPYR & 126 & 329 & $7,44 \times 10^{3}$ & 0,64 & $4,79 * / 8,75$ \\
\hline 2-Hydroxy-6-methylpyridine & 2HY6MPYR & 159 & 275 & $8,21 \times 10^{3}$ & 0,34 & $4,94 * / 9,27$ \\
\hline 1,2-Hexanediol & 12HEXDIOL & 2 & 223 & 0,422 & 0,69 & 13,7 \\
\hline 1,6-Hexanediol & 16HEXDIOL & 41 & 248 & 0,457 & 0,76 & 13,7 \\
\hline
\end{tabular}

* Value for the mono-protonated species

\subsection{Materials}

The oil/water partitioning tracer candidates identified in section 2.1., were purchased from speciality chemical companies in their highest purity grade commercially available. Rock materials and consolidated cores were also a central part of the experiments performed in the present 
study. Next, a short description of the most relevant "materials" employed is presented.

\subsubsection{Pyrazines}

The molecular structures of the tracer candidates in the pyrazine family are given in Figure 3.

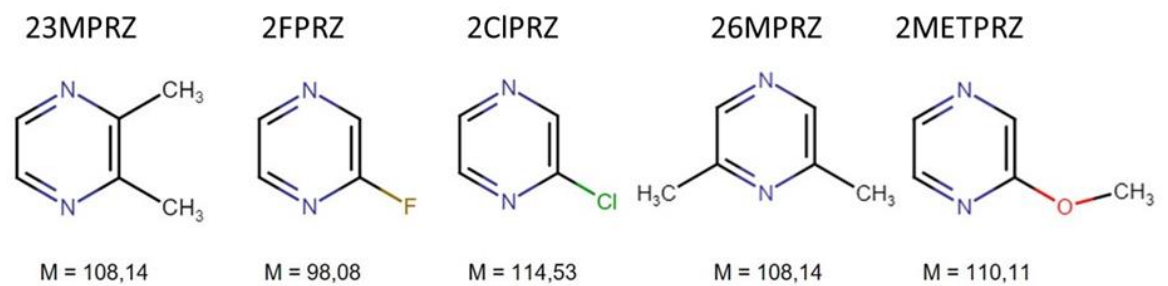

Figure 3. Structure and molecular mass (M) of the pyrazines selected as oil/water partitioning tracer candidates.

Pyrazines are monocyclic aromatic rings with two carbon atoms substituted by nitrogen in the para position. They are characterised by a strong pungent smell due to their relatively high volatility and very low olfactory detection threshold. Pyrazines are formed by the reaction between amino acids, peptides, or proteins and carbonyl compounds described by the non-enzymatic Maillard reaction (Müller and Rappert, 2010; Yu et al., 2017). The highest yields of production of these chemicals are obtained at temperatures between $120^{\circ} \mathrm{C}-150{ }^{\circ} \mathrm{C}$ (Müller and Rappert, 2010) and in alkaline conditions (Jousse et al., 2002; Yu and Zhang, 2010). Pyrazines can be formed by thermally based processing of food products (roasting, grilling, baking) and are therefore constituents of the flavour and scent of many human feedstuff. Alkylpyrazines (such as 23MPRZ and 26MPRZ), and methoxypyrazines (such as 2METPRZ) have been identified as major scent and flavour contributes in coffee (Blank et al., 1992), corn products (Buttery et al., 1994), roasted beef (Cerny and Grosch, 1994), or roasted nuts (Alasalvar et al., 2003), for example. Commercially available pyrazines are obtained from industrial chemical synthesis with a yearly production of 
a few tones used almost entirely by the food industry (Müller and Rappert, 2010). Most alkyl and methoxypyrazines (also the ones included in the present study) have long been deemed "safe" for use as food additives (Adams et al., 2002) and small amounts of these compounds produce the desired output, due to their strong aromatic properties. Both 2FPRZ and 2ClPRZ are exclusively anthropogenic and have very limited use. The only reported use of $2 \mathrm{FPRZ}$ is as monomer of building blocks for production of self-assembling coordinated polymers (Takahashi et al., 2014; Valverde-Muñoz et al., 2016), while 2ClPRZ is exclusively used as precursor of active principles in the pharmaceutical industry (Hirsh et al., 2008; Mannam et al., 2019). Pyrazines are, in general, safe chemicals both for humans and for ecosystems whose occurrence, production and use suggests little likelihood of presence in the oilfield. None of the pyrazines used in the present study was, to the best of the author's knowledge, ever reported in any of the fluids present in the oilfield or identified during the present study in production waters from the Norwegian continental shelf (Paper VI).

\subsubsection{Benzyl alcohols}

The molecular structures of the tracer candidates in the benzyl alcohol family are given in Figure 4.

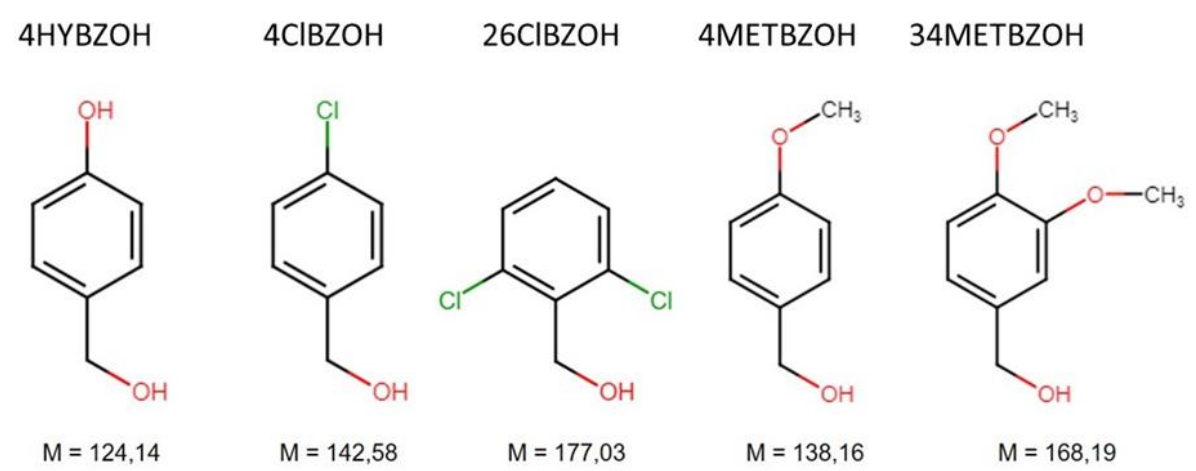

Figure 4. Structure and molecular mass (M) of the benzyl alcohols selected as oil/water partitioning tracer candidates. 
4HYBZOH is a benzyl alcohol with one hydrogen substituted by a hydroxy group in the p-position, making the molecule simultaneously a phenol. 4HYBZOH is a weak acid with a pKa of 9,82, thus most likely protonated in the typical range of $\mathrm{pH}$ encountered in oilfields of the Norwegian Continental Shelf. The presence of the hydroxy group also increases the hydrophilicity of the molecule in comparison to its base structure (benzyl alcohol). 4HYBZOH is a colourless liquid with a mild and pleasant scent with authorised used as food additive in restricted amounts by the "regulation (EU) No 872/2012 of 1 October 2012". It has been identified in small amounts in Arcangelisia gusanlung (a small shrub from China) and has little industrial use beyond the food industry, with specialty applications in the pharmaceutical (Luo et al., 2017) and materials (Tao et al., 2017) industries. Chlorinated benzyl alcohols have never, to the best of the author's knowledge, been reported to occur naturally, thus are exclusively anthropogenic. $4 \mathrm{ClBZOH}$ and $26 \mathrm{ClBZOH}$ are white powders, slightly soluble in water without any acidic or basic properties. They have small industrial use as precursors for production of aromatic aldehydes, as model compounds to study the oxidation of substituted benzyl alcohols, and to evaluate the performance of selective catalysed processes aiming for production of aromatic aldehydes (Esteruelas et al., 2011; Higashimoto et al., 2009; Scandura et al., 2016). Specifically, 4CIBZOH has been used to assess catalysts for the FriedelCrafts alkylation of aromatic hydrocarbons (Mantri et al., 2005). 4METBZOH and 3,4METBZOH are benzyl alcohols with one and two hydrogens, respectively, substituted by methoxy groups. They have a neutral character in water solution and, as consequence of the methoxy groups, are more hydrophilic than their base structure. Methoxybenzyl alcohols occur naturally in trace amounts in specific species of fungi and are produced industrially. Like the chlorinated benzyl alcohols in the present study, 4METBZOH and 3,4METBZOH have limited industrial applications, and are primarily used as precursors for production of aromatic aldehydes (Higashimoto et al., 2009; Morad et al., 2017; Scandura et al., 2016). To the best of the author's knowledge, none of 
the benzyl alcohols contained in the present study has ever been reported as constituent of any oil reservoir fluids.

\subsubsection{Pyridines}

The molecular structures of the tracer candidates in the pyridine family are given in Figure 5.
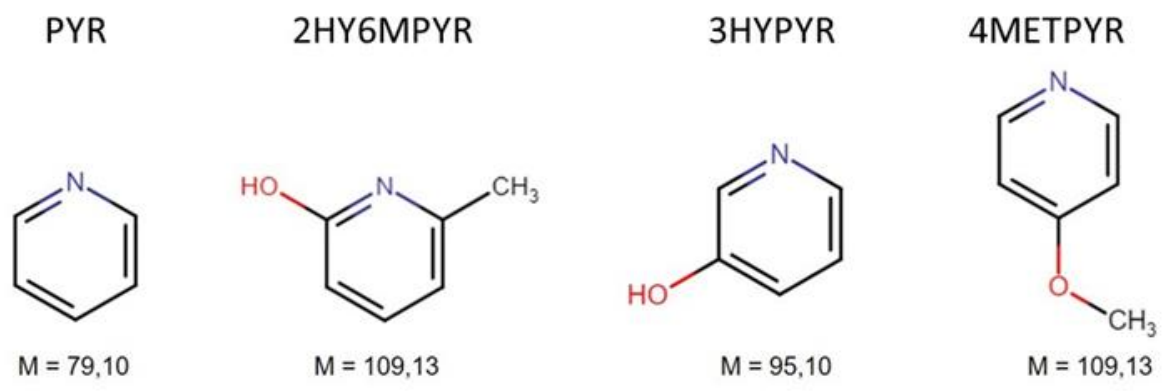

Figure 5. Structure and molecular mass (M) of the pyridines selected as oil/water partitioning tracer candidates.

Pyridine is a heterocyclic aromatic ring with one carbon atom substituted by a nitrogen atom. Pyridine, and most of its substituted species, are characterised by a distinct highly unpleasant smell, weak basic properties, and high-water solubility. Polycyclic pyridine derivatives (primarily) and pyridine have been reported as constituents of crude oils (Bae et al., 2010; King et al., 1973). They make up for $20 \%-26 \%$ of the total nitrogen content in all crude oils (King et al., 1973; Prado et al., 2017) which is usually $\leq 1 \%$ (wt.) and $<0.25 \%$ (wt.) in about $90 \%$ of them (Prado et al., 2017). The substituted pyridines included in the present study have never, to best of the author's knowledge, been reported as constituents of any reservoir fluid. However, it is also likely that these compounds are also present in trace amounts in hydrocarbons originated in oil reservoirs. Pyridine is frequently used in the chemical industry, primarily in the production of pesticides, as solvent, in the production of dyes, explosives, pharmaceuticals, and as ligand in specialty coordination chemistry synthesis (Ahsan et al., 2018; Liu et al., 
1998; Shen et al., 2015). 4METPYR has been reported as ligand in coordination chemistry (Mautner et al., 2018) and as precursor for the synthesis of active principles by the pharmaceutical industry (Yamada et al., 2017). 3HYPYR has been investigated as a monomer for production of polymers for $\mathrm{CO}_{2}$ capture (Gutiérrez et al., 2011), but its primary use is as precursor for pharmaceutical active principles (Kaviani and Izadyar, 2018). No significant large-scale use of 3HYPYR has been reported, however it is used on a laboratory scale as ligand in the preparation of special metal complexes. Nowadays, pyridines are exclusively produced industrially.

The inclusion of this class of chemicals in the present study was done with two primary goals (if these compounds actually qualified in the experiments performed): i) PITTs are designed for mature oilfields and it was thought that likely, one or more of the compounds in the present study could be present in low concentration in the production waters. If this were verified, tests to evaluate if the unequivocal identification and quantification of an injected tracer pulse could be performed relatively to the background "noise". ii) explore the $\mathrm{pH}$ dependency of the partitioning of the compounds of this class of chemicals relatively to oil/water partitioning tracers with partitioning independent from $\mathrm{pH}$, to try to obtain $\mathrm{pH}$ data from the inter-well region. Naturally, these two objectives were conditioned by the performance of the investigated pyridines in the experiments and to further characterisation during a sufficiently long period of time of the production water from the oilfield considered for a PITT test. Additionally, they would always be used in combination with other phase-partitioning tracers that do not exhibit any of the features $(\mathrm{pH}$ dependant partitioning and upfront presence in the production water) under evaluation for pyridines.

\subsubsection{Aliphatic diols}

The molecular structures of the tracer candidates in the family of aliphatic diols are given in Figure 6. 
12HEXDIOL<smiles>CCCCC(O)CO</smiles>

$M=118,17$
16HEXDIOL<smiles>OCCCCCCO</smiles>

$M=118,17$

Figure 6. Structure and molecular mass (M) of the aliphatic diols selected as oil/water partitioning tracer candidates.

Simple linear or branched isotopically labelled alcohols (butanol, isopropanol, tert-butyl alcohol and isoamyl alcohol) were used in the past as oil/water partitioning tracers in PITTs (Lichtenberger, 1991; Tang, 1992; Wood et al., 1990). The inclusion of 12HEXDIOL and 16HEXDIOL in the present study aimed at evaluating the possibility of using longer chain linear polyols. This would be an excellent possibility to "tune" the lipophilic/hydrophilic properties of the tracers, since the Kvalue could be "adjusted" by the number and position of the hydroxyl groups. If the two diols yielded promising results, further investigations into polyols would be advisable. For example, evaluating how they could be "tagged" to be more easily analysable by using radioactive labels.

12HEXDIOL and 16HEXDIOL are industrially synthesised through hydrogenation and/or hydration of adipic acid or its esters (Werle et al., 2008). 12HEXDIOL is primarily used in personal and skincare products as an emollient, humectant, and moisture-boosting agent. 16HEXDIOL is used for industrial production of polyesters and polyurethanes (Nasr et al., 2020; Werle et al., 2008). These compounds have never, to the best of the author's knowledge, been described as constituents of any fluids present in the oilfield.

\subsubsection{Rock substrate - Berea sandstone}

Outcrops of consolidated sandstone from the Norwegian continental shelf are difficult to obtain due to the high cost of extraction of the core material. Thus, laboratory experiments are often performed with 
consolidated sandstones from other origins. Many quarries of sandstone with similar properties to the formations encountered on the Norwegian continental shelf are available throughout the world. Naturally, the rock material originated in these quarries will have somewhat different chemical and mineralogical compositions as well as mechanical properties. However, for the fundamental parameters of the present study (chemical interactions of molecules in trace amounts), the outcrops from different quarries will perform as an excellent "model" material. One of the most used outcrops in laboratory experiments for or by the oil industry is Berea sandstone.

Berea sandstone (sometimes referred to as Berea grit) is a consolidated sandstone formation present throughout the U.S. states of Michigan, Ohio, Pennsylvania, West Virginia, and Kentucky. It was formed during the late Devonian period (about 370 million years ago) and is known to bear significant oil \& gas reserves (Menéndez et al., 1996). Berea sandstone consists primarily of sub-angular grains of quartz and potassium feldspar. Quartzite rock fragments and kaolinite clay aggregates and various accessory minerals, such as sodium feldspar, apatite, rutile and zircon, are also present (Menéndez et al., 1996; Zhang et al., 1990). The hydrocarbon-free cores of Berea sandstone used in the present study were purchased from Berea Sandstone ${ }^{\mathrm{TM}}$ Petroleum Cores (Ohio, USA). Their chemical composition, according to the supplier, is as follows: $93.13 \%$ silica $\left(\mathrm{SiO}_{2}\right), 3.86 \%$ alumina $\left(\mathrm{Al}_{2} \mathrm{O}_{3}\right), 0.11 \%$ ferric oxide $\left(\mathrm{Fe}_{2} \mathrm{O}_{3}\right), 0.54 \%$ ferrous oxide $(\mathrm{FeO}), 0.25 \%$ magnesium oxide $(\mathrm{MgO})$, and $0.10 \%$ calcium oxide $(\mathrm{CaO})$. Also, according to the supplier, the water permeability of the cores is $4,7 \times 10^{-14} \mathrm{~m}^{2}$ at an effective pressure of $3 \mathrm{MPa}$, and the porosity ranges between $21 \%-27 \%$.

\subsubsection{Rock substrate - Limestone}

Limestone is a sedimentary carbonate rock composed primarily of calcite and aragonite. Like most sedimentary rocks, it has a granular structure mainly as result of skeletal fragments of marine organisms such as coral or foraminifera (Lucia, 1995). In comparison to chalk it presents a more 
complex chemistry with higher content of minerals containing magnesium, iron, aluminium, and silica (Morse and Arvidson, 2002). The limestone used in the present study was purchased in powder form $(125 \mu \mathrm{m}-250 \mu \mathrm{m})$ from Sigma-Aldrich (Sigma-Aldrich Norway AS, 0252 Oslo). It intends to be a "model" rock to evaluate possible chemical interactions between a carbonate-based rock and the PITT tracer candidates in static experiments.

\subsubsection{Rock substrate - Kaolinite}

Kaolinite is a type of clay that has been reported as one of the most frequent clays occurring in hydrocarbon bearing sedimentary basins (Jiang, 2012; Menéndez et al., 1996). Kaolinite exhibits a layered structure, like sheets of paper laid over each other, alternating one tetrahedral sheet of silica bonded through oxygen to one octahedral sheet of alumina (Babu Valapa et al., 2017). Kaolinite exhibits extensive adsorbent characteristics both for inorganic and organic compounds (Bhattacharyya and Gupta, 2008; Yu et al., 2013), as well as catalytic activity for a wide range of reactions (Belver et al., 2002; Li et al., 2019; Vorlicek and Helz, 2002). Even though clays are present in small amounts in the oilfields (when compared to the major rock constituents) they can have significant interactions with the chemicals used as tracers. Therefore, kaolinite has been included as "model" clay in the present study. Powder kaolinite (natural aluminium silicate $125 \mu \mathrm{m}-250 \mu \mathrm{m}$ with linear formula $\mathrm{Al}_{2} \mathrm{O}_{3} \cdot 2 \mathrm{SiO}_{2} \cdot 2 \mathrm{H} 2 \mathrm{O}$ ) was purchased Sigma-Aldrich (Sigma-Aldrich Norway AS, 0252 Oslo).

\subsubsection{Rock substrate - Stevns Klint chalk (Sigerslev member)}

Stevns Klint chalk, specifically the Sigerslev variant used in the present study, is a sedimentary carbonate rock formed in the later part of the Maastrichtian period (Surlyk et al., 2010) composed of about 99\% carbonate minerals and trace amounts of quartz, clay, feldspar and apatite (Hjuler and Fabricius, 2009). Its typical porosity is $42 \%-50 \%$ and permeability $1-2 \mathrm{mD}$ (Hedegaard and Graue, 2011; Madland et al., 
2011). Stevns Klint chalk was used in the present study as "model" carbonate rock for evaluating the dynamic flooding properties of the PITT tracer candidates. Cores of this material, with the required dimensions, were cropped from a block of chalk.

\subsection{Methods}

A series of experiments under relevant conditions were devised to evaluate the selected partitioning tracer candidates. The final step of any qualification process for tracers should include at least one field pilot. This was impossible to perform during the three available years for the present study. The sequence of experiments for a complete tracer qualification process may be divided into five major groups. This sequence and its respective status for the candidates in the present study, are summarised in Table 2. Section 2.31. includes quality control results which are not published elsewhere. These results are not relevant for the main conclusions of the present study, however they are relevant to demonstrate the reliability of the methodology adopted. 
Table 2 - Steps and status of the methodology developed for qualification of new oil/water partitioning tracers for inter-well application.

\begin{tabular}{lll}
\hline \multicolumn{1}{c}{ Step } & \multicolumn{1}{c}{ Goal } & \multicolumn{1}{c}{ Status } \\
\hline $\begin{array}{l}\text { Development } \\
\text { of analytical } \\
\text { methods }\end{array}$ & $\begin{array}{l}\text { Obtain suitable, reliable, and efficient } \\
\text { analytical methods for identification and } \\
\text { quantification of the compounds in laboratory } \\
\text { and field samples. }\end{array}$ & Performed \\
$\begin{array}{l}\text { Static stability } \\
\text { experiments }\end{array}$ & $\begin{array}{l}\text { Evaluate long-term thermal stability and } \\
\text { characterise interactions with typical } \\
\text { reservoir rock constituents. }\end{array}$ & Performed \\
$\begin{array}{l}\text { Characterisati } \\
\text { on of the K- } \\
\text { value }\end{array}$ & $\begin{array}{l}\text { Understanding variations of the K-value as } \\
\text { function of relevant parameters to improve } \\
\text { accuracy in the determination of SoR values. }\end{array}$ & Performed \\
$\begin{array}{l}\text { Dynamic } \\
\text { flooding } \\
\text { experiments }\end{array}$ & $\begin{array}{l}\text { Evaluate the dynamic behaviour of the stable } \\
\text { candidates in consolidated rock materials at } \\
\text { water and oil saturation conditions. }\end{array}$ & Performed \\
Field test & $\begin{array}{l}\text { Deploy, on a well-characterised oilfield, the } \\
\text { tracer candidates that were qualified by the } \\
\text { previous steps for final verification. }\end{array}$ & Not performed \\
\hline
\end{tabular}

\subsubsection{Analytical methods for laboratory samples - I}

All experiments planned rely on the ability to identify and quantify the PITT tracer candidates in aqueous (and sometimes hydrocarbon) solutions. Thus, the development of suitable analytical methods was the first step of the experimental work of the present study. Liquid-phase chromatography with UV detection is an obvious choice for analysis of the tracer candidates in an aqueous phase. This was the main method used during the static stability experiments. Except for 12HEXDIOL and 16HEXDIOL, all other 14 PITT tracer candidates are characterised by a core cyclic molecular structure with resonances in the UV/visible wavelength region when excited by UV light. The conjugated $\pi$-bonds that characterise aromatic rings will absorb UV radiation with a wavelength around $210 \mathrm{~nm}$, depending exactly on the number and type of atoms that constitute the molecules. This also allows some qualitative 
information to be obtained about the analytes, since single bonds (present in virtually every organic molecule) absorb radiation at much lower wavelengths. Information about the UV absorption bands of the target analytes are readily available in several databases. After analysis of this data, $222 \mathrm{~nm}$ and $254 \mathrm{~nm}$ were selected as the wavelengths that would provide the best compromise between maximising absorption signals and detecting all compounds.

Reverse phase (RP) chromatography relies on lipophilic interactions with a solid support to obtain separation of the analytes. In principle, the more lipophilic a compound is, the stronger will the retention be in such a chromatographic system. The mobile phase for elution of the compounds can be adjusted as needed with organic and aqueous solvents to transport all the adsorbed/retained analytes to the detector within an acceptable time. Thus, UPLC-UV was the approach used for the analysis of all tracer candidates in this study, except 12HEXDIOL and 16HEXDIOL.

A description of the instrument used, and method developed is presented hereafter:

An "Agilent Technologies 1290 Infinity II" UPLC system (Agilent Technologies, Santa Clara, California, USA) equipped with a Waters "Acquity UPLC BEH" reversed-phase C18 $1.7 \mu \mathrm{m}$ packed column (100 mm X 2,1 mm) (Waters Corporation, Milford, Massachusetts, USA) was used. A possible transfer of the method developed in this system to a system coupled with MS detection was considered. Thus, all eluents used in the chromatographic system were comprised of volatile chemicals. Three eluents were used: an aqueous phase of $5 \mathrm{mM}$ ammonium formate buffer (A), methanol (B), and acetonitrile (C), all UPLC grade.

The chromatographic conditions which provided the best separation of the analytes were as follows:

- total flow of $0,5 \mathrm{~mL} / \mathrm{min}$ with gradient elution.

- Initially $98 \%$ of A and $1 \%$ of B. 
- The amount of B increased to $20 \%$ after 4 minutes and was kept at this value for 1.5 minutes.

- Fraction of B was then increased until $70 \%$ at minute 8 and kept at this value during $0.5 \mathrm{~min}$.

- Gradient conditions were then reset to the initial ones at minute 9 and held for 1 minute.

- The amount of eluent $\mathrm{C}$ was constant at $1 \%$ during the 10 minutes chromatographic run.

- The column was kept at $60^{\circ} \mathrm{C}$ during the whole run and $10 \mu \mathrm{L}$ of sample were injected after filtration through $0,45 \mu \mathrm{m}$ PTFE filters.

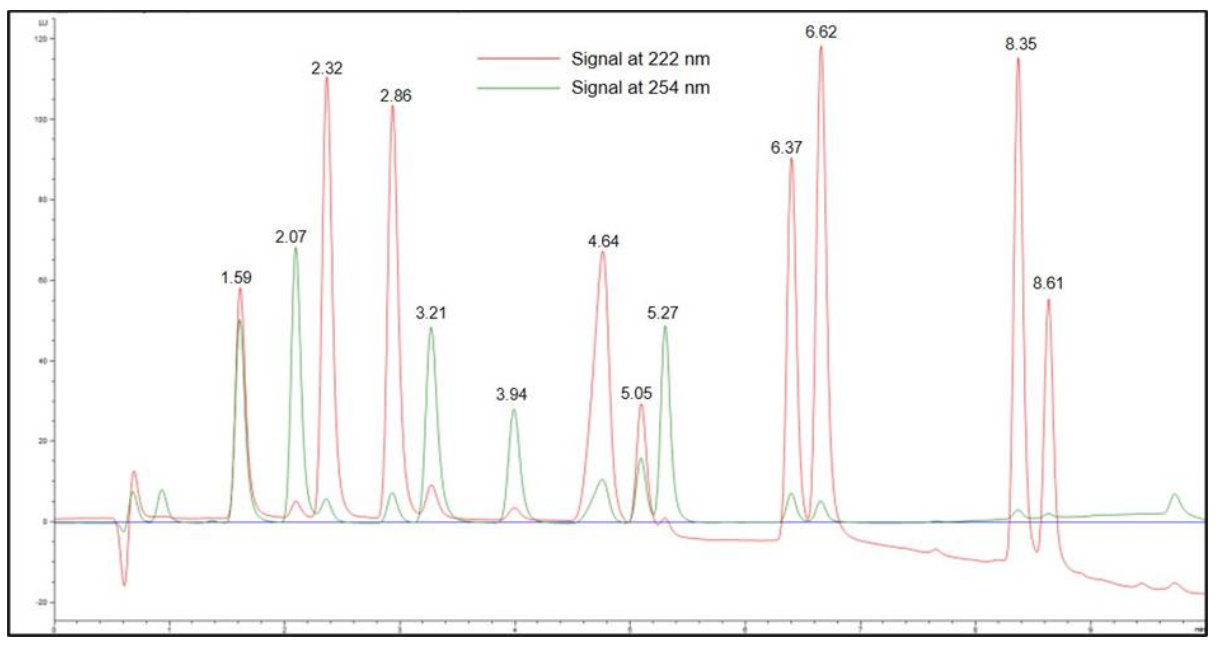

Figure 7. Example UPLC-UV chromatogram of a mixture of the 13 tracers analysable by the method developed at a concentration of $10 \mathrm{mg} / \mathrm{L}$.

Separation of 23MPRZ and 26MPRZ proved impossible under the analytical conditions encountered. These two compounds have very similar lipophilicities and acid-base characteristics, thus they tend to coelute. For this reason, subsequent modifications (after the static stability experiments) were performed to analyse these two compounds together. Before this, 26MPRZ was tested individually. 
Figure 7 depicts an example chromatogram of a sample with a mix of the tracer candidates at a concentration of $10 \mathrm{mg} / \mathrm{L}$, obtained at the optimised conditions of the method developed. To identify the peaks, see retention times shown on the top of the peaks and compare with data in Table 3. The UPLC-UV method developed was intended for a fast and reliable analysis of the large number of samples that were going to be produced by the subsequent laboratory experiments. Thus, it was not exhaustively validated in accordance with analytical chemistry standards (Eurachem guidelines for validation of analytical methods). Limits of quantification (LOQ) were imposed and verified but not determined experimentally. These are lower than the presented, however the range of linearity of the method was suitable for the intended purposes. The reason for this approach is that the direct transfer of this method to real field samples would not be feasible: i) to much UV noise is likely expected from field samples, due to the significant amount of dissolved hydrocarbons in the production waters (not enough selectivity); ii) the desired LOQs for field samples are in the low $\mu \mathrm{g} / \mathrm{L}$, ideally in the $\mathrm{ng} / \mathrm{L}$ range. To achieve this low quantification limits, different types of detection other than UV are necessary. The method was calibrated in a concentration range between 0,5 and $20 \mathrm{mg} / \mathrm{L}$. The linearity, inter $(\mathrm{N}=5)$ and intraday $(\mathrm{N}=7)$ precision, as well as the recovery were evaluated. The recovery was calculated by spiking a known a portion of a sample with a known amount of the chemical compound and determining its concentration and that of the unspiked portion. From the difference in concentrations determined, recovery was calculated. The precision and recovery data were obtained at three different concentration levels. Table 3 presents the chromatographic retention times $\left(t_{R}\right)$, linear range, and precision for each of the 14 tracer candidates analysed by the RP UPLC-UV method. Data about recovery, is presented in Table 5. 
Materials and methods

Table 3 - Retention time (tR), linear range, LOQ, intra-day and inter-day precision for each of the PITT tracer candidates analysed by the UPLC-UV based method.

\begin{tabular}{|c|c|c|c|c|c|c|c|c|c|}
\hline \multirow{3}{*}{ Compounds } & \multirow{3}{*}{$t_{R}(\min )$} & & & \multicolumn{6}{|c|}{ Precision (\% RSD) } \\
\hline & & \multicolumn{2}{|c|}{ Linearity } & \multicolumn{3}{|c|}{ Intra-day precision } & \multicolumn{3}{|c|}{ Inter-day precision } \\
\hline & & Range $^{a}$ & $\mathrm{R}^{2}$ & $0,50^{a}$ & $7,5^{\text {a }}$ & $15^{\text {a }}$ & $0,50^{a}$ & $7,5^{a}$ & $15^{a}$ \\
\hline 3HYPYR & 1,59 & $0,50-20$ & 0,9968 & 6,9 & 5,4 & 3,8 & 8,3 & 7,0 & 4,7 \\
\hline 2FPRZ & 2,07 & $0,50-20$ & 0,9991 & 7,1 & 5,2 & 4,4 & 7,4 & 6,6 & 5,4 \\
\hline 4HYBZOH & 2,32 & $0,50-20$ & 0,9973 & 6,3 & 4,8 & 4,0 & 7,2 & 5,7 & 5,7 \\
\hline 2HY6MPYR & 2,86 & $0,50-20$ & 0,9978 & 6.2 & 5,1 & 4,5 & 8,0 & 6,7 & 5,6 \\
\hline PYR & 3,21 & $0,50-20$ & 0,9982 & 5,9 & 5,2 & 4,4 & 7,6 & 6,4 & 5,1 \\
\hline 2CIPRZ & 3,94 & $0,50-20$ & 0,9994 & 5,2 & 4,8 & 3,8 & 7,8 & 6,0 & 5,5 \\
\hline 4METPYR & 4,64 & $0,50-20$ & 0,9982 & 6,4 & 5,5 & 4,4 & 7,7 & 5,7 & 4,5 \\
\hline 2METPRZ & 5,05 & $0,50-20$ & 0,9975 & 6,2 & 5,2 & 4,1 & 8,1 & 6,1 & 4,8 \\
\hline 23MPRZ & 5,27 & $0,50-20$ & 0,9991 & 5,8 & 5,0 & 4,3 & 7,2 & 5,9 & 5,2 \\
\hline 34METBZOH & 6,37 & $0,50-20$ & 0,9987 & 7,0 & 5,4 & 3,8 & 7,8 & 6,3 & 4,7 \\
\hline 4METBZOH & 6,62 & $0,50-20$ & 0,9973 & 6,1 & 5,2 & 4,3 & 7,9 & 5,5 & 4,5 \\
\hline $4 \mathrm{CIBZOH}$ & 8,35 & $0,50-20$ & 0,9988 & 5,5 & 4,5 & 3,1 & 7,1 & 5,7 & 4,0 \\
\hline $26 \mathrm{CIBZOH}$ & 8,61 & $0,50-20$ & 0,9994 & 5,9 & 4,7 & 3,3 & 6,5 & 5,4 & 4,2 \\
\hline $26 \mathrm{MPRZ}{ }^{*}$ & 5,27 & $0,50-20$ & 0,9971 & 6,2 & 5,1 & 4,2 & 6,8 & 5,9 & 5,0 \\
\hline
\end{tabular}

${ }^{\mathrm{a}} \mathrm{mg} / \mathrm{L} ; *$ analysed individually with the same method

The same motivation was behind the development of a method for analysis of $12 \mathrm{HEXDIOL}$ and 16HEXDIOL as for the other tracer candidates. The goal was to analyse them in a fast and reliable manner in the same concentration range as the other 14 candidates. These two compounds are not suitable for analysis with UV detection, thus alternative techniques had to be considered. 
Historically, GC/FID or GC/ECD has been the chromatographic techniques of choice for analysis of low molecular weight (high volatility) alcohols. Because the samples to be analysed would be aqueous matrices, a sample pre-treatment technique had to be considered to avoid direct injection of water in the GC system. Additionally, even though the samples were originated by laboratory experiments, only a small volume of each (about $2 \mathrm{~mL}$ ) would be available. Thus, it was desirable to introduce an enrichment factor in the sample pre-treatment. With these considerations, solid-phase microextraction (SPME) becomes the most promising sample pre-treatment technique available. SPME consists of an adsorbent fibre, with many different available adsorbent phases, that will selectively adsorb the target compounds directly from the sample. It can be used by direct-immersion (DI) or headspace (HS) extraction, and the analytes are afterwards directly thermally desorbed in the GC's injection port. Increasing the aqueous sample salinity is a well-known way to reduce the solubility of organic molecules in water. This is known as the "salting-out effect" and is widely used in analytical chemistry, particularly in liquid-liquid extraction and SPME. Of the available SPME adsorbent phases, polydimethylsiloxane with divinylbenzene (PDMS-DVB) is widely reported, and suggested by the manufacturer, as the most efficient adsorbent for extraction of alcohols. For simplicity of the procedure at this stage, the GC system was coupled with flame ionisation detection (FID). Optimisation of the conditions of SPME extraction and chromatographic separation were performed and a method was successfully developed. The conditions were as follows:

- $\quad 1,5 \mathrm{~mL}$ of sample was saturated with $\mathrm{NaCl}$ in a $5 \mathrm{~mL}$ SPME vial.

- The closed vials containing the samples were then heated to 80 ${ }^{\circ} \mathrm{C}$. This step will facilitate the volatilisation of the analytes since DI-SPME was not possible with the available amount of sample.

- Headspace extraction with a PDMS-DVB SPME fibre was performed for 10 minutes. 
- The fibre was then desorbed in the injection port of the GC-FID system at $250{ }^{\circ} \mathrm{C}$ in splitless mode for 5 minutes.

- $\quad$ The GC-FID system used was a Varian 3800 equipped with a 30 m SGE HT5 capillary column (30 m X 0.5 mm X $0.25 \mu \mathrm{m})$.

- Helium at $1 \mathrm{~mL} / \mathrm{min}$ was used as carrier gas and the detector was set at $300{ }^{\circ} \mathrm{C}$.

- Chromatographic separation was obtained with a temperature ramping as follows:

- initially $50{ }^{\circ} \mathrm{C}$ for 2 minutes,

- followed by a heating step of $30^{\circ} \mathrm{C} / \mathrm{min}$ until $250^{\circ} \mathrm{C}$,

- and $250{ }^{\circ} \mathrm{C}$ kept for 5 minutes.

Figure 8 shows an example chromatogram of a sample with a mix of the tracer candidates at a concentration of $10 \mathrm{mg} / \mathrm{L}$ for the SPME-GC-FID method developed.

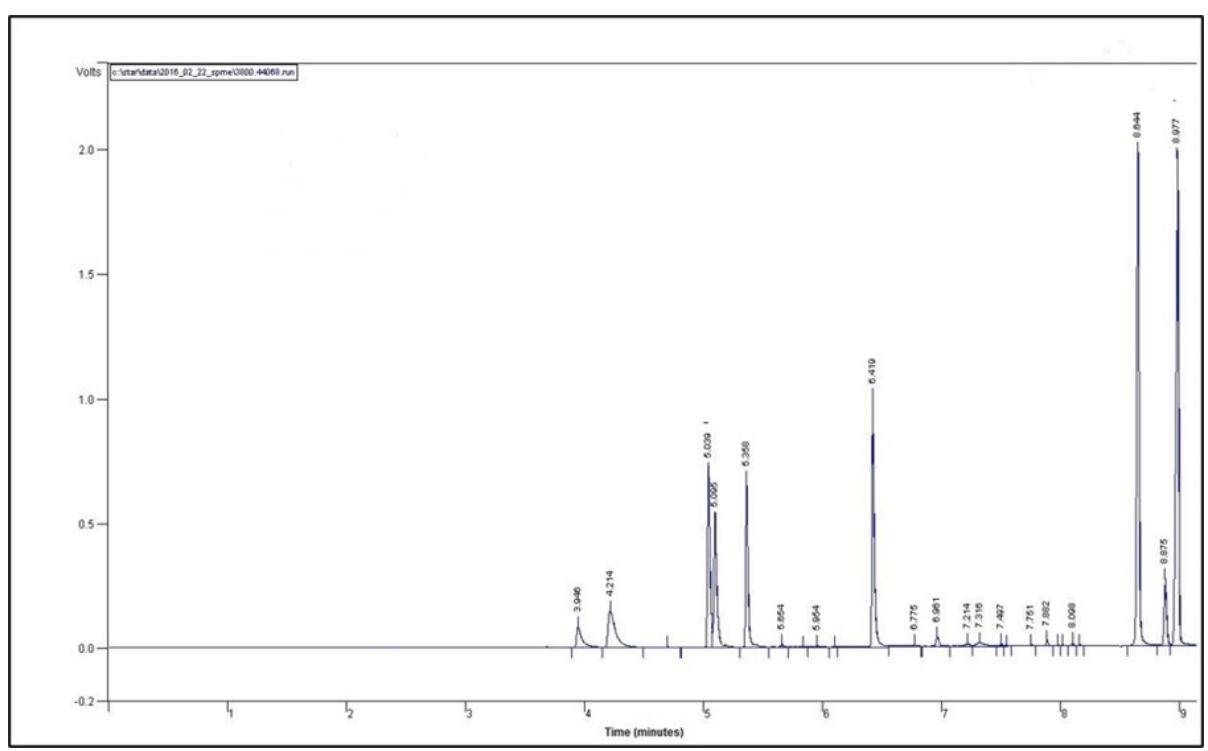

Figure 8. Example GC-FID chromatogram of a mixture of the tracers analysable by the method developed at a concentration of $10 \mathrm{mg} / \mathrm{L}$. Only 12HEXDIOL $\left(\mathrm{t}_{\mathrm{R}}=8,644 \mathrm{~min}\right)$ and $16 \mathrm{HEXDIOL}$ $\left(\mathrm{t}_{\mathrm{R}}=8,977 \mathrm{~min}\right)$ were determined by this method.

Notably, other of the compounds investigated in the present work could be analysed using the method developed, however it was only used for 
12HEDIOL and 16HEXDIOL. Similarly to the UPLC-UV method, the SPME-GC-FID method was calibrated between 0,5 and $20 \mathrm{mg} / \mathrm{L}$ and the linearity, inter $(\mathrm{N}=5)$ and intraday $(\mathrm{N}=7)$ precision, as well as the recovery evaluated in the same manner. Table 4 presents the chromatographic retention times (tR), linear range, and precision for each of the 2 tracer candidates analysed by the SPME-GC-FID method. Data about recovery, is presented in Table 5.

Table 4 - Retention time ( $\mathrm{t}_{\mathrm{R}}$ ), linear range, intra-day and inter-day precision for both PITT tracer candidates analysed by the GC-FID based method.

\begin{tabular}{|c|c|c|c|c|c|c|c|c|c|}
\hline \multirow{3}{*}{ Compounds } & \multirow{3}{*}{$t_{R}(\min )$} & & & \multicolumn{6}{|c|}{ Precision (\% RSD) } \\
\hline & & \multicolumn{2}{|c|}{ Linearity } & \multicolumn{3}{|c|}{ Intra-day precision } & \multicolumn{3}{|c|}{ Inter-day precision } \\
\hline & & Range a & $\mathrm{R}^{2}$ & $0,50^{a}$ & $7,5^{\mathrm{a}}$ & $15^{a}$ & $0,50^{a}$ & $7,5^{\mathrm{a}}$ & $15^{a}$ \\
\hline 12HEXDIOL & 8,644 & $0,50-20$ & 0,9981 & 8,2 & 6,1 & 4,3 & 8,0 & 6,5 & 4,1 \\
\hline 16HEXDIOL & 8,977 & $0,50-20$ & 0,9987 & 8,1 & 5,7 & 3,8 & 7,9 & 6,0 & 4,4 \\
\hline
\end{tabular}

${ }^{\mathrm{a}} \mathrm{mg} / \mathrm{L}$ 
Materials and methods

Table 5 - Recovery at three different levels of concentration for each of the 16 PITT tracer candidates $(\mathrm{N}=5$ ) (SPME-GC-FID was used to determine 12HEXDIOL and 16HEXDIOL and UPLC-UV for all other compounds).

\begin{tabular}{|c|c|c|c|}
\hline \multirow{2}{*}{ Tracers } & \multicolumn{3}{|c|}{ \%Recovery (\%RSD) } \\
\hline & Samples A $(0,5 \mathrm{mg} / \mathrm{L})$ & Samples B (7,5 mg/L) & Samples C (15 mg/L) \\
\hline 23MPRZ & $101(6,4)$ & $98,7(5,2)$ & $100(4,0)$ \\
\hline 26MPRZ & $103(5,9)$ & $101(5,3)$ & $99,4(3,5)$ \\
\hline 2METPRZ & $94,2(7,6)$ & $102(5,6)$ & $98,5(5,0)$ \\
\hline 2FPRZ & $101(5.0)$ & $101(3,5)$ & $99,1(2,8)$ \\
\hline 2CIPRZ & $104(5,5)$ & $98,2(4,3)$ & $99,1(3,7)$ \\
\hline $4 \mathrm{HYBZOH}$ & $94,3(6,1)$ & $97,6(4,6)$ & $101(4,1)$ \\
\hline $4 \mathrm{CIBZOH}$ & $97,0(5,1)$ & $102(4,4)$ & $100(3,0)$ \\
\hline $26 \mathrm{CIBZOH}$ & $103(6,4)$ & $100(2,8)$ & $99,7(3,4)$ \\
\hline 4METBZOH & $107(7,2)$ & $103(4,0)$ & $101(3,5)$ \\
\hline 34METBZOH & $95,3(6,3)$ & $101(4,2)$ & $98,4(3,7)$ \\
\hline PYR & $94,2(7,4)$ & $97,1(4,4)$ & $99,3(3,6)$ \\
\hline 4METPYR & $93,7(7,8)$ & $96,8(5,3)$ & $101(4,6)$ \\
\hline 3HYPYR & $104(6,6)$ & $100(4,7)$ & $102(3,9)$ \\
\hline 2HY6MPYR & $101(5,9)$ & $96,3(4,8)$ & $97,5(3,0)$ \\
\hline 12HEXDIOL & $97,2(4,7)$ & $101(4,2)$ & $98,5(3,9)$ \\
\hline 16HEXDIOL & $102(4,4)$ & $98,0(3,5)$ & $97,7(3,1)$ \\
\hline
\end{tabular}

\subsubsection{Analytical methods for laboratory samples - II}

The UPLC-UV method developed in section 2.3.1. was not effective for separation of 23MPRZ and 26MPRZ. With the development of the study, and for practical efficiency issues, it became necessary to develop an analytical method that would efficiently separate these two analytes. RP chromatography was still the separation technique used, but the column 
type and aqueous mobile phase composition were changed. All the other experimental conditions were the same as described in section 2.3.1.

The RP column used was an "Acquity UPLC BEH SHIELD" RP C18 carbamate $1.7 \mu \mathrm{m}$ packed column $(100 \mathrm{~mm} \mathrm{X} \mathrm{2,1} \mathrm{mm)} \mathrm{(Waters}$ Corporation, Milford, Massachusetts, USA). The major difference from this column from that previously used is the presence of carbamate groups in the $\mathrm{C} 18$ hydrocarbon phase that coats the packing particles. This increases the selectivity of the column for more polar compounds. The aqueous phase was modified for water at $\mathrm{pH} 9,0 \pm 0,1$ with NH3.

Figure 9 depicts an example chromatogram of a sample with a mixture of the relevant tracer candidates at a concentration of $10 \mathrm{mg} / \mathrm{L}$, obtained with the UPLC-UV method and Table 6 presents the tracers included in this method and respective retention times.

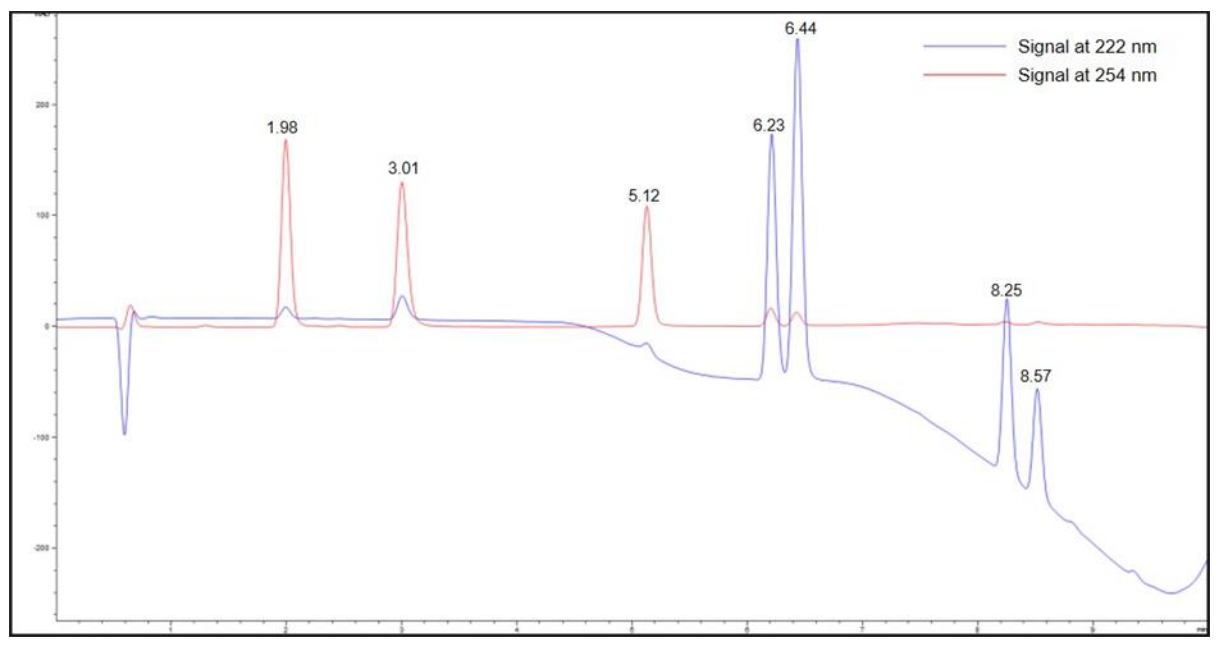

Figure 9. Example UPLC-UV chromatogram of a mixture of the relevant tracers analysable by the new method at a concentration of $10 \mathrm{mg} / \mathrm{L}$. Retention times in minutes are given on the top of the peaks. See Table 6 for identification of the peaks. 
Table 6 - Retention time $\left(t_{R}\right)$ for each of the PITT tracer candidates analysed by the new UPLCUV based method.

\begin{tabular}{lc}
\hline Compound & $\mathrm{t}_{\mathrm{R}}(\mathrm{min})$ \\
\hline 23MPRZ & 1,98 \\
26MPRZ & 3,01 \\
PYR & 5,12 \\
34METBZOH & 6,23 \\
4METBZOH & 6,44 \\
4ClBZOH & 8,25 \\
26ClBZOH & 8,57 \\
\hline
\end{tabular}

\subsubsection{Static stability experiments}

A PITT takes a relatively long time (anywhere between a few weeks to over 1 year), depending on the distance and volume swept between wells. Temperatures of the oilfields on the NCS can reach almost $150{ }^{\circ} \mathrm{C}$. The experiments in the present section were therefore devised to evaluate long term stability in a range between $25{ }^{\circ} \mathrm{C}$ and $150{ }^{\circ} \mathrm{C}$ for up to 12 weeks. Berea sandstone, limestone and kaolinite were also used in these experiments to assess their influence on the tracer candidates. Berea sandstone, limestone and kaolinite were used as "model" rock materials for sedimentary rock basins containing hydrocarbons. These materials are not necessarily representative of every possible oil-bearing formation. However, testing the PITT tracer candidates with rocks from all oilfields on the NCS would be an impossible task in a development program like this one. Furthermore, these stability experiments are designed to characterise and evaluate primarily thermal breakdown and chemical interactions like sorption to a rock substrate or substratecatalysed chemical breakdown. Thus, the rock materials used 
approximated reality as they contain most of the same mineralogy and geochemistry as encountered in conventional reservoirs. A description of the experiments is presented hereafter.

Test tubes for incubation of the samples were obtained by cutting $50 \mathrm{~cm}$ long durex glass tubes (i.d. $6 \mathrm{~mm}$, o.d. $9 \mathrm{~mm}$ ) from $1.5 \mathrm{~m}$ tubes (Schott AG, Mitterteich, Germany). The tubes were then thermally sealed on one end. The tubes prepared in this way were submitted to thermal decontamination/healing at $550{ }^{\circ} \mathrm{C}$ during 15 minutes under normal atmosphere.

A selected amount of PITT tracer candidates was dissolved in $1 \mathrm{~L}$ of brine with the ion composition given in Table 7. The solution was then sonicated at room temperature and simultaneously sparged with argon at $5 \mathrm{~mL} / \mathrm{min}$ for 20 minutes to remove the dissolved air. Three different brines were prepared at different initial $\mathrm{pH}$ values $(5,5 ; 7,1$ and 8,0$)$. The removal of oxygen and the $3 \mathrm{pH}$ values were used to mimic realistic conditions, where most produced waters have no oxygen dissolved and the $\mathrm{pH}$ values are in the range covered by the present study (Fakhru'lRazi et al., 2009). The PITT is designed for mature waterflooded oilfields. On the NCS the produced waters are a mixture of formation water with injection sea water, typically with an ion composition closer to sea water. Worldwide, a huge variation can be observed in the ion composition of produced waters. This study, however, was based on the conditions of the NCS. The salinity conditions used combined with the duration of the experiments allowed to evaluate the effects of elevated salinity on the properties of the tracer candidates under study.

$2 \mathrm{~mL}$ of solution of the tracer candidates, kept under constant sparging with argon at $5 \mathrm{~mL} / \mathrm{min}$, were transferred to each tube in four sets of test tubes. One set of test tubes had no substrate added, and the other 3 sets contained $600 \mathrm{mg}$ of powder $(125 \mu \mathrm{m}-250 \mu \mathrm{m})$ of Berea sandstone, kaolinite, and limestone, respectively. This amount of rock materials was selected to create a high surface area seen by the tracer candidate, while at the same time ensuring a homogenous slurry with the smooth agitation used. 
Materials and methods

Table 7 - Composition of the brine used in the experiments (from Paper III).

\begin{tabular}{lc}
\hline \multicolumn{1}{c}{ Salt } & Concentration $(\mathrm{g} / \mathrm{L})$ \\
\hline $\mathrm{NaCl}$ & 36,855 \\
$\mathrm{KCl}$ & 0,629 \\
$\mathrm{CaCl}_{2} \cdot 2 \mathrm{H}_{2} \mathrm{O}$ & 3,814 \\
$\mathrm{MgCl}_{2} \cdot 6 \mathrm{H}_{2} \mathrm{O}$ & 2,550 \\
$\mathrm{BaCl}_{2} \cdot 2 \mathrm{H}_{2} \mathrm{O}$ & 0,088 \\
$\mathrm{SrCl}_{2} \cdot 6 \mathrm{H}_{2} \mathrm{O}$ & 0,437 \\
$\mathrm{NaHCO}_{3}$ & $0,157 *$ \\
$\mathrm{Na}_{2} \mathrm{SO}_{4}$ & 0,046 \\
\hline
\end{tabular}

* $\mathrm{NaHCO} 3$ was used as $\mathrm{pH}$ buffer in varying amounts $(0,157 \mathrm{~g} / \mathrm{L}$ for initial $\mathrm{pH}$ of 7,$1 ; 0.280 \mathrm{~g} / \mathrm{L}$ for initial $\mathrm{pH}$ of 8,0; no $\mathrm{NaHCO} 3$ for initial $\mathrm{pH}$ of 5,5).

All test samples were prepared in triplicate. The test tubes containing the samples were then thermally sealed under vacuum and incubated in different thermal cabinets set at $25^{\circ} \mathrm{C}, 50{ }^{\circ} \mathrm{C}, 75^{\circ} \mathrm{C}, 100{ }^{\circ} \mathrm{C}, 125^{\circ} \mathrm{C}$ and $150{ }^{\circ} \mathrm{C}$. 3 sealed test tubes without substrate were selected to determine the exact initial concentrations of the tracer candidates. Every heating cabinet had a rotation mechanism incorporated to ensure smooth agitation during the test. Figure 10 is a picture of one of the thermal cabinets with the sealed tubes containing the samples under testing. These experiments were performed at three different initial $\mathrm{pH}$ values: 5.5, 7.1 and 8.0. The concentration of the tracer candidates in the tested samples was determined after 1, 3, 6 and 12 weeks of incubation using the analytical methods described in section 2.3.1. pH was also measured at the end of the incubation period.

The experiments performed in this section had an "eliminatory" character, and only the PITT tracer candidates with relevant long-term stability and absence of notable interactions with the rock materials were selected for further experiments. 


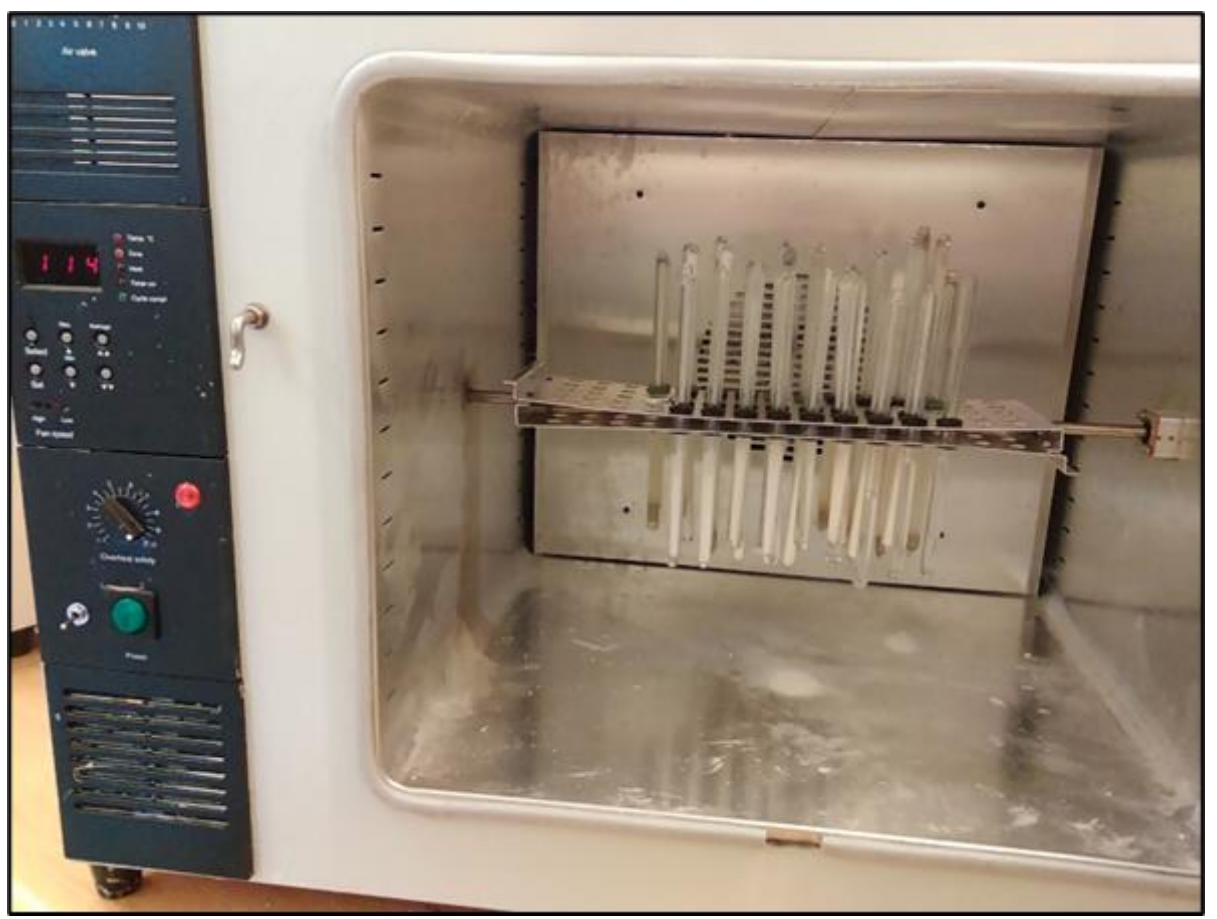

Figure 10. Picture of the samples tested inside one of thermal cabinets with a rotation mechanism.

\subsubsection{Analytical methods for field samples}

Focusing of the tracers qualified by the stability experiments in section 2.3.3., an analytical method was developed for the identification and quantification of the oil/water partitioning tracer candidates in real produced water samples from the NCS. This method intended to quantify the candidate compounds in the lowest possible concentration and is a central part of the present thesis, constituting by itself one of the chapters (Paper VI). For this reason, the development of the method will not be presented in this section, only a summary will be given.

The method developed for analysis of the PITT tracer candidates in real production waters is based on GC coupled with tandem-MS after a cleaning/concentration of the samples with a sequential DI-HS-SMPE using a divinylbenzene-carboxen-polydimethylsiloxane (DVB/CAR/PDMS) fibre. The conditions of the optimised method are 
as follows and produced LOQs in the low ng/L range for every tracer candidate:

- $5 \mathrm{~mL}$ of sample at $\mathrm{pH} 9.0$ with $1.8 \mathrm{~g}$ of $\mathrm{NaCl}$, constant stirring,

- 5 minutes of DI-SPME followed by 15 minutes of HS-SPME at $70{ }^{\circ} \mathrm{C}$ using a DVB/CAR/PDMS $(50 / 30 \mu \mathrm{m})$ fibre.

- Desorption of the fibre during 5 minutes in the injection port at $250{ }^{\circ} \mathrm{C}$ in splitless mode for 2 minutes.

- The chromatographic separation was achieved using a Thermo Scientific Trace 1310 gas chromatograph (Thermo Fischer Scientific, Waltham, MA, USA) equipped with a Restek Rtx®5MS column ( $30 \mathrm{~m} \mathrm{X} 0.25 \mathrm{~mm}$ X $0.25 \mu \mathrm{m})$.

- The temperature program of the oven was as follows:

- initial temperature $50{ }^{\circ} \mathrm{C}$ kept for 3 minutes,

- followed by a ramp of $20^{\circ} \mathrm{C} / \mathrm{min}$ to $110^{\circ} \mathrm{C}$,

- and another ramp of $15^{\circ} \mathrm{C} / \mathrm{min}$ to $290^{\circ} \mathrm{C}$,

- finally, 7 minutes at $290{ }^{\circ} \mathrm{C}$.

- Helium with a purity of $99.999 \%$ (Praxair Norway AS, 0663 Oslo) was used as carrier gas at a constant flow of $1 \mathrm{~mL} / \mathrm{min}$.

- The chromatographic system was coupled to a triple quadrupole mass spectrometer Thermo Scientific TSQ 8000 (Thermo Fischer Scientific, Waltham, MA, USA).

- The temperature of the ion transfer line and ion source were 290 ${ }^{\circ} \mathrm{C}$ and $320{ }^{\circ} \mathrm{C}$, respectively.

- The MS was operated in electron impact (EI) ionization mode $(+70 \mathrm{eV})$ and selected reaction monitoring (SRM) was used to monitor 3 transitions for identification of each of the target compounds, and quantification done with one of those transitions.

\subsubsection{Characterisation of the $K$-value}

The experiments presented hereafter intended to verify the existence of phase partitioning (a real solution of the tracer candidates in a hydrocarbon and an aqueous phase), to determine the influence of 
hydrocarbon composition, temperature, and salinity and ion composition of the aqueous phases on the value of $\mathrm{K}$, as well as to evaluate the reversibility of partitioning.

These data are extremely important as they can be used to correct the $\mathrm{S}_{\mathrm{OR}}$ values obtained in field tests, whether Eq. 3 is used for direct calculation from the same mass production landmark of the curve, or simulators incorporate the tracer production curves. Known data about the interwell region can be used to correct the possible variations of the K-value used in the Sor determination. The experiments were performed both with a synthetic mixture of hydrocarbons and real black oils from different fields on the NCS. The synthetic mixture of hydrocarbons was used as it simplifies the verification of the existence of partitioning. Synthetic mixtures also allow for testing of the effect of changing aromaticity of the oil on the partitioning of tracer candidates. The description of the experiments follows below in a simplified form. A more thorough description can be found in Paper VII. These experiments were performed on 7 of the initial PITT tracer candidates that had survived the stability experiments: PYR, 23MPRZ, 26MPRZ, 4METBZOH, 34METBZOH, 4ClBZOH and 26ClBZOH.

All experiments were based on the so called "shake flask method". This method consists of the mixing of two immiscible phases with the solute candidate tracer dissolved in one of them. After partition equilibrium and subsequent phase separation has been reached, the concentrations of the solutes were measured in one or both phases. In all experiments the hydrocarbon phase and the aqueous phase to be used in the binary system were mutually saturated with each other before partition experiments with the solutes commenced. Only after this process the compounds under study were added (typically to the aqueous phase) by dilution of aliquots from a mother solution of known concentration. This step consisted of adding small volumes (between $500 \mu \mathrm{L}-1 \mathrm{~mL}$ ) of the concentrated solution of the tracers to $500 \mathrm{~mL}$ of the aqueous phase previously saturated with the organic phase. 
Two aqueous solutions were prepared containing the seven studied compounds, each at a concentration of $10 \mathrm{mg} / \mathrm{L}$, with salinities composed of $2,5 \% \mathrm{NaCl}$ and $5,0 \% \mathrm{NaCl}(\mathrm{m} / \mathrm{V})$, respectively. 5 aliquots were taken of each of the solutions and their $\mathrm{pH}$ was adjusted to 5, 6, 7, 8 and 9, respectively, by adding either $\mathrm{HCl}$ or $\mathrm{NaOH}$. A synthetic hydrocarbon "model oil" solution composed of $20 \%$ (vol.) toluene, $70 \%$ (vol.) isooctane and $10 \%$ (vol.) octanol was prepared. An aliquot of $15 \mathrm{~mL}$ was taken from each of the aqueous solutions at the different $\mathrm{pH}$ values containing the studied compounds and placed in $20 \mathrm{~mL}$ glass vials with aluminium caps containing a PTFE septum. $5 \mathrm{~mL}$ of the model oil were added to each of the vials. The mixture was thoroughly shaken and placed in a thermal cabinet at $25{ }^{\circ} \mathrm{C}$ for $24 \mathrm{~h}$. After $24 \mathrm{~h}$, a $2 \mathrm{~mL}$ sample was taken from the aqueous phase of each vial using a syringe with a 24 ga needle. The concentration of the tracer compounds was then determined in these samples. All experiments were performed in triplicate. Using a mass balance, the concentration of the compounds in the hydrocarbon phase can be determined.

Subsequently, solutions of the potential tracer compounds under study were prepared at a concentration of $10 \mathrm{mg} \mathrm{L}-1$ in deionized water, 2,5\% $\mathrm{NaCl}, 5,0 \% \mathrm{NaCl}, 7,5 \mathrm{NaCl}$ and $10 \% \mathrm{NaCl}$. The $\mathrm{pH}$ values of these solutions were adjusted to $8,0 \pm 0,1$ by adding $280 \mathrm{mg} / \mathrm{L}$ of $\mathrm{NaHCO} 3$. A model oil composed of $20 \%$ toluene (vol.) and varying fractions of octanol (0\% - 20\% vol.) and iso-octane (60\% - $80 \%$ vol) was used as the hydrocarbon phase. The purpose of using such mixtures was to evaluate the effect of polarity and "nature" of the organic phase (more aromatic or more aliphatic) on the partitioning of the tested compounds. Series of $15 \mathrm{~mL}$ of each of the tracer solutions were transferred to $20 \mathrm{~mL}$ vials with aluminium caps with PTFE septum together with $5 \mathrm{~mL}$ of the various model oil mixtures. These mixtures were shaken and placed in thermal cabinets at $25{ }^{\circ} \mathrm{C}, 50{ }^{\circ} \mathrm{C}, 75^{\circ} \mathrm{C}$ and $100{ }^{\circ} \mathrm{C}$ respectively to evaluate the effect of temperature on the partitioning. After $24 \mathrm{~h}$, samples of the aqueous phases were taken, and the concentration of the tracers determined by the method developed in section 2.3.2. This procedure 
was also applied to samples of real black oils from various fields on the NCS.

To verify the reversibility of the partitioning and its independence of initial concentration, so-called "stripping" or back-extraction experiments were performed. Series of samples of $2 \mathrm{~mL}$ of the model oil solutions containing the chemical compounds from the experiments above were sampled and transferred to $10 \mathrm{~mL}$ vials with aluminium caps with PTFE septum. $5 \mathrm{~mL}$ of the previously prepared solutions of $2,5 \%$ $\mathrm{NaCl}, 5,0 \% \mathrm{NaCl}, 7,5 \mathrm{NaCl}$ and $10 \% \mathrm{NaCl}$ respectively were mixed with the $2 \mathrm{~mL}$ organic phase and the shaking, incubation and analytical processes repeated.

Many of the results obtained for 2 of the compounds $(4 \mathrm{ClBZOH}$ and $34 \mathrm{METBZOH})$ required re-testing. $\mathrm{K}$-values for $4 \mathrm{ClBZOH}$ exhibited sometimes very high values and the opposite was observed for 34METBZOH. In both cases, an accurate determination of the K-value could be compromised if experimental adjustments were not done, as variations in the concentrations of these compounds could not be accurately determined. The same procedure described in the present point was employed with different initial concentrations of the two compounds in solution. When required, the initial concentrations of $4 \mathrm{ClBZOH}$ and $34 \mathrm{METBZOH}$ were of $200 \mathrm{mg} / \mathrm{L}$ and $1 \mathrm{mg} / \mathrm{L}$, respectively.

To examine the effect of replacing monovalent ions with divalent ions holding the ion strength constant, the experiments performed as described in 2.3.2. were repeated using a fixed "model oil" mixture composed of $80 \%$ (vol) iso-octane and $20 \%$ (vol) toluene. The aqueous solutions of the partitioning compounds were prepared in a mixed solution of monovalent and divalent ions building up the same ionic strength (I) as the ones using only $\mathrm{NaCl}$. Equation 4 was used to calculate I (expressed in M).

$$
\mathrm{I}=\frac{1}{2} \sum_{i=1}^{n} C_{i} Z_{i}^{2}
$$


In equation $4, \mathrm{C}_{\mathrm{i}}$ is the molar concentration of ion $\mathrm{i}(\mathrm{M}), \mathrm{Z}_{\mathrm{i}}$ is the charge number of that same ion, and $\mathrm{n}$ is the number of different ions.

The amount of $\mathrm{NaCl}$ present in each solution was reduced to $1 / 4$, and $\mathrm{Na}_{2} \mathrm{SO}_{4}, \mathrm{CaCl}_{2}$ and $\mathrm{MgCl}_{2}$ were added in sufficient amounts to reach the same values of I. Table 8 presents the amounts of each salt used and I of each solution. All experiments were performed with 3 real replicates.

Table 8 - Salts, respective amount present, and I of the aqueous solutions of the studied compounds (Paper VII).

\begin{tabular}{|c|c|c|c|c|c|c|c|c|}
\hline $\begin{array}{c}\mathrm{C} . \\
\mathrm{NaCl} \\
(\mathrm{g} / \mathrm{L}) \\
\end{array}$ & $\begin{array}{c}\mathrm{C} . \\
\mathrm{NaCl} \\
(\mathrm{M})\end{array}$ & $\begin{array}{c}\mathrm{C} . \\
\mathrm{Na}_{2} \mathrm{SO}_{4} \\
(\mathrm{~g} / \mathrm{L})\end{array}$ & $\begin{array}{c}\mathrm{C} . \\
\mathrm{Na}_{2} \mathrm{SO}_{4} \\
(\mathrm{M})\end{array}$ & $\begin{array}{c}\mathrm{C} . \\
\mathrm{CaCl}_{2} \\
(\mathrm{~g} / \mathrm{L})\end{array}$ & $\begin{array}{c}\mathrm{C} . \\
\mathrm{CaCl}_{2} \\
(\mathrm{M})\end{array}$ & $\begin{array}{c}\mathrm{C} . \\
\underset{\mathrm{MgCl}}{(\mathrm{g} / \mathrm{L})} \\
\end{array}$ & $\begin{array}{c}\mathrm{C} . \\
\mathrm{MgCl}_{2} \\
\text { (M) }\end{array}$ & $\underset{(\mathbf{M})}{\mathbf{I}}$ \\
\hline 25 & 0,43 & 0 & 0 & 0 & 0 & 0 & 0 & 0,43 \\
\hline 50 & 0,86 & 0 & 0 & 0 & 0 & 0 & 0 & 0,86 \\
\hline 75 & 1,28 & 0 & 0 & 0 & 0 & 0 & 0 & 1,28 \\
\hline 100 & 1,71 & 0 & 0 & 0 & 0 & 0 & 0 & 1,71 \\
\hline 6,25 & 0,11 & 2,0 & 0,014 & 5,1 & 0,046 & 4,4 & 0,046 & 0,43 \\
\hline 12,5 & 0,22 & 2,0 & 0,014 & 11,1 & 0,10 & 9,5 & 0,10 & 0,86 \\
\hline 18,75 & 0,32 & 2,0 & 0,014 & 17,0 & 0,15 & 14,6 & 0,15 & 1,28 \\
\hline 25 & 0,43 & 2,0 & 0,014 & 23,0 & 0,21 & 19,7 & 0,21 & 1,71 \\
\hline
\end{tabular}

\subsubsection{Flooding experiments}

The main goal of the flooding experiments was to verify if the PITT tracer candidates exhibit the desired behaviour relatively to a passive water tracer mimicking the water flow as close as possible, while traveling through a consolidated sedimentary porous rock. The reference water tracer used was tritiated water (HTO). Tritiated water is water with one of the hydrogen atoms substituted by a tritium atom and has virtually the same physico-chemical properties as water itself. Tritium is a radioactive isotope of hydrogen with a low-energy $\beta$-decay and a halflife of 12,32 years (Lucas and Unterweger, 2000). HTO occurs naturally 
in very small amounts, thus its background is virtually non-existent in aqueous solutions. HTO is easily detected by liquid-scintillation counting (LSC). Figure 11 depicts a simplified scheme of a LSC instrument.

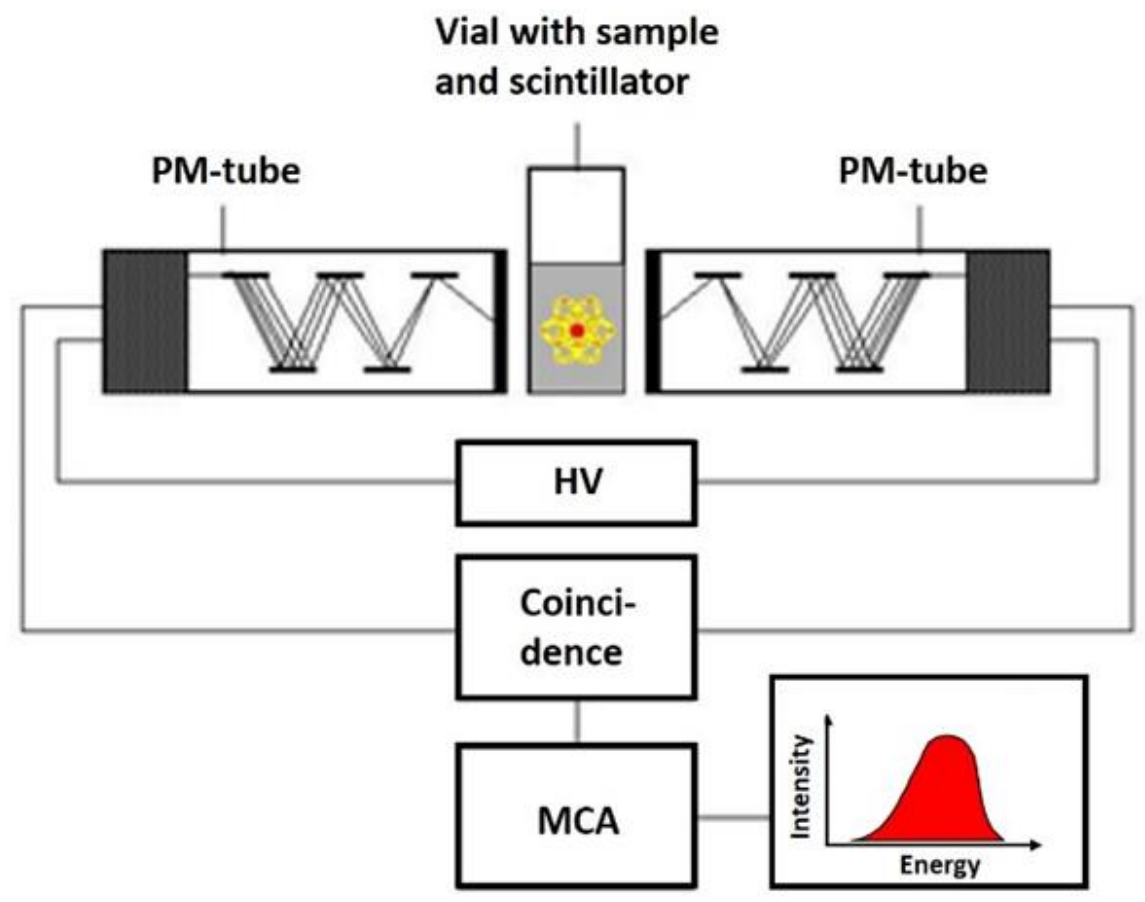

Figure 11. Simplified scheme of HTO analysis in a LSC instrument

The vial contains the liquid radioactive sample intimately mixed with a scintillation cocktail composed of fluorescent molecules. The radioactivity, for instance the emission of $\beta$-particles, leads to isotropic emission of a shower of photons for each $\beta$-particle. The photons are registered by two photomultipliers (PM-tubes), and the coincidently detected signals sent to a multichannel analyser (MCA) for creating the energy spectrum of the radiation from the radioactive substance. The energy distribution of the obtained spectrum is characteristic of a given 
radionuclide. Figure 12 shows the energy spectra of tritium in the form of HTO.

The number of net counts (gross counts minus the background) is directly proportional to the amount of HTO present and the measurement can be optimised by selecting the maximum energy channels to integrate.

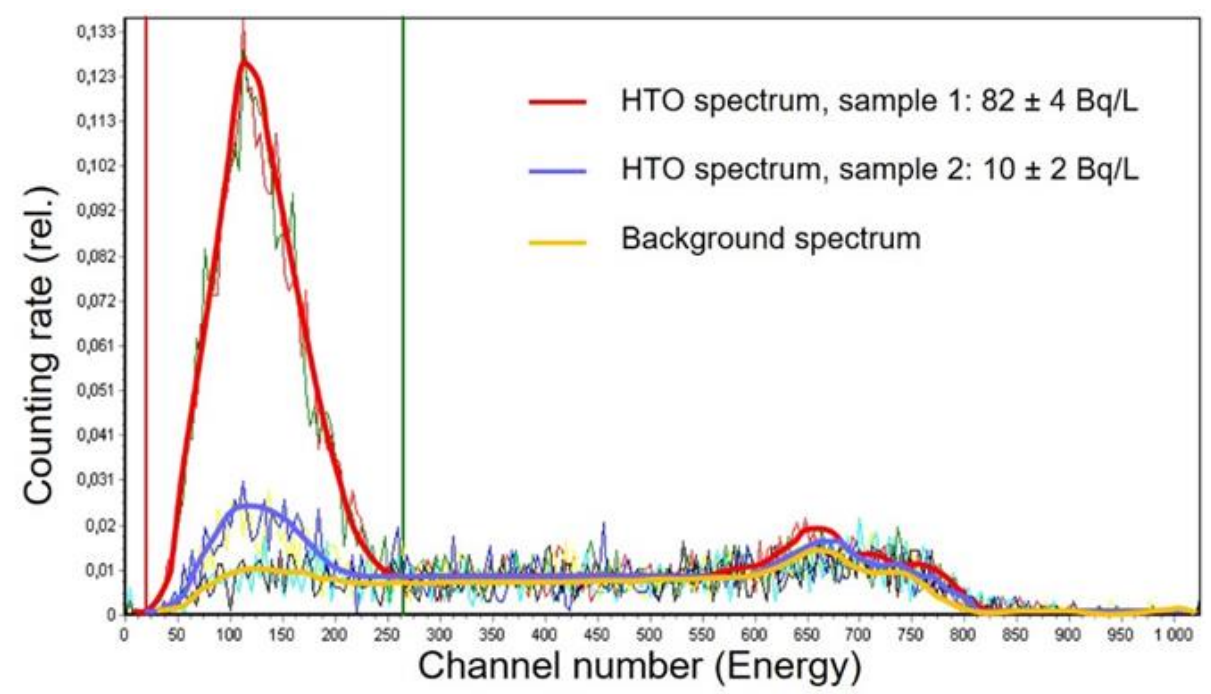

Figure 12. Energy spectra of tritium in the form of tritiated water at two different activity concentrations: $82 \pm 4 \mathrm{~Bq} / \mathrm{L}$ (red curve) and $10 \pm 2 \mathrm{~Bq} / \mathrm{L}$ (blue curve), together with a background spectrum (yellow curve).

Berea sandstone cores and Stevns Klint chalk cores, both water and oil saturated, were used in the experiments. The dimensions of these cylindrical cores were as follows: $38,1 \mathrm{~mm}$ diameter x $70 \mathrm{~mm}$ length. The cores were coated with polyurethane and placed in a high-pressure Hassler cell. The cell was then connected to a vacuum pump operating at approximately $0,1 \mathrm{mbar}$ and degassed. The inlet and outlet valves were closed, and the Hassler cell placed inside a cabinet with thermal control. Figure 13 illustrates a scheme of the experimental setup used for the flooding experiments. Next, the experimental procedure for the core flooding experiments is described. 


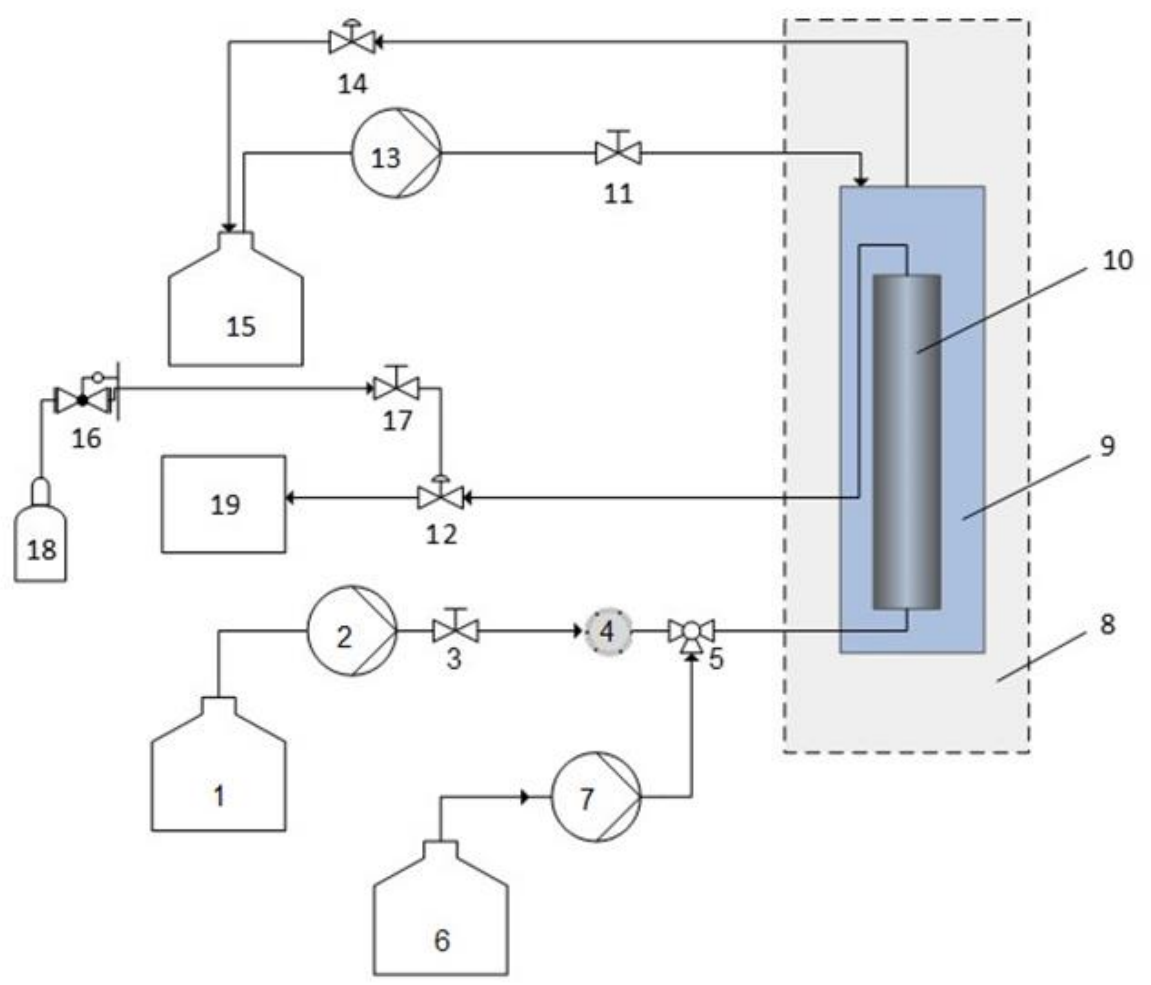

Figure 13. Scheme of the experimental setup used in the flooding experiments

Legend:

1 - Reservoir of injection brine

11 - Manual isolation valve

2 - Brine Injection pump

12 - Back pressure regulator

3 - Manual isolation valve

13 - Core plug pressure pump

4 -6-way injection loop

14 - Back pressure regulator

$5-3$ way plug valve

15 - Reservoir of deionised

6 - Reservoir of oil water (for core plug pressuring)

7 - Oil injection pump

16 - Pressure regulator

8 - Thermal cabinet

17 - Manual isolation valve

9 - Hassler cell

18 - Pressurised nitrogen

10 - Polyurethane core plug 
Identical procedures were adopted for the sandstone and chalk cores. The flooding experiments were performed at $70{ }^{\circ} \mathrm{C}$. Water saturation was obtained by pumping brine through the cores at $2 \mathrm{~mL} / \mathrm{min}$ for 2 days. After this period, the injection rate was reduced to $0,1 \mathrm{~mL} / \mathrm{min}$ and pressure of injection set to 140 bar. These values were maintained constant throughout the experiments. Pressurised water at 170 bar was used as the confining pressure around the core plug inside the Hassler cell. The dead volume of the setup was previously determined to be 3,62 $\mathrm{mL}$ by calculus using the tube lengths and inner diameters, as well, as the dead volumes of valves and connectors supplied by the manufacturers. The brine used was the same as presented in Table 7. A mixed solution of the relevant PITT tracer candidates at $500 \mathrm{mg} / \mathrm{L}$ was prepared in the brine. $2 \mathrm{~mL}$ of the tracer solution were spiked with $10 \mu \mathrm{L}$ of a "stock" HTO solution. $250 \mu \mathrm{L}$ of the $500 \mathrm{mg} / \mathrm{L}$ tracer candidate solution spiked with HTO were loaded into the injection loop and the rest of the solution was used as reference to determine the recoveries from the flooding experiment. The $250 \mu \mathrm{L}$ were injected into the core as a pulse. This marked the beginning of the flooding experiment. All the produced fluid was collected by the auto-sampler in sample volumes of about $750 \mu \mathrm{L}$ each. The accurate volume of brine in each sample was determined by weighing the vials before and after the experiment. A total of 100 samples were taken and this marked the end of the core flooding experiment with a water saturated core. Black oil from the Heidrun field was used for the flooding experiments on cores prepared to Sor conditions. Sor was obtained as follows: after the end of the flooding experiments with the water saturated cores, black oil was injected for no less than 10 pore volumes. The system was the allowed to "mature" during 5 days at $70{ }^{\circ} \mathrm{C}$ with no fluid circulation. After this period, brine injection was initiated at a flowrate of $0,1 \mathrm{~mL} / \mathrm{min}$ and maintained until no visible hydrocarbons were produced (typically about 10 pore volumes). After this, the flooding experiments at $S_{\text {OR }}$ conditions were initiated. The procedure for injecting the tracer pulse and for collection of samples at the exit line was the as described for the 
experiments at the water saturated core. HTO and the PITT tracer candidates were analysed in all samples from each flooding experiment. The PITT tracer candidates were analysed by UPLC-UV with the method described in section 2.3.2. and the radioactivity of this solution was measured by LSC by mixing $50 \mu \mathrm{L}$ the sample with $5 \mathrm{~mL}$ of scintillator solution (mixture of fluorescent hydrocarbons).

When travelling through a porous medium, even with a small volume, the injected tracers will travel along slightly different paths (flow lines) with somewhat different linear velocity. Thus, different amounts of tracers will arrive at the producer at different times. The residence time distribution, $\mathrm{E}(\mathrm{t})$, is the function used to characterise this distribution. $\mathrm{E}(\mathrm{t})$ provides information about which fraction of the injected tracer was produced at a given time or time interval. It is defined by the concentration of tracer produced at a given moment, $\mathrm{C}(\mathrm{t})$, the production rate, $\mathrm{Q}(\mathrm{t})$, and the amount of tracer injected $(\mathrm{m})$ as:

$\mathrm{E}(\mathrm{t})=\frac{C(t) \cdot Q(t)}{m}$

In a closed system, where the total amount of injected tracer is recovered:

$\int_{-\infty}^{\infty} E(t) d t=1$

(Eq. 6)

In a system like the one used in the present study, where all the eluted mobile phase is collected in discrete samples, Eq. 6 can be rewritten as:

$\frac{\sum_{i}^{n} C_{i} Q_{i}}{m}=1$

Where $C_{i}$ is the concentration of tracer in sample $i, Q_{i}$ is the volume of sample $\mathrm{i}$, and $\mathrm{n}$ is the last sample were the tracer is quantifiable. Eq. 7 was used to determine the recovery of tracers in the flooding experiments. (Deans, 1978) developed a method for determining the total pore volume swept by a water tracer $\left(\mathrm{V}_{\mathrm{S}}\right)$ in a $1 \mathrm{D}$ core flooding experiment and assuming a completely layered model: 
$\mathrm{V}_{S}=\frac{\int_{0}^{\infty} C_{i} v_{i} d v}{\int_{0}^{\infty} C_{i} d v}$

(Eq. 8)

Where $\mathrm{v}_{\mathrm{i}}$ is the volume of the total eluted aqueous phase up to sample $\mathrm{i}$. This is equivalent to the expression for the first moment of the tracer production curve. Again, in our system all the eluted mobile phase was collected in discrete samples. Eq. 8 can be rewritten as:

$\mathrm{V}_{S}=\frac{\sum_{i}^{n} C_{i} v_{i} Q_{i}}{\sum_{i}^{n} C_{i} Q_{i}}$

(Eq. 9)

Eq. 9 was applied to the produced samples to determine the pore volume swept by the HTO pulse in both the water core and the core under SoR. In the water saturated core experiments, the ideal behaviour of the PITT tracer candidates should behave the same as passive water tracers. These compounds should only interact with hydrocarbon phases present in the porous medium and nothing else. Additionally, they should travel the same paths as water does. Thus, using Eq. 9 to determine the volume swept by the PITT tracer candidates vs the HTO reference is a good process to evaluate their dynamic properties. 


\section{Main results and discussion}

The main objective of the activities developed in the scope of the present study was to introduce and apply a methodology for selection and testing of new oil/water partitioning tracer candidates that leads to a minimisation of the risk of unsuccessful $S_{\text {OR }}$ determination in field tests. This is what justified the elimination of the candidates in different steps of the work developed. The core results and findings are presented briefly here hereafter by the order of the experimental steps that best suits the reasoning behind the development of the work. Reading of the articles in "Part 2" of this Thesis is advised.

\subsection{Static stability experiments}

The static stability experiments (see experimental details in chapter "2.3.3. Static stability experiments") produced eliminatory results. This means all the tracer candidates that do not exhibit the required long-term stability were discarded from subsequent qualification steps. The stability experiments produced, however, interesting results for several of the candidates that open other interesting possibilities for their use. The remaining fraction (RF) of the tracer candidates is defined as $\mathrm{C}(\mathrm{t}, \mathrm{T})$ at an experimental point divided by $\mathrm{C}$ (i). To facilitate the reading of most figures in the present section, error bars were deliberately omitted. Typical precision (standard deviation values associated with RF) of the experiments were in the range of $0,06-0,08$.

\subsubsection{Stability of pyrazines}

2,3-Dimethylpyrazine (23MPRZ) and 2,6-Dimethylpyrazine (26MPRZ) were found to be highly stable up to $150^{\circ} \mathrm{C}$ after 12 weeks of incubation. These two candidates are also insensitive to the presence of rock materials (kaolinite, sandstone, and limestone), and to the different $\mathrm{pH}$ conditions. All the variations observed in the RF values can be attributed to analytical uncertainty. This suggests these two PITT tracer candidates 
have the necessary stability to endure the harsh conditions encountered on the NCS oilfields. These were two of the oil/water partitioning tracer candidates selected for further application of the qualification methodology presented for new PITT tracers (Paper IV). Stability results for 23MPRZ and 26MPRZ as a function of time with temperature and $\mathrm{pH}$ as parameters are given in Figure 14 and Figure 15, respectively.

Corresponding results from the stability experiments on 2methoxypyrazine (2METPRZ), 2-chloropyrazine (2CIPRZ) and 2fluoropyrazine are given in Figure 16, Figure 17 and Figure 18, respectively. 

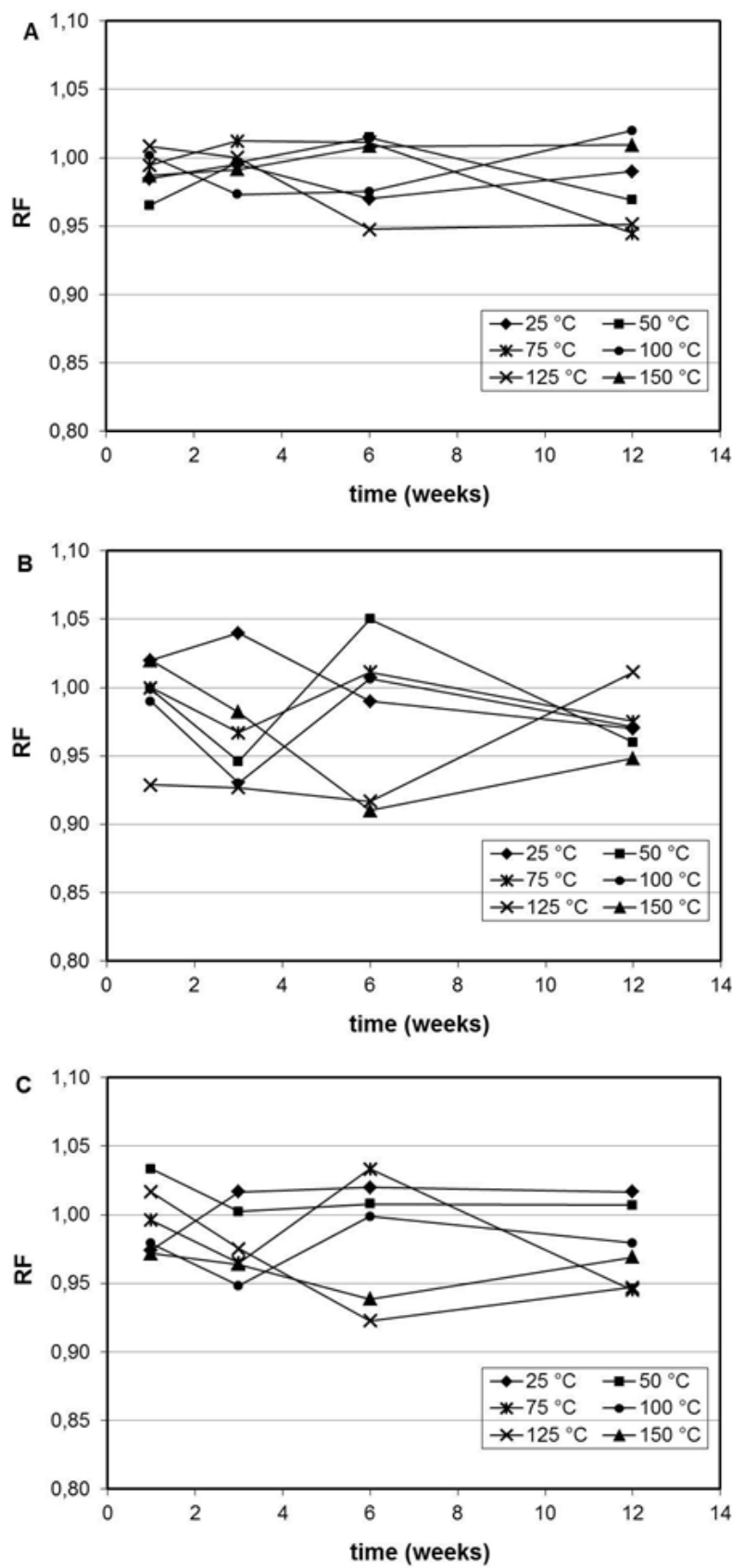

Figure 14. RF values of 2,3-Dimethylpyrazine as function of time and temperature of incubation. (A) Initial pH 5.5; (B) Initial $\mathrm{pH} 7.1$; (C) Initial $\mathrm{pH} 8.0$ and no rock substrate. 

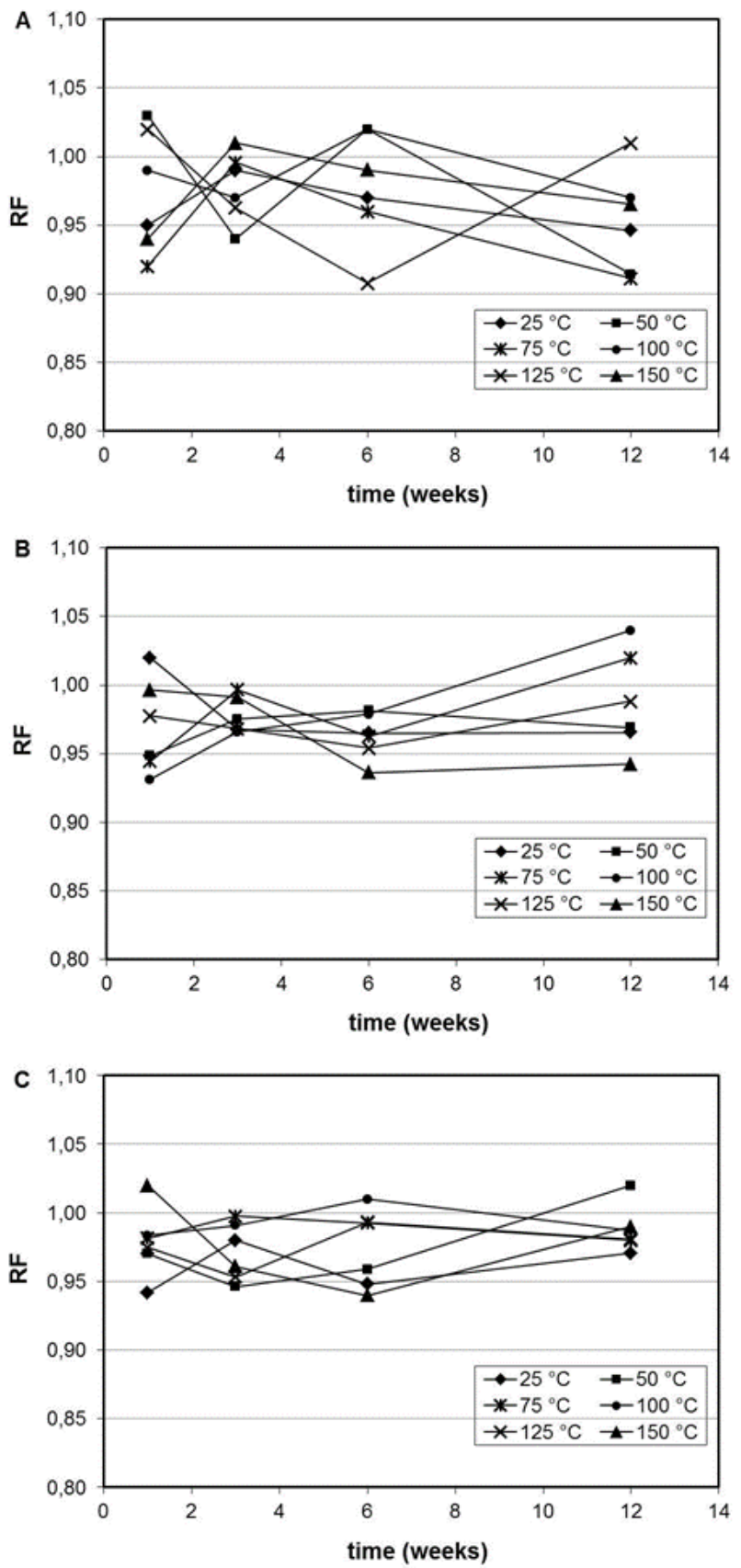

Figure 15. RF values of 2,6-Dimethylpyrazine as function of time and temperature of incubation. (A) Initial pH 5.5; (B) Initial pH 7.1; (C) Initial pH 8.0 and no rock substrate. 

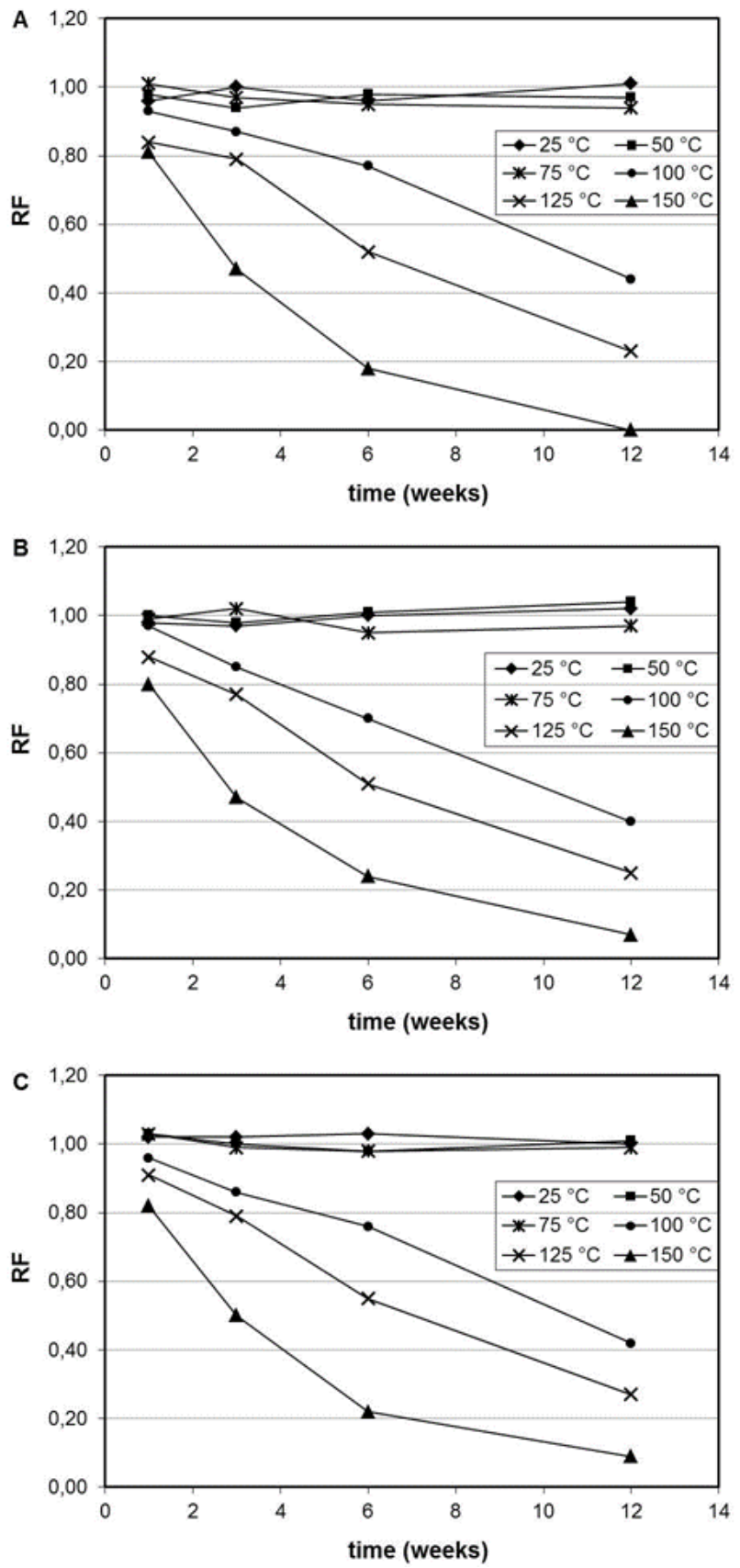

Figure 16. RF values of 2-methoxypyrazine as function of time and temperature of incubation. (A) Initial pH 5.5; (B) Initial pH 7.1; (C) Initial pH 8.0 and no rock substrate. 

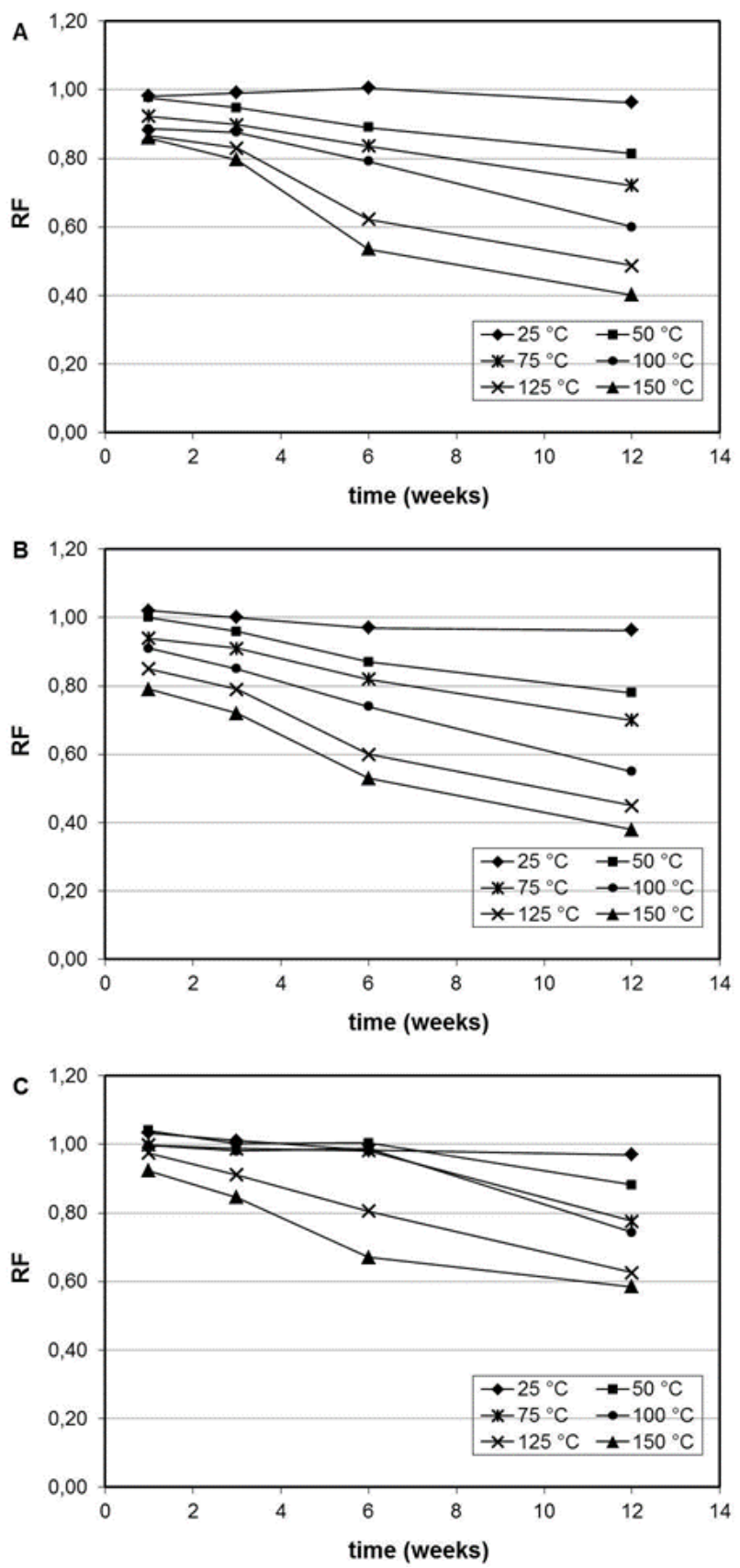

Figure 17. RF values of 2-chloropyrazine as function of time and temperature of incubation. (A) Initial $\mathrm{pH}$ 5.5; (B) Initial pH 7.1; (C) Initial pH 8.0 and no rock substrate. 

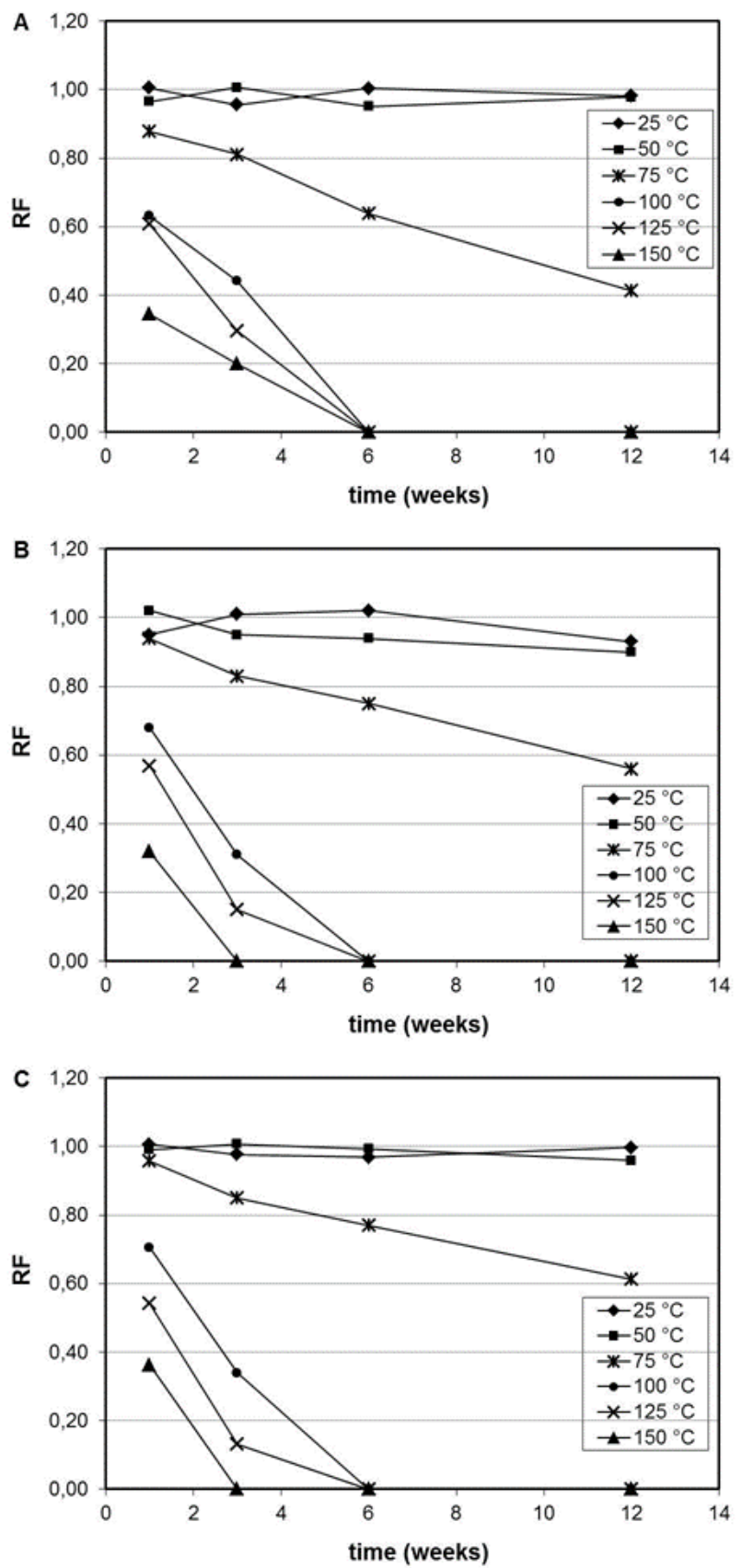

Figure 18. RF values of 2-fluoropyrazine as function of time and temperature of incubation. (A) Initial $\mathrm{pH}$ 5.5; (B) Initial $\mathrm{pH} 7.1$; (C) Initial $\mathrm{pH} 8.0$ and no rock substrate. 
Degradation products and kinetic models were not successfully found for 2CIPRZ and 2FPRZ. Results suggest that rate of degradation of 2CIPRZ and 2FPRZ is influenced by the $\mathrm{pH}$ value of the bulk. In many experimental points, the RF values appear to be higher as the $\mathrm{pH}$ value increases. However, the results (obtained at 3 different $\mathrm{pH}$ values) do not allow for a clear conclusion about this. Most of the data points where the $\mathrm{RF}$ values of 2-Cl-PRZ and 2-F-PRZ are higher at $\mathrm{pH} 8.0$ result from experiments performed at temperatures of $75^{\circ} \mathrm{C}$ and higher, however the differences observed are not sufficient to preserve the stability of the tracer candidates within a range which would allow considering them for field applications. (Paper IV).

The PITT tracer candidates were also incubated in the presence of Berea sandstone, limestone, and kaolinite to evaluate possible interactions with rocks characteristic of sedimentary oil reservoirs. The differences between the RF $(t, T)$ values in the stability experiments with rock substrate and the corresponding $\mathrm{RF}(\mathrm{t}, \mathrm{T})$ values without any rock substrate were evaluated and the maximum differences encountered are depicted in Fig. 19.

The presence of kaolinite severely reduces the RF of 2-MET-PRZ relatively to the experiments with no rock substrate. Results suggest that all other tracer candidates are "insensitive" to the presence of any of the tested rock materials. Kaolinite is well known to exhibit both adsorbent (Yu et al., 2013) and catalytic ( $\mathrm{Li}$ et al., 2019) activities. One of these phenomena is most likely responsible for the results obtained. However, no clear mechanism was identified by the present study (Paper IV). 

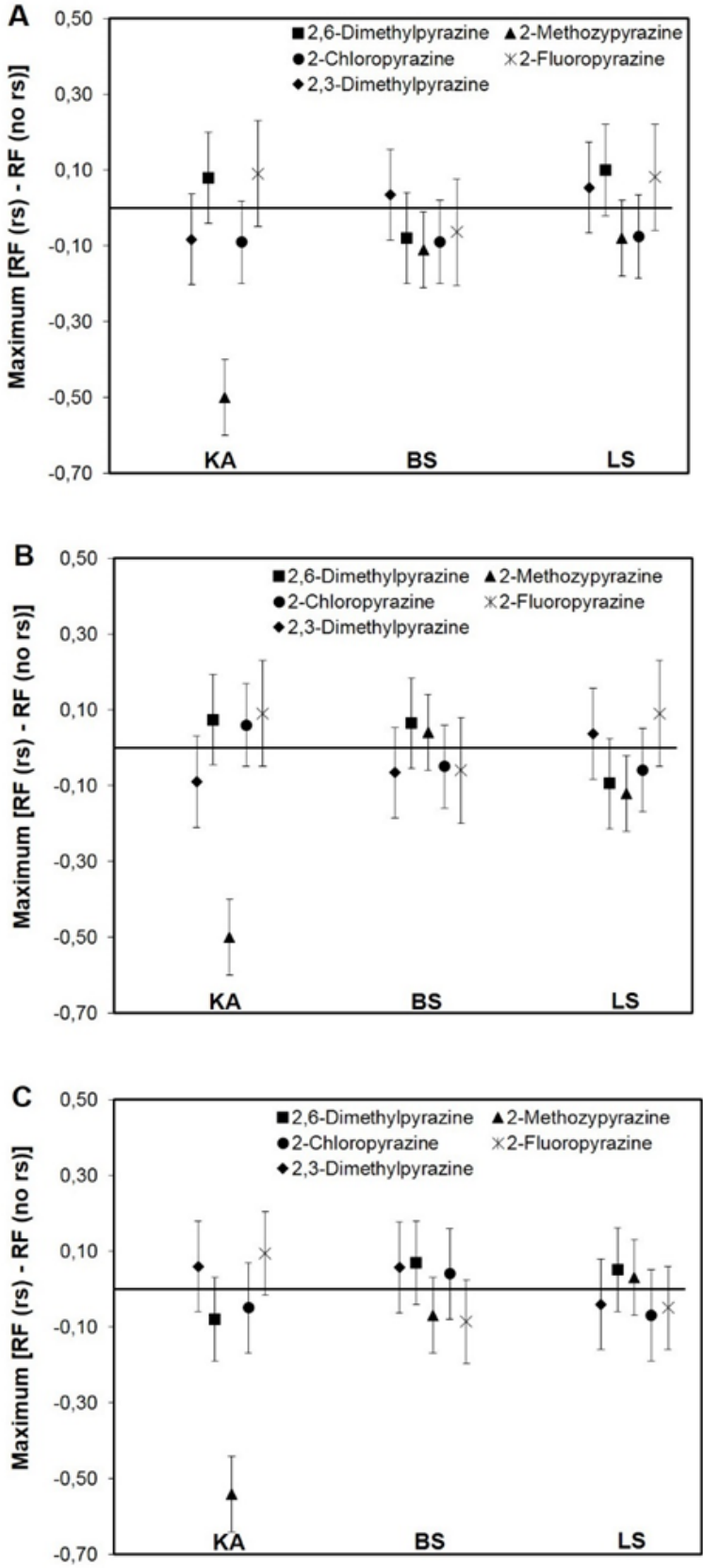

Figure 19. Maximum differences encountered in RF values between experiments with rock substrate (rs) and experiments without rock substrate $\pm 2 \sigma$ in RF (t, T). (KA) kaolinite, (BS) Berea sandstone, (LS) limestone. (A) Initial pH 5.5; (B) Initial pH 7.1; (C) Initial pH 8.0. 
Results show that the nature of the substituents (functional groups) plays a determinant role in the stability of a molecule. Only 2,3dimethylpyrazine and 2,6-dimethylpyrazine were investigated further after the stability experiments (Papers 6 and 7). These two compounds are, among the tested pyrazines, those with the less polar substituents (alkyl groups), which are also less reactive.

\subsubsection{Stability of benzyl alcohols}

The results of the stability experiments in this class of chemicals allows organising them in 3 groups: fully stable, partially stable, and unstable with other potential applications.

4-Chlorobenzyl alcohol (4ClBZOH) and 2,6-Dichlorobenzyl alcohol $(26 \mathrm{ClBZOH})$ are stable across the experimental conditions evaluated. They are also insensitive to the presence of rock materials and to $\mathrm{pH}$ variations within the tested range (Paper III). Stability results are displayed in Figure 20.

4-Methoxybenzyl alcohol (4METBZOH) and 3,4-dimethoxybenzyl alcohol (34METBZOH) exhibit a high stability at temperatures up to 125 ${ }^{\circ} \mathrm{C}$. However, at $150{ }^{\circ} \mathrm{C}$ some degradation is observable even after one week. Considering the stability of these two compounds at temperatures up to $125^{\circ} \mathrm{C}$, the observed values after this temperature "threshold" is most likely reflecting a purely temperature dependent degradation mechanism: the energy of the system reaches the necessary value to trigger degradation and/or reaction of the compounds above $125{ }^{\circ} \mathrm{C}$. Further investigations were performed to try to identify the degradation products of 4METBZOH and 34METBZOH. Considering the nature of the molecules and the conditions of test bulk, one of the most likely reactions would be hydrolysis of the methoxy groups generating methanol. This hypothesis was not verified as the presence of methanol was not analytically verified in any of the samples in the end of the experiments (Paper III). The results for these two compounds are presented in Figure 21. 

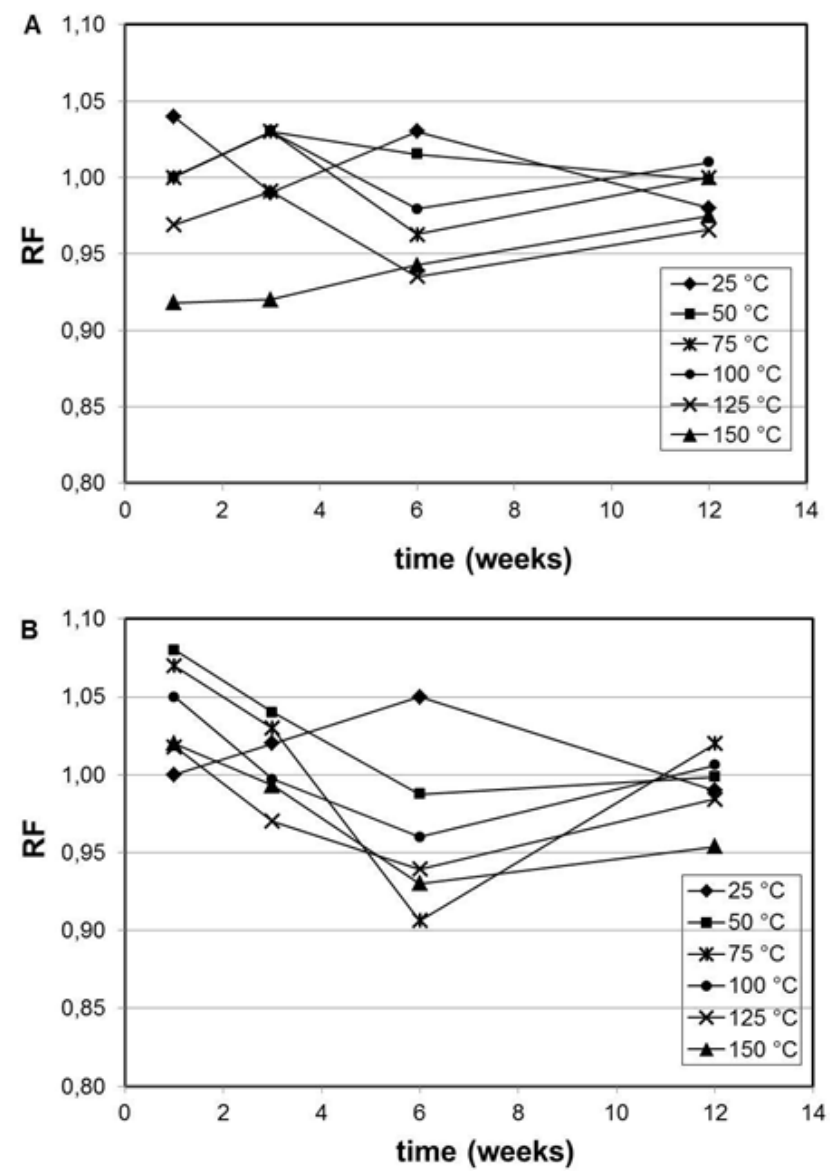

Figure 20. RF of 4-Chlorobenzyl alcohol and 2,6-Dichlorobenzyl alcohol as function of time and temperature of incubation. (A) 4-Chlorobenzyl alcohol, (B) 2,6-Dichlorobenzyl alcohol. Initial experimental $\mathrm{pH} 7.1$, no rock substrate). 
Main results and discussion
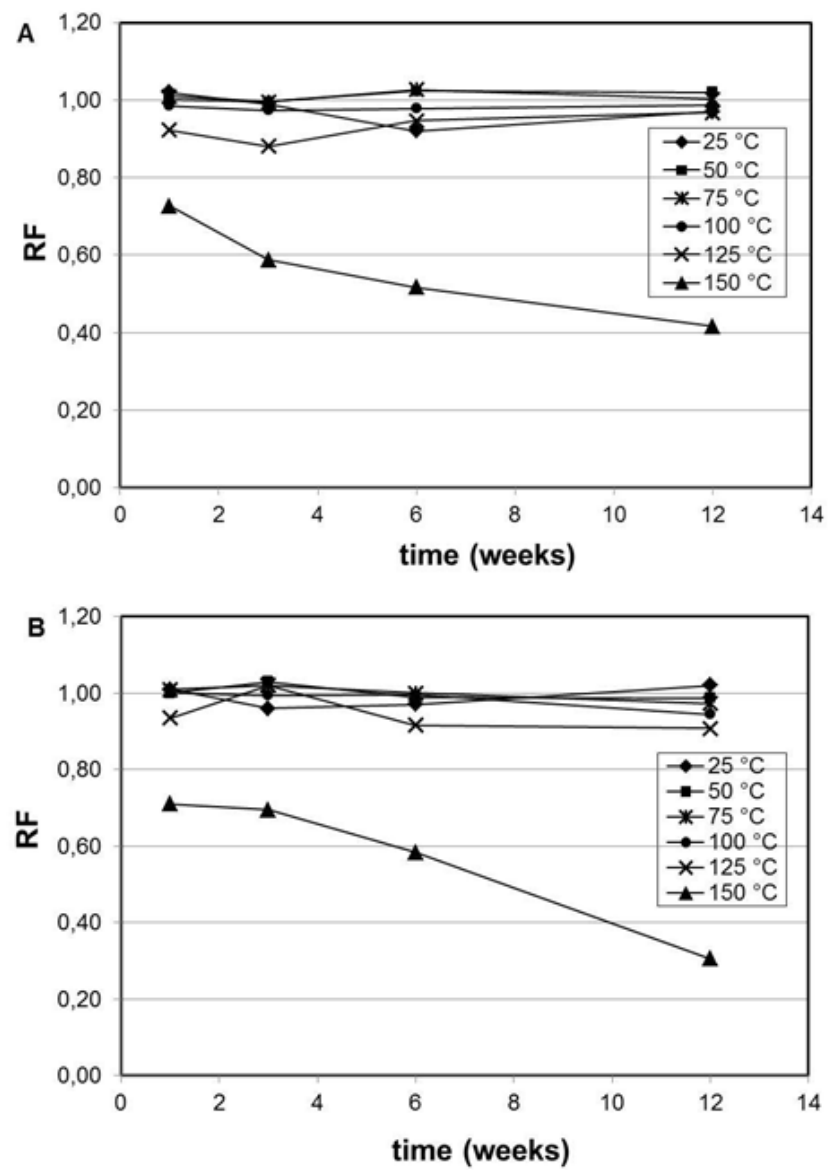

Figure 21. RF of 4-Methoxybenzyl alcohol and 3,4-Dimethoxybenzyl alcohol as function of time and temperature of incubation. (A) 4-Methoxybenzyl alcohol, (B) 3,4-Dimethoxybenzyl alcohol. Initial experimental $\mathrm{pH} 7.1$, no rock substrate).

4-Hydroxybenzyl alcohol (4HYBZOH) is highly stable for 12 weeks at $25{ }^{\circ} \mathrm{C}$ and $50{ }^{\circ} \mathrm{C}$ and degrades at temperatures $\geq 75{ }^{\circ} \mathrm{C}$, see Figure 22. The rate of degradation of $4 \mathrm{HYBZOH}$ is clearly temperature dependant, increasing with the increase of $\mathrm{T}$ (lowest values for $\mathrm{RF}$ are achieved faster as $\mathrm{T}$ increases). The decrease in the RF is not linear at any tested temperature which suggests that the degradation kinetics is concentration dependant. A linear decrease in the RF of the compound should be expected if the order of the reaction kinetics was zero relatively to the 
concentration of 4-HYD-BZA. Further treatment of the experimental data relative to this compound was performed to assess the possibility of its use as a non-conservative active reservoir tracer to retrieve information about temperature in flooded areas of an oil reservoir. We found that a pseudo-first order kinetic model describes the degradation observed for $4 \mathrm{HYBZOH}$ at temperatures $\geq 75^{\circ} \mathrm{C}$.

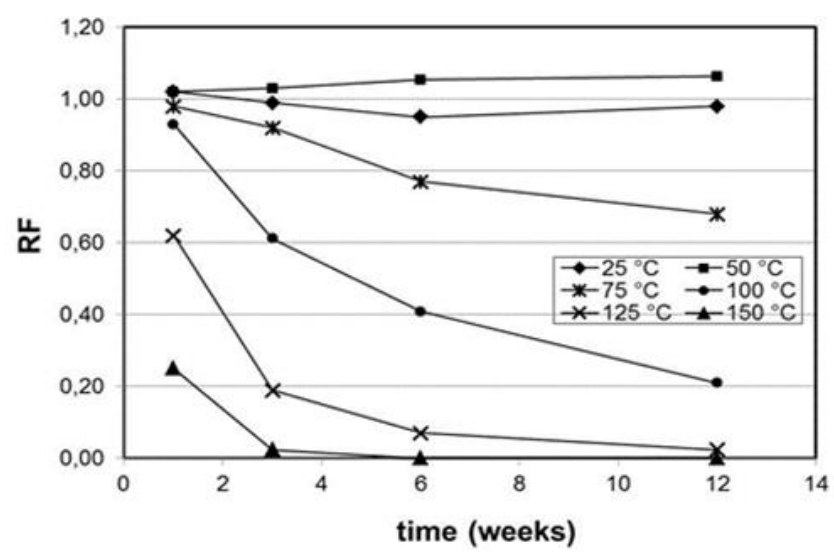

Figure 22. RF of 4-hydroxybenzyl alcohol as function of time and temperature of incubation. Initial experimental $\mathrm{pH} 7.1$, no rock substrate.

A pseudo-first order kinetic model was successfully developed from the experimental data and validated with two additional experiments measured after 2 and 4 weeks of thermal incubation (Paper III). The comparison between the values predicted by the kinetic model and the experimental ones is presented in Figure 23. The apparent preexponential factor of the Arrhenius equation and apparent activation energy were determined and are presented in Eq. 10.

$\ln \left(\frac{C_{A}}{C_{A 0}}\right)=-2,30 \times 10^{07} e^{\frac{-58,8 \times 10^{03}}{R T}} \cdot t$

(Eq. 10)

$\mathrm{C}_{\mathrm{A}}$ is the concentration of 4-HYD-BZA at a given moment in time, $\mathrm{C}_{\mathrm{A} 0}$ is the initial concentration of 4-HYD-BZA and $t$ is the time in weeks. 


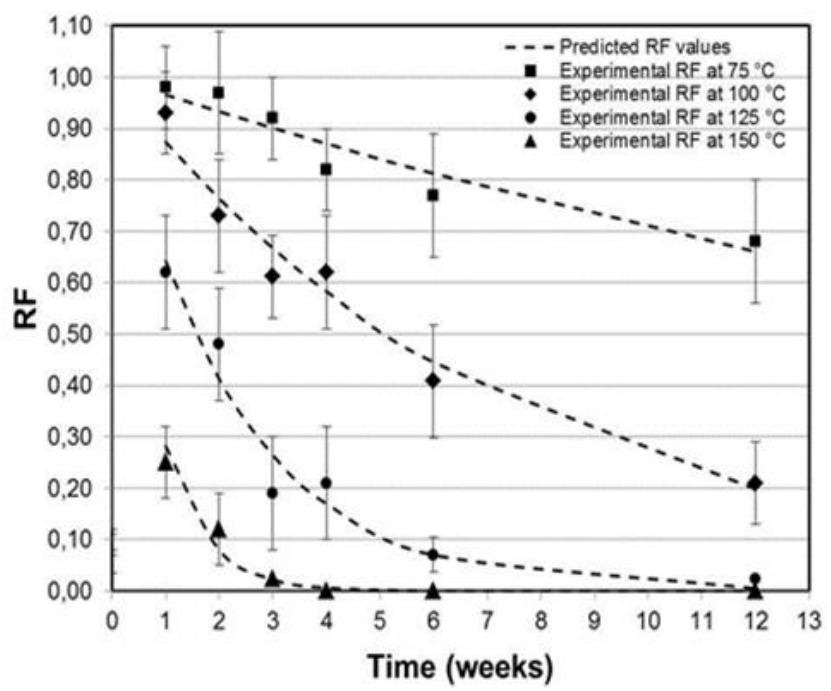

Figure 23. Experimental vs predicted RF values of 4-hydroxybenzyl alcohol between 75 and 150 ${ }^{\circ} \mathrm{C}$. Uncertainty is not depicted for values under the lower analytical threshold $(\mathrm{RF}=0,05)$.

This was determined under the experimental conditions of the present study. Thus, care should be taken to extrapolate to field conditions, particularly because the possible degradation reaction products were not identified (the model is based on a pseudo-first order kinetics based on the concentration of $4 \mathrm{HYBZOH})$. Still, this suggests, however, that $4 \mathrm{HYBZOH}$ could be used to retrieve thermal data from the inter-well region (in combination with a mass conservative tracer) if the degradation observed in the produced waters follows the same trend. This information could be important for many EOR techniques, to detect possible thermal fronts, or provide additional data for the PITT as the values of K are typically temperature dependant (Paper VII).

All the tracer candidates from this family of chemicals were found to be insensitive to the presence of rock materials. Like for pyrazines, the RF values in the experiments with and without rock material were compared and the maximum differences are presented in Figure 24. Even the maximum differences encountered are not clearly outside the analytical uncertainty. Thus, it can be argued that no influence from the rock materials is seen. 
The outcome of this set of experiments on the benzyl alcohols was that 4ClBZOH, 26ClBZOH, 4METBZOH, 34METBZOH were subjected to further tests, while 4HYBZOH was excluded (Paper III).

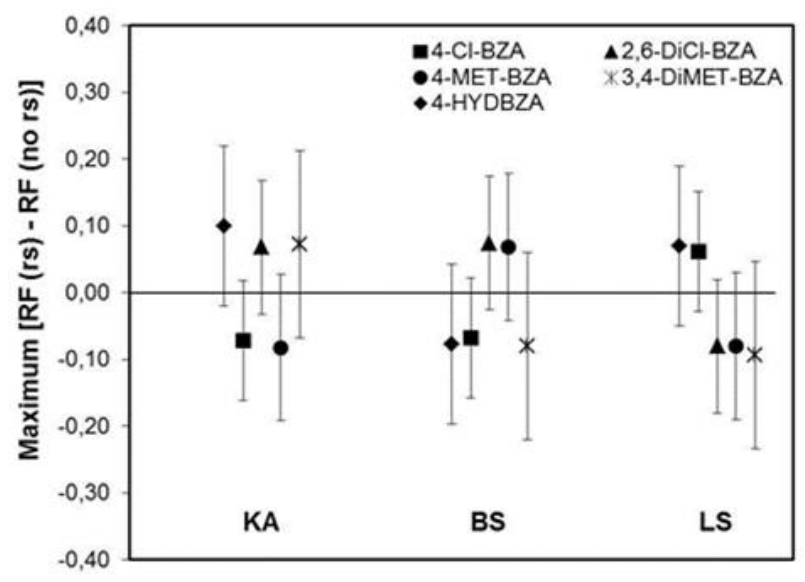

Figure 24. Maximum differences encountered in RF values between experiments with rock substrate (rs) and experiments without rock substrate $\pm 2 \mathrm{x} \sigma$ in RF (t, T). (KA) kaolinite, (BS) Berea sandstone, (LS) limestone, vs predicted RF values of 4-hydroxybenzyl alcohol between 75 and $150{ }^{\circ} \mathrm{C}$. Uncertainty is not depicted for values under the lower analytical threshold $(\mathrm{RF}=$ $0,05)$.

\subsubsection{Stability of pyridines}

Within the group of pyridines evaluated, pyridine itself is the single compound that exhibits the required mass conservation as function of temperature and time of thermal incubation. Results show that PYR is also not affected by the presence of any of the rock materials or by the different $\mathrm{pH}$ conditions tested. PYR was one of the compounds tested further (Paper V), see Figure 25. Thermal and time dependant degradation is observed for 2-hydroxy-6-methylpyridine, that only appears to be stable at $25^{\circ} \mathrm{C}$. Results suggest that $\mathrm{pH}$ has some influence on the degradation rate of 2HY6MPYR (Figure 26). Particularly for shorter incubation periods and high temperatures, an argument can be made that higher initial $\mathrm{pH}$ values reduce the speed of degradation. 

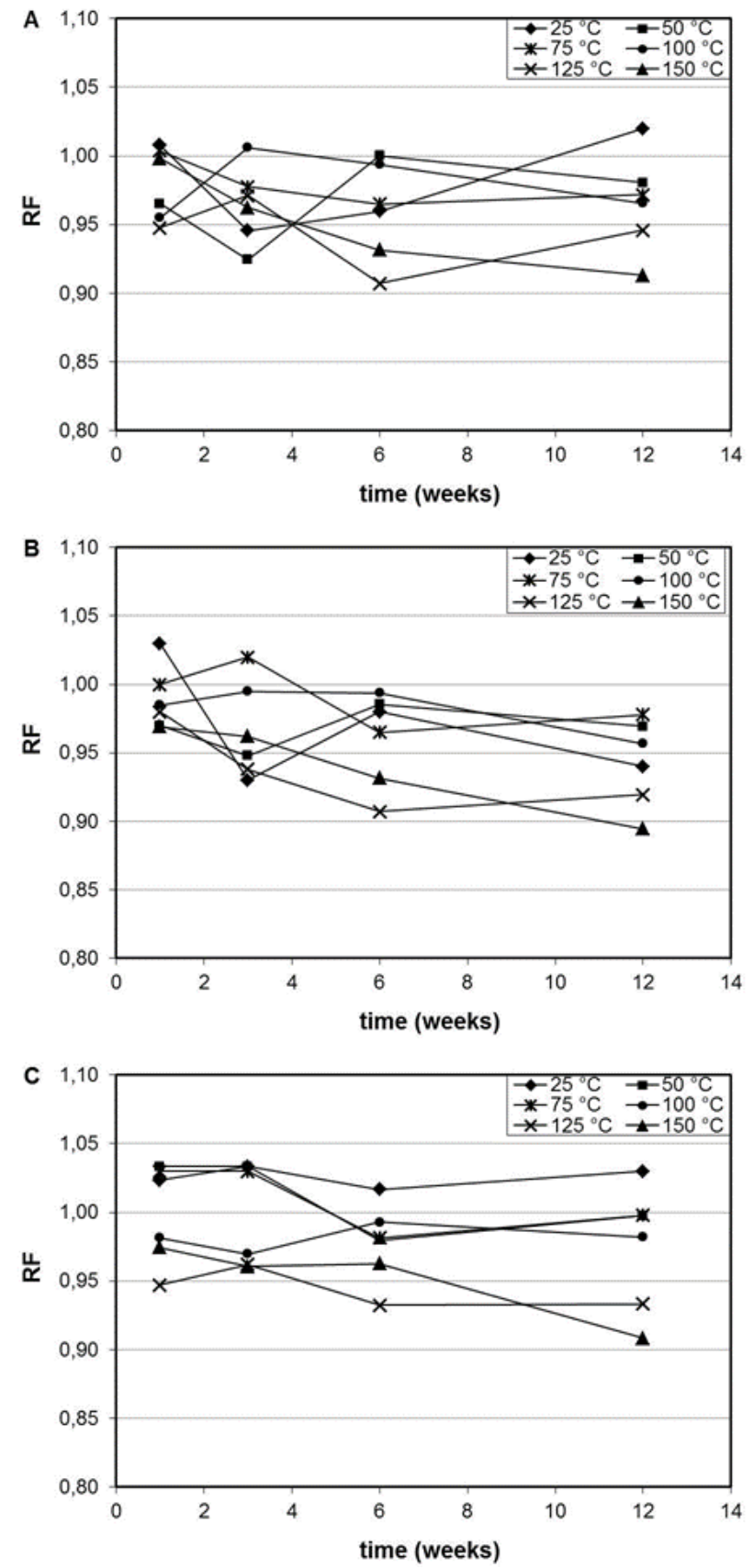

Figure 25. RF of pyridine as function of time and temperature of incubation. (A) Initial $\mathrm{pH} 5.5$; (B) Initial $\mathrm{pH} 7.1$; (C) Initial $\mathrm{pH} 8.0$ and no rock substrate in any of the samples. 

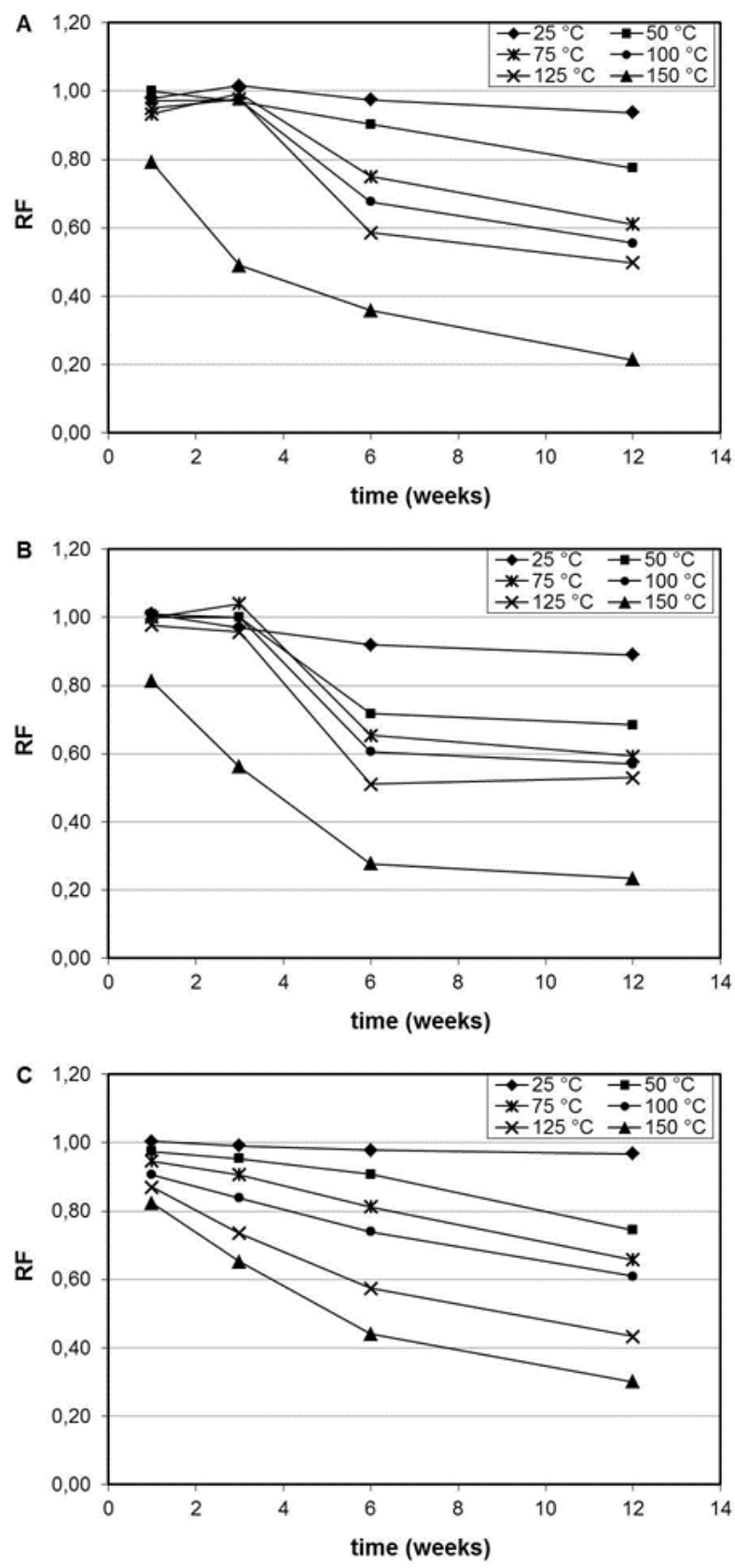

Figure 26. RF of 2-hydroxy-6-methylpyridine as function of time and temperature of incubation. (A) Initial $\mathrm{pH} 5.5$; (B) Initial $\mathrm{pH} 7.1$; (C) Initial $\mathrm{pH} 8.0$ and no rock substrate in any of the samples. 
However, with increasing incubation periods, this protective effect from $\mathrm{pH}$ tends to fade. Long-term mass conservation is a requirement for a PITT tracer. Thus, no further work was done with 2HY6MPYR (Paper V).

Results show that 3-hydroxypyridine (3HYPYR) and 4methoxypyridine (4METPYR) also do not possess the required thermal stability to ensure mass conservation, except at low temperature reservoirs, see Figures 27 and 28.

Like for 2HY6MPYR, higher $\mathrm{pH}$ values seem to reduce the rate of degradation of 3 HYPYR in the earlier phase of the experiments (RF values tend to be higher in the experiments at initial $\mathrm{pH} 8.0$ than at $\mathrm{pH}$ 7.1, which in turn, are higher than at $\mathrm{pH}$ 5.5.). This effect disappears, however, with longer periods of incubation and temperature becomes the dominating factor behind the decrease in the RF values of this compound. 

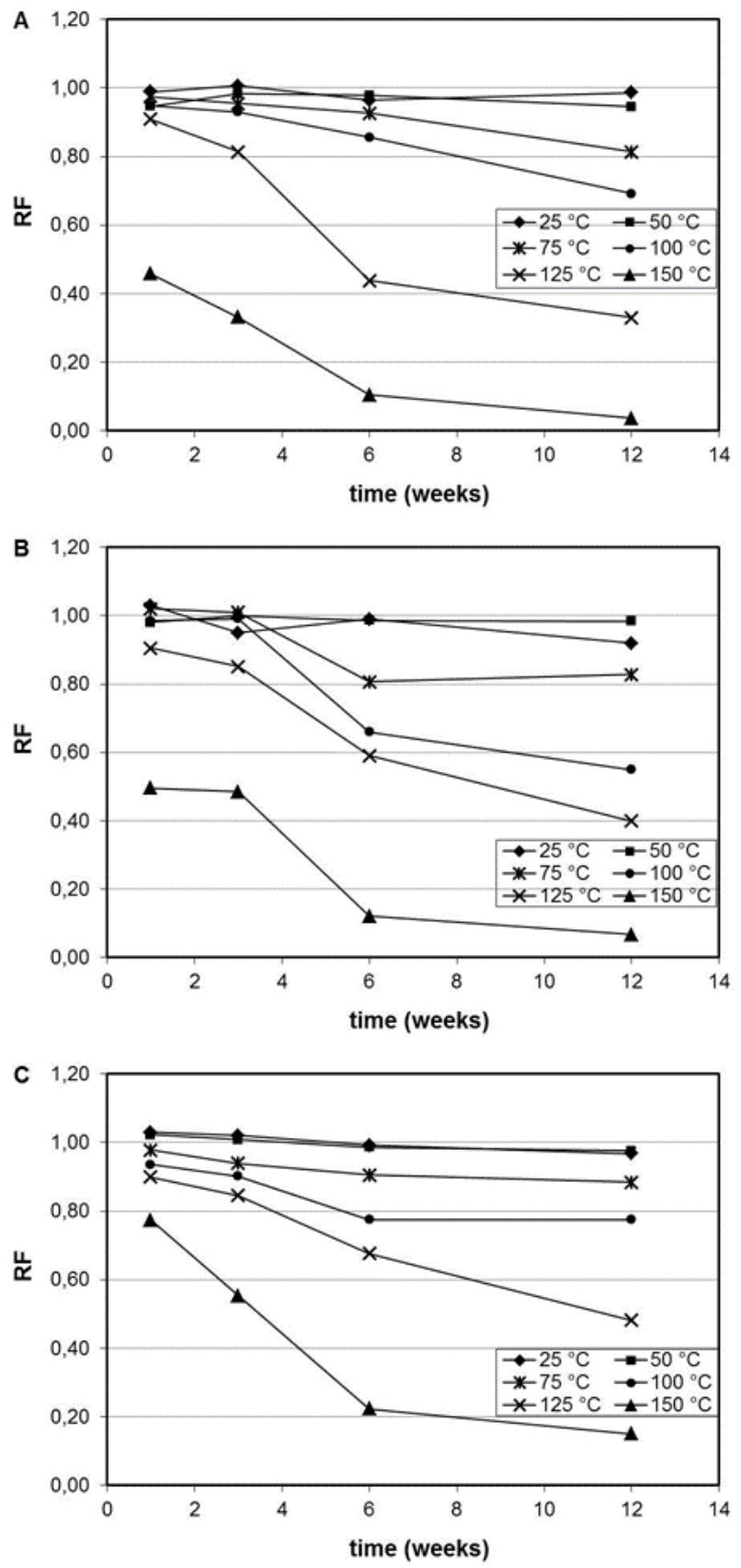

Figure 27. RF of 3-hydroxypyridine as function of time and temperature of incubation. (A) Initial $\mathrm{pH} 5.5$; (B) Initial $\mathrm{pH} 7.1$; (C) Initial $\mathrm{pH} 8.0$ and no rock substrate in any of the samples. 

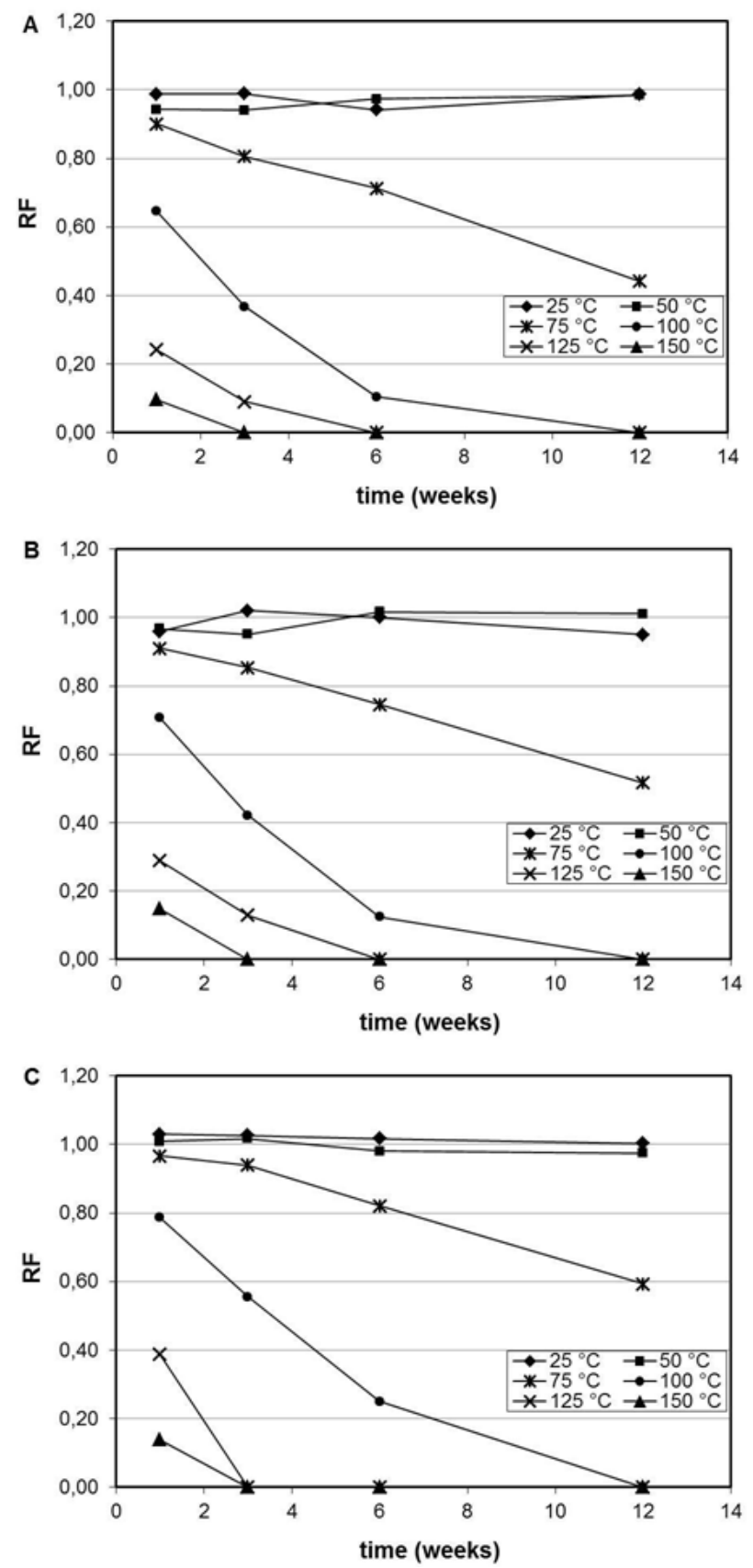

Figure 28. RF of 4-methoxypyridine as function of time and temperature of incubation. (A) Initial $\mathrm{pH} 5.5$; (B) Initial $\mathrm{pH} 7.1$; (C) Initial $\mathrm{pH} 8.0$ and no rock substrate in any of the samples. 
Both 3HYPYR and 4METPYR exhibit significant reductions in the RF values when in contact with kaolinite, as shown in Figure 29, with significant differences encountered between the experiments with and without this mineral. Furthermore, results in Figure 29 for 4METPYR do not appear to show any combined effect of kaolinite and $\mathrm{pH}$. This is not the case for 3HYPYR. The maximum RF differences between 3 HYPYR in the samples with kaolinite and without any rock materials seem to increase towards lower $\mathrm{pH}$. This led to further treatment of the data (presented in figure 30) to assess the full evolution of these differences as function of $\mathrm{pH}$ and temperature.

Excluding the differences observed at $150{ }^{\circ} \mathrm{C}$, the influence of kaolinite on the RF-value of 3-hydroxypyridine (3HYPYR) seems to be independent of temperature. The variation observed at $150{ }^{\circ} \mathrm{C}$ can be explained by the extension of the thermally driven degradation. At all other temperatures, a combined effect between $\mathrm{pH}$ and kaolinite is observed on the presence of 3HYPYR. The lower the $\mathrm{pH}$ of the experiment, the larger is the decrease in the concentration of 3hydroxypyridine in the presence of kaolinite. Different chemical species of 3HYPYR occur at different $\mathrm{pH}$ values. As the $\mathrm{pH}$ becomes lower, the extension of deprotonation of the $\mathrm{OH}$ group increases as well as the protonation oh the nitrogen in the ring of the molecule. Kaolinite has been described both as adsorbent and catalyst for organometallic reactions, thus complex reactions involving the clay and 3HYPYR can take place. In Paper V, the hypothesis of kaolinite acting as a catalyst for a reaction between 3HYPYR and one of the metallic cations present in solution is advanced as it would fit the experimental data. If such reaction occurred with one of the lowest concentration ions, it would stop once it is fully consumed. This would explain the apparent independence from temperature of the phenomena, as adsorption does not describe well the data. 

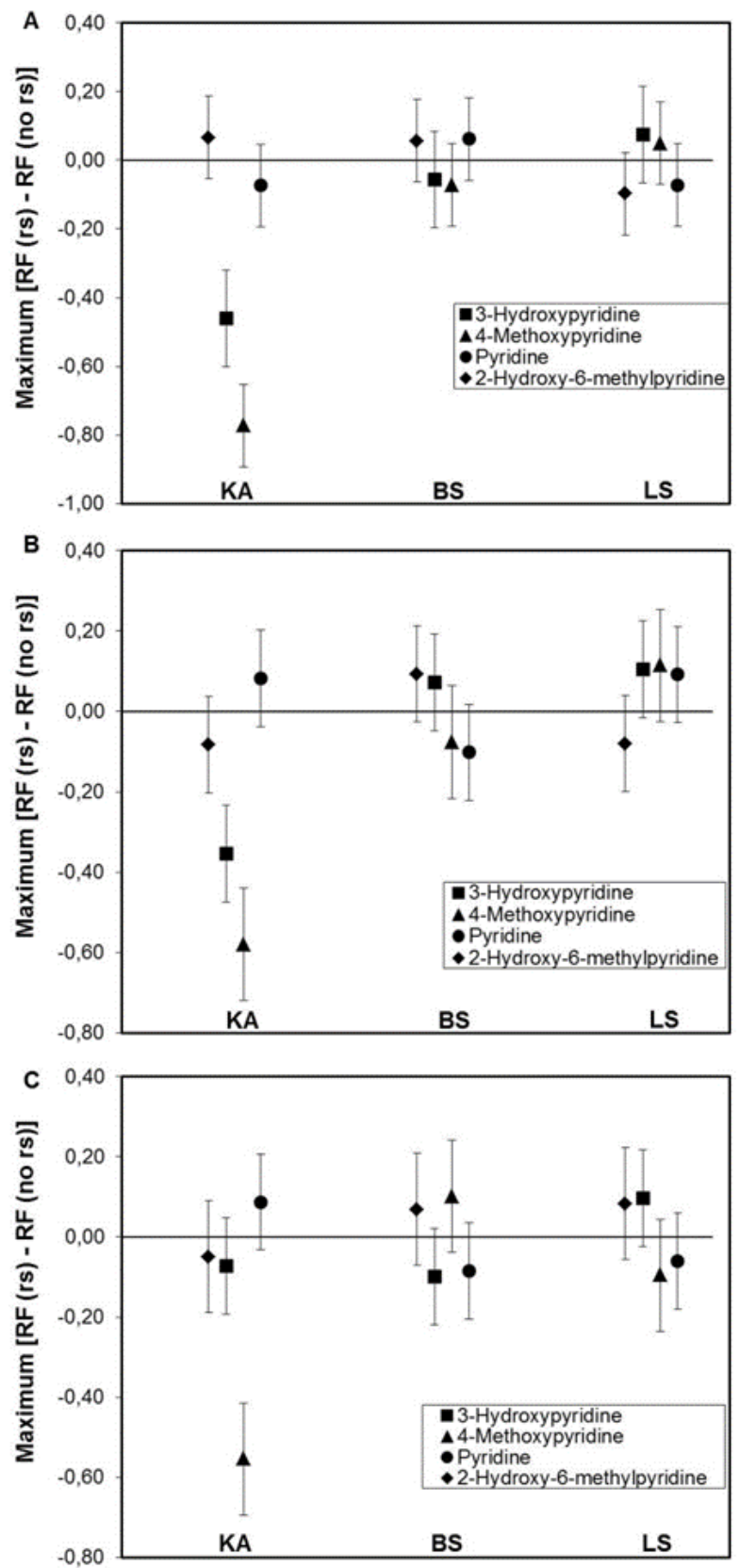

Figure 29. Maximum differences encountered in RF values between experiments with rock substrate (rs) and experiments without rock substrate $\pm 2 \times \sigma$ in RF (t, T). (KA) kaolinite, (BS) Berea sandstone, (LS) limestone. (A) Initial pH 5.5; (B) Initial pH 7.1; (C) Initial pH 8.0. 


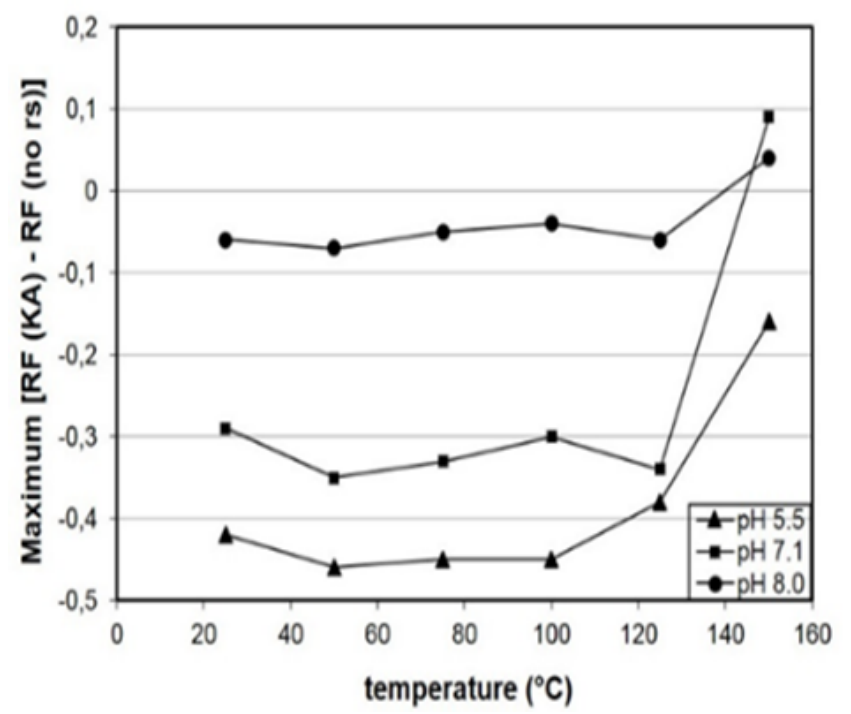

Figure 30. Maximum differences encountered in RF-values of 3-hydroxypyridine between experiments with kaolinite (KA) and experiments without rock substrate (rs) as function of temperature and $\mathrm{pH}$.

Like for HYBZOH in section 3.1.2., a pseudo-first order kinetic model describes well the decrease in RF values of 4METPYR. The same analysis and additional experiments were performed. Equation 11 is the mathematical expression of the model developed with the apparent preexponential factor of the Arrhenius equation and apparent energy of activation.

$\ln \left(\frac{C_{A}}{C_{A 0}}\right)=-1,86 X 10^{7} e^{\frac{-56,4 E^{3}}{R T}} \cdot t$

\subsubsection{Stability of linear diols}

1,2-Hexanediol (12HEXDIOL) and 1,6-hexanediol (16HEXDIOL) exhibit very low stability and were early in the study deemed unsuitable for use in any application where mass-conservation is required (Papers I and II), see Figures 31 and 32. 


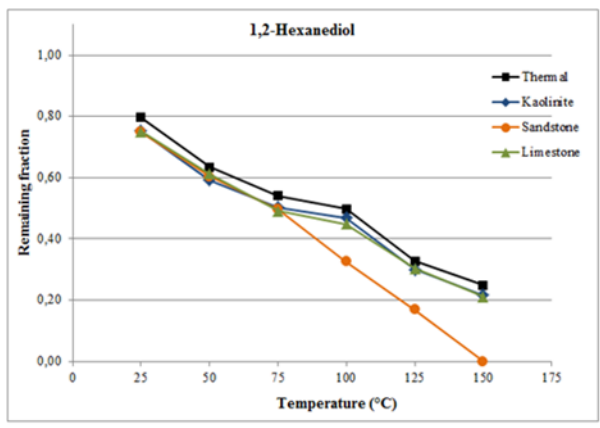

A

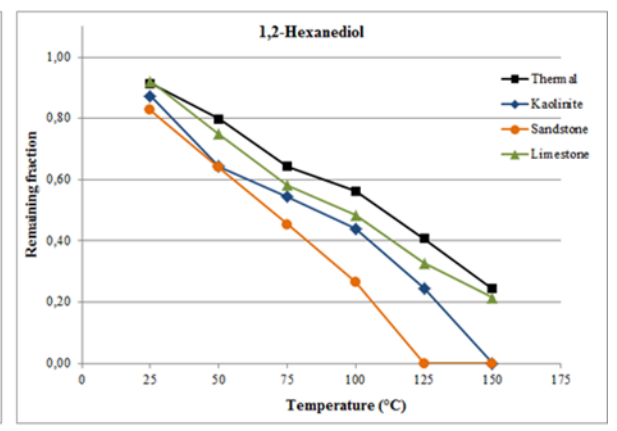

B

Figure 31. Remaining fraction of 1,2-hexanediol as a function of temperature after 12 weeks with and without rock substrates (A: initial $\mathrm{pH} 5.5$; $\mathrm{B}$ : initial $\mathrm{pH}$ 8.0).

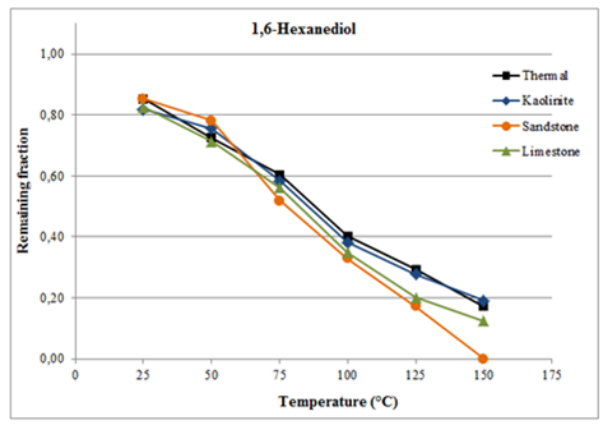

A

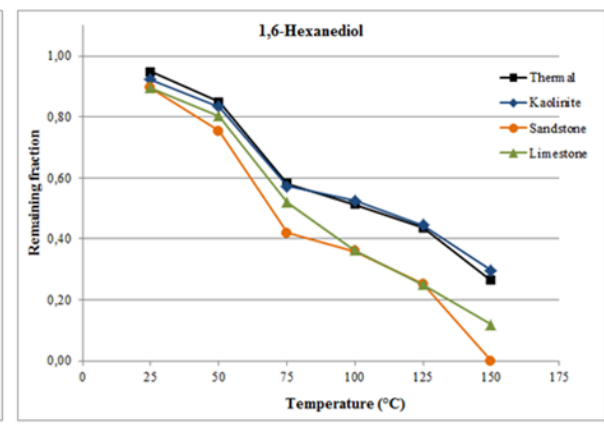

B

Figure 32. Remaining fraction of 1,6-hexanediol as a function of temperature after 12 weeks with and without rock substrates (A: initial $\mathrm{pH}$ 5.5; $\mathrm{B}$ : initial $\mathrm{pH} 8.0$ ).

Additionally, 12HEXDIOL appears to be impacted by the presence of rock materials. This is most likely due to the polar characteristics of the molecule. The presence of the $2 \mathrm{OH}$-groups leads to un-uniform charge distribution through the structure, inducing a negative dipole on the side of the $\mathrm{OH}$ groups and a positive dipole on the opposing end of the molecule (a surfactant-like behaviour). These dipolar moments likely lead to some adsorption of this compound onto the rock surface. Even with a preliminary analysis of the results (Papers I and II), the use of linear-chain alcohols as tracers in inter-well applications seems to be highly risky. Thus, no further work was done with these diols. 


\subsubsection{Summary of the stability experiments}

These stability experiments allowed to identify seven of the initial sixteen oil/water partitioning tracer candidates as suitable for further steps of the qualification process. Pyridine, 2,3-dimethylpyrazine, 2,6dimethylpyrazine, 4-chlorobenzyl alcohol and 2,6-dichlorobenzyl alcohol are fully stable under all tested temperatures for 12 weeks. The molecules show no interaction with any of the rock materials tested (Berea sandstone, kaolinite, and limestone) and are not affected by the $\mathrm{pH}$ conditions $(5,5$ - 8,0). 4-Methoxybenzyl alcohol and 3,4dimethoxybenzyl alcohol also share these characteristics, but with thermal stability limited to $125^{\circ} \mathrm{C}$ and below. This is still a sufficiently high stability to allow these two compounds to be used in most oilfields.

\subsection{Development of an analytical method for real field samples}

The target analytes for the analysis method developed here for real field samples were the oil/water partitioning tracer candidates qualified in section 3.1. It was necessary to identify and quantify these compounds in produced waters from oilfield in concentrations of low $\mu \mathrm{g} / \mathrm{L}$, or, ideally, even lower. The lowest possible LOQs are desirable to increase the accuracy of the recorded tracer production curves while simultaneously reducing the amount of chemicals used in the test (AlAbbad et al., 2018; Viig et al., 2013). This is particularly an important factor in offshore operations due to HSE and logistic issues and large well distances.

In sections 2.3.1. and 2.3.2. analytical techniques based on UPLC-UV for analysis of the tracer candidates were presented. However, UV detection does not allow to reach the LOQs needed for field samples. Thus, UPLC-MS was considered. The benzyl alcohols (like most alcohols) are not easily analysable by UPLC-MS. The instrument available was equipped with two possible ion sources: electrospray ionisation (ESI) and atmospheric pressure chemical ionisation (APCI). 
ESI can be operated in positive or negative mode and consists basically of creating conditions to protonate or de-protonate the analytes. It is also the preferred ion source for routine analysis due to its higher reproducibility. Benzyl alcohols have very high $\mathrm{pKa}$ values, thus are not proton donors or acceptors. These four compounds would not be possible to analyse using ESI.

APCI is a soft ionisation technique where the primary ions are produced on a solvent spray through ion-molecule reactions at atmospheric pressure triggered by an electrical discharge provided by the instrument (Thomson, 1998). The ions generated by a given molecule in APCI can vary from sample to sample in both relative abundances and species (de Hoffmann and Stroobant, 2007), making the use of APCI more appropriate for molecules of higher molecular masses and hard/difficult to ionise (steroids, for example).

The analysis by GC of compounds from the same "chemical families" as the stable 7 PITT tracer candidates has been reported, as for example, in the works by (Bakaikina et al., 2018), (Frank et al., 2004) and (Câmara et al., 2006). Additionally, the difference in volatilities of the target analytes (see Table 1) suggests they should be easily separated in a low polarity column. GC-MS was then selected as the instrumental technique to be used.

Three ionisation techniques were available for this method: electron impact ionisation $(\mathrm{EI})$, positive chemical ionisation $(+\mathrm{CI})$ and negative chemical ionisation (-CI). EI is a hard ionisation method where the gas current entering the MS is bombarded with an electron beam with an energy of $70 \mathrm{eV}$. Electrons are ejected from the gas molecules and positive ions are generated. The molecules are also typically fragmented generating additional ions. This method has high qualitative value, because a given molecule will always generate the same ions with the same relative abundancies (de Hoffmann and Stroobant, 2007). Thus, ion spectra that are nowadays available for several millions of compounds are widely used in qualitative analysis for molecular masses below about 1000 Dalton (D). Positive ions are then separated and detected in the MS. 
CI is a soft ionisation method where the ions of the analytes are produced in ion-molecule reactions resulting from the interactions of a reactant gas, previously ionised by EI, with the gas molecules entering the MS (de Hoffmann and Stroobant, 2007). The analytes should have highly electronegative substituents for efficient generation of negative ions and the generation of positive ions will depend on the existence of sufficiently strong dipoles that will accept protons.

Preliminary tests were made with our tracer candidates and it was concluded that EI is the most efficient technique for the scope of the present work. It yielded more qualitative information and more intense ions.

Before analysis by GC-MS, production waters from oilfields must be submitted to a cleaning/concentration step that allows to achieve the desired LOQs and reduces/eliminates interferences that might be present. Solid phase-extraction (SPE) often followed by phase-reconstitution or derivatisation (Muller and Seubert, 2012) is the most used method for sample cleaning/enrichment. However, SPE is labour-intensive, time consuming. The high number of samples to be analysed during the present laboratory tests would require the use of large amounts of solvents, which is not desirable. In fact, the sample preparation step can be defined as the "bottleneck" of the analysis (Boyaci et al., 2016; Muller and Seubert, 2014).

There was previous evidence (section 2.3.1.) that the SPME method would extract many of the compounds investigated in the present work from an aqueous phase. Thus, SPME was tested as sample cleaning/concentration technique for the method to be developed. SPME is also a mature technology, commercially available with many different adsorbent phases, allowing for a routine large output of analysis.

By this work, SPME was introduced for the first time, to the best of the author's knowledge, as sample pre-treatment technique for analysis of produced waters from oilfields (Paper VI). Preliminary tests were performed to select the most appropriate SPME fibre. Results (not shown here but available in Paper VI) proved that the 
divinylbenzene/carboxen/polydimethylsiloxane (DVB/CAR/PDMS) $(50 / 30 \mu \mathrm{m})$ fibre is the most effective type of fibre for simultaneous extraction of the analytes (the stable PITT tracer candidates).

The background noise obtained from real production water required mass separation detection to be performed using tandem MS in selected reaction monitoring (SRM) mode. The instrument used is based on a triple quadrupole. With this configuration and using tandem MS (also known as MS/MS), the desired ions are selected in the first quadrupole, fragmented in the second quadrupole by collision with argon, and the generated ions filtered in the third quadrupole and sent to the mass analyser. The collision energies were optimised for each of the analytes, three transitions were used for identification of the analytes and the transition that yielded the most intense signals used for quantification (Paper VI).

The conditions of SPME extraction were optimised. Direct immersion (DI) would yield the best signals for standards in deionised water, however even short periods (above 6 minutes) would introduce interferences when extracting the analytes from real field samples. A sequential DI-HS-SPME step was developed by using the salting-out effect and heating of the samples after initial $\mathrm{pH}$ adjustment to 9,0 (to ensure that pyridine is unprotonated). The response peak areas were maximised as function of the extraction conditions. Using $5 \mathrm{~mL}$ of sample, the optimised extraction procedure consisted of addition of 1.8 $\mathrm{g}$ of $\mathrm{NaCl}$, constant stirring, 5 minutes of DI-SPME followed by 15 minutes of HS-SPME at $70{ }^{\circ} \mathrm{C}$ using a DVB/CAR/PDMS $(50 / 30 \mu \mathrm{m})$ fibre. Figure 33. (from Paper VI) shows the peak areas as function of the extraction conditions.

Results in Figure 33 were obtained from real production water from the Ekofisk field $\mathrm{M}$ spiked with $10 \mu \mathrm{g} / \mathrm{L}$ of each of the PITT tracer candidates. The response areas are very similar at $70{ }^{\circ} \mathrm{C}$ and $80{ }^{\circ} \mathrm{C}$, however results at $70{ }^{\circ} \mathrm{C}$ exhibit a systematic trend above the ones at 80 ${ }^{\circ} \mathrm{C}$. 


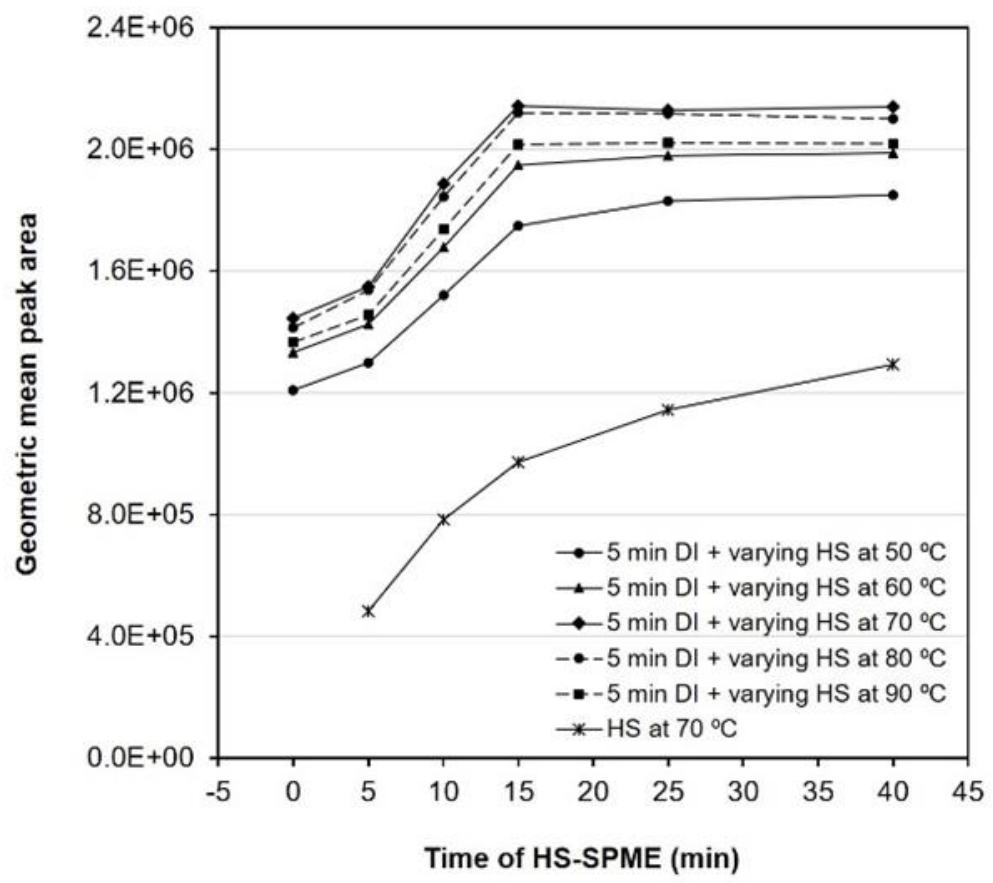

Figure 33. Geometric mean response area of the target compounds as function of the time and temperature of HS-SPME extraction after a fixed DI-SPME period of 5 minutes.

SPME is not an exhaustive extraction technique and the equilibrium of the system was achieved after a relatively short period of 20 minutes ( 5 minutes of DI +15 minutes of HS) of extraction (except when extraction is done at $50{ }^{\circ} \mathrm{C}$ ). This is most likely due to a combined effect of temperature and the that the initial step is DI-SPME. Initiating the sequential extraction procedure with DI maximizes the mass transferring gradient between the bulk of the sample and the SPME fibre, thus maximizing the rate of adsorption. Increasing temperature will increase the volatility of the analytes and thereby promote a fastest transfer to the headspace once the equilibrium between headspace and the bulk of the sample is disturbed by the HS-SPME extraction. It should be noted that this is true until an upper temperature value. The results show that the efficiency of the SPME extraction decreases at a temperature of $90{ }^{\circ} \mathrm{C}$. This can be explained by the reduction of the adsorption capacity of the 
SPME fibre as consequence of an excessively high temperature of operation which promotes some desorption (Paper VI).

Timed data acquisition was used in combination with the SRM mode for the tandem MS of the analytes. This allows to clean up the obtained chromatogram further, increasing the signal to noise ratio $(\mathrm{S} / \mathrm{N})$, thus allowing lower LOQs to be reached. Figure 34 displays a reconstructed SRM chromatogram obtained from a sample of production water from the Ekofisk field $\mathrm{M}$ spiked with the tracers at a concentration of $1 \mu \mathrm{g} / \mathrm{L}$ and extracted with the optimized sequential DI-HS-SPME procedure.

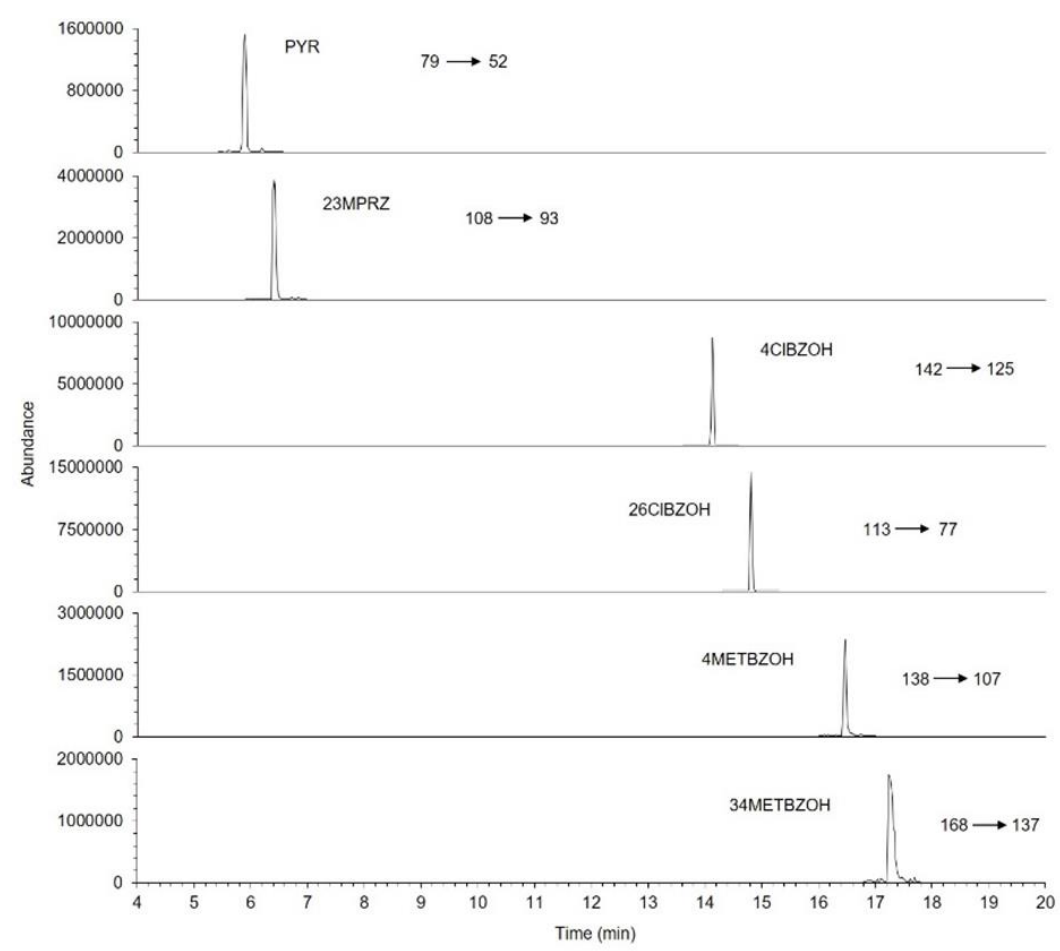

Figure 34. Reconstructed SRM chromatogram of produced water from Ekofisk M spiked at $1 \mu \mathrm{g}$ $\mathrm{L}^{-1}$ with the transitions used for quantification of the PITT tracer candidates indicated.

The linearity, precision, accuracy, and recovery of the DI-HS-GCMS/MS method were evaluated following the guidelines from (Eurachem, 2014). The limits of quantification (LOQ) and limits of detection (LOD) were calculated as the concentration of the compounds 
originating a signal to noise ratio $(\mathrm{S} / \mathrm{N})$ of 10 and 3, respectively, by applying the optimized analytical method to real samples spiked at varying low concentrations. It was verified that the concentrations of the analytes which originate $\mathrm{S} / \mathrm{N} \geq 10$ are systematically lower than the lower linear concentration threshold. LOQ and LOD values are indicated in Table 2. Because the present method is conceived for an application where the processing of many samples is required, the use of the lower limits of linearity as LOQ is recommended for systematic analysis (Paper VI).

LOQs, linear range, intra-day precision $(\mathrm{n}=7)$ and inter-day $(\mathrm{n}=5)$ precision at three different concentration levels $(0.5,2.5$, and $10 \mu \mathrm{g} / \mathrm{L})$ are presented in Table 9.

Table 9 - Linearity, limits of quantification and precision of the DI-HS-SPME-GC-MS/MS evaluated at three concentrations.

\begin{tabular}{|c|c|c|c|c|c|c|c|c|c|}
\hline \multirow{3}{*}{ Tracers } & \multirow{2}{*}{\multicolumn{2}{|c|}{ Linearity }} & \multirow[b]{3}{*}{$\mathrm{LoQ}^{\mathrm{a}}$} & \multicolumn{6}{|c|}{ Precision (\% RSD) } \\
\hline & & & & \multicolumn{3}{|c|}{$\begin{array}{l}\text { Intra-day } \\
\text { precision }\end{array}$} & \multicolumn{3}{|c|}{$\begin{array}{l}\text { Inter-day } \\
\text { precision }\end{array}$} \\
\hline & Range & $\mathrm{R}^{2}$ & & $0.50^{\mathrm{a}}$ & $2.5^{\mathrm{a}}$ & $10^{\mathrm{a}}$ & $0.50^{\mathrm{a}}$ & $2.5^{\mathrm{a}}$ & $10^{\mathrm{a}}$ \\
\hline PYR & $\begin{array}{c}0.50- \\
10\end{array}$ & 0.9995 & 0.35 & 7.9 & 6.6 & 6.2 & 9.4 & 7.4 & 7.3 \\
\hline 23MPRZ & $\begin{array}{c}0.30- \\
50\end{array}$ & 0.9970 & 0.20 & 4.7 & 5.3 & 4.2 & 7.1 & 7.2 & 5.3 \\
\hline $4 \mathrm{ClBZOH}$ & $\begin{array}{c}0.10- \\
20\end{array}$ & 0.9962 & 0.08 & 8.2 & 7.0 & 4.7 & 10 & 8,5 & 5.7 \\
\hline $26 \mathrm{ClBZOH}$ & $\begin{array}{c}0.10- \\
20\end{array}$ & 0.9971 & 0.10 & 6.2 & 7.1 & 4.2 & 8.1 & 7.8 & 5.6 \\
\hline 4METBZOH & $\begin{array}{c}0.40- \\
25\end{array}$ & 0.9974 & 0.25 & 9.4 & 7.4 & 5.9 & 12 & 9.1 & 5.4 \\
\hline 26METBZOH & $\begin{array}{c}0.40- \\
25\end{array}$ & 0.9984 & 0.25 & 10 & 6.4 & 3.9 & 12 & 11 & 7.5 \\
\hline
\end{tabular}

8 different produced water samples from the NCS (Snorre A, Snorre B, Ekofisk M, Gullfaks C, Heidrun A, Eldfisk A, Eldfisk S and Vigdis B) 
were analysed with the method developed to ensure that they were free from background contamination of the PITT tracer candidates. None of the tracer molecules were detected in any of the waters.

The recovery of the method was evaluated by spiking the real produced water samples at three levels of concentration $(0.50,2.50$ and $10 \mu \mathrm{g} \mathrm{L}-$ 1), representative of low, medium, and high concentrations of the analytes. The results show a good performance of the method developed with all the recovery values between $85 \%$ - $115 \%$ and RSDs systematically $\leq 13 \%$. The lowest systematic recoveries were observed for the methoxybenzyl alcohols. This is mostly likely due to their low volatility combined with the fact that the dominant period of SPME extraction is performed on HS mode relatively to the time of DI mode (15 vs 5 minutes). Of all the tested compounds, the measured responses for $4 \mathrm{METBZOH}$ and $34 \mathrm{METBZOH}$ showed the largest difference relatively to HS-SPME (see Figure 33) after 30 minutes of extraction. This suggests that, although 5 minutes of DI enhances the analytical response of the system, is not sufficient time for as fast equilibrium to be reached in the adsorption system (water $\rightarrow$ headspace $\rightarrow$ SPME fibre) for $4 \mathrm{METBZOH}$ and 34METBZOH. However, in summary, results show that the method is suitable for analysis of the oil/water tracer candidates in the present study in produced water with a high sample output capacity (Paper VI).

\subsection{Evaluation of the K-value of the stable PITT tracer candidates}

A series of experiments described in section 2.3.5. were performed to characterise the oil/water partitioning behaviour of PYR, 23MPRZ, 26MPRZ, 3METBZOH, 34METBOZOH, 4ClBZOH and 26ClBZOHas function of temperature, hydrocarbon composition and ion composition of the aqueous phase.

Results show real partitioning (molecular bulk dissolution) of the compounds between the hydrocarbon and the aqueous phase. No 
influence of $\mathrm{pH}$ within the range evaluated (5 - 9) was seen for all compounds (Paper VII), except for pyridine that exhibits K-values with $\mathrm{pH}$ dependency. This validates the assumption that pyridine could be used to obtain real $\mathrm{pH}$ values from the inter-well region if used together with non-pH dependant oil/water partitioning tracer (Paper V). Additionally, the presence of pyridine was detected in produced waters in the present study (Paper VI), further validating the assumptions behind its choosing as candidate.

Any possible surfactant-like behaviour of the PITT tracer candidates could be discarded (Paper VII) since real solutions in both the hydrocarbon and the aqueous phases in contact were confirmed.

The K-values of the PITT tracer candidates as function of the hydrocarbon composition, salinity of the aqueous phase, temperature, and initial phase in which they were dissolved are presented hereafter in Figures $35-41$. $\mathrm{NaCl}$ was used to increase the salt content (ion strength) of the aqueous phase and the hydrocarbon phase in the initial tests was composed of $20 \mathrm{vol} \%$ toluene and varying fractions of octanol $(0 \%-20$ vol\%) and iso-octane $(60 \%-80 \mathrm{vol} \%)$ to vary aromatic/aliphatic composition and polarity. The dashed lines in the figures represent the $\mathrm{K}$-values obtained under the same experimental conditions when the compound is initially dissolved in the hydrocarbon phase. The other values were obtained with the compounds dissolved initially in the aqueous phase.

Results show, in general, that increasing salinity, temperature and amount of octanol in the hydrocarbon phase (higher polarity and lipophilicity) cause an increase in the values of K. Notable exceptions to this were observed for temperature in the cases of $4 \mathrm{ClBZOH}$ and $4 \mathrm{METBZOH}$, and salinity in the case of $26 \mathrm{ClBZOH}$ (Paper VII).

Higher temperature leads to higher K-values due to four fundamental factors: i) higher temperatures mean more energy in the system to disturb the hydrogen bonds between the tracer candidates and water; ii) increased volatility of the non-ionic molecules in aqueous solution making them more prone to leave the aqueous phase; iii) higher 
diffusivity of the molecules across the hydrocarbon/water interface; iv) reduction of the di-electric constant of water that leads to fewer and weaker dipole interactions with non-ionic solutes (Paper VII).

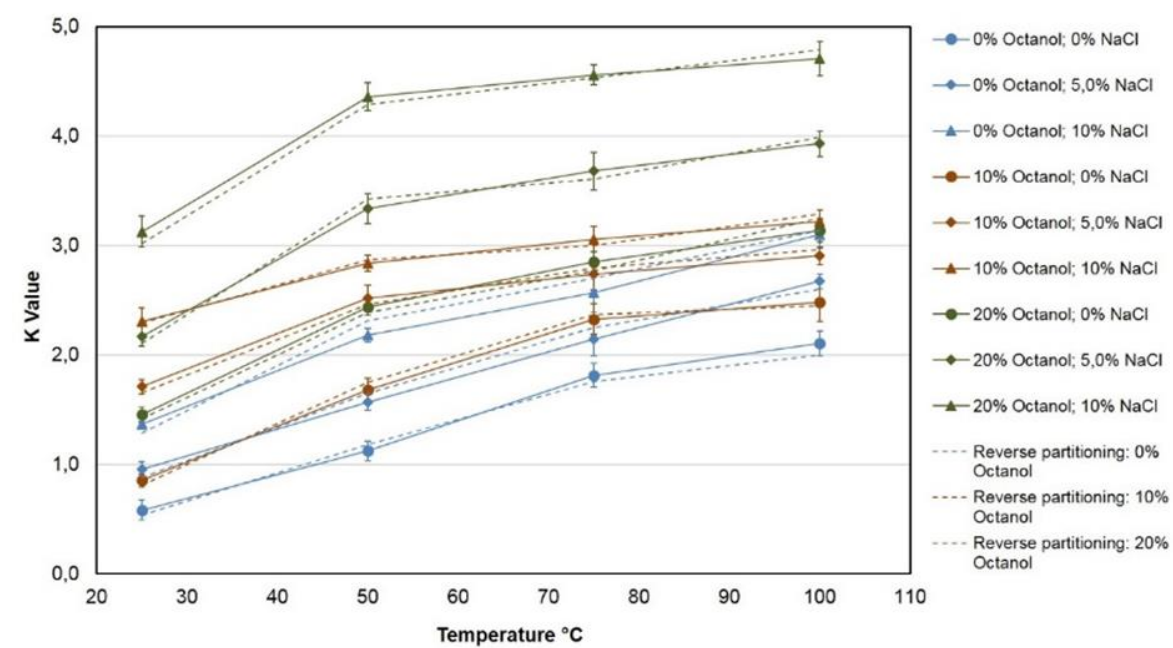

Figure 35. K-values of pyridine $\pm \sigma(\mathrm{N}=3)$ as function of temperature, composition of the hydrocarbon phase and salinity.

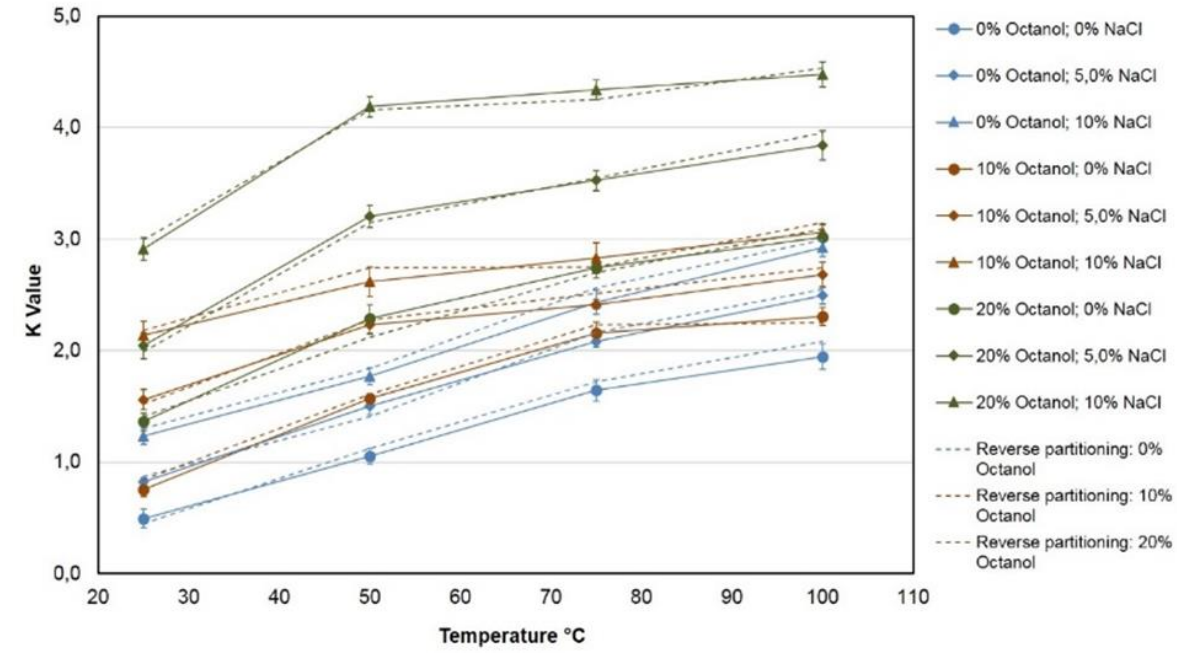

Figure 36. K-values of 2,3-dimethylpyrazine $\pm \sigma(\mathrm{N}=3)$ as function of temperature, composition of the hydrocarbon phase and salinity. 


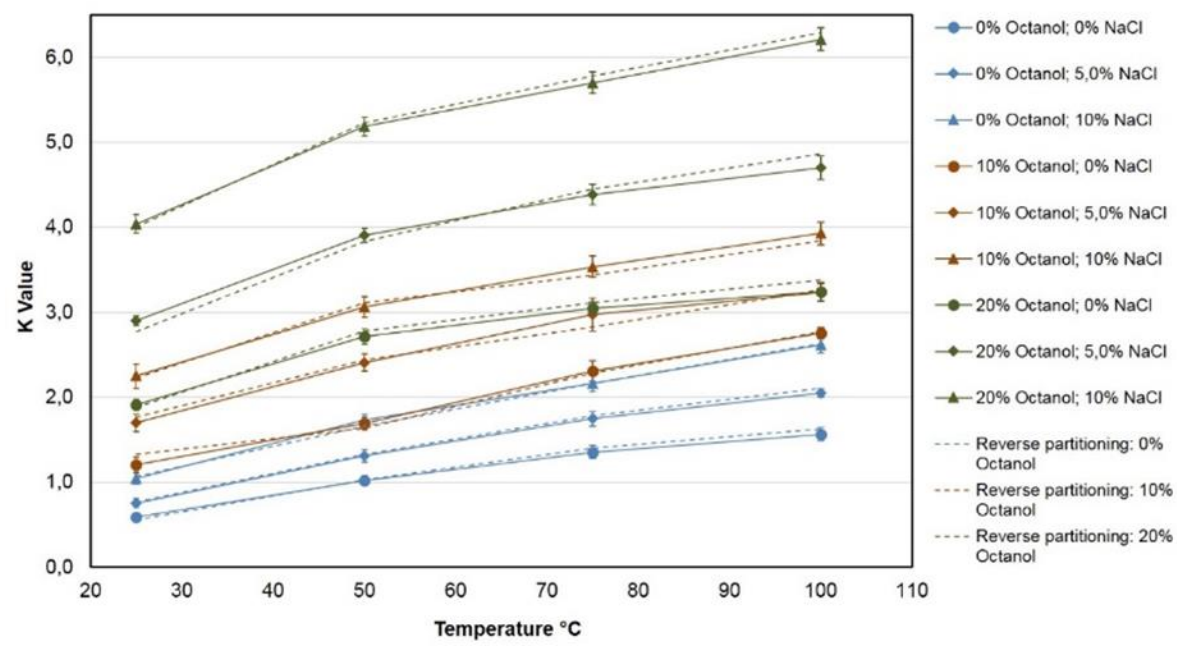

Figure 37. K-values of 2,6-dimethylpyrazine $\pm \sigma(\mathrm{N}=3)$ as function of temperature, composition of the hydrocarbon phase and salinity.

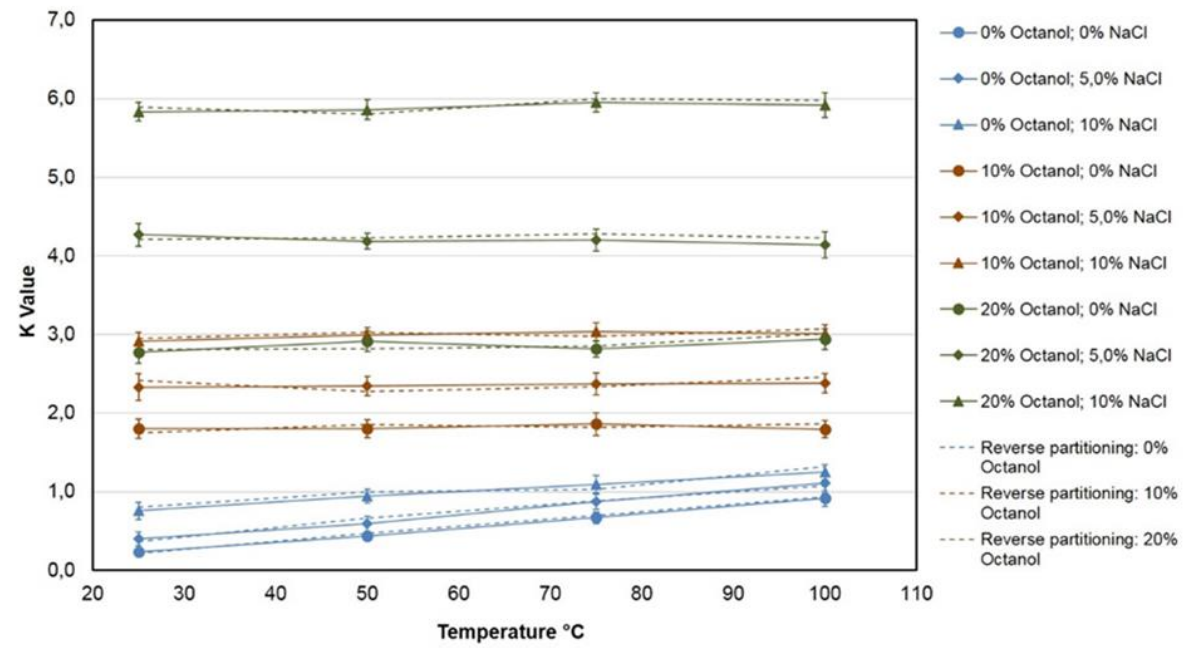

Figure 38. K-values of 4-methoxybenzyl alcohol $\pm \sigma(\mathrm{N}=3)$ as function of temperature, composition of the hydrocarbon phase and salinity. 


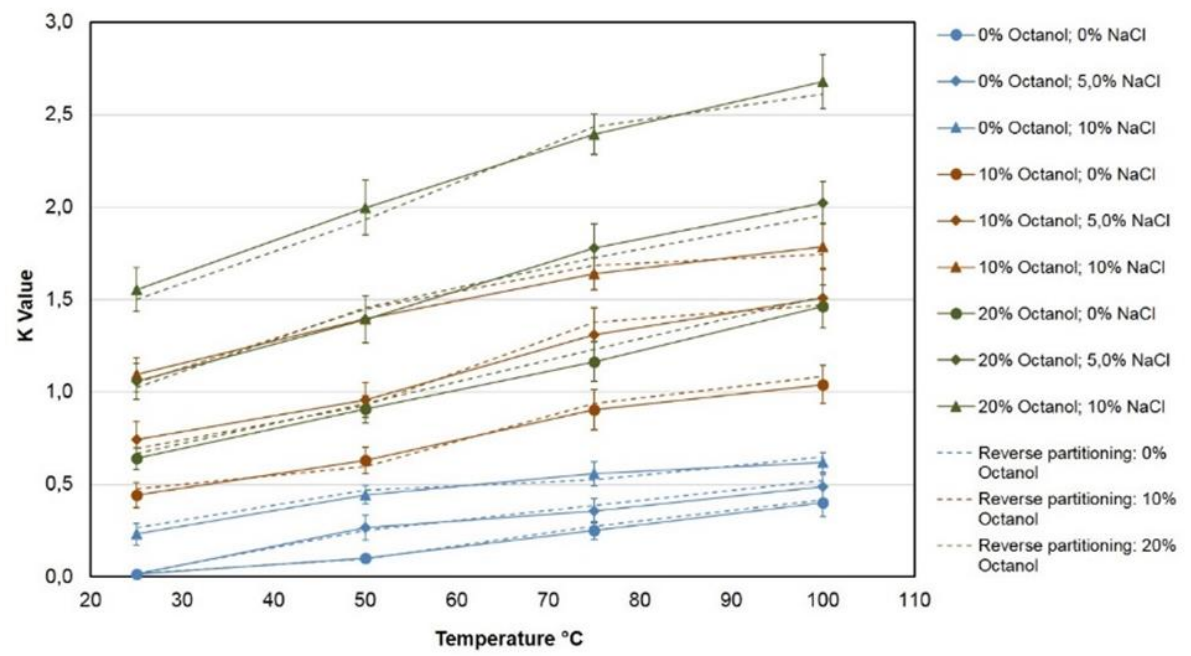

Figure 39. K-values of 3,4-dimethoxybenzyl alcohol $\pm \sigma(\mathrm{N}=3)$ as function of temperature, composition of the hydrocarbon phase and salinity.

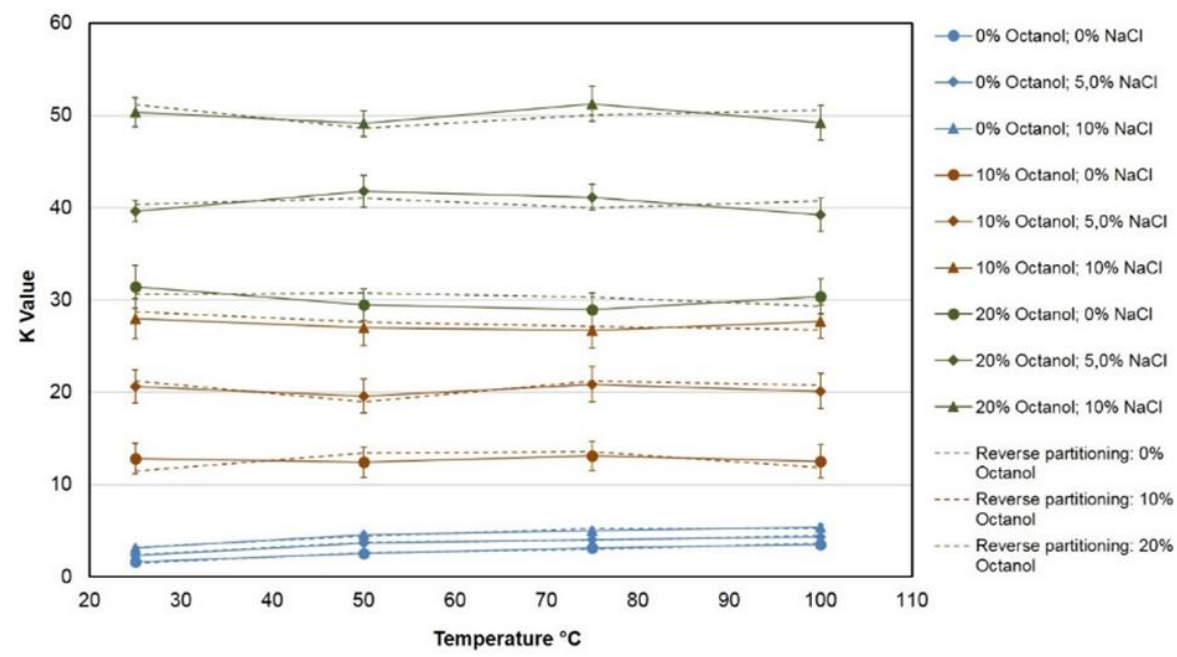

Figure 40. K-values of 4-chlorobenzyl alcohol $\pm \sigma(\mathrm{N}=3)$ as function of temperature, composition of the hydrocarbon phase and salinity. 


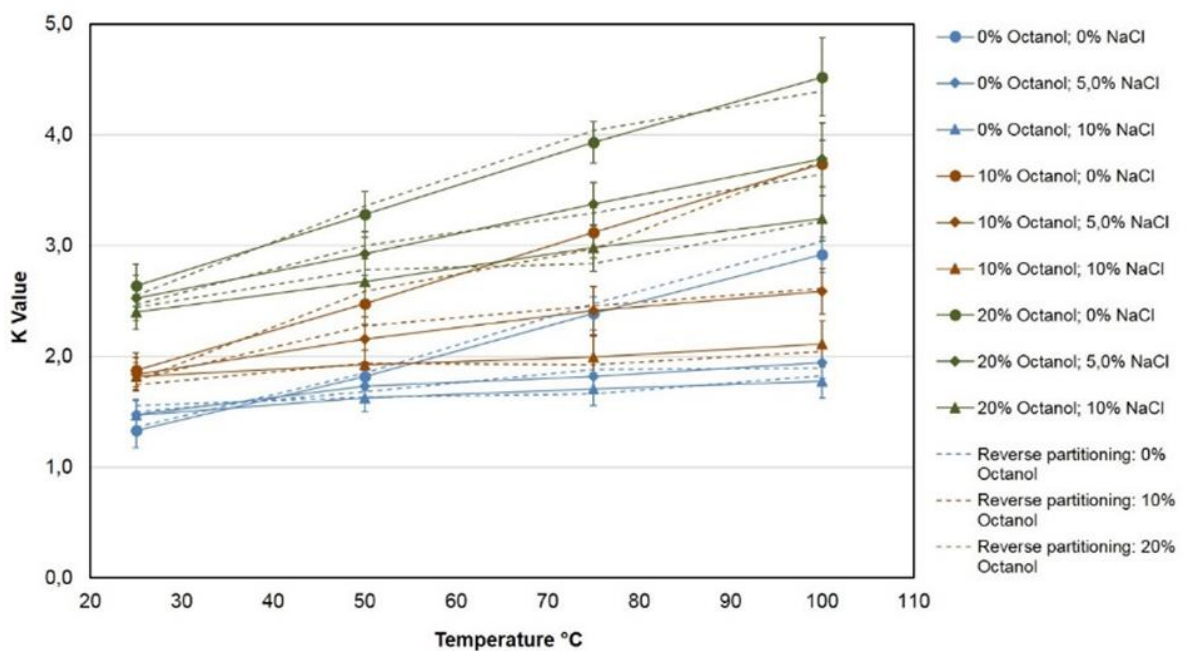

Figure 41. K-values of 2,6-dichlorobenzyl alcohol $\pm \sigma(\mathrm{N}=3)$ as function of temperature, composition of the hydrocarbon phase and salinity.

The presence of octanol in the hydrocarbon phase leads to higher Kvalues. In fact, this is the most relevant factor influencing the partitioning behaviour. Octanol is a linear molecule with a polar end and a linear hydrophobic chain. This molecule can establish lipophilic interactions (Van-der-Waals forces), as well as dipole and hydrogen bridges (due to its $\mathrm{OH}$ group), which lead to higher dissolution of the phase-partitioning compounds, since they all can establish hydrogen bridges and have core structures with some degree of lipophilicity (Paper VII).

Results show that the K-values of 4METBZOH and 4ClBZOH (Figures 38 and 40) are apparently only influenced by temperature in the experiments where the aqueous phase is deionised water. This behaviour is observed for the 2 candidates with substituents in the para-position. This reduces the polarity of the two molecules as they both have electronegative substituents (methoxy and chloro groups, respectively), which can justify the small temperature effect. More neutrality means more pre-disposition to leave the polar aqueous phase (Paper VII). 
Non-ionized organic compounds are dissolved in water due to dipole interactions and/or the establishment of hydrogen bonds. The reduction of solubility of organic compounds in water caused by increasing salinity of the solution is commonly known as the "salting-out effect" and is caused by the disturbance induce by the presence of ions on the dipole interactions and the hydrogen bonds between water molecules and the non-ionic solute molecules. Thus, increasing the concentration of ions has as a general consequence that the K-values are increased (Paper VII). This effect was also explored in the analytical method presented in Paper VI.

The K-value of $26 \mathrm{ClBZOH}$ decreases with increasing salinity. For solubility in water (single-phase), the same "salting-in" effect has been reported for cyclic organic compounds with chlorate and amino groups (Xie et al., 1997), benzene, naphthalene, and biphenyl (Janado et al., 1983), and 1,2-benzantracene and benzo[a]pyrene (Whitehouse, 1985). The "salting-in" observed for $26 \mathrm{ClBZOH}$ in the partitioning experiments can be explained by the possible formation of a coordinated sphere of ions around the molecule due to its dipolar nature or due to possible existence of lower-efficiency micro-emulsions in the phase interface caused by the higher density of the aqueous phase as consequence of the higher salt concentration (Paper VII). The latter has been proposed by (Makoś et al., 2018) to explain the reduction of yield in liquid-liquid extraction of aromatic and aliphatic organic acids from water.

The results show good agreement between the K-values obtained in the experiments where the PITT tracer candidates were originally dissolved in the aqueous phase and those where they were dissolved in the hydrocarbon phases (see dashed lines in Figures $35-41$ ). This shows that the partitioning is constant and reversible for fixed conditions of temperature, salinity, and hydrocarbon composition. In addition, the partitioning is independent of the initial concentrations of the potential tracer compounds and in which phase they are initially dissolved (Paper VII). 
$\mathrm{NaCl}$ is the primary constituent of brines in oilfields. However, common oilfield brine concentrations and compositions vary considerably. Other major components may be the following divalent ions: $\mathrm{Ca}^{2+}, \mathrm{Mg}^{2+}, \mathrm{Fe}^{2+}$, $\mathrm{Ba}^{2+}, \mathrm{CO}_{3}{ }^{2-}$ and $\mathrm{SO}_{4}{ }^{2-}$ (Fakhru'l-Razi et al., 2009). Additionally, the valency of ions is known to influence many other parameters in an oilfield such as wettability for instance. It is therefore of interest to examine whether the presence of divalent ions had any influence on the $\mathrm{K}$-values. Figure 42 presents the results obtained.

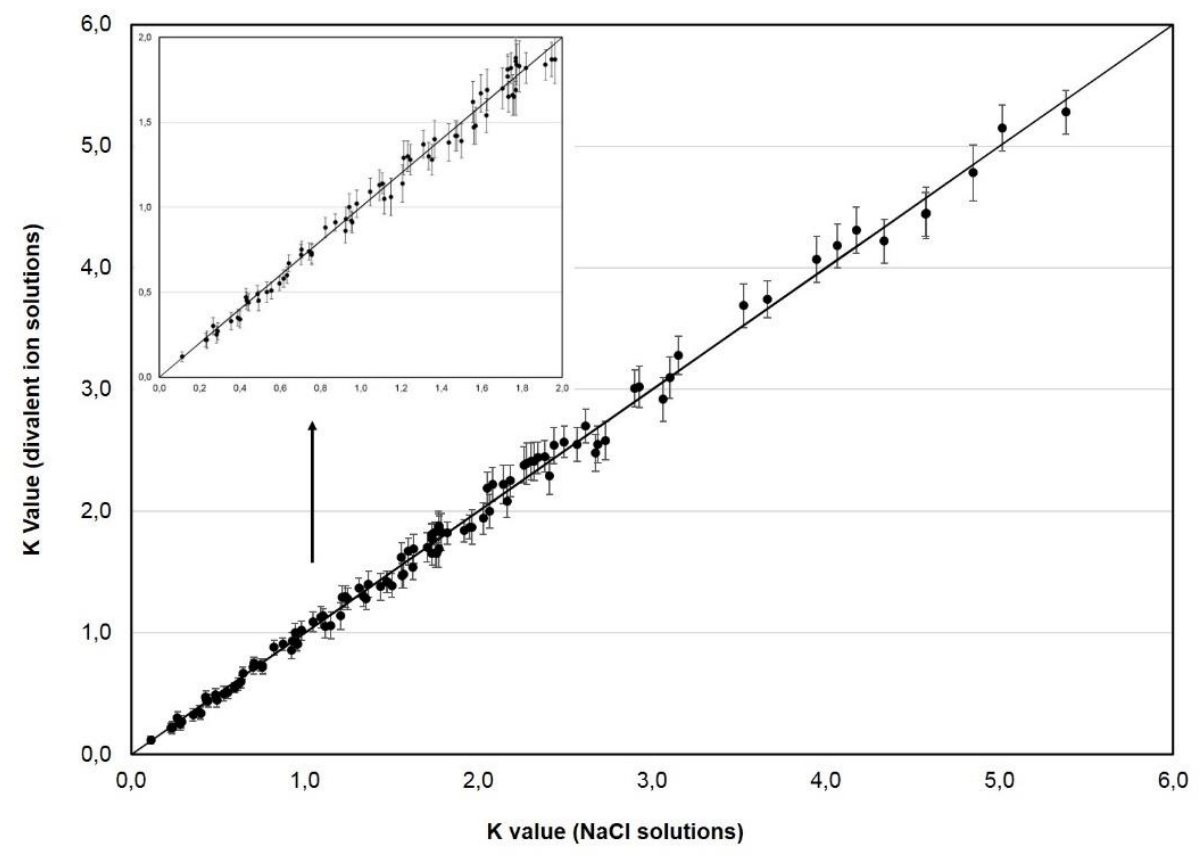

Figure 42. K-values $\pm \sigma(\mathrm{N}=3)$ of the tested compounds in experiments with an aqueous phase containing divalent ions vs the K-values in experiments with an aqueous phase containing only $\mathrm{NaCl}$ for the same ion strength.

The hydrocarbon phase for the results in Fig. 42 was composed of 80 vol\% iso-octane and 20 vol\% toluene. Each point obtained from the same compound at the same conditions of temperature and ionic strength (I) (calculated using Eq. 4) of the aqueous phase. Results suggest that the partitioning of the tracer candidates between phases is not dependent on 
the valency of the ions. No significant difference is encountered between the K-values obtained in the experiments where the ion strength of the water is made-up of divalent or exclusively monovalent ions. The ion strength itself of the aqueous phase appears to be the relevant parameter (Paper VII).

The same partition experiments as performed with synthetic hydrocarbon mixtures were carried out using black oils from the Norwegian oil fields Heidrun, Norne, Snorre, Ekofisk and Eldfisk. Results (not shown here but available in Paper VII) show lower variation of the K-values of the 7 investigated PITT tracer candidates within the studied conditions when compared with the ones from experiments with the synthetic hydrocarbon mixture. This again proves the influence of the parameters studied on the partitioning phenomena. Also, the Kvalues obtained show the applicability of the PITT tracer candidates on the NCS, considering the salinity of the water used there in the waterflooded oilfields. Furthermore, the same trends of K-values as function of the evaluated system parameters found in the experiments with a synthetic hydrocarbon mixture were observed in the experiments with real oil, confirming the complex nature of the partitioning phenomena and its dependency on the chemical nature of the substances (Paper VII).

\subsection{Dynamic flooding properties of the stable PITT tracer candidates}

Flooding experiments (described in section 2.3.6.) in water-saturated and known residual oil saturated sandstone and chalk cores were performed. HTO was used as the reference passive water tracer. These experiments had as the primary goals to evaluate deviations from ideal behaviour exhibited by the PITT tracer candidates while travelling through porous media, and to verify their accuracy in measuring the Sor value. 


\subsubsection{Flooding experiments in water saturated cores}

Results in section 3.1. show that the 7 PITT tracer candidates tested in the present section have no irreversible or long-term adsorption as well as no degradation in the presence of rock materials. However, other types of interactions, such as short-term reversible adsorption, could lead to different flow behaviour through a porous medium relatively to a passive water tracer. In a water saturated system, the production curves of the PITT tracer candidates should be equal to the one of HTO. If HTO flows through the cores exactly like the normal water does, then it samples the total pore volume swept by the injected water (we are disregarding any possible isotopic exchange of tritium with possible hydrogen molecules in the rock substrate). Figure 43 shows E(t) (Eq. 5) of HTO and the PITT tracer candidates as function of the total eluted mobile phase in the flooding experiment with a water saturated sandstone core.

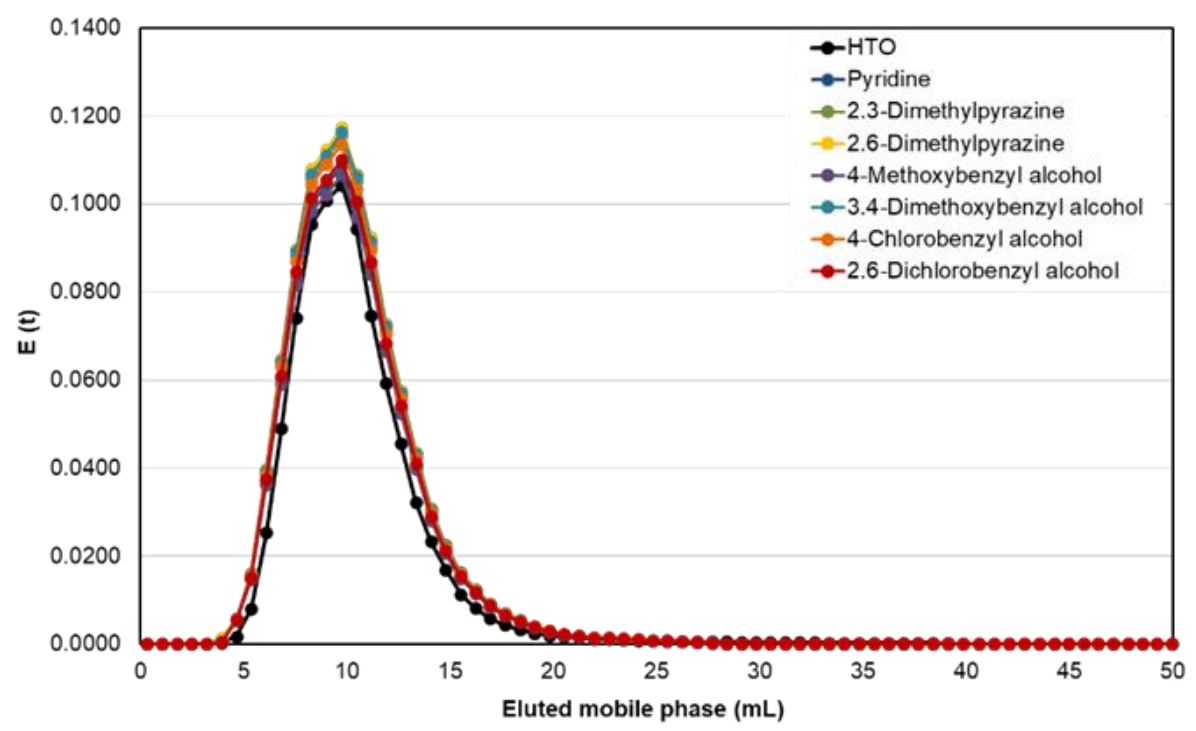

Figure 43. E (t) vs eluted mobile phase in the flooding experiments with a water-saturated sandstone core. 
The form of presentation of the production curve in Figure 43, allows to visually obtain an idea of the total recovery of each of the injected compounds (Eq. 7). HTO as lower recovery values than the PITT tracer candidates. Recoveries were calculated for all tracers injected and results are presented in Table 10.

The shape of the produced curves of all PITT tracer candidates appears to be the same as HTO. Additionally, the time of arrival (the first moment when the tracers are detected in the produced fluid) is also the same. This suggests that no significant difference in the flooding properties exists between the PITT tracer candidates and HTO. The PITT tracer candidates travel through the same swept pore volume as water does. There is no sign of reversible sorption to the sandstone rock. Table 10 also presents the total pore volume of the sandstone core calculated from the recovery of each of the tracer candidates.

Figure 44 shows E(t) (Eq. 5) of HTO and the PITT tracer candidates as function of the total eluted mobile phase in the flooding experiment with a water saturated chalk core.

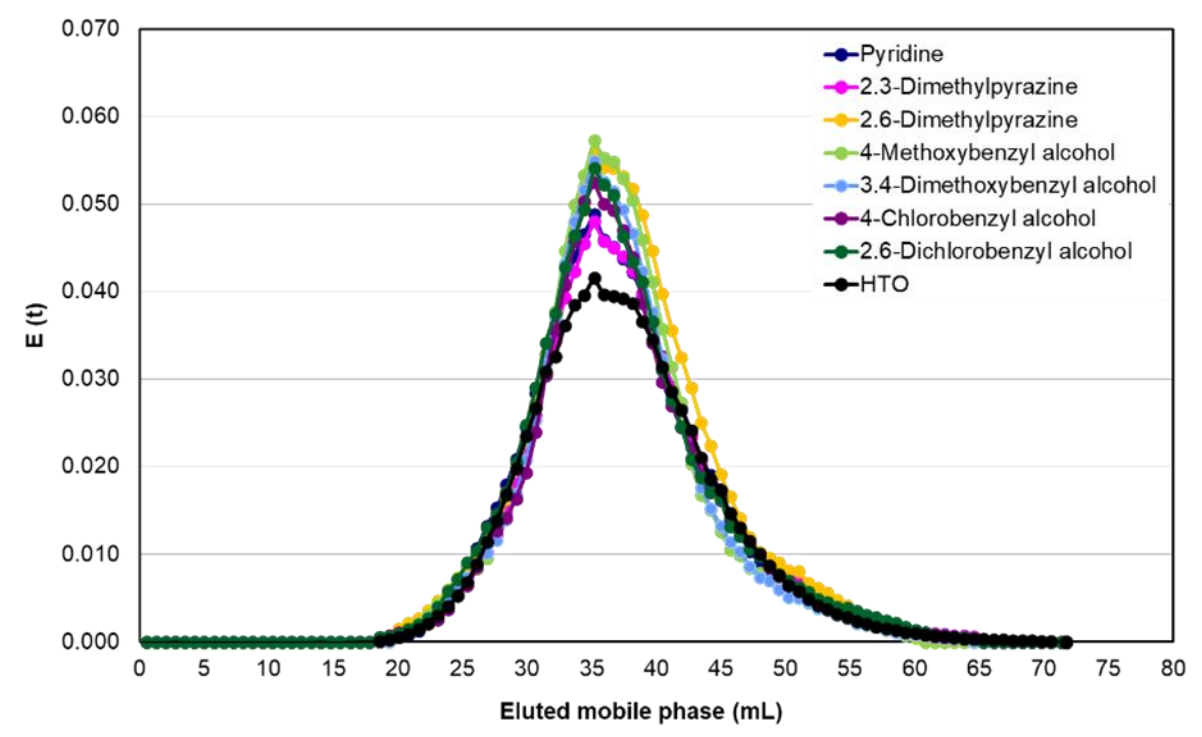

Figure 44. E (t) vs eluted mobile phase in the flooding experiments with a water-saturated chalk core. 
All the considerations presented about the experiments with the water saturated sandstone core are also applicable for the water saturated chalk core. Results suggest that the PITT tracer candidates travel through the same swept pore volume as the water does. The shapes of the curves are the same as well as first moment of the curves for the various tracers. Again, the recovery of HTO is lower than the recovery of the tracer candidates. Table 10 also contains all relevant calculated values for the flooding experiments in water saturated chalk.

Table 10 - Recoveries of HTO, the PITT tracer candidates and total pore volume calculated from each of their respective production curves.

\begin{tabular}{|c|c|c|c|c|}
\hline \multirow[b]{2}{*}{ Tracer } & \multicolumn{2}{|c|}{ Berea sandstone core } & \multicolumn{2}{|c|}{ Stevns Klint chalk core } \\
\hline & $\begin{array}{c}\text { Recovery } \\
(\%)\end{array}$ & $\begin{array}{l}\text { Total core pore } \\
\text { volume }(\mathrm{mL})\end{array}$ & $\begin{array}{c}\text { Recovery } \\
(\%)\end{array}$ & $\begin{array}{c}\text { Total core pore } \\
\text { volume }(\mathrm{mL})\end{array}$ \\
\hline HTO & 86,1 & $10,4 \pm 0,2$ & 86,9 & $37,6 \pm 0,4$ \\
\hline PYR & 97,8 & $10,3 \pm 0,2$ & 94,2 & $37,2 \pm 0,4$ \\
\hline 23MPRZ & 103 & $10,3 \pm 0,2$ & 91,8 & $37,2 \pm 0,4$ \\
\hline 26MPRZ & 105 & $10,5 \pm 0,2$ & 106 & $37,8 \pm 0,4$ \\
\hline 4METBZOH & 95,7 & $10,4 \pm 0,2$ & 97,7 & $37,4 \pm 0,4$ \\
\hline 34METBZOH & 104 & $10,5 \pm 0,2$ & 92,5 & $37,8 \pm 0,4$ \\
\hline 4ClBZOH & 102 & $10,3 \pm 0,2$ & 92,4 & $37,6 \pm 0,4$ \\
\hline 26ClBZOH & 98,8 & $10,4 \pm 0,2$ & 97,4 & $37,4 \pm 0,4$ \\
\hline
\end{tabular}

The pore volume obtained from each of the production curves is approximately the same both for the sandstone and chalk cores (HTO produces the "reference" value). No significant differences were encountered, further validating the conclusion that the PITT tracer candidates sweep the same volume as the water does. The PITT tracer candidates exhibit good recoveries. The variations in the recoveries are all easily explained with the uncertainty in the concentrations determined by the analytical method used together with the errors that arise from the discrete integration of the production data. The recoveries of HTO seems to be abnormally low. Thus, further investigation into this effect was 
performed. It was concluded that the ratio of the volume of scintillation solution to the volume of the sample should have been increased relatively to the one used one in the present analytical procedure $(50 \mu \mathrm{L}$ sample $+5 \mathrm{~mL}$ scintillation solution). The quenching effect caused by water, if not corrected for, causes a lower counting efficiency and thereby a seemingly lower recovery. HTO was re-measured in 5 random samples from the experiments on the sandstone core by adding $10 \mathrm{~mL}$ of scintillation solution to $50 \mu \mathrm{L}$ of sample. This yielded a higher counting rate. Correcting the previously obtained results with the improvement in measurement, HTO's recovery would become $97,4 \%$.

To better explain abnormalities in similar experiments (Bjørnstad et al., 1994) presented a method to visualise if the rates of production of the tracer candidates differ significantly from the ones of the reference tracer at different moments of the production. This allows to identify patterns that could be missed by the results presented so far. This method consists of normalising the concentration of the reference tracer and the tracer candidates to the value expected if the recovery were to be $100 \%$. The difference between the normalised to $100 \%$ recovery residence time distributions $[\mathrm{EN}(\mathrm{t})]$ of the tracer candidates and the reference tracer is then plotted vs the pore volume. Figures 45 and 46 present this analysis for each of the PITT tracer candidates. 

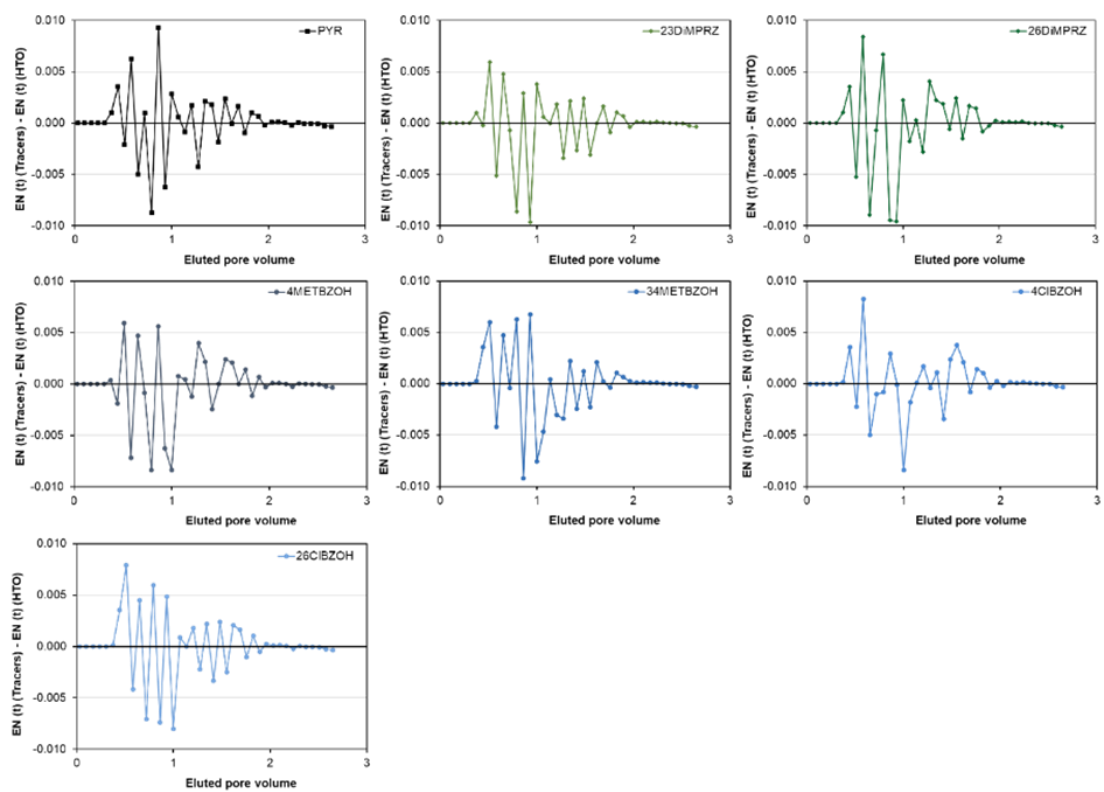

Figure 45. EN (t) of the PITT tracer candidates - EN (t) of HTO vs eluted pore volume in the water saturated sandstone core.
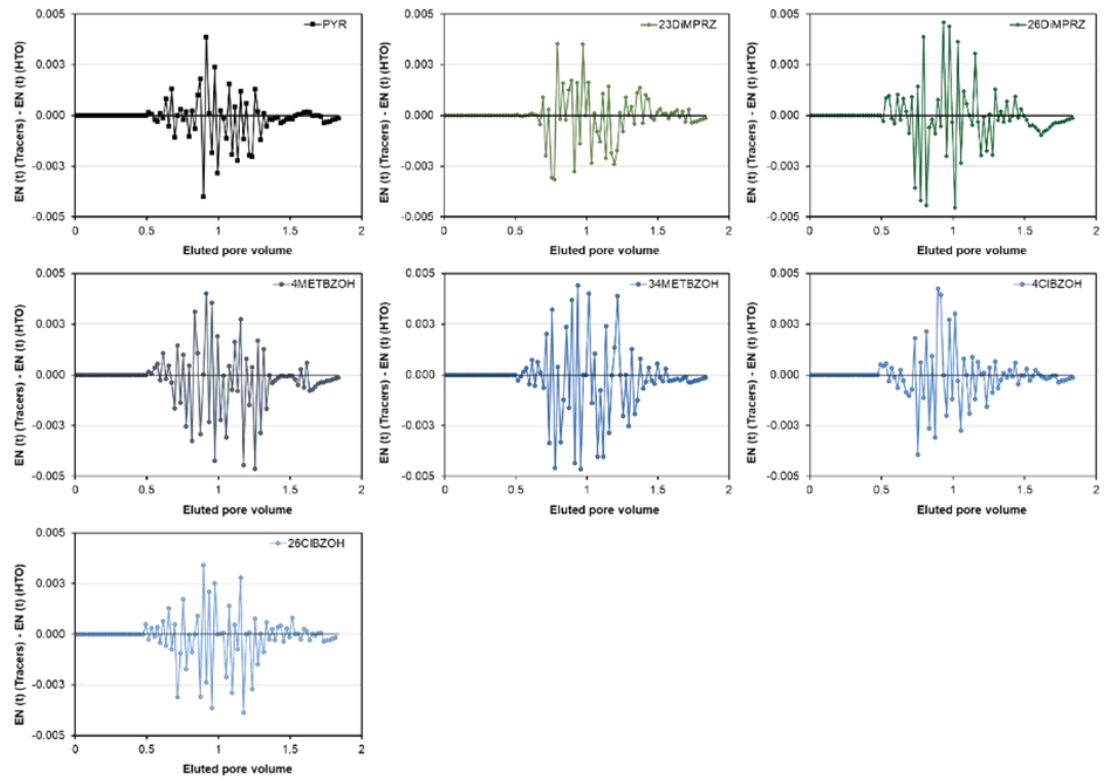

Figure 46. EN (t) of the PITT tracer candidates - EN (t) of HTO vs eluted pore volume in the water saturated chalk core. 
No visible pattern is observable in Figures 45 and 46, though the largest differences in EN ( $\mathrm{t}$ ) tend to occur before 1 pore volume is eluted. This is most likely because the variations in concentrations of the samples are more pronounced before 1 pore volume (the maximum height of the curves) than after this point. Even so, the differences are not systematically negative or positive, nor exhibit any obvious trend. This further validates that the flooding behaviour of the PITT tracer candidates in the water-saturated cores is water identical, i.e., the tested molecules follow the water percolation through the cores.

\subsubsection{Flooding experiments in cores in Sor conditions}

SoR in the prepared cores was determined by two methods: (1) through a mass balance of the injected oil (black oil from Heidrun field) and (2) by the reduction in pore volume swept by HTO relatively to the one obtained in the water saturated experiments. Figures 47 and 48 present $\mathrm{E}(\mathrm{t})$ of HTO and the PITT tracer candidates as function of the total eluted mobile phase in the flooding experiment with sandstone and chalk cores under $\mathrm{S}_{\mathrm{OR}}$ conditions, respectively. 


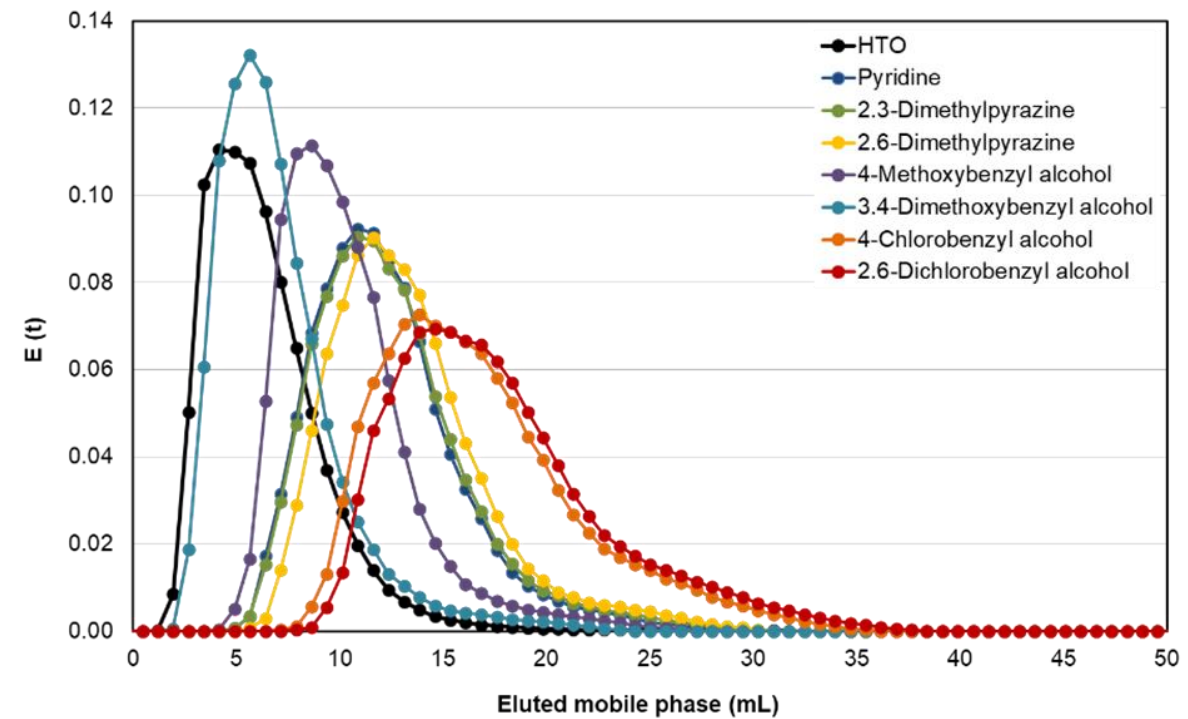

Figure 47. E ( $t$ ) vs eluted mobile phase in the flooding experiments with the sandstone core in SOR conditions.

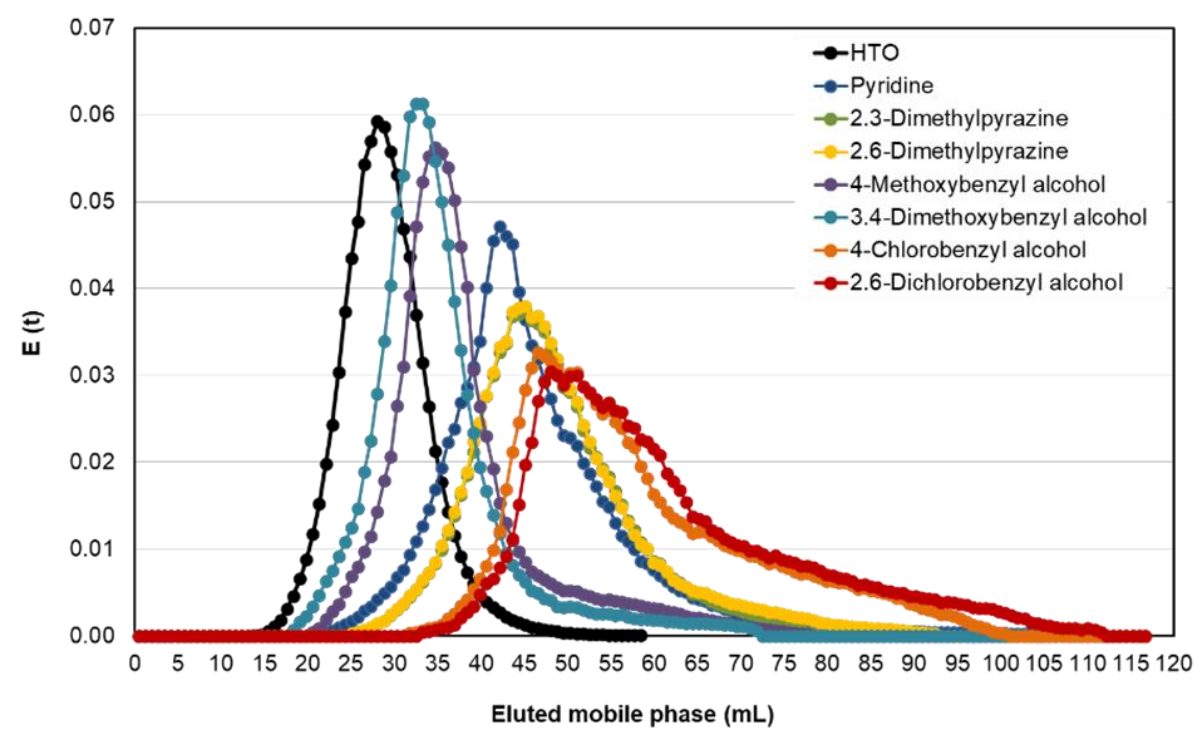

Figure 48. E ( $t$ ) vs eluted mobile phase in the flooding experiments with the chalk core in SoR conditions. 
Results show a delay in production of the PITT tracer candidates relatively to HTO. This proves that they are partitioning into the oil phase and redissolving in water. The longer the delay the lower and wider is the peak. This would be expected as increasing K-values produce longer retentions. The K-values presented in Paper VII for the PITT tracer candidates vs Heidrun oil were used to calculate the SoR determined by each candidate at $50 \%$ and $70 \%$ of their recovery. Studies by (Tang, 1992) proved that any two same moments of the production curves could be used to calculate $S_{O R}$ using Eq. 3. Tables 11 and 12 present the $S_{O R}$ measurements for the experiments in sandstone and chalk cores, respectively. $\mathrm{T}_{\mathrm{W}}$ refers to $\mathrm{HTO}$ and $\mathrm{T}_{\mathrm{R}}$ refers to the PITT tracer candidates.

Table 11 - Sor values determined by each of tracer candidates at $50 \%$ and $70 \%$ recovery in the sandstone core in SoR conditions.

\begin{tabular}{|c|c|c|c|c|c|}
\hline & & \multicolumn{2}{|c|}{$\mathrm{T}_{\mathrm{W}}=54,65 \mathrm{~min}$} & \multicolumn{2}{|c|}{$\mathrm{T}_{\mathrm{W}}=74,90 \mathrm{~min}$} \\
\hline Tracer & $\begin{array}{l}\text { K vs. (Heidrun } \\
\text { oil) }\end{array}$ & $\begin{array}{c}\mathrm{T}_{\mathrm{R}}(50 \% \\
\text { recovery) } \\
\quad(\min )\end{array}$ & $\mathrm{S}_{\mathrm{OR}}(\%)$ & $\begin{array}{c}\mathrm{T}_{\mathrm{R}}(70 \% \\
\text { recovery) } \\
\quad(\min )\end{array}$ & SOR $(\%)$ \\
\hline PYR & 2,01 & 127,1 & $39,7 \pm 0,4$ & 175,2 & $40,0 \pm 0,4$ \\
\hline 23MPRZ & 2,11 & 129,8 & $39,5 \pm 0,4$ & 179,6 & $39,8 \pm 0,4$ \\
\hline 26MPRZ & 2,39 & 141,3 & $39,9 \pm 0,4$ & 192,7 & $39,7 \pm 0,4$ \\
\hline 4METBZOH & 1,14 & 96,26 & $40,0 \pm 0,4$ & 132,1 & $40,1 \pm 0,4$ \\
\hline 34METBZOH & 0,92 & 88,50 & $40,2 \pm 0,4$ & 120,6 & $39,9 \pm 0,4$ \\
\hline $4 \mathrm{ClBZOH}$ & 3,12 & 167,1 & $39,7 \pm 0,4$ & 227,9 & $39,6 \pm 0,4$ \\
\hline $26 \mathrm{ClBZOH}$ & 3,31 & 171,7 & $39,3 \pm 0,4$ & 235,8 & $39,4 \pm 0,4$ \\
\hline \multicolumn{6}{|c|}{ Sor from the balance to the injected oil $=\mathbf{3 9 , 5} \%$} \\
\hline Sor from the $r$ & in water nore & easur & $\mathrm{TO}=\mathbf{3 9}$ & & \\
\hline
\end{tabular}


Table 12 - Sor values determined by each of tracer candidates at $50 \%$ and $70 \%$ recovery in the chalk core in Sor conditions.

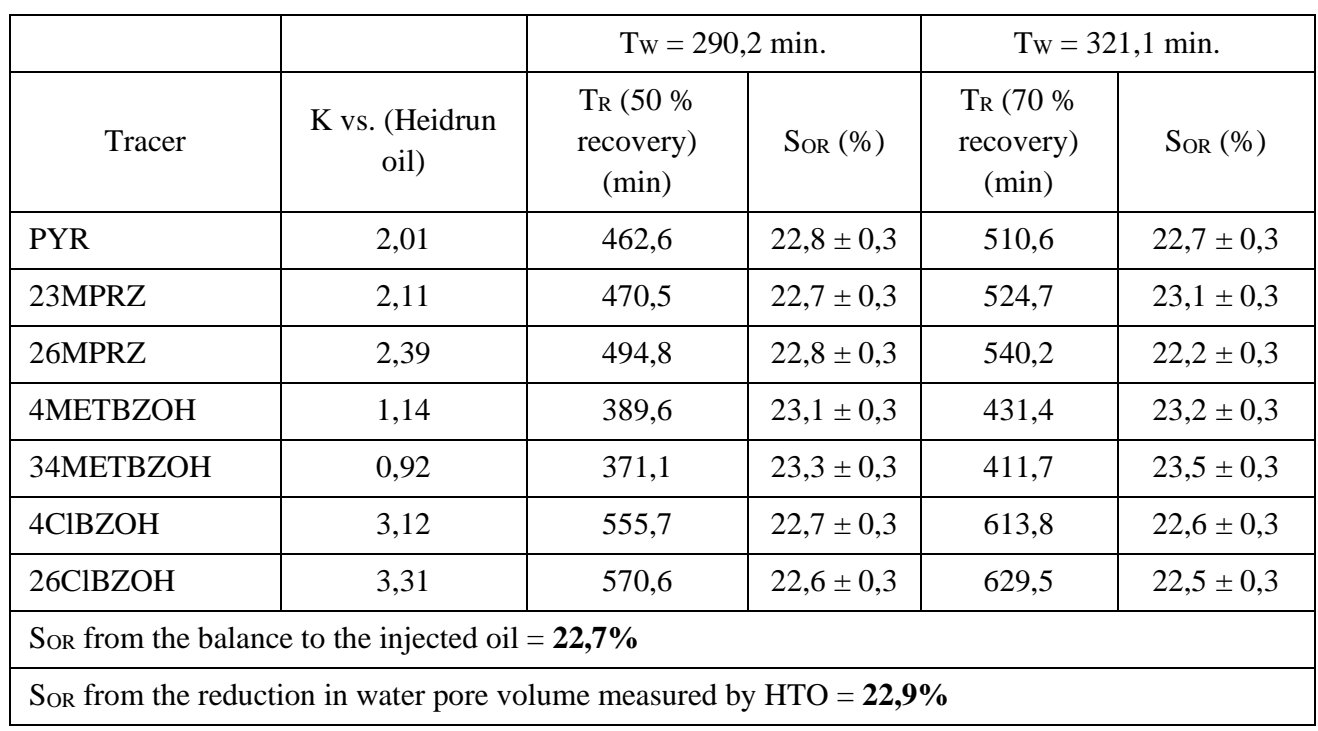

Good agreement was obtained between the measurements of $S_{\text {OR }}$ by each of the PITT tracer candidates and the SoR-values obtained during preparation of the cores. In both experiments, the PITT tracer candidates with lower K-values (4METBZOH and 34METBZOH) measure a value of Sor that seems systematically higher than any other obtained. This is likely due to the lowest separation relatively to the water tracer. However, both compounds yield acceptable results. 4CIBZOH and $26 \mathrm{ClBZOH}$ systematically yield lower values, though acceptable, in comparison with all other compounds. This is likely due to the tailing effect caused by the higher K-values. Globally, it is safe to argue that the PITT tracer candidates are able to produce accurate results. 


\section{Conclusion and future work}

\subsection{Conclusion}

The future of oil production on the NCS will increasingly rely on IOR projects. These projects are often costly and lead to alterations in the reservoir properties that again lead to the need for deep updating of the reservoir models. The most thorough possible knowledge and understanding of the reservoir should serve as basis for the planning of IOR operations. The PITT provides valuable information for identification of IOR targets and evaluation of IOR projects. Since its introduction to the oil industry in 1971, the PITT was received with scepticism and used at a very limited scale. One of the main reasons for this is the lack of tracers properly developed for this application. Thus, the overall objective of the present study was to introduce a comprehensive methodology for selection and testing of new oil/water partitioning tracers for inter-well applications and apply this procedure to a group of molecules with promising characteristics. The author hopes that the results obtained in this thesis will help to disseminate the use of PITT by the oil industry. A thorough investigation was performed based on static stability experiments, characterisation of the variations of the $\mathrm{K}$-values, dynamic flooding experiments, and the development of analytical methods to analyse the PITT tracer candidates in real produced waters from oilfields. Sequentially, these experiments produced a group of 7 compounds that passed all the qualification steps and are analysable in the low ng/L range. Each step of the methodology proposed resulted in valuable conclusions, that combined ensure very low probability of failure from the use of 2,3-dimethylpyrazine; 2,6-dimethylpyrazine; pyridine; 4-chlorobenzyl alcohol; 2,6-dichlorobenzyl alcohol; 4methoxybenzyl alcohol and 3,4-dimethoxybenzyl alcohol as oil/water partitioning tracers in PITTs.

The static stability experiments proved effective in evaluating the thermal stability of the PITT tracer candidates as well as in uncovering 
significant interactions with reservoir rock materials. First and foremost, it was demonstrated that the stability of a given molecule is intimately related to its substituents. 16 Compounds from 4 "families" of chemicals were evaluated and only five of these were found to be fully stable up to $150{ }^{\circ} \mathrm{C}$ for 12 weeks. These five compounds enclose molecules from 3 of the 4 families of chemicals tested. The most paradigmatic example is the case of the benzyl alcohols: the chlorinated benzyl alcohols are fully stable, however the methoxy substituted benzyl alcohols exhibit a sudden and fast degradation above $125{ }^{\circ} \mathrm{C}$. The methoxy benzyl alcohols were investigated further, as their upper limit of thermal stability allows for their use in most oilfields. Furthermore, 4-hydroxybenzyl alcohol degrades even at low temperatures at the same experimental conditions, further showing the importance of the substituents in the overall stability of a molecule. It was verified that the thermally driven degradation can be explored to retrieve additional data from the inter-well region. The degradation of 2 molecules fits a pseudo-first order degradation kinetic, thus allowing for their use to retrieve information about temperature in the swept volumes between injector/producer well-pairs. This information can be important for reservoir simulation where chemical EOR processes are modelled. Results show that 2-methoxypyrazine and 4-methoxypyridine are strongly affected by the presence of kaolinite, however the nature of the interaction is not clear, with adsorption or catalytic activity form the clay being the most likely explanations. The same effect was also observed in combination with $\mathrm{pH}$ for 3hydroxypyridine. This data opens the possibility of using tracers to retrieve geochemical information from the inter-well region and should be explored further.

An analytical method for analysis of the thermally stable PITT tracer candidates in real produced waters was developed. This method proved effective in the identification and quantification of all 7 compounds in concentrations in the low $\mathrm{ng} / \mathrm{L}$ range, while confirming the upfront absence of all molecules in 8 samples of produced waters from various oil fields at NCS. Furthermore, SPME was, to the best of the author's 
knowledge, introduced for the first time to the analysis of produced waters from oilfields. This sample preparation technique allows to significantly reduce the time and amount of chemicals used by the commonly used sample preparation techniques, as well as the amount of sample volume required for analysis. The method presented consists of DI-HS-SPME-GC-MS/MS as requires just $5 \mathrm{~mL}$ of sample. The PITT tracer candidates identified in the present study can be used on field in small amounts and their analysis also requires small volumes of sample, a factor that can be particularly important in offshore operations because of logistic issues. Building accurately enough production curves from an inter-well tracer test typically requires several hundred samples to be analysed.

The K-value of an oil/water partitioning tracer is influenced by several parameters of the system (oil reservoir). The methodology of study and results presented in this thesis show that the tested compounds exhibit in fact constant and reversible phase partitioning. This is of the utmost importance, as it is one of the basic assumptions behind the concept of the PITT itself. It was verified that the chemical nature and position of the substituents of a molecule influences the extent of its partitioning, and may even, as in the case of chlorinated benzyl alcohols, invert trends as function of system parameters (salinity was found to cause "saltingin" of 2,6-dichlorobenzyl alcohol). The value of $\mathrm{K}$ is primarily influenced by the ionic strength of the aqueous phase and the composition of the organic phase. Temperature will make the K-value vary in a larger or smaller extent depending on the specific characteristics of the molecule in question. Results suggest that the valency of the ions present in the aqueous phase is irrelevant for the $\mathrm{K}$ value, that seems to be influenced just by the ionic strength. In general, the increase of the ionic strength of the aqueous phase, higher contents of polar and aliphatic compounds in the hydrocarbon phase and increase of temperature lead to higher K-values. A notable exception when it comes to ionic strength is 2,6-dichlorobenzyl alcohol, due to dipolar nature and the molecule and, consequently, strong dipole interactions 
with the ions in the aqueous phase. $\mathrm{pH}$ has no influence on the phase partitioning, except when molecules have an acid or basic character under the relevant $\mathrm{pH}$ conditions. This was observed for pyridine. All the data included in this chapter of the present study (Paper VII) can be incorporated in simulation models that can be used to determine the results of PITT with higher accuracy.

The dynamic flooding experiments have proven that, on a lab scale, the PITT tracer candidates tested exhibit the required characteristics to be used on the field. In absence of hydrocarbons (water saturated system) they flow through the porous media like water does. Similar production profiles were obtained for the investigated molecules as for the reference water tracer (tritiated water) and good agreement was found between the pore volume measured by the PITT tracer candidates and HTO, both on sandstone and chalk cores. In the experiments with the cores prepared to Sor conditions, and using the values of K determined in Paper VII, good agreement was found between the oil saturation measured by each of the tracer candidates and the values obtained during core preparation. The core concept of the PITT implies the existence of local equilibrium distribution of the oil/water partitioning tracer along the flooded flowpath (constant and reversible partition). Results suggest this verifies with the PITT tracer candidates investigated by the present work. Although, these results come experiments performed on cylindrical cores with dimensions of $38,1 \mathrm{~mm} \times 70 \mathrm{~mm}$, they suggest that the group of 7 compounds investigated is ready for deployment on the field.

Overall, the methodology developed and presented in this thesis and the application of this methodology to a group of molecules with promising characteristics as new oil/water partitioning tracer candidates for interwell applications, produced a group of seven compounds ready to be tested on the field. A thorough understanding was gained on how the respective K-values vary, and an analytical method for their analysis in production waters was developed and is ready to be used. Additionally, many of the results and findings of the present study are directly applicable to the SWCTT. Thus, it is the author's hope to see the 
compounds presented here used on the Norwegian continental shelf soon.

\subsection{Future work}

The concept of the PITT was introduced in 1971. Plenty of work to improve the deployment and interpretation of this test has been done, and a robust methodology for development of new PITT tracers is presented in this thesis. However, very little work has been done focusing on the fundamentals of the PITT. One of the core assumptions of this test is that the equilibrium distribution of the phase-partitioning tracer between the hydrocarbon and aqueous phase happens instantaneously when both phases come in contact and that this translates into local equilibria conditions along the whole swept volume. Even if this is verified from laboratory experiments in cores of consolidated sedimentary rock, it is unclear whether it can be extrapolated for the large volumes sampled by a PITT. Diffusion effects influenced by the flowing aqueous phase can disturb the establishment of the equilibrium distribution of the tracers. Another aspect is that the concept of PITT does not account for the ratio volume of oil contactable by water/area of oil contactable by water. Further studies should be performed on the fundamental premise of the PITT to evaluate how and if diffusion of the tracers in the hydrocarbon phase and the ratio volume/area has effect on the accuracy of the measured Sor.

Digitalisation is an ever-growing trend in the oil industry that translates into increases the financial turnover. Including tracer data on reservoir models significantly improves the understanding of the reservoir. Thus, fully automated online/inline tracer analysis methods that produce tracer curves in real-time would be a huge breakthrough for tracer technology. Available technologies developed for bio-medical and environmental applications, such as biosensors, offer good insights on the path forward to achieve this goal. Thus, more effort should be put into the development of such systems. 
Examples in the present study as well as in other works, for example (Strand et al., 2006), show that active tracers sensitive to geochemical features of the rock formation can be used to retrieve pertinent reservoir information. The industry has for long wanted to measure wettability in the inter-well region, and it is the author's conviction that this goal can be achieved with further investigation. Efforts should be put in place to fully understand the interactions of selected groups of molecules and reservoir rock materials so that the observed effects can be used for the development of tracers that produce a signal which can be directly related to the area of water- or oil-wet rock in the volume they swept. Tracer technology has proven to be one of the most reliable technologies to obtain data about the inter-well region of oilfields. Thus, expanding the information that tracer technology can deliver is in the best interest of an industry that will rely increasingly on expensive production methods. 


\section{$5 \quad$ References}

Adams, T.B., Doull, J., Feron, V.J., Goodman, J.I., Marnett, L.J., Munro, I.C., Newberne, P.M., Portoghese, P.S., Smith, R.L., Waddell, W.J., Wagner, B.M., 2002. The FEMA GRAS assessment of pyrazine derivatives used as flavor ingredients. Food and Chemical Toxicology 40, 429-451.

Ahmad, M., Tasneem, M.A., Rafiq, M., Khan, I.H., Farooq, M., Sajjad, M.I., 2003. Interwell tracing by environmental isotopes at Fimkassar Oilfield, Pakistan. Applied radiation and isotopes : including data, instrumentation and methods for use in agriculture, industry and medicine 58, 611-619.

Ahsan, H.M., Breedlove, B.K., Piangrawee, S., Mian, M.R., Fetoh, A., Cosquer, G., Yamashita, M., 2018. Enhancement of electrocatalytic abilities for reducing carbon dioxide: functionalization with a redoxactive ligand-coordinated metal complex. Dalton Transactions 47, 11313-11316.

AlAbbad, M.A., Sanni, M.L., Kokal, S., Krivokapic, A., Dye, C., Dugstad, Ø., Hartvig, S.K., Huseby, O.K., 2018. A Step Change for Single-Well Chemical-Tracer Tests: Field Pilot Testing of New Sets of Novel Tracers. SPE-181408-PA Preprint, 13.

Alasalvar, C., Shahidi, F., Cadwallader, K.R., 2003. Comparison of natural and roasted Turkish tombul hazelnut (Corylus avellana L.) volatiles and flavor by DHA/GC/MS and descriptive sensory analysis. J. Agric. Food Chem. 51, 5067-5072.

Asadi, M., Shook, G.M., 2010. Application of Chemical Tracers in IOR: A Case History, North Africa Technical Conference and Exhibition. Society of Petroleum Engineers, Cairo, Egypt, p. 11. 
Austine, J., van Batenburg, D.W., Southwick, J.G., Zarubinska, M.A., Paramanathan, S., Bouwmeester, R.C.M., Kechut, N.I., Viig, S.O., Haugen, O.B., Brandvoll, Ø., 2015. Laboratory Evaluation of Inter-Well Partitioning Tracers for the Determination of Remaining Oil Saturation after ASP Flooding, SPE Asia Pacific Enhanced Oil Recovery Conference. Society of Petroleum Engineers, Kuala Lumpur, Malaysia, p. 14.

Babu Valapa, R., Loganathan, S., Pugazhenthi, G., Thomas, S., Varghese, T.O., 2017. Chapter 2 - An Overview of Polymer-Clay Nanocomposites, in: Jlassi, K., Chehimi, M.M., Thomas, S. (Eds.), ClayPolymer Nanocomposites. Elsevier, pp. 29-81.

Bae, E., Na, J.-G., Chung, S.H., Kim, H.S., Kim, S., 2010. Identification of about 30000 Chemical Components in Shale Oils by Electrospray Ionization (ESI) and Atmospheric Pressure Photoionization (APPI) Coupled with 15 T Fourier Transform Ion Cyclotron Resonance Mass Spectrometry (FT-ICR MS) and a Comparison to Conventional Oil. Energy \& Fuels 24, 2563-2569.

Bakaikina, N.V., Kenessov, B., Ul'yanovskii, N.V., Kosyakov, D.S., 2018. Quantification of transformation products of rocket fuel unsymmetrical dimethylhydrazine in soils using SPME and GC-MS. Talanta 184, 332-337.

Belver, C., Bañares Muñoz, M.A., Vicente, M.A., 2002. Chemical Activation of a Kaolinite under Acid and Alkaline Conditions. Chemistry of Materials 14, 2033-2043.

Bhattacharyya, K.G., Gupta, S.S., 2008. Adsorption of a few heavy metals on natural and modified kaolinite and montmorillonite: A review. Advances in Colloid and Interface Science 140, 114-131.

Bjørnstad, T., 1991. Selection of tracers for oil and gas evaluation, Norway, p. 48. 
Bjørnstad, T., Haugen, O.B., Hundere, I.A., 1994. Dynamic behavior of radio-labelled water tracer candidates for chalk reservoirs. Journal of Petroleum Science and Engineering 10, 223-238.

Blank, I., Sen, A., Grosch, W., 1992. Potent odorants of the roasted powder and brew of Arabica coffee Z. Lebensm.-Unters.-Forsch. 195, 239-245.

Boyaci, E., Gorynski, K., Viteri, C.R., Pawliszyn, J., 2016. A study of thin film solid phase microextraction methods for analysis of fluorinated benzoic acids in seawater. Journal of Chromatography A 1436, 51-58.

Buttery, R.G., Stern, D.J., Ling, L.C., 1994. Studies on flavor volatiles of some sweet corn products. J. Agric. Food Chem. 42, 791-795.

Câmara, J.S., Alves, M.A., Marques, J.C., 2006. Multivariate analysis for the classification and differentiation of Madeira wines according to main grape varieties. Talanta 68, 1512-1521.

Causin, E., Rochon, J., Marzorati, D., 1990. Field Measurements of Remaining Oil Saturation, SPE/DOE Enhanced Oil Recovery Symposium. Society of Petroleum Engineers, Tulsa, Oklahoma, p. 9.

Cerny, C., Grosch, W., 1994. Precursors of ethyldimethylpyrazine isomers and 2,3-diethyl-5-methylpyrazine formed in roasted beef $\mathrm{Z}$. Lebensm.-Unters.-Forsch. 198, 210-214.

Cooke, C.E.J., 1971. "Method of determining fluid saturations in reservoirs" (US Patent 3,590,923). Esso Production Research Company, USA.

de Hoffmann, E., Stroobant, V., 2007. Mass Spectrometry: Principles and Applications, 3rd Edition. John Wiley \& Sons, New York.

Deans, H.A., 1971. "Method of determining fluid saturations in reservoirs" (US Patent 3,623,842). Esso Production Research Company, USA. 
Deans, H.A., 1978. Using Chemical Tracers To Measure Fractional Flow And Saturation In-Situ, SPE Symposium on Improved Methods of Oil Recovery. Society of Petroleum Engineers, Tulsa, Oklahoma, p. 10.

Dearden, J.C., Bresnen, G.M., 1988. The Measurement of Partition Coefficients. Quantitative Structure-Activity Relationships 7, 133-144.

Dugstad, O., Viig, S., Krognes, B., Kleven, R., Huseby, O., 2013. Tracer monitoring of enhanced oil recovery projects, in: Haugan, A. (Ed.), Tracer 6 - the 6th International Conference on Tracers and Tracing Methods. E D P Sciences, Cedex A.

Esteruelas, M.A., García-Obregón, T., Herrero, J., Oliván, M., 2011. Osmium-Catalyzed Oxidation of Primary Alcohols with Molecular Oxygen. Organometallics 30, 6402-6407.

Eurachem, 2014. The Fitness for Purpose of Analytical Methods: A Laboratory Guide to Method Validation and Related Topics: Second edition. www.eurachem.org.

Fakhru'l-Razi, A., Pendashteh, A., Abdullah, L.C., Biak, D.R.A., Madaeni, S.S., Abidin, Z.Z., 2009. Review of technologies for oil and gas produced water treatment. Journal of Hazardous Materials 170, 530551.

Frank, D.C., Owen, C.M., Patterson, J., 2004. Solid phase microextraction (SPME) combined with gas-chromatography and olfactometry-mass spectrometry for characterization of cheese aroma compounds. LWT - Food Science and Technology 37, 139-154.

Gutiérrez, M.C., Carriazo, D., Ania, C.O., Parra, J.B., Ferrer, M.L., del Monte, F., 2011. Deep eutectic solvents as both precursors and structure directing agents in the synthesis of nitrogen doped hierarchical carbons highly suitable for $\mathrm{CO} 2$ capture. Energy \& Environmental Science 4, 3535-3544. 
Hartvig, S.K., Huseby, O., Yasin, V., Ogezi, O., Ernst, B., Reimann, S., Leonhardt, B., 2015. Use of a New Class of Partitioning Tracers to Assess EOR and IOR Potential in the Bockstedt Field.

Hedegaard, K., Graue, A., 2011. Does Wettability Affect the Strength of Chalk?, 45th U.S. Rock Mechanics / Geomechanics Symposium. American Rock Mechanics Association, San Francisco, California, p. 6.

Higashimoto, S., Kitao, N., Yoshida, N., Sakura, T., Azuma, M., Ohue, H., Sakata, Y., 2009. Selective photocatalytic oxidation of benzyl alcohol and its derivatives into corresponding aldehydes by molecular oxygen on titanium dioxide under visible light irradiation. Journal of Catalysis 266, 279-285.

Hirsh, A.J., Zhang, J., Zamurs, A., Fleegle, J., Thelin, W.R., Caldwell, R.A., Sabater, J.R., Abraham, W.M., Donowitz, M., Cha, B., Johnson, K.B., St. George, J.A., Johnson, M.R., Boucher, R.C., 2008. Pharmacological Properties of $<\mathrm{em}>\mathrm{N}</ \mathrm{em}>-(3,5-D i a m i n o-6-$ chloropyrazine-2-carbonyl)- $<\mathrm{em}>\mathrm{N}</ \mathrm{em}>^{\prime}-4-[4-(2,3-$

dihydroxypropoxy)phenyl]butyl-guanidine Methanesulfonate (552-02), a Novel Epithelial Sodium Channel Blocker with Potential Clinical Efficacy for Cystic Fibrosis Lung Disease. Journal of Pharmacology and Experimental Therapeutics 325, 77-88.

Hjuler, M.L., Fabricius, I.L., 2009. Engineering properties of chalk related to diagenetic variations of Upper Cretaceous onshore and offshore chalk in the North Sea area. Journal of Petroleum Science and Engineering 68, 151-170.

Janado, M., Yano, Y., Doi, Y., Sakamoto, H., 1983. Peculiar effects of alkali thiocyanates on the activity coefficients of aromatic hydrocarbons in water. Journal of Solution Chemistry 12, 741-754.

Jiang, S., 2012. Clay Minerals from the Perspective of Oil and Gas Exploration, in: Valaškova, M., Martynkova, G.S. (Eds.), Clay Minerals 
in Nature - Their Characterization, Modification and Application. InTech, Rijeka, p. Ch. 2.

Jousse, F., Jongen, T., Agterof, W., Russell, S., Braat, P., 2002. Simplified Kinetic Scheme of Flavor Formation by the Maillard Reaction. Journal of Food Science 67, 2534-2542.

Kaviani, S., Izadyar, M., 2018. The possibility of iron chelation therapy in the presence of different HPOs; a molecular approach to the noncovalent interactions and binding energies. Journal of Molecular Structure 1166, 448-455.

King, P.J., Morton, F., Sagarra, A., 1973. Chemistry and physics of petroleum, in: Hobson, G.D., Pohl, W. (Eds.), Modern petroleum technology, 4th ed. Applied Science Publishers, Barking, U.K.

Leo, A., Hansch, C., Elkins, D., 1971. Partition coefficients and their uses. Chemical Reviews 71, 525-616.

Li, C., Huang, Y., Dong, X., Sun, Z., Duan, X., Ren, B., Zheng, S., Dionysiou, D.D., 2019. Highly efficient activation of peroxymonosulfate by natural negatively-charged kaolinite with abundant hydroxyl groups for the degradation of atrazine. Applied Catalysis B: Environmental 247, $10-23$.

Lichtenberger, G.J., 1991. Field Applications of Interwell Tracers for Reservoir Characterization of Enhanced Oil Recovery Pilot Areas, SPE Production Operations Symposium. Society of Petroleum Engineers, Oklahoma City, Oklahoma.

Liu, S.-m., Wu, C.-H., Huang, H.-J., 1998. Toxicity and anaerobic biodegradability of pyridine and its derivatives under sulfidogenic conditions. Chemosphere 36, 2345-2357. 
Lucas, L.L., Unterweger, M.P., 2000. Comprehensive Review and Critical Evaluation of the Half-Life of Tritium. Journal of Research of the National Institute of Standards and Technology 105, 541-549.

Lucia, F.J., 1995. ROCK-FABRIC PETROPHYSICAL CLASSIFICATION OF CARBONATE PORE-SPACE FOR RESERVOIR CHARACTERIZATION. AAPG Bull.-Am. Assoc. Petr. Geol. 79, 1275-1300.

Luo, L., Kim, S.-W., Lee, H.-K., Kim, I.-D., Lee, H., Lee, J.-K., 2017. Anti-oxidative effects of 4-hydroxybenzyl alcohol in astrocytes confer protective effects in autocrine and paracrine manners. PLOS ONE 12, e0177322.

Madland, M.V., Hiorth, A., Omdal, E., Megawati, M., HildebrandHabel, T., Korsnes, R.I., Evje, S., Cathles, L.M., 2011. Chemical Alterations Induced by Rock-Fluid Interactions When Injecting Brines in High Porosity Chalks. Transport in Porous Media 87, 679-702.

Makoś, P., Fernandes, A., Przyjazny, A., Boczkaj, G., 2018. Sample preparation procedure using extraction and derivatization of carboxylic acids from aqueous samples by means of deep eutectic solvents for gas chromatographic-mass spectrometric analysis. Journal of Chromatography A 1555, 10-19.

Mannam, M.R., Devineni, S.R., Pavuluri, C.M., Chamarthi, N.R., Kottapalli, R.S.P., 2019. Urea and thiourea derivatives of 3(trifluoromethyl)-5,6,7,8-tetrahydro-[1, 2, 4]triazolo[4,3-a]pyrazine: Synthesis, characterization, antimicrobial activity and docking studies. Phosphorus, Sulfur, and Silicon and the Related Elements 194, 922-932.

Mantri, K., Komura, K., Kubota, Y., Sugi, Y., 2005. Friedel-Crafts alkylation of aromatics with benzyl alcohols catalyzed by rare earth metal triflates supported on MCM-41 mesoporous silica. Journal of Molecular Catalysis A: Chemical 236, 168-175. 
Mautner, F.A., Traber, M., Fischer, R.C., Reichmann, K., Vicente, R., 2018. Synthesis and characterization of pseudohalide-metal(II) complexes with 4-methoxypyridine as co-ligand. Polyhedron 144, 3035 .

Meinardus, H.W., Dwarakanath, V., Ewing, J., Hirasaki, G.J., Jackson, R.E., Jin, M., Ginn, J.S., Londergan, J.T., Miller, C.A., Pope, G.A., 2002. Performance assessment of NAPL remediation in heterogeneous alluvium. Journal of Contaminant Hydrology 54, 173-193.

Menéndez, B., Zhu, W., Wong, T.-F., 1996. Micromechanics of brittle faulting and cataclastic flow in Berea sandstone. Journal of Structural Geology 18, 1-16.

Morad, M., Nowicka, E., Douthwaite, M., Iqbal, S., Miedziak, P., Edwards, J.K., Brett, G.L., He, Q., Morgan, D., Alshammari, H., Bethell, D., Knight, D.W., Sankar, M., Hutchings, G.J., 2017. Multifunctional supported bimetallic catalysts for a cascade reaction with hydrogen auto transfer: synthesis of 4-phenylbutan-2-ones from 4-methoxybenzyl alcohols. Catal. Sci. Technol. 7, 1928-1936.

Morse, J.W., Arvidson, R.S., 2002. The dissolution kinetics of major sedimentary carbonate minerals. Earth-Sci. Rev. 58, 51-84.

Muller, K., Seubert, A., 2012. Ultra trace determination of fluorobenzoic acids in tap and reservoir water using solid-phase extraction and gas chromatography-mass spectrometry. Journal of chromatography. A 1260, 9-15.

Muller, K., Seubert, A., 2014. Ultra trace determination of fluorobenzoic acids in reservoir and ground water using isotope dilution gas chromatography mass spectrometry. Isotopes in environmental and health studies 50, 277-284. 
Müller, R., Rappert, S., 2010. Pyrazines: occurrence, formation and biodegradation. Applied Microbiology and Biotechnology 85, 13151320.

Nasr, K., Meimoun, J., Favrelle-Huret, A., Winter, J.D., Raquez, J.M., Zinck, P., 2020. Enzymatic Polycondensation of 1,6-Hexanediol and Diethyl Adipate: A Statistical Approach Predicting the Key-Parameters in Solution and in Bulk. Polymers 12, 15.

NPD, 2020. "Facts about oil production". https://www.npd.no/en/facts/production/, accessed on 23.09.2020.

Prado, G.H.C., Rao, Y., de Klerk, A., 2017. Nitrogen Removal from Oil: A Review. Energy \& Fuels 31, 14-36.

Salager, J.-L., Marquez, N., Graciaa, A., Lachaise, J., 2000. Partitioning of Ethoxylated Octylphenol Surfactants in Microemulsion-Oil-Water Systems: Influence of Temperature and Relation between Partitioning Coefficient and Physicochemical Formulation. Langmuir 16, 5534-5539.

Sangster, J., 1989. Octanol-Water Partition Coefficients of Simple Organic Compounds. Journal of Physical and Chemical Reference Data $18,1111-1229$.

Sanni, M., Al-Abbad, M., Kokal, S., Dugstad, Ø., Hartvig, S., Huseby, O., 2018. Pushing the envelope of residual oil measurement: A field case study of a new class of inter-well chemical tracers. Journal of Petroleum Science and Engineering 163, 538-545.

Scandura, G., Palmisano, G., Yurdakal, S., Tek, B.S., Özcan, L., Loddo, V., Augugliaro, V., 2016. Selective photooxidation of ortho-substituted benzyl alcohols and the catalytic role of ortho-methoxybenzaldehyde. Journal of Photochemistry and Photobiology A: Chemistry 328, 122128. 
Schwarzenbach, R.P., Gschwend, P.M., Imboden, D.M., 2002. Partitioning: Molecular Interactions and Thermodynamics, Environmental Organic Chemistry, pp. 57-96.

Serres-Piole, C., Commarieu, A., Garraud, H., Lobinski, R., Preud'homme, H., 2011a. New Passive Water Tracers for Oil Field Applications. Energy \& Fuels 25, 4488-4496.

Serres-Piole, C., Moradi-Tehrani, N., Lobinski, R., Preud'homme, H., 2011b. Direct sensitive simultaneous determination of fluorinated benzoic acids in oil reservoir waters by ultra high-performance liquid chromatography-tandem mass spectrometry. Journal of Chromatography A 1218, 5872-5877.

Serres-Piole, C., Preud'homme, H., Moradi-Tehrani, N., Allanic, C., Jullia, H., Lobinski, R., 2012. Water tracers in oilfield applications: Guidelines. Journal of Petroleum Science and Engineering 98-99, 22-39.

Shen, J., Chen, Y., Wu, S., Wu, H., Liu, X., Sun, X., Li, J., Wang, L., 2015. Enhanced pyridine biodegradation under anoxic condition: The key role of nitrate as the electron acceptor. Chemical Engineering Journal 277, 140-149.

Shook, G.M., Pope, G.A., Asakawa, K., 2009. Determining Reservoir Properties and Flood Performance From Tracer Test Analysis, SPE Annual Technical Conference and Exhibition. Society of Petroleum Engineers, New Orleans, Louisiana, p. 19.

Stephan, C., Dicko, M., Stringari, P., Coquelet, C., 2018. Liquid-liquid equilibria of water + solutes (acetic acid/ acetol/furfural/guaiacol/methanol/phenol/propanal) + solvents (isopropyl acetate/toluene) ternary systems for pyrolysis oil fractionation. Fluid Phase Equilibria 468, 49-57. 
Strand, S., Standnes, D.C., Austad, T., 2006. New wettability test for chalk based on chromatographic separation of $\mathrm{SCN}-$ and SO42-. Journal of Petroleum Science and Engineering 52, 187-197.

Surlyk, F., Stemmerik, L., Ahlborn, M., Harlou, R., Lauridsen, B.W., Rasmussen, S.L., Schovsbo, N., Sheldon, E., Thibault, N., 2010. The cyclic Rordal Member - a new lithostratigraphic unit of chronostratigraphic and palaeoclimatic importance in the upper Maastrichtian of Denmark. Bull. Geol. Soc. Den. 58, 89-98.

Takahashi, K., Hoshino, N., Noro, S.I., Nakamura, T., Akutagawa, T., 2014. A Crystal Structures, Dielectric, and CO2-Adsorption Properties of One-Dimensional $\mathrm{Cu}(\mathrm{II})(2)$ (Adamantane-1-carboxylate)(4)(pyrazine) (infinity) Coordination Polymers with Polar Ligands. Sci. Adv. Mater. 6, $1417-1424$.

Tang, J.S., 1992. Interwell Tracer Tests To Determine Residual Oil Saturation To Waterflood At Judy Creek Bhl'a'pool. PETSOC-91-04-01 $31,12$.

Tang, J.S., 1995. Partitioning Tracers and In-Situ Fluid Saturation Measurements. SPE-22344-PA 10, 33 - 39.

Tang, J.S., 2003. Propagation of phenol in aquifer with reversible adsorption, Proceedings of the Go-Expo Gas and Oil Exposition and the 4 Annual Canadian International Petroleum Conference and the 54 Annual Technical Meeting of the Petroleum Society of CIM : Global Challenges and Technology Integration. Petroleum Society of CIM, Canada.

Tang, J.S., Harker, B., 1991. Interwell Tracer Test To Determine Residual Oil Saturation In A Gas-Saturated Reservoir. Part II: Field Applications. PETSOC-91-04-01 30, 34 - 42.

Tao, X., Zhou, S., Xiang, Z., Ma, J., Hou, R., Zhu, Y., Wei, X., 2017. Fabrication of continuous $\mathrm{ZrB} 2$ nanofibers derived from boron- 
containing polymeric precursors. Journal of Alloys and Compounds 697, 318-325.

Tayyib, D., Al-Qasim, A., Kokal, S., Huseby, O., 2019. Overview of Tracer Applications in Oil and Gas Industry, SPE Kuwait Oil \& Gas Show and Conference. Society of Petroleum Engineers, Mishref, Kuwait, p. 21.

Thomson, B.A., 1998. Atmospheric pressure ionization and liquid chromatography/mass spectrometry-together at last. Journal of the American Society for Mass Spectrometry 9, 187-193.

Valverde-Muñoz, F.J., Seredyuk, M., Muñoz, M.C., Znovjyak, K., Fritsky, I.O., Real, J.A., 2016. Strong Cooperative Spin Crossover in 2D and 3D FeII-MI,II Hofmann-Like Coordination Polymers Based on 2Fluoropyrazine. Inorg. Chem. 55, 10654-10665.

Viig, S.O., Juilla, H., Renouf, P., Kleven, R., Krognes, B., Dugstad, O., Huseby, O.K., 2013. Application of a New Class of Chemical Tracers To Measure Oil Saturation in Partitioning Interwell Tracer Tests, SPE International Symposium on Oilfield Chemistry. Society of Petroleum Engineers, The Woodlands, Texas, USA.

Vorlicek, T.P., Helz, G.R., 2002. Catalysis by mineral surfaces: Implications for Mo geochemistry in anoxic environments. Geochimica et Cosmochimica Acta 66, 3679-3692.

Werle, P., Morawietz, M., Lundmark, S., Sörensen, K., Karvinen, E., Lehtonen, J., 2008. Alcohols, Polyhydric. In Ullmann's Encyclopedia of Industrial Chemistry, (Ed.).

Whitehouse, B.G., 1985. Observation of abnormal solubility behavior of aromatic hydrocarbons in seawater. Marine Chemistry 17, 277-284.

Wood, K.N., Tang, S., Luckasavitch, R.J., 1990. Interwell Residual Oil Saturation at Leduc Miscible Pilot, SPE Annual Technical Conference 
and Exhibition. Society of Petroleum Engineers, New Orleans, Louisiana.

Xie, W.-H., Shiu, W.-Y., Mackay, D., 1997. A review of the effect of salts on the solubility of organic compounds in seawater. Marine Environmental Research 44, 429-444.

Yamada, K., Brousseau, M., Honma, W., Iimura, A., Imase, H., Iwaki, Y., Kawanami, T., LaSala, D., Liang, G., Mitani, H., Nonomura, K., Ohmori, O., Pan, M., Rigel, D.F., Umemura, I., Yasoshima, K., Zhu, G., Mogi, M., 2017. Discovery of a Novel Piperidine-Based Inhibitor of Cholesteryl Ester Transfer Protein (CETP) That Retains Activity in Hypertriglyceridemic Plasma. Journal of Medicinal Chemistry 60, 84668481.

Yu, A.-N., Zhang, A.-D., 2010. The effect of $\mathrm{pH}$ on the formation of aroma compounds produced by heating a model system containing 1ascorbic acid with 1-threonine/l-serine. Food Chemistry 119, 214-219.

Yu, A.-N., Zhou, Y.-Y., Yang, Y.-N., 2017. Kinetics of browning and correlations between browning degree and pyrazine compounds in 1ascorbic acid/acidic amino acid model systems. Food Chemistry 221, 1678-1684.

Yu, W.H., Li, N., Tong, D.S., Zhou, C.H., Lin, C.X., Xu, C.Y., 2013. Adsorption of proteins and nucleic acids on clay minerals and their interactions: A review. Applied Clay Science 80-81, 443-452.

Zhang, J., Wong, T.-F., Davis, D.M., 1990. Micromechanics of pressureinduced grain crushing in porous rocks. Journal of Geophysical Research: Solid Earth 95, 341-352. 
Part 2 



\section{Paper I}

Studies on New Chemical Tracers for Determination of Residual Oil Saturation in the Inter-Well Region.

Mário Silva, Helge Stray, Tor Bjørnstad (2017)

Proceedings of the SPE Oil and Gas Symposium 2017, Oklahoma City, Oklahoma, USA. DOI: 10.2118/185085-MS.

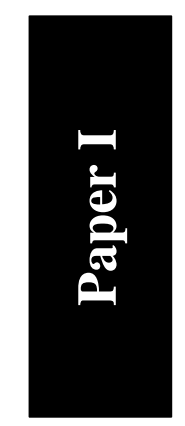


This paper is not included in Brage due to copyright restrictions. 


\section{Paper II}

New Potential Tracer Compounds for Inter-well Sor Determination -

Stability at Reservoir Conditions.

Mário Silva, Helge Stray, Tor Bjørnstad (2017)

Proceedings of the EAGE IOR 2017 - 19th European Symposium on Improved Oil

Recovery, Apr 2017, Stavanger, Norway. DOI: 10.3997/2214-4609.201700351.

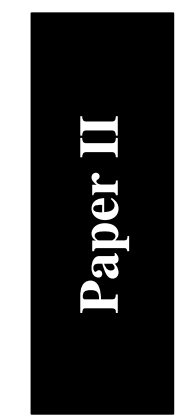


This paper is not included in Brage due to copyright restrictions. 


\section{Paper III}

Stability assessment of PITT tracer candidate compounds: The case of benzyl alcohols.

Mário Silva, Helge Stray, Tor Bjørnstad (2018)

J. Petrol. Sci. Eng., (167) 517 - 523. DOI: 10.1016/j.petrol.2018.04.027.

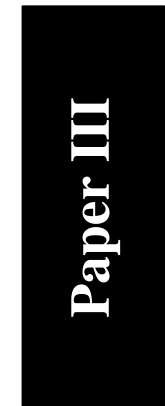




\title{
Stability assessment of PITT tracer candidate compounds: The case of benzyl alcohols
}

\author{
Mario Silva ${ }^{\mathrm{a}, \mathrm{b}, \mathrm{c}, *}$, Helge Stray $^{\mathrm{c}}$, Tor Bjørnstad ${ }^{\mathrm{a}, \mathrm{c}}$ \\ a The National IOR Centre of Norway, University of Stavanger, 4036, Stavanger, Norway \\ b Department of Energy Resources, University of Stavanger, 4036, Stavanger, Norway \\ ${ }^{\mathrm{c}}$ Institute for Energy Technology (IFE), Department of Tracer Technology, Instituttveien 18, 2007, Kjeller, Norway
}

\section{A R T I C L E I N F O}

\section{Keywords:}

Partitioning tracers

Inter-well

Residual oil saturation

Stability

New compounds

\begin{abstract}
A B S T R A C T
The selection of compounds used as inter-well tracers has traditionally been done taking into consideration the goal of the test and the ease in analyzing the chemicals used. This often led to improper selection of the tracers and insufficient knowledge about their behavior under typical reservoir conditions, resulting in several unsuccessful inter-well tracer tests. One of the critical characteristics of any conservative tracer is its stability under various reservoir conditions. In this document we present the study methodology and the findings from stability experiments carried out on 5 benzyl alcohols investigated as oil/water partitioning tracers for partitioning interwell tracer tests (PITT). This is the first of three documents reporting such studies on 3 families of chemicals.

A PITT consists of the simultaneous injection of one or more mass-conservative passive and phase-partitioning (one type of active) tracers. PITT is primarily conceived for mature water-flooded oil fields and measures the residual (or even remaining) oil saturation $\left(\mathrm{S}_{\mathrm{OR}}\right)$ in the swept volumes between wells. Knowledge of $\mathrm{S}_{\mathrm{OR}}$ may be used to identify EOR targets, assess efficiency of EOR operations, and the efficiency of volumetric sweep between wells. This is important information, in particular for the increasing number of mature oil-fields where EOR projects are under consideration and/or development. A thorough knowledge about the static and dynamic properties of the tracers under reservoir conditions is required both to ensure successful field tests, and also to increase accuracy of the data obtained from them.

4-Chlorobenzyl alcohol, 2,6-Dichlorobenzyl alcohol, 4-Methoxybenzyl alcohol, 3,4-Dimethoxybenzyl alcohol, and 4-Hydroxybenzyl alcohol were tested in brine under simulated reservoir conditions with temperatures up to $150{ }^{\circ} \mathrm{C}$ during 12 weeks, at different $\mathrm{pH}$ values, and in the presence of typical sedimentary basin rock materials, sandstone, carbonate rock (limestone), and clay (kaolinite). 4-Chlorobenzyl alcohol and 2,6-Dichlorobenzyl alcohol were found to be fully stable at all tested conditions. 4-Methoxybenzyl alcohol and 3,4-Dimethoxybenzyl alcohol exhibit thermally induced degradation above $125^{\circ} \mathrm{C}$, but may still be considered for use below this temperature. 4-Hydroxybenzyl alcohol exhibits thermally induced degradation which was found to follow a first order kinetics. The apparent activation energy and pre-exponential constant in the Arrhenius law for the degradation reaction were determined and a kinetic model built. This opens the possibility for use of 4Hydroxybenzyl alcohol as a non-conservative reservoir tracer to measure temperature or detect thermal fronts.
\end{abstract}

\section{Introduction}

\subsection{Partitioning inter-well tracer tests and partitioning tracers}

The use of inter-well tracer tests in the oil industry started in the 1960s (Asadi and Shook, 2010; Guan et al., 2005) with molecules successfully applied in hydrology (Ahmad et al., 2003). The future of oil production will increasingly rely on EOR projects to face global demand (IEA, 2016) as the number of mature oil fields increases together with the fact that most of the remaining large unexplored basins rich in hydrocarbons are located in highly environmentally sensitive regions (Muggeridge et al., 2014). Knowledge about residual oil saturation $\left(\mathrm{S}_{\mathrm{OR}}\right)$ in the inter-well region of water flooded reservoirs is a key parameter for the design of EOR operations which can be obtained through a partitioning inter-well tracer test (PITT). PITT in oil reservoirs was introduced by Cooke (1971). The test explores the lag in production experienced by an oil/water partitioning tracer relatively to a passive water tracer. When the partition coefficient $\left(\mathrm{K}=\mathrm{C}_{\mathrm{TR}, \mathrm{O}} / \mathrm{C}_{\mathrm{TR}, \mathrm{W}}\right)$ is known, the delay in production can be directly correlated with the $\mathrm{S}_{\mathrm{OR}}$ in the flow path. Later studies about PITT focus on interpretation of

\footnotetext{
* Corresponding author. The National IOR Centre of Norway, University of Stavanger, 4036, Stavanger, Norway.

E-mail addresses: mario.silva@ife.no, mariohsilva@sapo.pt (M. Silva).
} 
results to increase the test's accuracy and potential gains from the data (Allison et al., 1991; Carpenter, 2016; Huseby et al., 2015; Shen et al., 2017; Tang, 1995) and in reporting the conception and results of field cases (Al-Murayri et al., 2017; Lichtenberger, 1991; Sanni et al., 2016; Tang and Harker, 1991; Viig et al., 2013; Wood et al., 1990). Tritiated small chain alcohols, detectable in very low concentrations using liquid scintillation counting, were commonly used as partitioning tracers until 1990s when environmental regulations starting imposing severe limitations to the use of such compounds (Serres-Piole et al., 2012). After the 1990s these alcohols were still used in their non-radioactive form, however such use lost interest. Propanol and n-butanol were found to be partially adsorbed in the reservoir (Lichtenberger, 1991) and the methods used for their analysis have high detection limits. These factors increase the error on the interpretation of the tracer production curves and the amount of chemicals required for the test. Promising results were reported for 5 fluorinated benzoic acids (Chatzichristos et al., 2000), phenol (Tang, 2003), and Lichtenberger (1991) also tested methyl-ethyl ketone. Information about requirements for selection and testing of PITT tracer candidates can be found in the available literature (Sanni et al., 2016; Serres-Piole et al., 2012; Silva et al., 2017; Viig et al., 2013). To date, only a small number of partitioning tracers for the inter-well region has been developed (Serres-Piole et al., 2012) and there is a need for such new compounds. As for any other type of conservative reservoir tracer, assessing the stability of the partitioning tracer under harsh oilfield conditions is a critical step for its qualification.

In the present work, we report the findings from the stability experiments simulating typical oil reservoir conditions performed on 5 benzyl alcohols. This is the first of 3 documents reporting stability assessment on 3 families of chemicals. Effects of temperature, $\mathrm{pH}$, salinity, and presence of reservoir rock materials were evaluated to obtain a complete description of the behavior and possible use of these chemicals as PITT tracers.

\subsection{Benzyl alcohols}

Benzyl alcohol is an aromatic hydrocarbon naturally occurring in many plants used as solvent and reaction intermediate in chemicalbased industries (Pugh et al., 2015). The chloro-, methoxy- and hydroxyl-substitutes of this compound tested under the scope of the present work exhibit interesting properties for use as PITT tracers, judged from their octanol/water partitioning coefficients $\left(\mathrm{K}_{\mathrm{OW}}\right)$. The relevant properties considered in their selection are presented in Table 1.

It is also important to assess the occurrence of these compounds in oil reservoir fluids or other fluids used in the hydrocarbon recovery process and in the environment, both from natural origin and emitted as pollutants to the hydrosphere from various industries. Large amounts of the studied compounds in the "background" could compromise their use as tracers. Chlorinated, methoxy and hydroxyl benzyl alcohols are not used in large scale industrial operations. Hydroxy, methoxy and chlorobenzyl alcohols are used in small industrial scale as precursors for production of aromatic aldehydes, as model compounds to study the oxidation of substituted benzyl alcohols, and to evaluate the

Table 1

Selected benzyl alcohols partitioning tracer candidates and respective melting point, boiling point, $\mathrm{pKa}$, and octanol/water partitioning coefficient. Data Source: NIST chemistry WebBook

\begin{tabular}{lllll}
\hline Compound & $\begin{array}{l}\text { Melting point } \\
\left({ }^{\circ} \mathrm{C}\right)\end{array}$ & $\begin{array}{l}\text { Boiling point } \\
\left({ }^{\circ} \mathrm{C}\right)\end{array}$ & $\mathrm{K}_{\mathrm{OW}}$ & pKa \\
\hline 4-Hydroxybenzyl alcohol & 114 & 251 & 1,8 & 9,82 \\
4-Chlorobenzyl alcohol & 71 & 232 & 4,2 & 13,9 \\
2,6-Dichlorobenzyl alcohol & 98 & 268 & 7,0 & 13,5 \\
4-Methoxybenzyl alcohol & 23 & 259 & 5,1 & 13,6 \\
3,4-Dimethoxybenzyl alcohol & 22 & 297 & 4,2 & 13,3 \\
\hline
\end{tabular}

performance of selective catalyzed processes aiming for production of aromatic aldehydes (Esteruelas et al., 2011; Higashimoto et al., 2009; Morad et al., 2017; Scandura et al., 2016). 4-Chlorobenzyl alcohols have also been reported to be used as reagent and to assess catalysts for the Friedel-Crafts alkylation of aromatic hydrocarbons (Mantri et al., 2005). 4-Hydroxybenzyl alcohol is also a natural component of scents and flavours used as flavouring and scenting agent in food industry (Jaiswal and Kuhnert, 2014; Martin et al., 2016; Shyamala et al., 2007), in the pharmaceutical industry as an agent to mitigate some neurological disorders (Luo et al., 2017) and in the production of special application ceramic nano-fibers (Tao et al., 2017). This information suggests that reservoir fluids are unlikely to be contaminated with the benzyl alcohols, however any make-up injection water and engineered fluids used in single-well operations should be screened for the possible presence of these tracer candidates before use.

\section{Materials and methods}

\subsection{Materials}

4-Chlorobenzyl alcohol (99\%), 2,6-Dichlorobenzyl alcohol (99\%), 4-Methoxybenzyl alcohol (>98\%), 3,4-Dimethoxybenzyl alcohol (99\%), 4-Hydroxybenzyl alcohol (>98\%), kaolinite (natural aluminium silicate $125 \mu \mathrm{m}-250 \mu \mathrm{m}$ with linear formula $\mathrm{Al}_{2} \mathrm{O}_{3} \cdot 2 \mathrm{SiO}_{2} \cdot 2 \mathrm{H}_{2} \mathrm{O}$ ) and limestone powder type "BCR-116" $\left(95.7 \% \mathrm{CaCO}_{3}+4.3 \% \mathrm{MgCO}_{3}\right)$ were purchased from Sigma-Aldrich (Sigma-Aldrich Norway AS, 0252 Oslo). Berea sandstone powder $(125 \mu \mathrm{m}-250 \mu \mathrm{m})$ was obtained by crushing and sieving sandstone cores purchased from Berea Sandstone ${ }^{\mathrm{TM}}$ Petroleum Cores (Ohio, USA). The composition of the Berea sandstone indicated by the supplier is as follows: $93.13 \%$ silica $\left(\mathrm{SiO}_{2}\right), 3.86 \%$ alumina $\left(\mathrm{Al}_{2} \mathrm{O}_{3}\right), 0.11 \%$ ferric oxide $\left(\mathrm{Fe}_{2} \mathrm{O}_{3}\right), 0.54 \%$ ferrous oxide ( $\mathrm{FeO}), 0.25 \%$ magnesium oxide $(\mathrm{MgO})$, and $0.10 \%$ calcium oxide $(\mathrm{CaO})$.

\subsection{Experimental setup and procedures}

\subsubsection{Preparation of the test tubes}

$50 \mathrm{~cm}$ long durex glass rods (i.d. $6 \mathrm{~mm}$, o. d. $9 \mathrm{~mm}$ ) were cut from $1.5 \mathrm{~m}$ rods (Schott AG, Mitterteich, Germany). The hollow rods were then thermally sealed on one end. The obtained test tubes were submitted to thermal decontamination/healing at $550{ }^{\circ} \mathrm{C}$ during $15 \mathrm{~min}$ under normal atmosphere.

\subsubsection{Preparation of the mixed tracer candidates' solution}

The benzyl alcohols were dissolved in $1 \mathrm{~L}$ of brine further referred to as "synthetic Gullfaks water". Table 2 presents the brine's ionic composition. The solution was then sonicated at room temperature and simultaneously sparged with argon at $5 \mathrm{~mL} / \mathrm{min}$ during $20 \mathrm{~min}$ to remove the dissolved air.

\subsubsection{Stability test procedures}

$2 \mathrm{~mL}$ of the benzyl alcohols solution (2.2.2), kept under constant sparging with argon at $5 \mathrm{~mL} / \mathrm{min}$, were transferred to each tube in four sets of test tubes (2.2.1). One set of test tubes was empty, and the other 3 sets contained $600 \mathrm{mg}$ of Berea sandstone, kaolinite, and limestone, respectively. All test samples were prepared in triplicate. The test tubes containing the samples were then thermally sealed under vacuum. 3 sealed test tubes without any rock material were picked out to determine the exact initial tracer candidates' concentration.

Every heating cabinet had a rotation mechanism incorporated to ensure smooth agitation during the test. These experiments were performed at three different initial $\mathrm{pH}$ values: 5.5, 7.1 and 8.0. The concentration of the benzyl alcohols in the test samples was determined after 1, 3, 6 and 12 weeks of incubation. $\mathrm{pH}$ was also measured at the end of the incubation period. 
Table 2

Composition of the brine (synthetic Gullfaks water) used in the experiments.

\begin{tabular}{|c|c|c|c|c|c|c|c|}
\hline $\mathrm{NaCl}(\mathrm{g} / \mathrm{L})$ & $\mathrm{KCl}(\mathrm{g} / \mathrm{L})$ & $\mathrm{CaCl}_{2} \cdot 2 \mathrm{H}_{2} \mathrm{O}(\mathrm{g} / \mathrm{L})$ & $\mathrm{MgCl}_{2} \cdot 6 \mathrm{H}_{2} \mathrm{O}(\mathrm{g} / \mathrm{L})$ & $\mathrm{BaCl}_{2} \cdot 2 \mathrm{H}_{2} \mathrm{O}(\mathrm{g} / \mathrm{L})$ & $\mathrm{SrCl}_{2} \cdot 6 \mathrm{H}_{2} \mathrm{O}(\mathrm{g} / \mathrm{L})$ & $\mathrm{NaHCO}_{3}(\mathrm{~g} / \mathrm{L})^{\mathrm{a}}$ & $\mathrm{Na}_{2} \mathrm{SO}_{4}(\mathrm{~g} / \mathrm{L})$ \\
\hline 36,855 & 0,629 & 3814 & 2550 & 0,088 & 0437 & 0,157 & 0046 \\
\hline
\end{tabular}

${ }^{\text {a }} \mathrm{NaHCO}_{3}$ was used as $\mathrm{pH}$ buffer in varying amounts $(0,157 \mathrm{~g} / \mathrm{L}$ for initial $\mathrm{pH}$ of 7,1; $0.280 \mathrm{~g} / \mathrm{L}$ for initial $\mathrm{pH}$ of 8,0 ; no $\mathrm{NaHCO}$ for initial $\mathrm{pH}$ of 5,5$)$.

\subsubsection{Quantification of the benzyl alcohols}

The benzyl alcohols were analysed by ultra-performance liquid chromatography (UPLC) with UV detection at $222 \mathrm{~nm}$ and $254 \mathrm{~nm}$. Both the samples used to determine the initial concentration of the solution and the test samples were filtered through a "Chromacol $30 \mathrm{~mm}$ Syringe Filter $0.45 \mu \mathrm{m}$ PTFE" (Thermo Fisher Scientific, Waltham, Massachusetts, United States). $10 \mu \mathrm{L}$ of the filtered test samples were directly injected on an "Agilent Technologies 1290 Infinity II" UPLC (Agilent Technologies, Santa Clara, California, USA) equipped with a Waters "Acquity UPLC BEH" reversed-phase C18 $1.7 \mu \mathrm{m}$ packed column (Waters Corporation, Milford, Massachusetts, USA). The mobile phase consisted of buffer solution of $5 \mathrm{mM} \mathrm{NH} \mathrm{HCO}_{2}$ (A), methanol (B) and acetonitrile (C) at a constant flow of $0.5 \mathrm{~mL} / \mathrm{min}$. Gradient elution was used for separation of the analytes: initially $98 \% \mathrm{~A}$ and $1 \% \mathrm{~B}$. The amount of B increased to $20 \%$ after 4 min and was kept at this value during $1.5 \mathrm{~min}$. Fraction of B was then increased until $70 \%$ at minute 8 and kept at this value during $0.5 \mathrm{~min}$. Gradient conditions were then reset to the initial ones at minute 9 and held during $1 \mathrm{~min}$. The amount of eluent $C$ was constant at $1 \%$ during the $10 \mathrm{~min}$ chromatographic run.

\subsection{Degradation kinetics}

Treatment and analysis of the experimental data for one of the tracer candidates was performed in order to investigate the possibility of its use as a non-conservative reservoir tracer. The method of time, based on the integrated rate law, was applied to the analysis of the degradation kinetics of 4-Hydroxybenzyl alcohol. The mass balance of a given compound A in a closed system can be written as (Fogler, 2006):

$K^{\prime} C_{A}^{n}=-\frac{d C_{A}}{d t}$

where $\mathrm{C}_{\mathrm{A}}$ is the concentration of compound $\mathrm{A}, \mathrm{K}^{\prime}$ is the apparent degradation rate constant and $\mathrm{n}$ the order of the reaction. The data used was obtained from experiments performed in brine, thus the use of the designation "apparent rate constant". The salinity of the experimental aqueous medium could influence the rate of the reaction. By integrating equation (1) supposing the reaction follows a first-order kinetics:

$\ln \left(\frac{C_{A}}{C_{A 0}}\right)=-K^{\prime} t$

where $\mathrm{C}_{\mathrm{A} 0}$ is the initial concentration of compound $\mathrm{A} . \mathrm{K}^{\prime}$ is temperature dependent. Through linear regression analysis of the results at the different tested temperatures, K' (T) can be obtained. The apparent activation energy of the reaction and the pre-exponential factor of the rate constant can be obtained from the linearized Arrhenius equation:

$\ln K(T)=\ln A-\frac{E_{a}}{R} \frac{1}{T}$

where $\mathrm{A}$ is the pre-exponential factor, $\mathrm{E}_{\mathrm{a}}$ the apparent activation energy, $R$ the gas constant, and $\mathrm{T}$ the temperature in Kelvin (K).

\section{Results and discussion}

\subsection{Influence of $p H$}

Fakhru'l-Razi et al. (2009) present extensive data on physicochemical characteristics of produced waters from oil reservoirs. The initial
$\mathrm{pH}$ values in the stability experiments were intended to cover a $\mathrm{pH}$ range representative of real conditions. The $\mathrm{pH}$ value remained stable until the end of the experimental thermal incubation period. The largest measured variations were a decrease of 0.3 units in the experiments with kaolinite as substrate after 12 weeks at initial $\mathrm{pH} 7.1$, increase of 0.2 units in the experiments with Berea sandstone as substrate after 12 weeks at initial $\mathrm{pH} 5.0$, and an increase of 0.4 units in the experiments with limestone as substrate after 12 weeks at initial $\mathrm{pH}$ 5.0. No significant variation on the $\mathrm{pH}$ of the stability experiments carried out without rock substrate was observed. Results suggest that $\mathrm{pH}$ has no impact on the stability of the benzyl alcohols. The concentration of the tracer candidates in samples from experiments with the same time and temperature of incubation does not vary at all tested $\mathrm{pH}$ values. Complete results may be found in Figs. A1 - A4 and B1 - B4 in the "supplementary materials" section to this document.

\subsection{Influence of temperature and time}

The remaining fraction of the tracer candidates (RF) is defined as the concentration as a function of temperature and time of incubation of the compounds divided by their initial concentration. For a fully stable tracer candidate, a RF equal to 1 , considering the uncertainty of the analytical method used for its quantification, should be observed under all experimental conditions. The results allowed organizing the tracer candidates in 3 groups: fully stable, partially stable and unstable with other potential applications.

4-Chlorobenzyl alcohol (4-Cl-BZA) and 2,6-Dichlorobenzyl alcohol (2,6-DiCl-BZA) exhibit no decrease in concentration after the total incubation period at every tested temperature. Fig. 1 presents RF values for 4-Cl-BZA and 2,6-DiCl-BZA as a function of time of incubation at all tested temperatures in the experiments at initial $\mathrm{pH}$ of 7.1. Both the compounds possess the required stability for use as active conservative tracers in oil reservoirs. Results for 4-Methoxybenzyl alcohol (4-METBZA) and 3,4-Dimethoxybenzyl alcohol (3,4-DiMET-BZA) are shown in Fig. 2.

Both these compounds exhibit a very high stability within temperatures up to $125^{\circ} \mathrm{C}$ during the total period of testing. Loss in $\mathrm{RF}$ is observed at $150^{\circ} \mathrm{C}$ both for 4-MET-BZA and 3,4-DiMET-BZA during the whole period of incubation. A trend is observable as function of time at this temperature; the amount lost increases as the time of incubation increases. Considering that such behavior was not observed at all other temperatures, the observed decrease of $\mathrm{RF}$ at $150^{\circ} \mathrm{C}$ is most likely reflecting a purely temperature dependent mechanism: the energy of the system reaches the necessary value to trigger degradation and/or reaction of the compounds above $125^{\circ} \mathrm{C}$.

Attempts to identify degradation products which would allow proposing a reaction mechanism to validate these assumptions have proven unsuccessful.

Many oilfields have temperatures below $125^{\circ} \mathrm{C}$, therefore 4-METBZA and 3,4-DiMET-BZA may still be considered for use as active conservative reservoir tracers. The onset of degradation may even be exploited to detect thermal fronts in the swept volumes of the reservoirs. Results indicate that if a significant loss of 4-MET-BZA or 3,4DiMET-BZA is observed in a real field test, it will most likely be caused by a thermal effect. At $150{ }^{\circ} \mathrm{C}$, a loss of about $25 \%$ was observed just after 1 week. The velocities of fluids inside a reservoir are low (typically around 1 foot/day), which lead to significant contact periods between the tracers and existing thermal fronts. This would result in a 

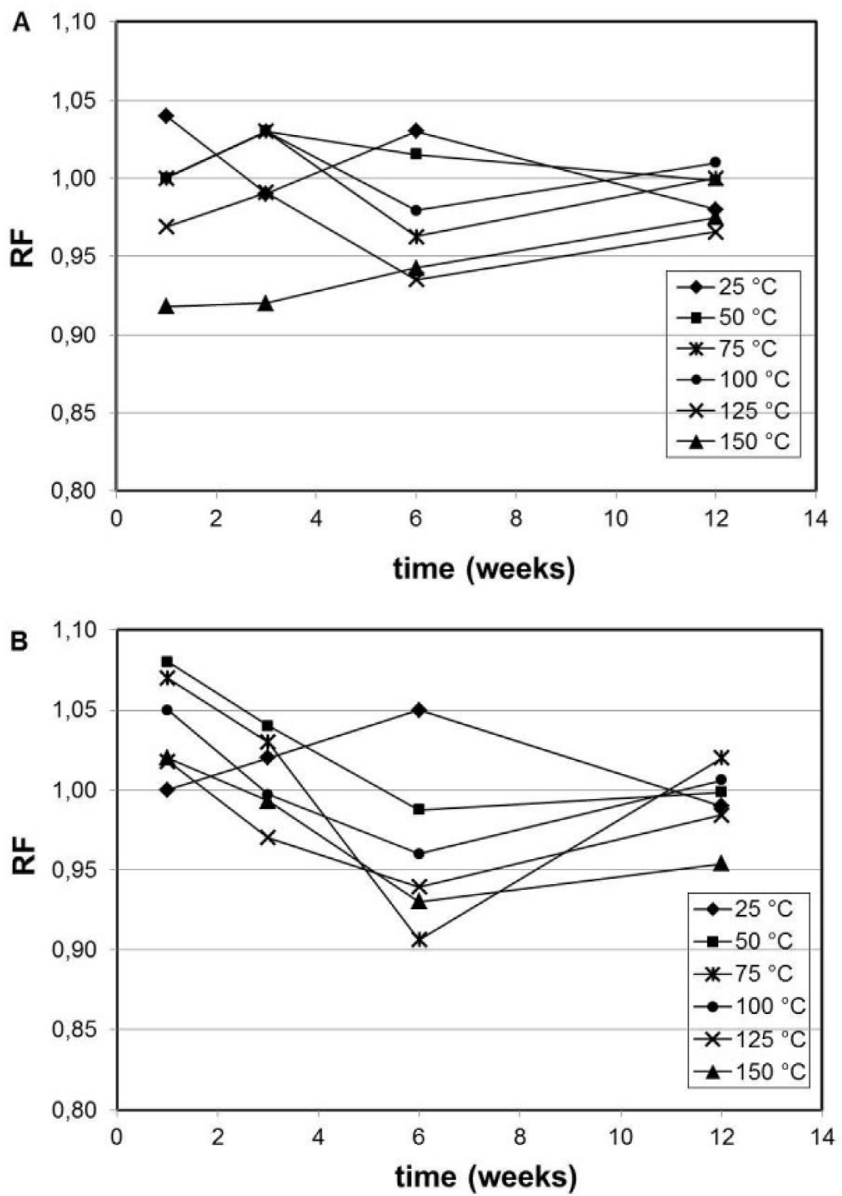

Fig. 1. RF of 4-Chlorobenzyl alcohol and 2,6-Dichlorobenzyl alcohol as function of time and temperature of incubation. (A) 4-Chlorobenzyl alcohol, (B) 2,6Dichlorobenzyl alcohol. Initial experimental $\mathrm{pH}$ 7.1, no rock substrate).

considerable loss of the original tracer compound, thus allowing detection of the temperature variation. Such information is important, particularly if EOR methods are under consideration or use, as they are likely to be temperature dependant.

4-Hydroxybenzyl alcohol (4-HYD-BZA) exhibits high stability during 12 weeks at the lowest tested temperatures $\left(25^{\circ} \mathrm{C}\right.$ and $\left.50^{\circ} \mathrm{C}\right)$. This compound degrades at temperatures $\geq 75^{\circ} \mathrm{C}$. These results also suggest a thermally driven phenomenon where increasing temperature leads to the available energy of the system to rise above the activation energy barrier of the degradation reaction. Fig. 3 presents 4-HYD-BZA's $\mathrm{RF}$ as a function of time and temperature in the experiments at initial $\mathrm{pH}$ of 7.1 without any rock substrate.

The rate of degradation of 4-HYD-BZA is clearly temperature dependant, increasing with the increase of $\mathrm{T}$ (lowest values for RF are achieved faster as $\mathrm{T}$ increases). The decrease in the RF is not linear at any tested temperature which suggests that the degradation kinetics is concentration dependant. A linear decrease in the RF of the compound should be expected if the order of reaction was zero relatively to the concentration of 4-HYD-BZA. Further treatment of the experimental data relative to this compound was performed to assess the possibility of its use as a non-conservative active reservoir tracer to retrieve information about temperature in flooded areas of an oil reservoir.

\subsection{Influence of rock substrate}

For a compound to be used as a conservative tracer in an oil reservoir it is crucial that it has no significant interaction with the rocks that form the porous medium, which could affect both its stability and
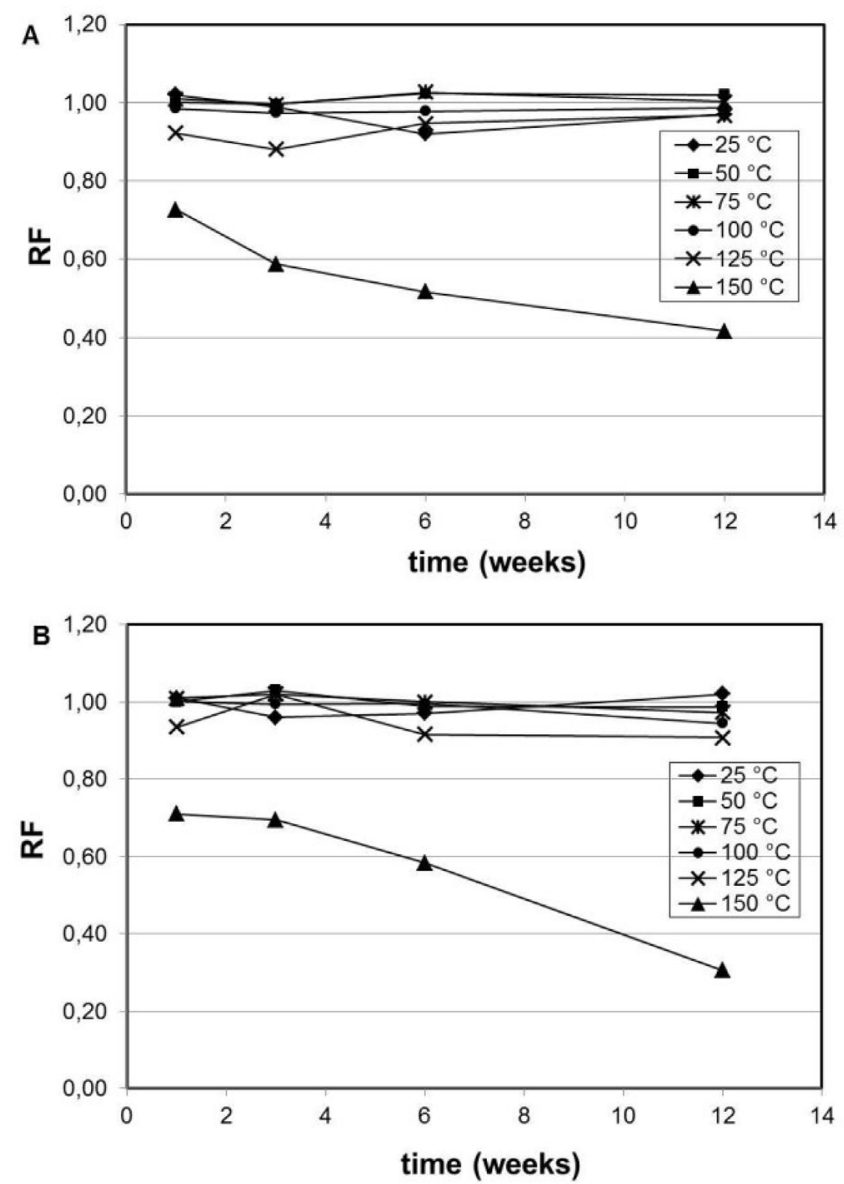

Fig. 2. RF of 4-Methoxybenzyl alcohol and 3,4-Dimethoxybenzyl alcohol as function of time and temperature of incubation. (A) 4-Methoxybenzyl alcohol, (B) 3,4-Dimethoxybenzyl alcohol. Initial experimental $\mathrm{pH}$ 7.1, no rock substrate).

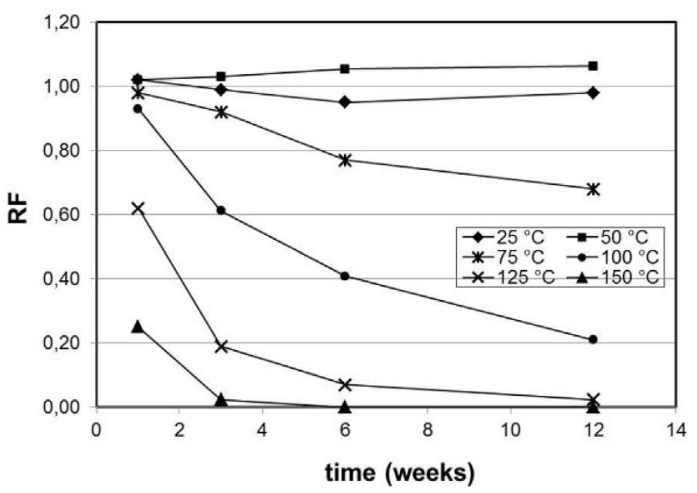

Fig. 3. RF of 4-Hydroxybenzyl alcohol as function of time and temperature of incubation. Initial experimental $\mathrm{pH} 7.1$, no rock substrate.

dynamic properties. Conventional sedimentary oil basins are primarily constituted by sandstones and/or carbonates which present a significant variation in geochemistry from reservoir to reservoir and even within the same formation (Carroll et al., 2016; Morad et al., 2010). Thus selecting a rock substrate completely representative of every possible formation encountered to test the interaction of the tracer candidates with it is an impossible task. Clays are also present in the rock formations with kaolinite being one of the most occurring (Jiang, 2012). Kaolinite has been reported to be a good adsorbent both for ions (Bhattacharyya and Gupta, 2008) and organic compounds (Yu et al., 


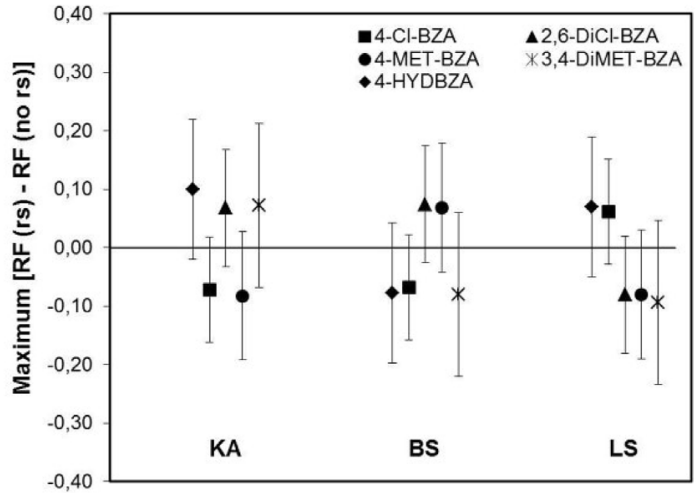

Fig. 4. Maximum differences encountered in RF values between experiments with rock substrate (rs) and experiments without rock substrate $\pm 2 \times \sigma$ in RF (t, T). (KA) kaolinite, (BS) Berea sandstone, (LS) limestone.

2013). Often ion exchange is the proposed adsorption mechanism. Berea sandstone, limestone and kaolinite were selected as "model" reservoir rock substrates to assess the behavior of the tracer candidates when in contact with sedimentary oil basins.

The RF ( $t, T$ ) for each benzyl alcohol obtained from the stability experiments using each of the 3 rock substrates were compared with the respective $R F(t, T)$ in the stability experiments without any rock substrate. Fig. 4 presents the maximum absolute difference encountered between the RFs for each compound and rock substrate. Differences are presented together with 2 times the standard deviation associated with the determination of the RF value from the tests performed in triplicate. No significant difference was observed between RF at a given time and temperature of incubation in the experiments with rock substrate and the corresponding experiments without rock substrate. Even the highest differences encountered fall inside the uncertainty in the determination of the compounds' concentration. No systematic trend is also observable regarding the benzyl alcohols or the rock substrates. Results suggest that $\mathrm{RF}$ values obtained in the presence of the model reservoir rocks are randomly higher or lower than the ones in experiments without any rock material. This apparent lack of interaction between the benzyl alcohols and typical reservoir rocks meets one of the requirements for their use as tracers in oil reservoirs.

\subsection{Kinetics of degradation of 4-hydroxybenzyl alcohol}

A study on the degradation of 4-HYD-BZA was performed to assess its use as a tracer to measure temperature in the flooded areas (preferably short sections) of an oil reservoir, or to detect thermal fronts which may be present in lower temperature fields. Such use requires knowledge of the reaction of degradation's activation energy and the pre-exponential factor of the Arrhenius equation. These parameters can be obtained from the treatment of the data from the stability experiments. A first order reaction was assumed for data treatment. Experimental data at $75^{\circ} \mathrm{C}, 100^{\circ} \mathrm{C}, 125^{\circ} \mathrm{C}$, and $150{ }^{\circ} \mathrm{C}$ were used. Values of $C_{A} / C_{A 0}(R F)$ at time 0 (when $C_{A} / C_{A 0}=R F=1$ in every used temperature) were included to increase the number of points in the linear regression analysis. Fig. 5 presents the natural logarithm of RF as a function of time (equation (2)) at the considered temperatures. The slopes in the linear regressions directly supply the rate constant of the reaction $(\mathrm{K})\left(\right.$ weeks $^{-1}$ ) for each temperature.

The $\ln (\mathrm{K}(\mathrm{T})$ ) values were then plotted against $1 / \mathrm{T}(\mathrm{K})$ and a linear regression analysis was performed. Fig. 6 presents the plot. From the linearized form of the Arrhenius equation (eq. (3)) follows that the slope equals $E_{a} / R$ and $\ln (A)$ is the y-intercept. $E_{a}$ has a value $58,8 \mathrm{~kJ} /$ mol and $\mathrm{A}=2,30 \mathrm{E}+07$ weeks $^{-1}$. Equation (2) can be rewritten as follows with the Arrhenius parameters:

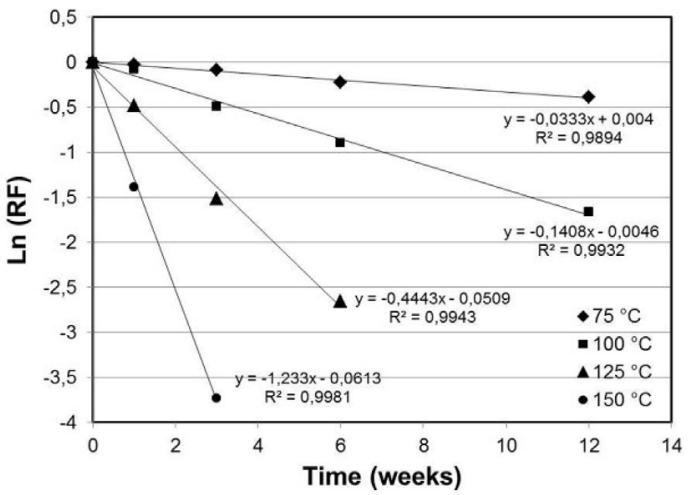

Fig. 5. Ln (RF) of 4-hydroxybenzyl alcohol as a function of time and linear regressions at each temperature. Initial experimental $\mathrm{pH} 7.1$, no rock substrate.

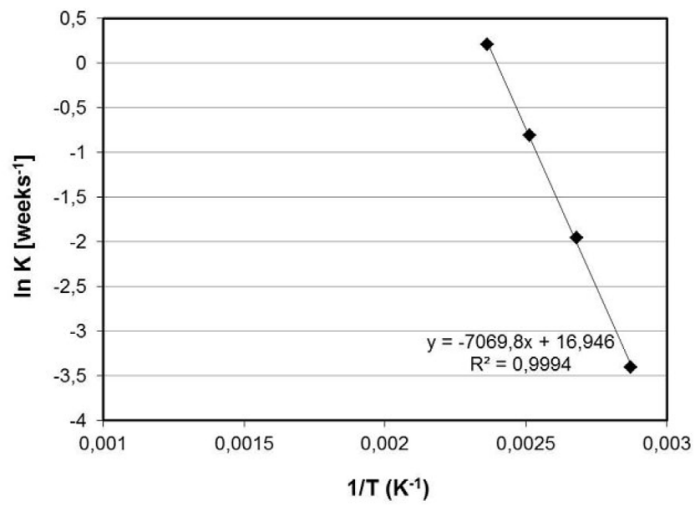

Fig. 6. Arrhenius plot for the degradation of 4-HYD-BZA between 75 and $150^{\circ} \mathrm{C}$.

$\ln \left(\frac{C_{A}}{C_{A 0}}\right)=-2,30 \times 10^{07} e^{\frac{-58,8 \times 10^{03}}{R T}} \cdot t$

Equation (4) is the model which describes RF of 4-HYD-BZA as a function of temperature and time, which can be used to obtain information about temperature in a reservoir.

The values of the RF of 4-HYD-BZA predicted by equation (4) were compared with the experimental ones to assess the accuracy of the model. 2 additional tests (2.2.3) were performed where RF values were determined after 2 and 4 weeks. Fig. 7 presents the experimental RF values, $\pm 2 \sigma$ in the determination of each one, vs the RF values predicted by equation (4) for 4-HYD-BZA, between 75 and $150{ }^{\circ} \mathrm{C}$.

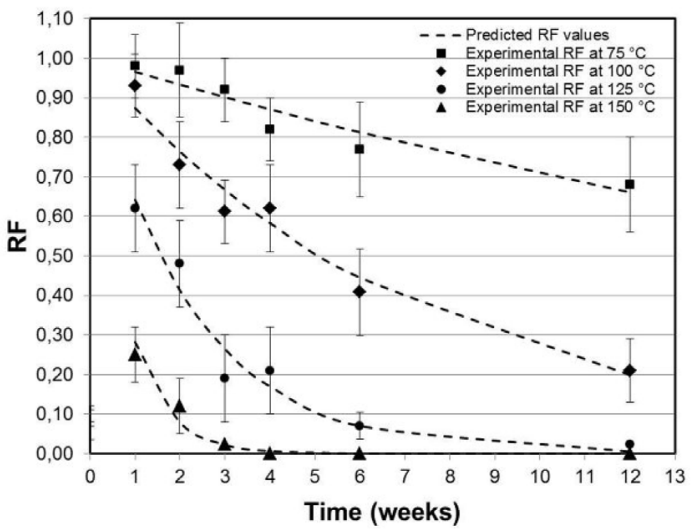

Fig. 7. Experimental vs predicted RF values of 4-HYD-BZA between 75 and $150{ }^{\circ} \mathrm{C}$. Uncertainty is not depicted for values under the lower analytical threshold $(\mathrm{RF}=0,05)$. 
Good agreement is encountered between experimental and predicted data. Predicted values are always included in the confidence interval accounting for the variance in the experimental determinations. This suggests the assumption made of a first order kinetics of degradation is a sufficiently accurate description of the phenomena, and that no other chemical species present in the bulk is taking part in the reaction.

The use of non-conservative tracers in an oil reservoir is not common, but can provide useful information if the tracer's degradation process is understood and/or modellable. Chemical EOR processes such as conformity control with micro-emulsions, permeability modification with gels, or wettability modification with surfactants, for example, depend strongly on temperature. Thus previous up-front knowledge of this in-situ parameter can be valuable to increase recovery rates and decrease operational cost.

\section{Conclusions}

The stability of five benzyl alcohols was investigated in batch experiments under typical conditions encountered in oil reservoirs as part of their qualification process for use as partitioning tracers (one type of active tracers). Experiments were performed in a synthetic brine to account for the effect of elevated salinity (ion strength and ionic composition). The influence of temperature, time, and $\mathrm{pH}$ on the stability of the compounds was evaluated. Interaction with typical reservoir rock (kaolinite, sandstone and limestone) was also studied. The $\mathrm{pH}$ was found to have no significant impact on the stability of all tested compounds in the studied range $(5,5-8,0)$. No difference outside the analytical uncertainty was encountered between experiments at different $\mathrm{pH}$ values. 4-Chlorobenzyl alcohol and 2,6-Dichlorobenzyl alcohol were found to be fully stable up to $150^{\circ} \mathrm{C}$ during 12 weeks. No significant interaction between these 2 compounds and the rock materials was observed. 4-Methoxybenzyl alcohol and 3,4-Dimethoxybenzyl alcohol are also insensitive to the presence of rock substrates. These compounds were found to exhibit limited thermal stability. Both are fully stable up to $125^{\circ} \mathrm{C}$ and 12 weeks, and an onset of degradation is observable at $150^{\circ} \mathrm{C}$ even after 1 week of incubation. 4-Methoxybenzyl alcohol and 3,4-Dimethoxybenzyl alcohol may still be used as partitioning tracers, as most oil reservoirs have temperatures below $125^{\circ} \mathrm{C}$. The degradation they exhibit above $125^{\circ} \mathrm{C}$ may also be exploited to retrieve information about the existence of thermal fronts in the swept volumes of oil fields. 4-Hydroxybenzyl alcohol is stable during the 12 weeks of testing at 25 and $50^{\circ} \mathrm{C}$ exhibiting degradation at temperatures above this threshold. The degradation of this compound increases both with temperature and time of incubation, and is not affected by the presence of rock materials. Results agree with a first order degradation kinetic purely thermally driven without influence of any other chemical species. The apparent activation energy and the pre-exponential factor of the Arrhenius equation were determined and a kinetic model for the degradation was developed, thus opening the possibility for using this compound as a non-conservative active (temperature sensitive) tracer.

\section{Acknowledgements}

The authors acknowledge the Research Council of Norway (230303) and the industry partners; ConocoPhillips Skandinavia AS, Aker BP ASA, Eni Norge AS, Maersk Oil Norway AS, Statoil Petroleum AS, ENGIE E\&P Norge AS, Lundin Norway AS, Halliburton AS, Schlumberger Norge AS, Wintershall Norge AS, DEA Norge AS of The National IOR Centre of Norway for support.

\section{Appendix A. Supplementary data}

Supplementary data related to this article can be found at http://dx. doi.org/10.1016/j.petrol.2018.04.027.

\section{References}

Ahmad, M., et al., 2003. Interwell tracing by environmental isotopes at Fimkassar Oilfield, Pakistan. Applied radiation and isotopes: including data, instrumentation and methods for use in agriculture. industry Med. 58 (5), 611-619.

Al-Murayri, M.T., Al-Qenae, A., AlRukaibi, D., Chatterjee, M., Hewitt, P., 2017. SPE187549-MS In: Proceedings of the SPE Kuwait Oil\&Gas Show and Conference, Kuwait 15-18 October, 2017. Society of Petroleum Engineers.

Allison, S.B., Pope, G.A., Sepehrnoori, K., 1991. Analysis of field tracers for reservoir description. J. Petroleum Sci. Eng. 5 (2), 173-186.

Asadi, M., Shook, G.M., 2010. SPE-126029-MS In: Proceedings of the SPE North Africa Technical Exhibition, Cairo, Egypt, 14-17 February 2010. Society of Petroleum Engineers.

Bhattacharyya, K.G., Gupta, S.S., 2008. Adsorption of a few heavy metals on natural and modified kaolinite and montmorillonite: a review. Adv. Colloid Interface Sci. 140 (2), $114-131$.

Carpenter, C., 2016. Use of partitioning tracers to estimate oil-saturation distribution. J. Petroleum Technol. 68 (09), 72-73.

Carroll, S., et al., 2016. Review: role of chemistry, mechanics, and transport on well integrity in CO2 storage environments. Int. J. Greenh. Gas Control 49, 149-160.

Chatzichristos, C., Dugstad, Ø., Haugan, A., Sagen, J., Muller, J., 2000. SPE 59369 In: Proceedings of the 2000 SPE/DOE Improved Oil Recovery Symposium, Tulsa,Oklahoma, 3-5 April 2000. Society of Petroleum Engineers.

Cooke, C.E.J., 1971. Method of Determining Fluid Saturations in Reservoirs. Esso Production Research Company, USA.

Esteruelas, M.A., García-Obregón, T., Herrero, J., Oliván, M., 2011. Osmium-catalyzed oxidation of primary alcohols with molecular oxygen. Organometallics 30 (23), 6402-6407.

Fakhru'l-Razi, A., et al., 2009. Review of technologies for oil and gas produced water treatment. J. Hazard. Mater. 170 (2), 530-551.

Fogler, H.S., 2006. Elements of Chemical Reaction Engineering. Prentice Hall PTR, New Jersey.

Guan, L., Du, Y., Johnson, S.G., Choudhary, M.K., 2005. Advances of Interwell Tracer Analysis in the Petroleum Industry.

Higashimoto, S., et al., 2009. Selective photocatalytic oxidation of benzyl alcohol and its derivatives into corresponding aldehydes by molecular oxygen on titanium dioxide under visible light irradiation. J. Catal. 266 (2), 279-285.

Huseby, O., Hartvig, S.K., Jevanord, K., Dugstad, Ø., 2015. Assessing EOR potential from partitioning tracer data. In: SPE Middle East Oil \& Gas Show and Conference. Society of Petroleum Engineers, Manama, Bahrain.

IEA, 2016. Key World Energy Statistics 2016. IEA, Paris, pp. 80.

Jaiswal, R., Kuhnert, N., 2014. Identification and characterization of the phenolic glycosides of lagenaria siceraria stand. (Bottle gourd) fruit by liquid chromatographytandem mass spectrometry. J. Agric. Food Chem. 62 (6), 1261-1271.

Jiang, S., 2012. Clay minerals from the perspective of oil and gas exploration. In: Valaškova, M., Martynkova, G.S. (Eds.), Clay Minerals in Nature - Their Characterization, Modification and Application. InTech, Rijeka pp. Ch. 2.

Lichtenberger, G.J., 1991. Field applications of interwell tracers for reservoir characterization of enhanced oil recovery pilot areas. In: SPE Production Operations Symposium. Society of Petroleum Engineers, Oklahoma City, Oklahoma.

Luo, L., et al., 2017. Anti-oxidative effects of 4-hydroxybenzyl alcohol in astrocytes confer protective effects in autocrine and paracrine manners. PLoS One 12 (5) e0177322.

Mantri, K., Komura, K., Kubota, Y., Sugi, Y., 2005. Friedel-Crafts alkylation of aromatics with benzyl alcohols catalyzed by rare earth metal triflates supported on MCM-41 mesoporous silica. J. Mol. Catal. A Chem. 236 (1), 168-175.

Martin, V., et al., 2016. De novo synthesis of benzenoid compounds by the yeast hanseniaspora vineae increases the flavor diversity of wines. J. Agric. Food Chem. 64 (22), 4574-4583.

Morad, M., et al., 2017. Multifunctional supported bimetallic catalysts for a cascade reaction with hydrogen auto transfer: synthesis of 4-phenylbutan-2-ones from 4methoxybenzyl alcohols. Catal. Sci. Technol. 7 (9), 1928-1936.

Morad, S., Al-Ramadan, K., Ketzer, J.M., De Ros, L.F., 2010. The impact of diagenesis on the heterogeneity of sandstone reservoirs: a review of the role of depositional facies and sequence stratigraphy. AAPG Bull. 94 (8), 1267-1309.

Muggeridge, A., et al., 2014. Recovery rates, enhanced oil recovery and technological limits. Philosophical transactions. Series A. Math. Phys. Eng. Sci. 372 (2006) 20120320.

Pugh, S., McKenna, R., Halloum, I., Nielsen, D.R., 2015. Engineering Escherichia coli for renewable benzyl alcohol production. Metab. Eng. Commun. 2, 39-45.

Sanni, M., et al., 2016. SPE-181324-MS In: Proceedings of the SPE Annual Technical Conference and Exhibition held in Dubai, UAE, 26-28 September 2016. Society of Petroleum Engineers.

Scandura, G., et al., 2016. Selective photooxidation of ortho-substituted benzyl alcohols and the catalytic role of ortho-methoxybenzaldehyde. J. Photochem. Photobiol. A Chem. 328, 122-128.

Serres-Piole, C., et al., 2012. Water tracers in oilfield applications: Guidelines. J. Petroleum Sci. Eng. 98-99 (Suppl. C), 22-39.

Shen, T., Moghanloo, R.G., Tian, W., 2017. SPE-187400-MS In: Proceedings of the SPE Annual Technical Conference and Exhibition held in San Antonio, Texas, USA, 9-11 October 2017. Society of Petroleum Engineers.

Shyamala, B.N., Naidu, M.M., Sulochanamma, G., Srinivas, P., 2007. Studies on the antioxidant activities of natural vanilla extract and its constituent compounds through in vitro models. J. Agric. Food Chem. 55 (19), 7738-7743.

Silva, M., Stray, H., Bjørnstad, T., 2017. SPE-185085 In: Proceedings of the SPE Oklahoma City Oil and Gas Symposium held in Oklahoma City, Oklahoma, USA, 
27-30 March 2017. Society of Petroleum Engineers.

Tang, J.S., 1995. Partitioning tracers and in-situ fluid saturation measurements. SPE Form. Eval. 10 (01), 33-39.

Tang, J.S., 2003. Propagation of phenol in aquifer with reversible adsorption. In: Proceedings of the Go-expo Gas and Oil Exposition and the 4 Annual Canadian International Petroleum Conference and the 54 Annual Technical Meeting of the Petroleum Society of CIM : Global Challenges and Technology Integration. Petroleum Society of CIM, Canada.

Tang, J.S., Harker, B., 1991. Interwell tracer test to determine residual oil saturation in a gas-saturated reservoir. Part II: field applications. J. Can. Petroleum Technol. 30 (04), $34-42$
Tao, X., et al., 2017. Fabrication of continuous ZrB2 nanofibers derived from boroncontaining polymeric precursors. J. Alloys Compd. 697, 318-325.

Viig, S.O., et al., 2013. Application of a new class of chemical tracers to measure oil saturation in partitioning interwell tracer tests. In: SPE International Symposium on Oilfield Chemistry. Society of Petroleum Engineers. The Woodlands, Texas, USA.

Wood, K.N., Tang, S., Luckasavitch, R.J., 1990. Interwell residual oil saturation at leduc miscible pilot. In: SPE Annual Technical Conference and Exhibition. Society of Petroleum Engineers, New Orleans, Louisiana.

Yu, W.H., et al., 2013. Adsorption of proteins and nucleic acids on clay minerals and their interactions: a review. Appl. Clay Sci. 80-81, 443-452. 



\section{Paper IV}

Stability assessment of PITT tracer candidate compounds - The case of pyrazines.

Mário Silva, Helge Stray, Tor Bjørnstad (2019)

J. Petrol. Sci. Eng., (182) 106269. DOI: 10.1016/j.petrol.2019.106269.

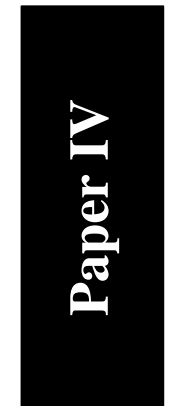




\title{
Stability assessment of PITT tracer candidate compounds - The case of pyrazines
}

\author{
Mario Silva ${ }^{\mathrm{a}, \mathrm{b}, \mathrm{c}, *}$, Helge Stray ${ }^{\mathrm{c}}$, Tor Bjørnstad ${ }^{\mathrm{a}, \mathrm{c}}$ \\ ${ }^{a}$ The National IOR Centre of Norway, University of Stavanger, 4036 Stavanger, Norway \\ ${ }^{\mathrm{b}}$ Department of Energy Resources, University of Stavanger, 4036 Stavanger, Norway \\ ${ }^{\mathrm{c}}$ Institute for Energy Technology (IFE), Department of Tracer Technology, Instituttveien 18, 2007 Kjeller, Norway
}

\section{A R T I C L E I N F O}

\section{Keywords:}

Partitioning tracers

Inter-well

New compounds

Pyrazines

Stability

Residual oil saturation

\begin{abstract}
A B S T R A C T
The determination of the residual oil saturation $\left(\mathrm{S}_{\mathrm{OR}}\right)$ in the volumes swept between injector/producer well pairs is a parameter of major importance for the design and/or evaluation of IOR projects. The number of IOR projects is increasing with the growing number of mature oilfields. The partitioning inter-well tracer test (PITT) is used to measure $\mathrm{S}_{\mathrm{OR}}$ in waterflooded reservoirs and relies on the use of partitioning and passive tracers. Many PITT were unsuccessful in the past due to a poor knowledge about the compounds used as tracers and to date, very few compounds were developed for this application. New partitioning tracers are needed, and a systematic qualification process is necessary to reduce the risk of costly unsuccessful field tests. In the present document we report and discuss the findings from stability experiments performed on 2 alkylpyrazines, 1 methoxypyrazine, and 2 halogenated pyrazines under investigation for use as oil/water partitioning tracers for determination of the $\mathrm{S}_{\mathrm{OR}}$ in the inter-well region of water flooded oil reservoirs.

The stability of 2,3-dimethylpyrazine, 2,6-dimethylpyrazine, 2-methoxypyrazine, 2-chloropyrazine, and 2fluoropyrazine in brine solutions was evaluated in batch experiments at temperatures ranging from $25^{\circ} \mathrm{C}$ to $150{ }^{\circ} \mathrm{C}$ for 12 weeks, at 3 different $\mathrm{pH}$ values and in the presence of typical oil reservoir rock materials (sandstone, carbonate rock, and clay). Results suggest that 2,3-dimethylpyrazine and 2,6-dimethylpyrazine possess the required stability and absence of significant interaction with the rock substrates. 2-chloropyrazine and 2fluoropyrazine degrade with a temperature and $\mathrm{pH}$ dependent rate but are not influenced by the rock materials. 2-methoxypyrazine exhibits temperature dependent degradation in the absence of any rock substrate and in the presence of sandstone and carbonate rock. A strong interaction was observed between this compound and the clay material (kaolinite) which is responsible for dramatically increasing its degradation rate.
\end{abstract}

\section{Introduction}

\subsection{Partitioning inter-well tracer tests}

The basic principle behind a partitioning inter-well tracer test (PITT) is that an oil/water partitioning tracer will exhibit a constant and reversible equilibrium distribution between a hydrocarbon phase and an aqueous phase (Cooke, 1971; Leo et al., 1971). When flowing through a porous medium containing a stagnant (or nearly stagnant) non-aqueous phase, such equilibrium distribution will cause a delay in the production of the partitioning tracer relatively to a passive water tracer. This delay can be explored to determine the concentration and distribution of the hydrocarbon phase. PITT was first used in hydrogeology to evaluate subsoil contamination by non-aqueous phase liquids and the effect of decontamination operations. It is an important method to retrieve information about the subsoil within this scientific field (Jin et al., 1995; McDonald et al., 2016; Wang et al., 2013).

The learnings from the use of PITT in hydrogeology were useful for introducing the technique to the oil industry in 1971 (Cooke, 1971) and provided valuable insight for the development of such tests in oilfields. Nevertheless, partitioning tracers in the oilfields typically face very harsh conditions for long periods of time (months or even years), which bring far more challenges to the used molecules than the conditions encountered in near ground surface NAPL contaminated sites (Silva et al., 2017). Thus, the use of a new compound as partitioning tracer in the oilfield must be preceded by an evaluation of its capacity to resist the conditions encountered there. The potential benefits of using PITT as a characterization tool for efficient reservoir management are widely discussed in the literature (Al-Murayri et al., 2017; Dean et al., 2016; Huseby et al., 2015; Sanni et al., 2018; Silva et al., 2017) and such tests

\footnotetext{
* Corresponding author. The National IOR Centre of Norway, University of Stavanger, 4036 Stavanger, Norway.

E-mail addresses: mario.silva@ife.no, mariohsilva@sapo.pt (M. Silva).
} 
can be fundamental for the industry in the future. Increasing the number of compounds tested and thoroughly qualified for use as partitioning tracers in the oilfield will contribute to overcome one of the main current obstacles to the dissemination of this technology.

Our findings from the laboratory testing under simulated oilfield conditions of 5 pyrazines under study as partitioning tracer candidates are presented in the current document. This is the second of 3 documents on the stability assessment of chemical compounds from 3 different chemical families. The experiments carried out considered the possible effects of temperature, $\mathrm{pH}$, salinity, and presence of reservoir rock materials.

\subsection{Pyrazines}

Pyrazines are monocyclic aromatic rings with two carbon atoms substituted by nitrogen in the para position. Such compounds occur widely in nature (Müller and Rappert, 2010) although in relatively small amounts (Barlin, 1982). This class of chemicals is a product of the reaction between amino acids, peptides, or proteins and carbonyl compounds described by the non-enzymatic Maillard reaction (Adams and Kimpe, 2009; Yu et al., 2013a, 2017). The highest yields of production are achieved at temperatures between 120 and $150{ }^{\circ} \mathrm{C}$ (Müller and Rappert, 2010) and basic pH values (Jousse et al., 2002; Yu and Zhang, 2010). Pyrazines are also produced in a small scale by biological and fermentation processes (Adams and De Kimpe, 2007; Beck et al., 2003; Larroche et al., 1999), and are major constituents of many aromas and flavours due to their typically low odour threshold value. Pyrazines, particularly alkylpyrazines and alkylmethoxypyrazines, were identified as major scent and flavour contributors (Wagner et al., 1999) in a wide range of food products such as coffee (Blank et al., 1992), wine (Chin et al., 2011), cocoa (Jinap et al., 1998), sweet corn products (Buttery et al., 1994), roasted beef (Cerny and Grosch, 1994), nuts (Alasalvar et al., 2003), orange peel oil (Dharmawan et al., 2009), or tea (Sasaki et al., 2017) among others. Worldwide industrial production of pyrazines is done by chemical synthesis and is limited to a few tons per year. They are used in small amounts by the pharmaceutical industry, while most are used as flavouring ingredients by the food industry (Müller and Rappert, 2010). These are applications where the use of pyrazines is "generally regarded as safe" (Adams et al., 2002).

The physico-chemical properties of hundreds of pyrazines are readily available in the literature and scientific databases. Several molecules within this chemical family exhibit properties of interest for PITT tracer candidates, such as the octanol-water partitioning coefficient $\left(\mathrm{K}_{\mathrm{OW}}\right)$, pKa, or water solubility. Thus, the five pyrazines tested in the present study were selected also by considering the commercial availability and cost of the molecules. The compounds are 2,3-dimethylpyrazine, 2,6-dimethylpyrazine, 2-methoxypyrazine, 2-fluoropyrazine, and 2-chloropyrazine. Some of their relevant physico-chemical properties can be found in Table 1.

Specifically, 2,3-dimethylpyrazine, 2,6-dimethylpyrazine, and 2methoxypyrazine are mostly used as flavouring and/or odorant agents by the food industry (Müller and Rappert, 2010; Rychen et al., 2017),

Table 1

Physicochemical properties of the pyrazines selected as partitioning tracer candidates: melting point, boiling point, octanol/water partition coefficient, and pKa.

\begin{tabular}{lllll}
\hline Compound & Melting point $\left({ }^{\circ} \mathrm{C}\right)$ & Boiling point $\left({ }^{\circ} \mathrm{C}\right)$ & $\mathrm{K}_{\mathrm{OW}}$ & $\mathrm{pKa}^{\mathrm{a}}$ \\
\hline 2,3-Dimethylpyrazine & -12 & 156 & 3,47 & 1,62 \\
2,6-Dimethylpyrazine & 37 & 154 & 3,54 & 1,55 \\
2-Methoxypyrazine & 32 & 169 & 5,37 & 1,51 \\
2-Fluoropyrazine & -6 & 110 & 1,95 & 1,74 \\
2-Chloropyrazine & 20 & 154 & 4,37 & 1,58
\end{tabular}

Data Source: NIST chemistry WebBook.

${ }^{\text {a }}$ Value for the mono-protonated pyrazine. and as a coordination agents for specialty organometallic ligands (Chesnut et al., 2001; Kobayashi et al., 2017; Kong et al., 2006). 2Fluoropyrazine and 2-chloropyrazine are used in the production of special organometallic coordination polymers (Nather et al., 2017; Valverde-Munoz et al., 2016). In addition, several patents describe the use of these two compounds as intermediate reagents in the production of several types of drugs in the pharmaceutical industry.

The presence of these compounds has, to the best of our knowledge, never been described in fluids naturally present in an oil reservoir. Neither have they been mentioned in any type of engineered injected liquids. Hence, their natural occurrence and industrial use suggest it is unlikely that they would be encountered in significant amounts on an oil field. Nevertheless, it is important to stress that the presence of any of the pyrazines under scope of the present study in all injected and produced fluids in an oil-field should be thoroughly assessed prior to their use as tracers in a real tracer test.

\section{Materials and methods}

\subsection{Materials}

2-Fluoropyrazine ( $\geq 97 \%), 2,3$-dimethylpyrazine (99\%), 2,6-dimethylpyrazine ( $\geq 98 \%$ ), 2-methoxypyrazine ( $\geq 98 \%$ ), 2-chloropyrazine ( $\geq 97 \%$ ), kaolinite (natural aluminium silicate $125 \mu \mathrm{m}-250 \mu \mathrm{m}$ with linear formula $\mathrm{Al} 2 \mathrm{O} 3 \cdot 2 \mathrm{SiO} 2 \cdot 2 \mathrm{H} 2 \mathrm{O}$ ) and limestone powder type "BCR-116" (95.7\% CaCO3 + 4.3\% MgCO3) were purchased from Sigma-Aldrich (Sigma-Aldrich Norway AS, 0252 Oslo). Berea sandstone powder [93.13\% silica ( $\mathrm{SiO} 2), 3.86 \%$ alumina (Al2O3), $0.11 \%$ ferric oxide ( $\mathrm{Fe} 2 \mathrm{O} 3$ ), $0.54 \%$ ferrous oxide $(\mathrm{FeO}), 0.25 \%$ magnesium oxide $(\mathrm{MgO})$, and $0.10 \%$ calcium oxide $(\mathrm{CaO})](125 \mu \mathrm{m}-250 \mu \mathrm{m})$ was obtained by crushing and sieving sandstone cores purchased from Berea Sandstone ${ }^{\mathrm{TM}}$ Petroleum Cores (Ohio, USA).

\subsection{Experimental setup and procedures}

Since the current document is the second of three, only a short description with the key experimental elements is presented here. The detailed experimental procedure is described elsewhere: (Silva et al., 2018).

The pyrazines were dissolved in $1 \mathrm{~L}$ of brine with the ion composition shown on Table 2, sonicated and sparged with a flow of $5 \mathrm{~mL} / \mathrm{min}$ of argon during $20 \mathrm{~min} 2 \mathrm{~mL}$ of this pyrazine solution were transferred to 4 sets of glass test tubes containing no rock material, $600 \mathrm{mg}$ of Berea sandstone, $600 \mathrm{mg}$ of kaolinite, and $600 \mathrm{mg}$ of limestone, respectively. The chemical composition of the rock substrates is mostly the same of the rocks that primarily compose sedimentary oil basins. These materials were selected to evaluate the existence of significant interactions (for example, adsorption) with the tracer candidates that could compromise their use in the field. The amount of rock substrate used allowed for a homogenous bulk during the experiments, where no sedimentation was observed during thermal incubation. The glass tubes were then heat-sealed under vacuum. Subsequently, the tubes containing the prepared test samples were incubated in a set of thermal cabinets with rotation mechanisms to provide a gentle agitation. The cabinets were set at temperatures between $25^{\circ} \mathrm{C}$ and $150{ }^{\circ} \mathrm{C}$ with $25^{\circ} \mathrm{C}$ intervals. All tests were conducted in triplicate and three series of tests at initial $\mathrm{pH}$ of 5.5, 7.1 and 8.0 were prepared. The exact concentration of the pyrazines was determined in beginning of the experiment and after thermal incubation during 1, 3, 6, and 12 weeks. Ultra-performance liquid chromatography (UPLC) with UV detection at $222 \mathrm{~nm}$ and $254 \mathrm{~nm}$ was used for quantification of the compounds after filtration of the samples using a $0.45 \mu \mathrm{m}$ PTFE filter. $10 \mu \mathrm{L}$ of the filtered samples were injected on an "Agilent Technologies 1290 Infinity II" UPLC (Agilent Technologies, Santa Clara, California, USA) equipped with a Waters "Acquity UPLC BEH" reversed-phase C18 $1.7 \mu \mathrm{m}$ packed column (Waters Corporation, Milford, Massachusetts, USA). Gradient elution 
Table 2

Composition of the brine used in the experiments.

\begin{tabular}{|c|c|c|c|c|c|c|c|}
\hline $\mathrm{NaCl}(\mathrm{g} / \mathrm{L})$ & $\mathrm{KCl}(\mathrm{g} / \mathrm{L})$ & $\mathrm{CaCl}_{2} \cdot 2 \mathrm{H}_{2} \mathrm{O}(\mathrm{g} / \mathrm{L})$ & $\mathrm{MgCl}_{2} \cdot 6 \mathrm{H}_{2} \mathrm{O}(\mathrm{g} / \mathrm{L})$ & $\mathrm{BaCl}_{2} \cdot 2 \mathrm{H}_{2} \mathrm{O}(\mathrm{g} / \mathrm{L})$ & $\mathrm{SrCl}_{2} \cdot 6 \mathrm{H}_{2} \mathrm{O}(\mathrm{g} / \mathrm{L})$ & $\mathrm{NaHCO}_{3}(\mathrm{~g} / \mathrm{L})^{\mathrm{a}}$ & $\mathrm{Na}_{2} \mathrm{SO}_{4}(\mathrm{~g} / \mathrm{L})$ \\
\hline 36,855 & 0,629 & 3814 & 2550 & 0,088 & 0437 & 0,157 & 0046 \\
\hline
\end{tabular}

${ }^{\text {a }} \mathrm{NaHCO}_{3}$ was used as $\mathrm{pH}$ buffer in varying amounts $(0,157 \mathrm{~g} / \mathrm{L}$ for initial $\mathrm{pH}$ of 7,1; $0.280 \mathrm{~g} / \mathrm{L}$ for initial $\mathrm{pH}$ of 8,0 ; no $\mathrm{NaHCO}$ for initial $\mathrm{pH}$ of 5,5$)$.
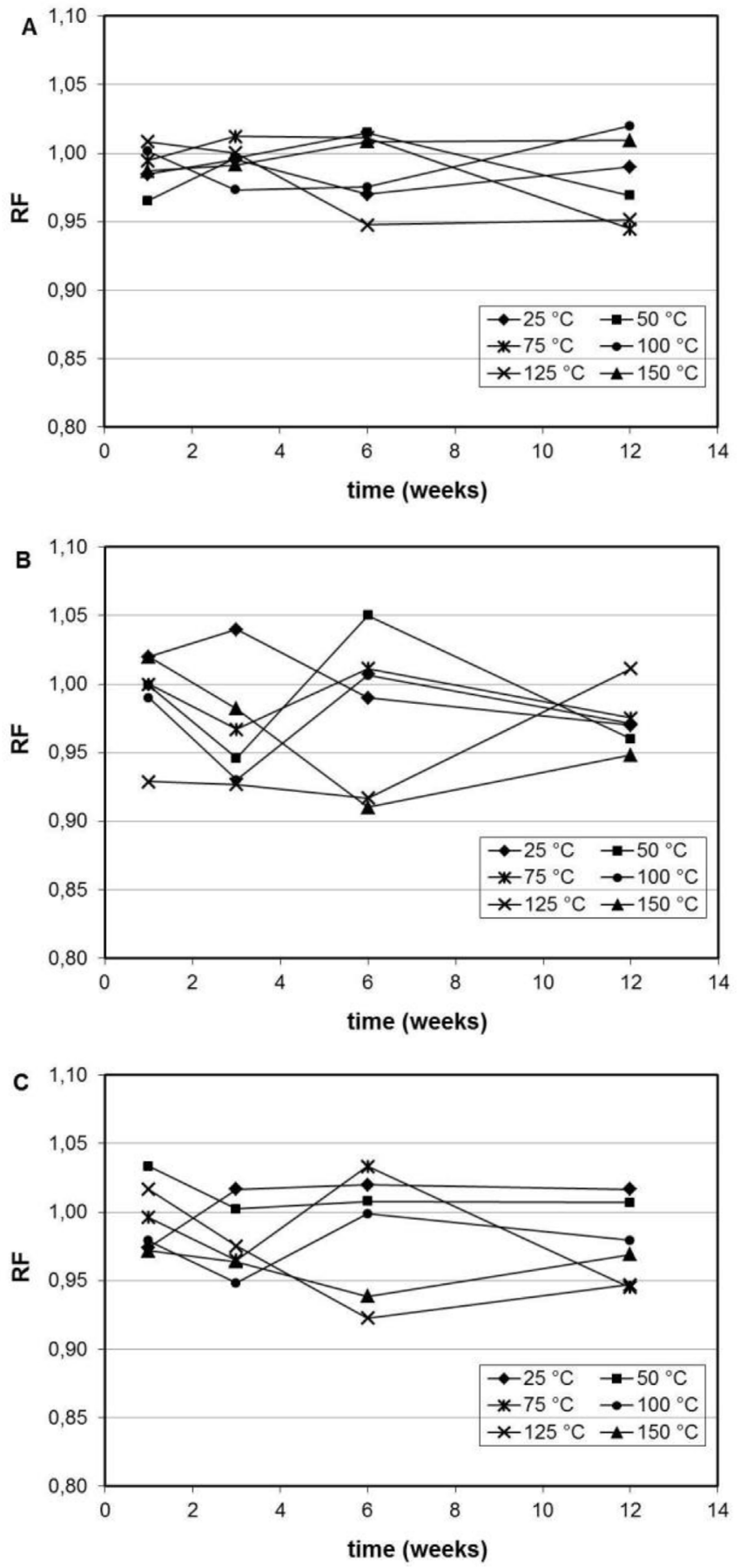

Fig. 1. RF values of 2,3-Dimethylpyrazine as function of time and temperature of incubation. (A) Initial pH 5.5; (B) Initial pH 7.1; (C) Initial pH 8.0 and no rock substrate.

was used for separation of the compounds using a buffer solution of $5 \mathrm{mM} \mathrm{NH}_{4} \mathrm{HCO}_{2}$, methanol, and acetonitrile, at a total constant flow rate of $0.5 \mathrm{~mL} / \mathrm{min}$. The total time of the chromatographic run was $10 \mathrm{~min}$.

\subsection{Degradation kinetics}

To assess the possibility of using the thermally sensitive compounds to retrieve information about temperature conditions in the reservoir, the method of time, based on the integrated rate law, was used for treatment of the data from the thermal stability experiments for such compounds. The mass balance of a component $\mathrm{A}$ in a closed system can be written as (Fogler, 2006):

$K^{\prime} C_{A}^{n}=-\frac{d C_{A}}{d t}$

$\mathrm{C}_{\mathrm{A}}$ is the concentration of compound $\mathrm{A}, \mathrm{K}^{\prime}$ is the reaction's apparent rate constant and $\mathrm{n}$ the order of the reaction. Assuming a first order kinetics and integrating equation (1):

$\ln \frac{C_{A}}{C_{A 0}}=-K^{\prime} t$

$\mathrm{C}_{\mathrm{A} 0}$ corresponds to the initial concentration of compound $\mathrm{A}$. $\mathrm{K}$ is temperature dependent and can be determined by linear regression analysis using the data of the different tested temperatures. The activation energy and pre-exponential factor of $\mathrm{K}$ can be determined from the linearized Arrhenius equation:

$\ln K^{\prime}(T)=\ln A-\frac{E_{a}}{R} \frac{1}{T}$

$A$ is the pre-exponential factor, $E_{a}$ activation energy, $R$ the ideal gas constant, and $\mathrm{T}$ the temperature in Kelvin (K).

\section{Results and discussion}

\subsection{Influence of temperature and time}

The results of the stability experiments are presented as the remaining fraction (RF) of the tracer candidates in the solution as function of time of incubation and temperature. RF is defined as the concentration of the compound in the experimental point divided by its initial concentration. For stable compounds RF should be equal to $1 \pm$ uncertainty in the analytical determination of the concentration of the tracer candidates. The uncertainty is deliberately not depicted in the figures to facilitate their reading, but typical standard deviation values in the determination of $\mathrm{RF}$ from the triplicate experiments range from 0.06 to 0.09 .

The goal of these experiments was to evaluate the ability of the tracer candidates to endure the conditions of temperature and salinity encountered in mature water flooded oil reservoirs. Running a PITT takes several months and obtaining the full production curves can take over one year. It is crucial that the compounds used as tracers do not degrade during the timeframe of the test. Every tracer used must be mass conservative. The experimental conditions intended to be representative of a broad range of possible real field conditions, particularly when temperature is in question. Thus, the conclusions about the thermal stability of the tested pyrazines are not limited to a specific field application, but by the limits of the tested conditions.

No decrease in the RF of 2,3-dimethylpyrazine (2,3-DiM-PRZ) is observable during the incubation period at any tested temperature. Fig. 1 presents the RF values of 2,3-DiM-PRZ as a function of time and temperature of incubation at the three initial $\mathrm{pH}$ values $(5.5 ; 7.1 ; 8.0)$. The variations observed can be explained by the precision of the analytical method used to determine the concentration of the tracer 

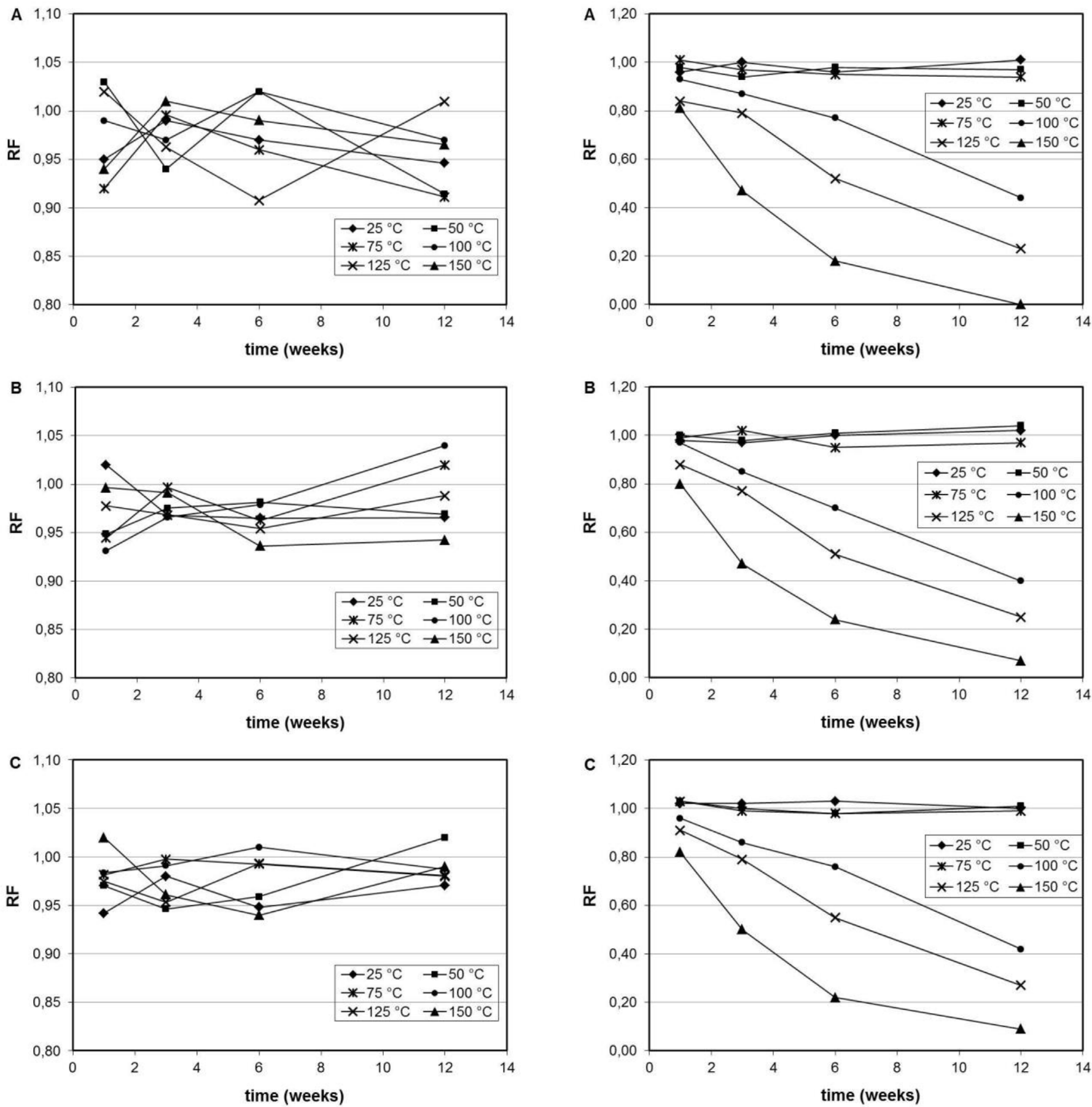

Fig. 2. RF values of 2,6-Dimethylpyrazine as function of time and temperature of incubation. (A) Initial $\mathrm{pH} 5.5$; (B) Initial $\mathrm{pH} 7.1$; (C) Initial $\mathrm{pH} 8.0$ and no rock substrate.

candidates. Results suggest that 2,3-DiM-PRZ possesses the thermal stability required for use as a mass conservative tracer in the oilfield, characteristic crutial for a PITT tracer.

The results depicted in Fig. 2 show that 2,6-dimethylpyrazine (2,6DiM-PRZ) exhibits the thermal stability required for use as partitioning tracer in the oilfield. RF of 2,6-DiM-PRZ remains constant and equal to 1 at all temperatures tested during the total time of experiment. The 12 weeks of thermal incubation (approximately 3 months) are a representative period to evaluate the mass conservation characteristics of an inter-well tracer candidate. The fluctuations of the RF values observed are again explainable by the "normal" uncertainty in the

Fig. 3. RF values of 2-Methoxypyrazine as function of time and temperature of incubation. (A) Initial pH 5.5; (B) Initial $\mathrm{pH} 7.1$; (C) Initial $\mathrm{pH} 8.0$ and no rock substrate.

analytical determination of the concentration of 2,6-DiM-PRZ. It is therefore safe to argue that the use of 2,6-DiM-PRZ is not limited by temperatures up to $150{ }^{\circ} \mathrm{C}$.

The results for 2-Methoxypyrazine (2-MET-PRZ) are presented in Fig. 3. This compound is fully stable during 12 weeks at low temperatures. A decrease in the RF of 2-MET-PRZ is observable in all samples taken at different times of incubation at temperatures $\geq 100^{\circ} \mathrm{C}$. This decrease in the RF value grows both with temperature and length of the experiment. Considering the stability of 2-MET-PRZ at the lowest temperatures, it can be asserted that its degradation is temperature driven and the activation energy barrier is overpassed when the system 
reaches temperatures above $75^{\circ} \mathrm{C}$. An attempt was performed (described in section 2.3.) to develop a first-order kinetic model to describe the decrease in the RF values of 2-MET-PRZ as function of temperature and time. An accurate empirical kinetic model would contribute to validate the hypothesis of a purely temperature-driven degradation of 2-MET-PRZ and open the possibility for the use of this compound to retrieve temperature data of the inter-well region. The use of tracers to retrieve thermal data from reservoirs has been proposed both in geothermal (Schaffer et al., 2016) and oilfield (Silva et al., 2018) applications. A first order kinetic model does not provide an accurate description of the degradation of 2-MET-PRZ and the attempts made to identify the degradation products have proven unsuccessful. The experiments were not performed under sterile conditions. Thus, microbial activity could play a role in the stability of the pyrazines under evaluation. It is widely known that higher temperatures tend to inhibit microbial life and/or growth. Considering the results obtained for 2MET-PRZ (high stability at low temperatures), it is highly unlikely that microbial degradation is being observed. Results generally suggest that, even though not accurately described, temperature driven degradation is affecting the stability of 2-MET-PRZ.

The use of 2-MET-PRZ as mass conservative tracer may still be considered in known low temperature oilfields, but only together with other proven stable tracers which will provide a reference for the total tracer recovery values in a tracer test. Furthermore, the thermally driven degradation of 2-MET-PRZ could even be used to detect thermal fronts in the flooded volumes of the inter-well region, by determining its recovery relatively to one or more stable reference compounds. Calculating the mass recovery of 2-MET-PRZ relatively to a reference mass conservative tracer would provide information about real temperature conditions in the porous medium of the reservoir. For example, if an inter-well tracer test also using 2-MET-PRZ was performed in a low-temperature field and produced a significant decrease of the mass recovery of 2-MET-PRZ, then it would be safe to assume that it swept a volume with an unexpectedly high temperature. Such information could be important for the planning of EOR operations as the methods employed are typically temperature sensitive and rely on conditions which are outputs from reservoir simulations. The validation of the models used in the simulation is based on data obtained in the available wells. Thus, there is no real experimental data about the temperature conditions in the inter-well region. Having a tool for reservoir characterization which will provide, at least qualitative or even semi-quantitative, information about the temperature inside the swept volumes of the reservoir can prove useful for the success of a typically costly EOR campaign.

The presence of covalently bound halogen atoms in tracer candidate compounds ensures that no background effects would compromise their use in the field as it is highly unlikely that the natural formation of halogenated organic molecules would occur under typical oil reservoir conditions. Furthermore, in the specific case of our study, the use of pyrazines tagged with fluoride and chloride facilitates their analysis in real production waters in minor concentrations. These were two additional features leading to the selection of 2-Chloropyrazine (2-Cl-PRZ) and 2-Fluoropyrazine (2-F-PRZ) for testing. The results of the stability experiments are presented in Figs. 4 and 5. Both 2-Cl-PRZ and 2-F-PRZ do not exhibit the stability required for a PITT tracer. The RF value of 2$\mathrm{Cl}-\mathrm{PRZ}$ is decreasing significantly for every tested temperature above $25{ }^{\circ} \mathrm{C}$, and the same is happening above $50{ }^{\circ} \mathrm{C}$ for 2-F-PRZ. The degradation is clearly temperature dependent for both compounds, however a fast and dramatic drop in RF values for 2-F-PRZ is observable at the test temperatures $\geq 100^{\circ} \mathrm{C}$. The experimental data is also inconclusive to assert about a possible concentration dependent kinetics of degradation or about a reaction of order 0 , with a constant degradation rate at a given temperature. An attempt to develop a first order (as described in section 2.3.) kinetic degradation model was performed and a 0-order kinetics was also assessed both for 2,3-Cl-PRZ and 2-F-PRZ. Experimental data isn't accurately described by any of the two models
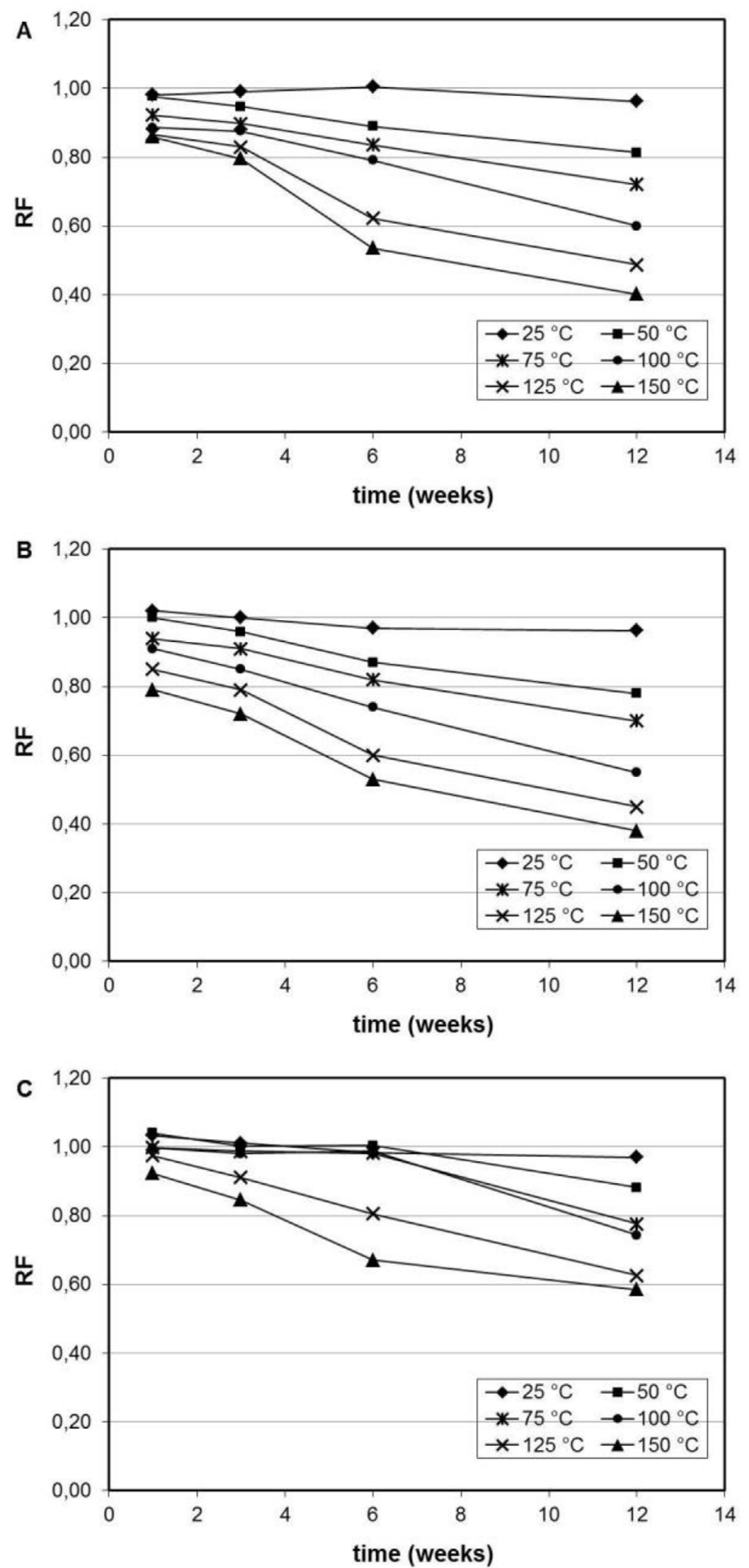

Fig. 4. $\mathrm{RF}$ values of 2-Chloropyrazine as function of time and temperature of incubation. (A) Initial pH 5.5; (B) Initial $\mathrm{pH} 7.1$; (C) Initial $\mathrm{pH} 8.0$ and no rock substrate.

and, without knowledge of the degradation products no further possible use for the 2 compounds was investigated. The decrease of the RF values of 2-Cl-PRZ and 2-F-PRZ observed at relatively low temperatures could be compatible with microbial activity. Temperatures of $25^{\circ} \mathrm{C}$, $50^{\circ} \mathrm{C}$ and $75^{\circ} \mathrm{C}$ allow for the development of many species of microorganisms. Especially when considering that the experimental data is not accurately described by a kinetic model, the possibility of influence of microbial activity on the results should not be discarded. 

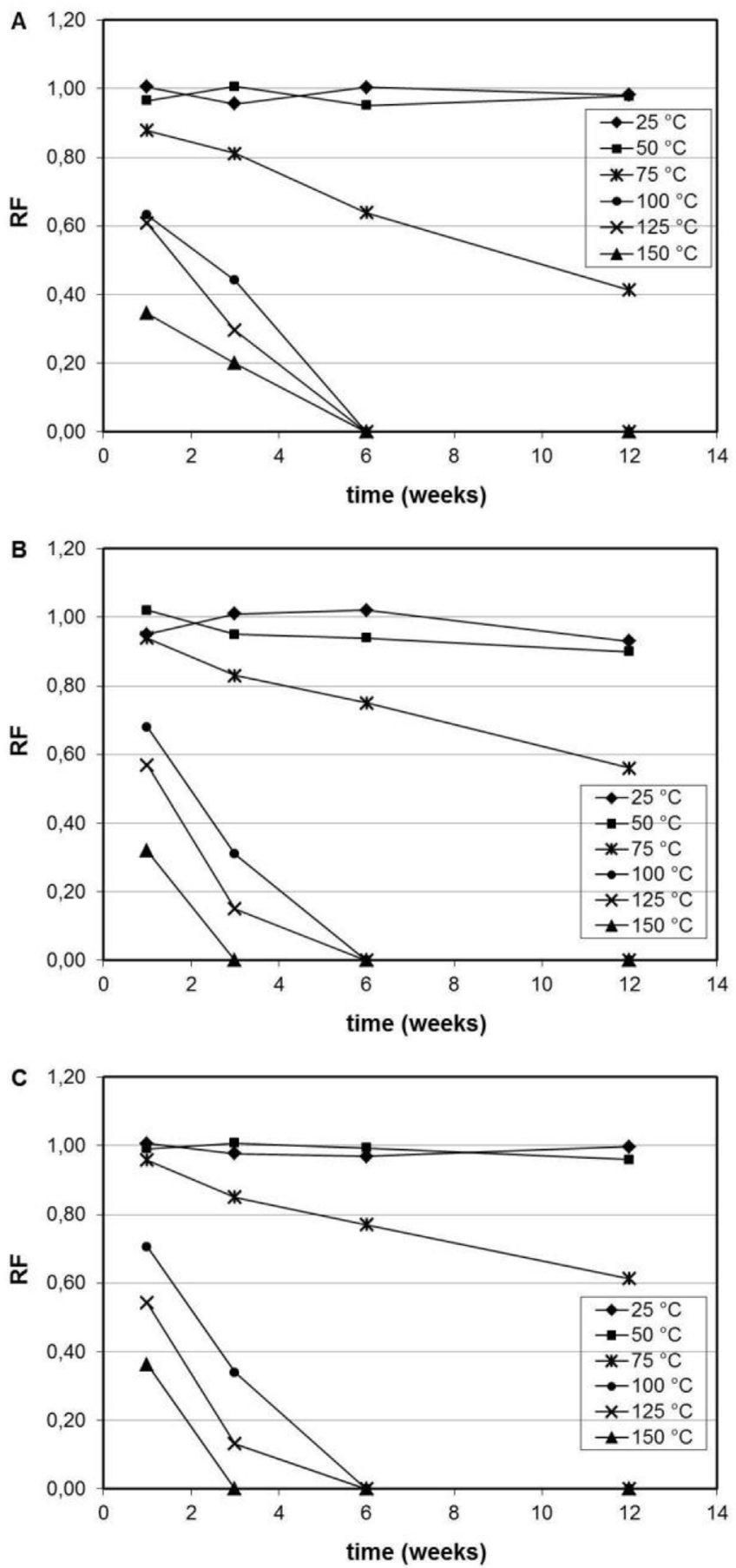

Fig. 5. RF values of 2-Fluoropyrazine as function of time and temperature of incubation. (A) Initial $\mathrm{pH} 5.5$; (B) Initial $\mathrm{pH} 7.1$; (C) Initial $\mathrm{pH} 8.0$ and no rock substrate.

\subsection{Influence of $p H$}

The $\mathrm{pH}$ of the test samples was measured after their incubation period and remained stable even after 12 weeks at all tested temperatures in the experiments performed without any rock substrate. Small variations were observed on the $\mathrm{pH}$ values of the experiments with rock substrate. The maximum variation was a decrease of 0.3 units in the presence of kaolinite as substrate at initial $\mathrm{pH} 7.1$ after 12 weeks of incubation, a rise of $0.2 \mathrm{pH}$ units in the presence of Berea sandstone at initial pH 5.5 after 12 weeks, and an increase of $0.4 \mathrm{pH}$ units in the presence of limestone at initial pH 5.5 after 12 weeks of thermal incubation. The stability of both the alkylpyrazines and 2-methoxypyrazine is not influenced by $\mathrm{pH}$. The RF values of these three compounds are the same for the same time and temperature of incubation regardless of the initial $\mathrm{pH}$ of the experiment (Figs. 1-3). Results suggest that rate of degradation of 2-Cl-PRZ and 2-F-PRZ (Figs. 4 and 5) is influenced by the $\mathrm{pH}$ value of the bulk. In many experimental points, the $\mathrm{RF}$ values appear to be higher as the $\mathrm{pH}$ value increases, however the results (obtained at 3 different $\mathrm{pH}$ values) do not allow for a clear conclusion about this. Most of the data points where the RF values of 2$\mathrm{Cl}-\mathrm{PRZ}$ and 2-F-PRZ are higher at $\mathrm{pH} 8.0$ result from experiments performed at temperatures of $75{ }^{\circ} \mathrm{C}$ and higher, however the differences observed are not enough to preserve the stability of the tracer candidates within a range which would allow considering them for field applications as partitioning tracers under specific $\mathrm{pH}$ conditions.

\subsection{Influence of rock substrate}

Conventional oil basins are primarily constituted by porous sedimentary rocks, either sandstones or carbonate containing other minerals. Substantial amounts of clays are also often present. Clays of different kinds are known to be ion exchangers and can be responsible for irreversible or reversible sorption of tracer compounds. It is therefore critical to assess possible interactions between typical reservoir rock and any mass conservative tracer prior to its field use. Knowledge about such effects ensures the best possible interpretation of the data obtained from the tracer test.

The main criteria for selection of the model rock materials to assess such interactions was its representativeness for the main and real reservoir rock features. By choosing a model rock/mineral one realizes and accepts the fact that they cannot represent every possible geological reservoir formation (Silva et al., 2018). Berea sandstone, limestone and kaolinite were selected as typical oil reservoir rock materials. Fig. 6 illustrates the maximum difference between the RF (t,T) values in the stability experiments with rock substrate and the corresponding RF $(\mathrm{t}, \mathrm{T})$ values without any rock substrate $\pm 2 \sigma$ in the determination of $\mathrm{RF}(\mathrm{t}, \mathrm{T})$.

No difference high enough to exclude 0 from the interval defined by its value $\pm 2 \times \sigma$ was observed for any of the tracer candidates and rock substrates, except for the pair 2-MET-PRZ/kaolinite. This means that even the highest differences encountered in the RF values of all the other 4 pyrazines tested can be justified by the normal experimental/ analytical uncertainty in their determination. Therefore, it is safe to argue that no significant interaction occurs between the tested pyrazines and typical rock materials encountered in sedimentary basins which could compromise their use as oilfield tracers, except for the previously noted exception (the pair 2-MET-PRZ/kaolinite). The full extent of the interaction of kaolinite and 2-MET-PRZ is depicted in Fig. 7. Data points where any of the RF values (with and without kaolinite as substrate) were determined to be inferior to the analytical limit of quantification (LoQ) are not depicted in the figure.

The presence of kaolinite severely reduces the RF of 2-MET-PRZ relatively to the experiments with no rock substrate, unlike the other tested rock materials. Not surprisingly, the extent of the interaction increases with the duration of the experiment. As previously mentioned, kaolinite clays have been described as good adsorbents both for metals and organic compounds (Bhattacharyya and Gupta, 2008; Yu et al., 2013b). Therefore, some adsorption of this tracer candidate compound could occur, however, the results suggest otherwise. Adsorption is typically an exothermal phenomenon. Thus, if adsorption were to be the mechanism behind the interaction, a smaller difference between the RF values would be expected with increasing temperatures for the same incubation periods. The results clearly show that the influence kaolinite has on the RF values of 2-MET-PRZ increases together with temperature. A possible explanation for this observation is that kaolinite is adsorbing the degradation products of the primary compound. It thus follows from Le Chatelier's principle that the equilibrium 

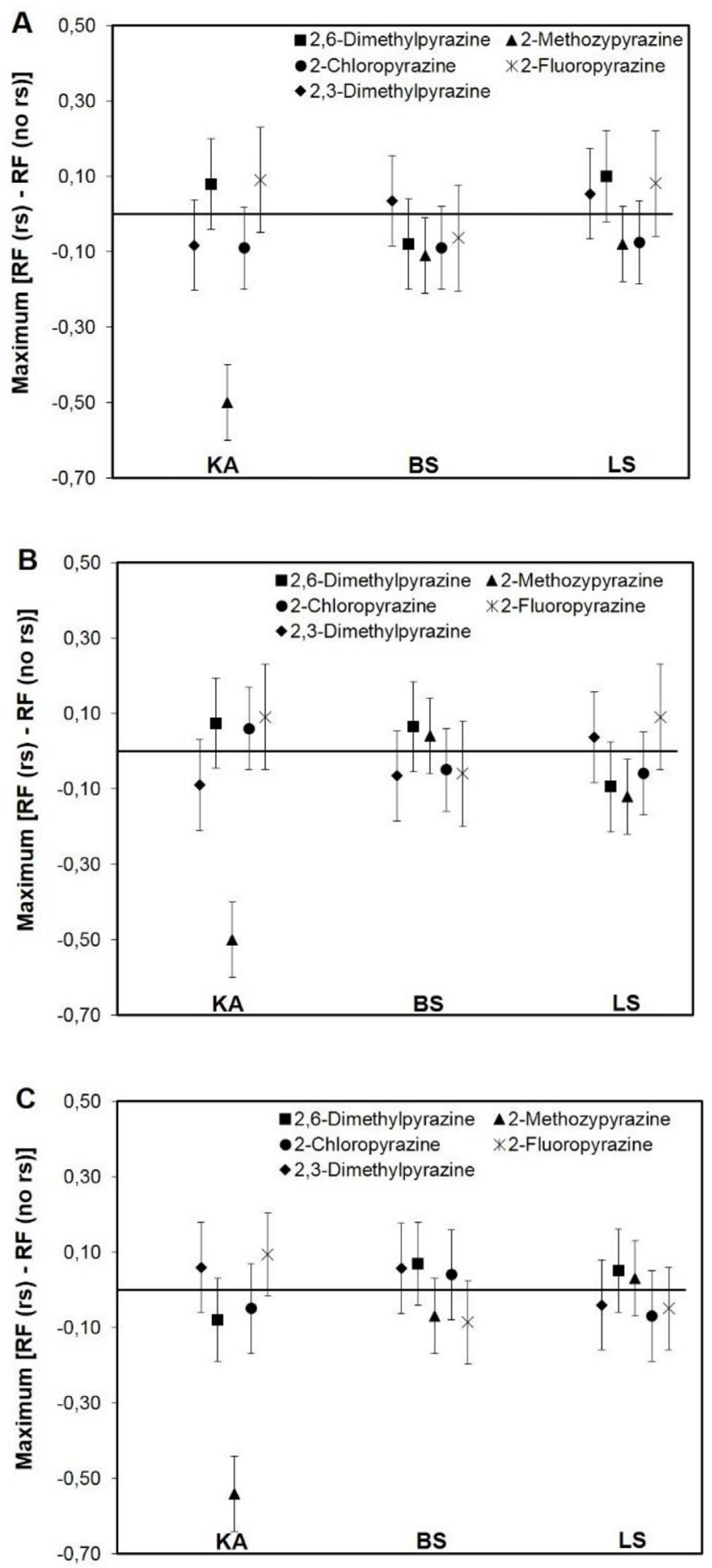

Fig. 6. Maximum differences encountered in RF values between experiments with rock substrate (rs) and experiments without rock substrate $\pm 2 \sigma$ in $R F(t$, T). (KA) kaolinite, (BS) Berea sandstone, (LS) limestone. (A) Initial pH 5.5; (B) Initial pH 7.1; (C) Initial $\mathrm{pH}$ 8.0.

in a possible degradation reaction is shifted towards the right side of the reaction equation:

$2-$ MET - PRZ + Clay $\leftrightarrow($ Clay + A $)(+B)$

Where A and possibly B are adsorbing degradation products. The net result is the enhancement of the reaction rate of the degradation. This effect will continue as long as the absorption capacity of the clay is not saturate, i.e. the adsorption equilibrium between the possible reaction products and the rock substrate is far from being reached. Another

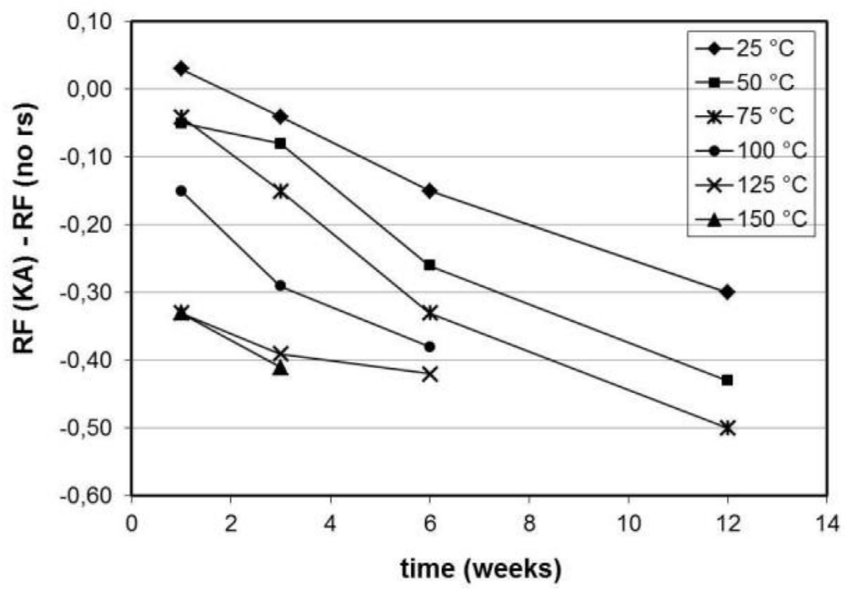

Fig. 7. Differences encountered in the RF values of 2-MET-PRZ between experiments with kaolinite (KA) substrate and experiments without any rock substrate (rs).

possible explanation is that kaolinite is acting as catalyst for the reaction of degradation of 2-MET-PRZ. Even at temperature values $\left(25^{\circ} \mathrm{C}\right.$, $50^{\circ} \mathrm{C}$, and $75^{\circ} \mathrm{C}$ ) where no decrease on the $R F$ value is observed in the experiments without rock substrate, a substantial reduction of RF values is observed in presence of kaolinite. This is in accordance with the catalysis hypothesis, as the main effect of catalysts is to reduce the activation energy of the reaction they are catalyzing. The use of kaolinite as catalyst has been reported in the literature (Li et al., 2019; Vorlicek and Helz, 2002) and it has been the target of studies of enhancement of catalytical properties (Belver et al., 2002). These assumptions are however not validated as so far, we have been unable to identify the products of the degradation of 2-Methoxypyrazine.

\section{Conclusions}

As part of the qualification process of new oil/water partitioning tracers for the determination of residual oil saturation in the inter-well region of oil reservoirs, a batch stability assessment was performed on two alkylpyrazines, one Methoxypyrazine, and two halogenated pyrazines. The compounds were tested in a brine solution to account for the effect of high ion strength, and the experiments lasted up to 12 weeks. The influence of temperature in the range of $25^{\circ} \mathrm{C}-150{ }^{\circ} \mathrm{C}$, and $\mathrm{pH}$ in the range of 5.5-8.0 were evaluated. Kaolinite, Berea sandstone, and limestone were used as model reservoir rock materials to study and characterize possible interactions with the tracer candidate compounds.

The stability of 2,3-dimethylpirazine, 2,6-dimethylpyrazine and 2methoxypyrazine is not influenced by the $\mathrm{pH}$ within the studied range, while a small effect is observed on 2-chloropyrazine and 2-fluroropyrazine. An argument can be made that a decrease of the degradation rate is observed for 2-chloropyrazine and 2-fluroropyrazine with increasing $\mathrm{pH}$ values, however results are not clear about it.

2,3-Dimethylpirazine and 2,6-dimethylpyrazine appear to be fully stable at all tested conditions. No degradation is observable during the 12 weeks of testing and under temperatures up to $150{ }^{\circ} \mathrm{C}$, in the absence or presence of rock substrates. Furthermore, no type of interaction between these two chemicals and the rock materials was evidenced by the results.

2-Methoxypyrazine is highly stable up to the test temperature of $75^{\circ} \mathrm{C}$ in the absence of any rock materials and in the presence of sandstone and limestone. A relatively fast degradation of this compound is observed at temperatures above $75^{\circ} \mathrm{C}$ with a clearly temperature dependent rate which is not well described by an 0 -order or 1order kinetic model. The presence of kaolinite has a dramatic influence on the stability of this compound, and a significant decrease in its remaining fraction values is observed after a short period of time even at 
low temperature experiments. At higher temperatures, 2-methoxypyrazine disappears from the solution at rates significantly higher than in the experiments without any rock substrate or in the presence of the other 2 tested when kaolinite is present, but the nature of the mechanism behind this effect is not clear.

2-Chloropyrazine exhibits limited stability. Absence of significant degradation was observed only at $25^{\circ} \mathrm{C}$. The degradation rate of this compound increases with increasing temperatures and is not influenced by the presence of any of the 3 rock substrates tested. This compound is unsuited for use as partitioning tracer for stability reasons.

2-Fluoropyrazine is also unsuited for use as partitioning tracer in an oilfield with temperatures higher than $50-60{ }^{\circ} \mathrm{C}$. It is highly stable at $25^{\circ} \mathrm{C}$ and $50{ }^{\circ} \mathrm{C}$ up to 12 weeks. Results show a fast degradation of this compound at temperatures equal and higher than $75^{\circ} \mathrm{C}$. The rate of degradation increases with increasing temperatures and is independent of the presence of any of the tested rock substrates.

\section{Acknowledgements}

The authors acknowledge the Research Council of Norway and the industry partners, ConocoPhillips Skandinavia AS, Aker BP ASA, Eni Norge AS, Maersk Oil, a company by Total, Statoil Petroleum AS, Neptune Energy Norge AS, Lundin Norway AS, Halliburton AS, Schlumberger Norge AS, Wintershall Norge AS, and DEA Norge AS, of The National IOR Centre of Norway for economical support.

\section{Appendix A. Supplementary data}

Supplementary data to this article can be found online at https:// doi.org/10.1016/j.petrol.2019.106269.

\section{References}

Adams, A., De Kimpe, N., 2007. Formation of pyrazines and 2-acetyl-1-pyrroline by Bacillus cereus. Food Chem. 101 (3), 1230-1238.

Adams, A., Kimpe, N.D., 2009. Formation of pyrazines from ascorbic acid and amino acids under dry-roasting conditions. Food Chem. 115 (4), 1417-1423.

Adams, T.B., et al., 2002. The FEMA GRAS assessment of pyrazine derivatives used as flavor ingredients. Food Chem. Toxicol. 40 (4), 429-451.

Al-Murayri, M.T., Al-Qenae, A., AlRukaibi, D., Chatterjee, M., Hewitt, P., 2017. Design of a partitioning interwell tracer test for a chemical EOR pilot targeting the Sabriyah Mauddud carbonate reservoir in Kuwait (SPE-187549-MS) In: SPE Kuwait Oil \& Gas Show and Conference. Society of Petroleum Engineers, Kuwait City, Kuwait.

Alasalvar, C., Shahidi, F., Cadwallader, K.R., 2003. Comparison of natural and roasted Turkish tombul hazelnut (Corylus avellana L.) volatiles and flavor by DHA/GC/MS and descriptive sensory analysis. J. Agric. Food Chem. 51 (17), 5067-5072.

Barlin, G.B., 1982. The Chemistry of Heterocyclic Compounds - the Pyrazines, vol. 41 John Wiley \& Sons, New York.

Beck, H.C., Hansen, A.M., Lauritsen, F.R., 2003. Novel pyrazine metabolites found in polymyxin biosynthesis by Paenibacillus polymyxa. FEMS Microbiol. Lett. 220 (1), 67-73.

Belver, C., Bañares Muñoz, M.A., Vicente, M.A., 2002. Chemical activation of a kaolinite under acid and alkaline conditions. Chem. Mater. 14 (5), 2033-2043.

Bhattacharyya, K.G., Gupta, S.S., 2008. Adsorption of a few heavy metals on natural and modified kaolinite and montmorillonite: a review. Adv. Colloid Interface Sci. 140 (2), 114-131.

Blank, I., Sen, A., Grosch, W., 1992. Potent odorants of the roasted powder and brew of Arabica coffee. Z. Lebensm. Unters. Forsch. 195 (3), 239-245.

Buttery, R.G., Stern, D.J., Ling, L.C., 1994. Studies on flavor volatiles of some sweet corn products. J. Agric. Food Chem. 42 (3), 791-795.

Cerny, C., Grosch, W., 1994. Precursors of ethyldimethylpyrazine isomers and 2,3-diethyl-5-methylpyrazine formed in roasted beef. Z. Lebensm. Unters. Forsch. 198 (3), 210-214.

Chesnut, D.J., Plewak, D., Zubieta, J., 2001. Solid state coordination chemistry of the copper(I)-cyano-organodiimine system. Two- and three-dimensional copper cyanide phases incorporating linear dipodal ligands. J. Chem. Soc. Dalton Trans. 18, 2567-2580.

Chin, S.-T., Eyres, G.T., Marriott, P.J., 2011. Identification of potent odourants in wine and brewed coffee using gas chromatography-olfactometry and comprehensive twodimensional gas chromatography. J. Chromatogr. A 1218 (42), 7487-7498.
Cooke, C.E.J., 1971. "Method of Determining Fluid Saturations in Reservoirs" (US Patent 3,590,923). Esso Production Research Company, USA.

Dean, R.M., Walker, D.L., Dwarakanath, V., Malik, T., Spilker, K., 2016. (SPE-179655-MS) "use of partitioning tracers to estimate oil saturation distribution in heterogeneous reservoirs. In: SPE Improved Oil Recovery Conference. Society of Petroleum Engineers, Tulsa, Oklahoma, USA.

Dharmawan, J., Kasapis, S., Sriramula, P., Lear, M.J., Curran, P., 2009. Evaluation of aroma-active compounds in pontianak orange peel oil (citrus nobilis lour. Var. Microcarpa hassk.) by gas chromatography-olfactometry, aroma reconstitution, and omission test. J. Agric. Food Chem. 57 (1), 239-244.

Fogler, H.S., 2006. Elements of Chemical Reaction Engineering. Prentice Hall PTR, New Jersey.

Huseby, O., Hartvig, S.K., Jevanord, K., Dugstad, Ø., 2015. Assessing EOR potential from partitioning tracer data (SPE-172808-MS). In: SPE Middle East Oil \& Gas Show and Conference. Society of Petroleum Engineers, Manama, Bahrain.

Jin, M., et al., 1995. Partitioning tracer test for detection, estimation, and remediation performance assessment of subsurface nonaqueous phase liquids. Water Resour. Res. 31 (5), 1201-1211.

Jinap, S., Rosli, W.I.W., Russly, A.R., Nordin, L.M., 1998. Effect of roasting time and temperature on volatile component profiles during nib roasting of cocoa beans (Theobroma cacao). J. Sci. Food Agric. 77 (4), 441-448.

Jousse, F., Jongen, T., Agterof, W., Russell, S., Braat, P., 2002. Simplified kinetic scheme of flavor formation by the Maillard reaction. J. Food Sci. 67 (7), 2534-2542.

Kobayashi, A., Arata, R., Ogawa, T., Yoshida, M., Kato, M., 2017. Effect of water coordination on luminescent properties of pyrazine-bridged dinuclear $\mathrm{Cu}(\mathrm{I})$ complexes. Inorg. Chem. 56 (8), 4280-4288.

Kong, X.J., et al., 2006. Construction of polyoxometalates-based coordination polymers through direct incorporation between polyoxometalates and the voids in a $2 \mathrm{D}$ network. Inorg. Chem. 45 (26), 10702-10711.

Larroche, C., Besson, I., Gros, J.-B., 1999. High pyrazine production by Bacillus subtilis in solid substrate fermentation on ground soybeans. Process Biochem. 34 (6), 667-674.

Leo, A., Hansch, C., Elkins, D., 1971. Partition coefficients and their uses. Chem. Rev. 71 (6), 525-616.

Li, C., et al., 2019. Highly efficient activation of peroxymonosulfate by natural negativelycharged kaolinite with abundant hydroxyl groups for the degradation of atrazine. Appl. Catal. B Environ. 247, 10-23.

McDonald, K., Carroll, K.C., Brusseau, M.L., 2016. Comparison of fluid-fluid interfacial areas measured with X-ray microtomography and interfacial partitioning tracer tests for the same samples. Water Resour. Res. 52 (7), 5393-5399.

Müller, R., Rappert, S., 2010. Pyrazines: occurrence, formation and biodegradation. Appl. Microbiol. Biotechnol. 85 (5), 1315-1320.

Nather, C., et al., 2017. CdX2 coordination polymers with 2-chloropyrazine and 2-methylpyrazine: similar ligands - similar structures different reactivity. Eur. J. Inorg. Chem. (9), 1245-1255.

Rychen, G., et al., 2017. Safety and efficacy of pyrazine derivatives including saturated ones belonging to chemical group 24 when used as flavourings for all animal species. Efsa J. 15 (2), 18.

Sanni, M., et al., 2018. Pushing the envelope of residual oil measurement: a field case study of a new class of inter-well chemical tracers. J. Pet. Sci. Eng. 163, 538-545.

Sasaki, T., et al., 2017. Characterisation of odorants in roasted stem tea using gas chromatography-mass spectrometry and gas chromatography-olfactometry analysis. Food Chem. 220, 177-183.

Schaffer, M., Idzik, K.R., Wilke, M., Licha, T., 2016. Amides as thermo-sensitive tracers for investigating the thermal state of geothermal reservoirs. Geothermics 64, 180-186.

Silva, M., Stray, H., Bjørnstad, T., 2017. New potential tracer compounds for inter-well SOR determination - stability at reservoir conditions. In: IOR 2017 - 19th European Symposium on Improved Oil Recovery EAGE (European Association of Geoscientists \& Engineers), Stavanger, Norway.

Silva, M., Stray, H., Bjørnstad, T., 2018. Stability assessment of PITT tracer candidate compounds: the case of benzyl alcohols. J. Pet. Sci. Eng. 167, 517-523.

Valverde-Munoz, F.J., et al., 2016. Strong cooperative spin crossover in 2D and 3D Fe-IIM-I,M-II hofmann-like coordination polymers based on 2-fluoropyrazine. Inorg. Chem. 55 (20), 10654-10665.

Vorlicek, T.P., Helz, G.R., 2002. Catalysis by mineral surfaces: implications for Mo geochemistry in anoxic environments. Geochem. Cosmochim. Acta 66 (21), 3679-3692.

Wagner, R., Czerny, M., Bielohradsky, J., Grosch, W., 1999. Structure-odour-activity relationships of alkylpyrazines. Z. Lebensm. -Forsch. A 208 (5), 308-316.

Wang, F., Annable, M.D., Jawitz, J.W., 2013. Field-scale prediction of enhanced DNAPL dissolution based on partitioning tracers. J. Contam. Hydrol. 152, 147-158.

Yu, A.-N., Tan, Z.-W., Wang, F.-S., 2013a. Mechanistic studies on the formation of pyrazines by Maillard reaction between 1-ascorbic acid and l-glutamic acid. LWT - Food Sci. Technol. (Lebensmittel-Wissenschaft -Technol.) 50 (1), 64-71.

Yu, A.-N., Zhang, A.-D., 2010. The effect of $\mathrm{pH}$ on the formation of aroma compounds produced by heating a model system containing l-ascorbic acid with l-threonine/1serine. Food Chem. 119 (1), 214-219.

Yu, A.-N., Zhou, Y.-Y., Yang, Y.-N., 2017. Kinetics of browning and correlations between browning degree and pyrazine compounds in 1-ascorbic acid/acidic amino acid model systems. Food Chem. 221, 1678-1684.

Yu, W.H., et al., 2013b. Adsorption of proteins and nucleic acids on clay minerals and their interactions: a review. Appl. Clay Sci. 80-81, 443-452. 



\section{Paper V}

Thermal stability and interactions with sedimentary rocks under typical reservoir conditions of selected pyridines investigated as phase partitioning tracers.

Mário Silva, Helge Stray, Mahmoud Ould Metidji, Tor Bjørnstad (2021)

Upstream Oil and Gas Technology, (6) 100033. DOI:

10.1016/j.upstre.2021.100033. 


\title{
Thermal stability and interactions with sedimentary rocks under typical reservoir conditions of selected pyridines investigated as phase partitioning
} tracers

\author{
Mario Silva ${ }^{\mathrm{a}, \mathrm{b}, \mathrm{c}, *}$, Helge Stray ${ }^{\mathrm{c}}$, Mahmoud Ould Metidji ${ }^{\mathrm{a}, \mathrm{c}}$, Tor Bjørnstad ${ }^{\mathrm{a}, \mathrm{c}}$ \\ a The National IOR Centre of Norway, University of Stavanger, 4036 Stavanger, Norway \\ ${ }^{\mathrm{b}}$ Department of Energy Resources, University of Stavanger, 4036 Stavanger, Norway \\ ${ }^{\mathrm{c}}$ Institute for Energy Technology (IFE), Department of Tracer Technology, Instituttveien 18, 2007 Kjeller, Norway
}

\section{A R T I C L E I N F O}

\section{Keywords:}

Partitioning tracers

Stability

Reservoir rocks

New compounds

Pyridines

\begin{abstract}
A B S T R A C T
Pyridine, 2-hydroxy-6-methylpyridine, 3-hydroxypyridine, and 4-methoxypyridine are evaluated as potential phase-partitioning oil field tracers. Their stability is tested in a brine for 12 weeks at temperatures between $25^{\circ} \mathrm{C}-$ $150{ }^{\circ} \mathrm{C}$, and at initial $\mathrm{pH}$ values of 5,$5 ; 7,1 ; 8,0$. Interactions with kaolinite clay, Berea sandstone and limestone are also evaluated. The main results are as follows: pyridine is stable up to 12 weeks at $150{ }^{\circ} \mathrm{C}$, and not influenced by the rock substrates or $\mathrm{pH}$. 2-hydroxy-6-methylpyridine becomes unstable at $\mathrm{T} \geq 50{ }^{\circ} \mathrm{C}$, is not affected by the rock substrates, and exihibts slower degradation kinetics at higher $\mathrm{pH}$ values. 3-hydroxypyridine is unstable at $\mathrm{T} \geq 75{ }^{\circ} \mathrm{C}$, sensitive to the presence of kaolinite in a combined effect with $\mathrm{pH}$, and exihibts slower degradation kinetics at higher $\mathrm{pH}$. 4-methoxypyridine degrades at $\mathrm{T} \geq 75{ }^{\circ} \mathrm{C}$, is characterised by a strong interaction with kaolinite, and is insensitive to $\mathrm{pH}$.

The degradation of 4-methoxypyridine in the absence of kaolinite clay follows pseudo first-order kinetics. This compound could indicate the temperature in the swept volumes, and in conjunction with a fully conservative tracer indicate the presence of clays. Pyridine exhibits the required stability and lack of interaction with rock materials to be used as PITT tracer in oil reservoirs. However, it is present in oils and its concentration levels in production waters should be evaluated prior to its use.
\end{abstract}

\section{Introduction}

Knowledge of the residual oil saturation $\left(\mathrm{S}_{\mathrm{OR}}\right)$ in the swept volumes of oil reservoirs can be used both to improve reservoir management, and in the design and evaluation of EOR/IOR projects [1,2]. As the number of mature fields increases [3], such projects become more important to ensure the production of hydrocarbons to satisfy global demand. A partitioning inter-well tracer test (PITT) is the only dynamic tool to measure $\mathrm{S}_{\mathrm{OR}}$ in the inter-well region of waterflooded reservoirs. A PITT is based on the use of passive and oil/water partitioning tracers [4]. It was introduced to the oil industry in 1971 by Cooke [5], however never routinely used. The poor knowledge about the behaviour and geochemical interactions of the compounds used as PITT tracers lead to many unsuccessful tests in the past $[4,6]$. In recent years, this type of tracer test has received increasing attention due to the importance of the information it provides $[7,8]$. Additionally, a small number of compounds has been developed for use as PITT tracers [6,9]; additional PITT tracers would be useful. The study of tracer compounds prior to their use on the field is crucial, not only to ensure the accuracy of the tests, but also to evaluate the possibility of using tracers to retrieve other type of relevant information [10-12].

In this document we present the stability assessment performed on 4 pyridines that are PITT tracer candidates. The influence of temperature, $\mathrm{pH}$, time, salinity, and different rock materials, on the stability and behaviour of pyridine, 4-methoxypyridine, 3-hydroxypyridine, and 2-Hydroxy-6-methylpyridine was investigated.

\subsection{Pyridines}

Pyridine has the chemical formula $\mathrm{C}_{5} \mathrm{H}_{5} \mathrm{~N}$ and a structure similar to benzene. It is a weakly basic, transparent "yellowish", flammable, and water-soluble heterocyclic aromatic compound, with a distinct highly unpleasant smell. Such properties are also observed in many of the different substituted pyridines. Many compounds within this family of chemicals show interesting properties for use as PITT tracers. Relevant properties of the compounds tested here can be found in Table 1.

\footnotetext{
* Corresponding author at: Institute for Energy Technology (IFE), Department of Tracer Technology, Instituttveien 18, 2007 Kjeller, Norway.

E-mail addresses: mario.silva@ife.no, mariohsilva@sapo.pt (M. Silva).
} 
Table 1

Melting point, boiling point, $\mathrm{pKa}$, and octanol/water partitioning coefficient of the pyridines selected as partitioning tracer candidates.

\begin{tabular}{lllll}
\hline Compound & Melting point $\left({ }^{\circ} \mathrm{C}\right)$ & Boiling point $\left({ }^{\circ} \mathrm{C}\right)$ & $\mathrm{K}_{\mathrm{OW}}$ & $\mathrm{pKa}$ \\
\hline Pyridine & -42 & 115 & 4,47 & $5,25^{*}$ \\
4-Methoxypyridine & 4 & 191 & 5,22 & $6,58^{*}$ \\
3-Hydroxypyridine & 126 & 329 & 4,36 & $4,79^{*} / 8,75$ \\
2-Hydroxy-6-methylpyridine & 159 & 275 & 2,18 & $4,94^{*} / 9,27$ \\
Data Source: NIST chemistry WebBook & & & \\
\hline
\end{tabular}

*Value for the mono-protonated pyridine.

Pyridine and polycyclic pyridine derivatives have been reported in crude oils and are commonly classified as part of their alkaline fraction [13-15]. They are responsible for $20 \%-26 \%$ of the total nitrogen content in all crude oils $[13,14]$ which is usually $\leq 1 \%$ (wt), and $<0.25 \%$ (wt) in about $90 \%$ of them [13]. The other pyridine derivatives investigated under the scope of this work have never, to the best of our knowledge, been reported as constituents of any type of oil reservoir fluid. The chemical industry uses pyridine as raw-material and intermediate on a large scale. It is the precursor to some pesticides, such as paraquat and diquat [16], industrially used as solvent, in the production of dyes, explosives, pharmaceuticals, etc. $[17,18]$, and is used as a ligand (or base unit for ligands) in coordination chemistry [19]. 4-Methoxypyridine is mostly used as a ligand in coordination chemistry $[20,21]$, and in the synthesis of pharmaceutical compounds [22]. 3-Hydroxypyridine has been considered as a precursor for the production of nitrogen-rich polymers for $\mathrm{CO}_{2}$ adsorption [23], used as additive to improve optical properties of hydrophilic ophthalmic lenses [24], and also as precursor for pharmaceutical active principles [25]. 2-Hydroxy-6-methylpyridine has no significant industrial use and we only found it reported as a ligand in the preparation of metal-lanthanide complexes or special metal complexes $[26,27]$. The widespread use of pyridines means that most of them are available at lower cost than the compounds presently used as PITT tracers.

Pyridines are probably one of the least obvious choices for use as PITT tracer candidates because of two main issues: i) pyridine itself has been identified as a minor constituent of crude oils; ii) pyridine and its derivatives are weak bases, and therefore $\mathrm{pH}$ sensitive compounds when in aqueous solution. However, the PITT is primarily designed for mature oilfields where waterflooding has been performed for long periods of time (typically years). In such fields, it can be expected that a chemical equilibrium (or quasi-equilibrium) is established between the hydrocarbons and the circulating aqueous phase, which will keep the dissolution of pyridines in the water at a low concentration. Such conditions will not affect the use of pyridines as PITT tracers, as the background "noise" will not compromise detection of the pyridine injected as tracer. A recent study found no evidence of the presence of pyridine in production waters from eight oilfields on the Norwegian continental shelf [28]. The use of deuterated forms of pyridine, could also be used to distinguish the PITT tracer from natural pyridine. The $\mathrm{pH}$ sensitivity of this family of compounds could indicate $\mathrm{pH}$ conditions inside the porous medium. The partition coefficient $(\mathrm{K})$ of pyridines varies as function of $\mathrm{pH}$.

\section{Materials and methods}

\subsection{Materials}

Pyridine ( $\geq 99 \%$ ), 4-methoxypyridine ( $\geq 99 \%$ ), 3-hydroxypyridine ( $\geq 98 \%)$ 2-hydroxy-6-methylpyridine $(\geq 97 \%)$, kaolinite (natural aluminium silicate $125 \mu \mathrm{m}-250 \mu \mathrm{m}$ with linear formula $\mathrm{Al} 2 \mathrm{O} 3 \cdot 2 \mathrm{SiO} 2 \cdot 2 \mathrm{H} 2 \mathrm{O})$ and limestone powder type "BCR-116" $(95.7 \%$ $\mathrm{CaCO} 3+4.3 \% \mathrm{MgCO} 3$ ) were purchased from Sigma-Aldrich (SigmaAldrich Norway AS, 0252 Oslo). Berea sandstone powder [93.13\% silica $\left(\mathrm{SiO}_{2}\right), 3.86 \%$ alumina $\left(\mathrm{Al}_{2} \mathrm{O}_{3}\right), 0.11 \%$ ferric oxide $\left(\mathrm{Fe}_{2} \mathrm{O}_{3}\right)$, $0.54 \%$ ferrous oxide $(\mathrm{FeO}), 0.25 \%$ magnesium oxide $(\mathrm{MgO})$, and $0.10 \%$ calcium oxide (CaO)] $(125 \mu \mathrm{m}-250 \mu \mathrm{m})$ was obtained by crushing and sieving sandstone cores purchased from Berea Sandstone ${ }^{\mathrm{TM}}$ Petroleum Cores (Ohio, USA).

\subsection{Experimental procedure}

$1 \mathrm{~L}$ of $10 \mathrm{mg} \mathrm{L}^{-1}$ solution of the selected pyridines was prepared in a synthetic brine. The brine was constituted of $36.855 \mathrm{~g} \mathrm{~L}^{-1}$ of $\mathrm{NaCl}, 0.629 \mathrm{~g} \mathrm{~L}^{-1}$ of $\mathrm{KCl}, 3.814 \mathrm{~g} \mathrm{~L}^{-1}$ of $\mathrm{CaCl}_{2} .2 \mathrm{H}_{2} \mathrm{O}, 2.550 \mathrm{~g} \mathrm{~L}^{-1}$ of $\mathrm{MgCl}_{2} \cdot 6 \mathrm{H}_{2} \mathrm{O}, 0.088 \mathrm{~g} \mathrm{~L}^{-1}$ of $\mathrm{BaCl}_{2} .2 \mathrm{H} 2 \mathrm{O}, 0.437 \mathrm{~g} \mathrm{~L}^{-1}$ of $\mathrm{SrCl}_{2} .6 \mathrm{H} 2 \mathrm{O}$, and $0.046 \mathrm{~g} \mathrm{~L}^{-1}$ of $\mathrm{Na}_{2} \mathrm{SO}_{4}$. The $\mathrm{pH}$ of this solution was 5.5. Two other solutions to test the compounds at $\mathrm{pH} 7.1$ and 8.0 were prepared using of $\mathrm{NaHCO}_{3}$ to adjust the $\mathrm{pH}$ values.

Oxygen was removed from the solutions through sonication and sparging with $5 \mathrm{~mL} / \mathrm{min}$ of argon for $20 \mathrm{~min}$. The solutions were kept under constant sparging of argon during the whole time of sample preparation. 4 sets of durex glass test tubes were prepared: 1 set containing no rock substrate, 1 set containing $600 \mathrm{mg}$ of kaolinite, 1 set containing $600 \mathrm{mg}$ of Berea sandstone, and 1 set containing $600 \mathrm{mg}$ of limestone. $2 \mathrm{~mL}$ of the solution of the tracer candidates was transferred to the test tubes. Vacuum was applied to the headspace of the test tubes during their heat-sealing. The experiments were performed in triplicate. The test tubes were then incubated during 12 weeks at temperatures up to $150{ }^{\circ} \mathrm{C}$ in thermal cabinets. The thermal cabinets had a rotation mechanism which ensured smooth agitation and homogeneity of the bulk during the experimental period. The concentration of the tested pyridines was monitored after $1,3,6$, and 12 weeks of incubation. The quantification of the compounds was done by UPLC-UV with detection at 222 and $254 \mathrm{~nm}$, by injecting $10 \mu \mathrm{L}$ of the test samples filtered through a $0.45 \mu \mathrm{m}$ PTFE filter into the chromatographic system. A "Agilent Technologies 1290 Infinity II" UPLC (Agilent Technologies, Santa Clara, California, USA) equipped with a Waters "Acquity UPLC BEH" reversedphase C18 $1.7 \mu \mathrm{m}$ packed column (Waters Corporation, Milford, Massachusetts, USA) was used. Elution of all pyridines was achieved with a gradient with three eluents in the mobile phase. These were a buffer solution of $5 \mathrm{mM} \mathrm{NH} 4 \mathrm{HCO} 2$, methanol, and acetonitrile, at a total constant flow rate of $0.5 \mathrm{~mL} / \mathrm{min}$. The total time of the chromatographic run was $10 \mathrm{~min}$.

\section{Results and discussion}

Results are presented in the form of remaining fraction (RF) as function of time $(\mathrm{t})$ and temperature $(\mathrm{T})$. RF of the tested pyridines is defined as $C(t, T) / C\left(t_{0}, T_{0}\right)$. Analytical uncertainty is deliberately not depicted to facilitate the reading of the figures. Typical standard deviation values $(\sigma)$ in the determination of the concentration in the triplicate replicas range from 0,04 to 0,08 .

\subsection{Pyridine}

Fig. 1 depicts the RF values of pyridine as function of time and temperature at the different initial $\mathrm{pH}$ values.

No clear trend is observable for the RF values of pyridine (Fig. 1) as function of time, temperature, and $\mathrm{pH}$. The variations observed can 

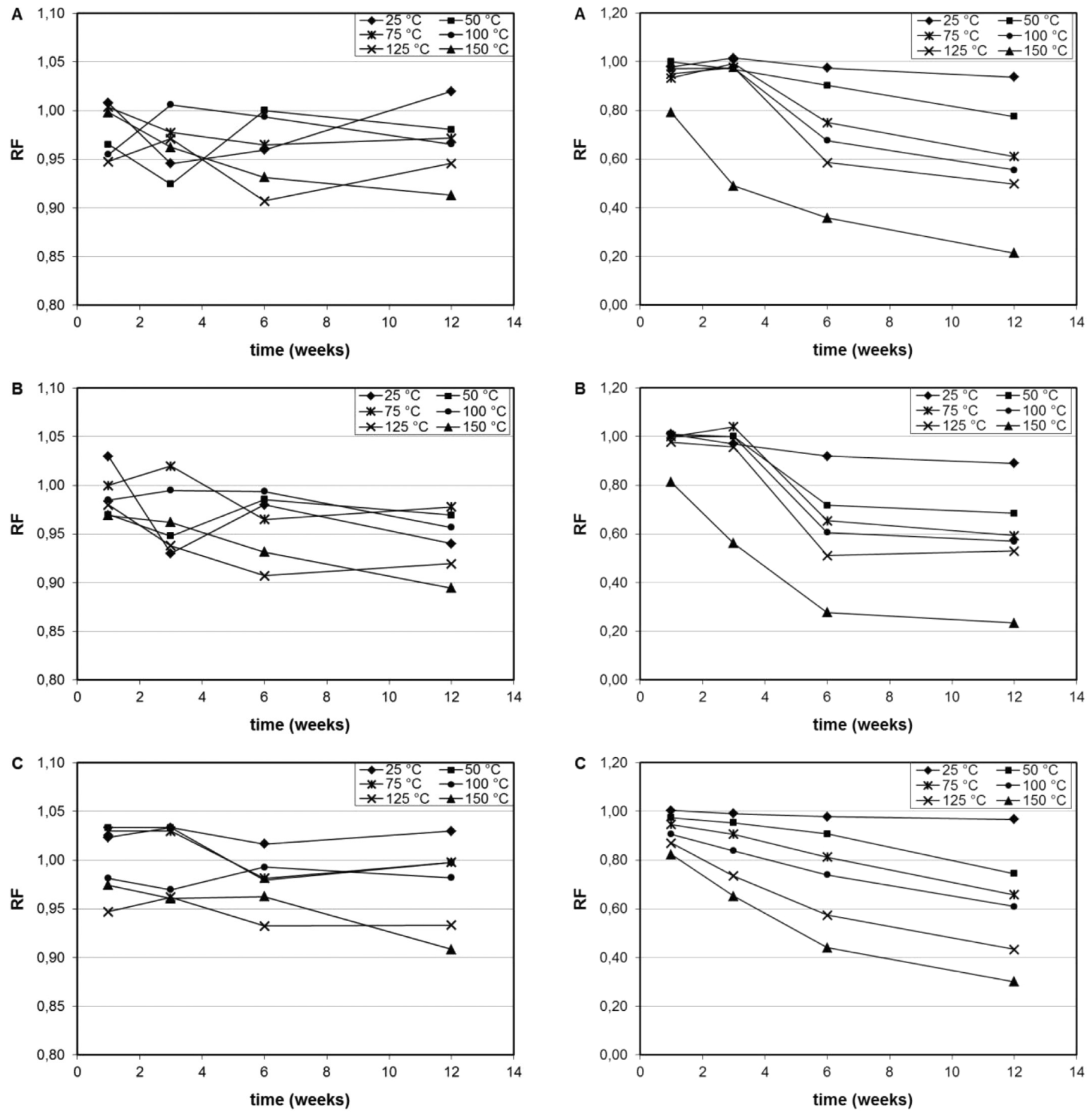

Fig. 1. RF of pyridine as function of time and temperature of incubation. (A) Initial pH 5.5; (B) Initial $\mathrm{pH} 7.1$; (C) Initial $\mathrm{pH} 8.0$ and no rock substrate in any of the samples.

be attributed to analytical uncertainty. Thus, results show that pyridine exhibits the stability required for use as PITT tracer. Mass conservation is critical for this application.

\subsection{2-Hydroxy-6-methylpyridine}

2-Hydroxy-6-methylpyridine (results in Fig. 2) is only stable at a temperature of $25{ }^{\circ} \mathrm{C}$. Unsuccessful attempts (not presented in this document) to develop a kinetic model for the degradation of 2-hydroxy-6methylpyridine were made.

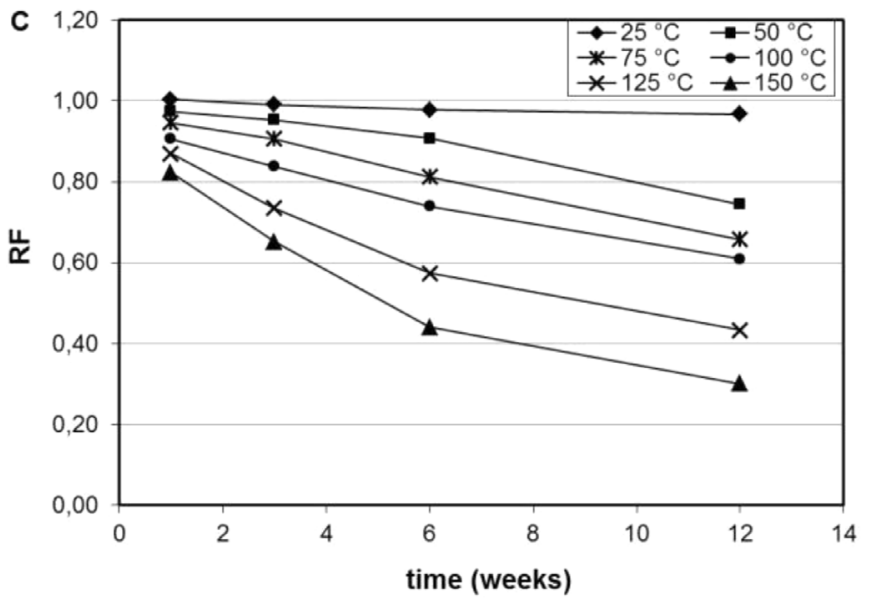

Fig. 2. RF of 2-hydroxy-6-methylpyridine as function of time and temperature of incubation. (A) Initial $\mathrm{pH} 5.5$; (B) Initial $\mathrm{pH} 7.1$; (C) Initial $\mathrm{pH} 8.0$ and no rock substrate.

The decrease in RF values of 2-hydroxy-6-methylpyridine observed at mild temperatures of $50{ }^{\circ} \mathrm{C}$ and $75{ }^{\circ} \mathrm{C}$ also suggests that this compound may be prone to microbial degradation. The rate of degradation of 2hydroxy-6-methylpyridine appears to be lower with increasing initial $\mathrm{pH}$ values. This effect is particularly noticeable for shorter incubation periods and higher temperatures. As the time of incubation increases, results suggest that temperature becomes the predominant driving force behind the degradation process, and influence of $\mathrm{pH}$ becomes less clear. Thus 2-hydroxy-6-methylpyridine is not an effective candidate to consider as PITT tracer. Furthermore, the difficulty in accurately describing 

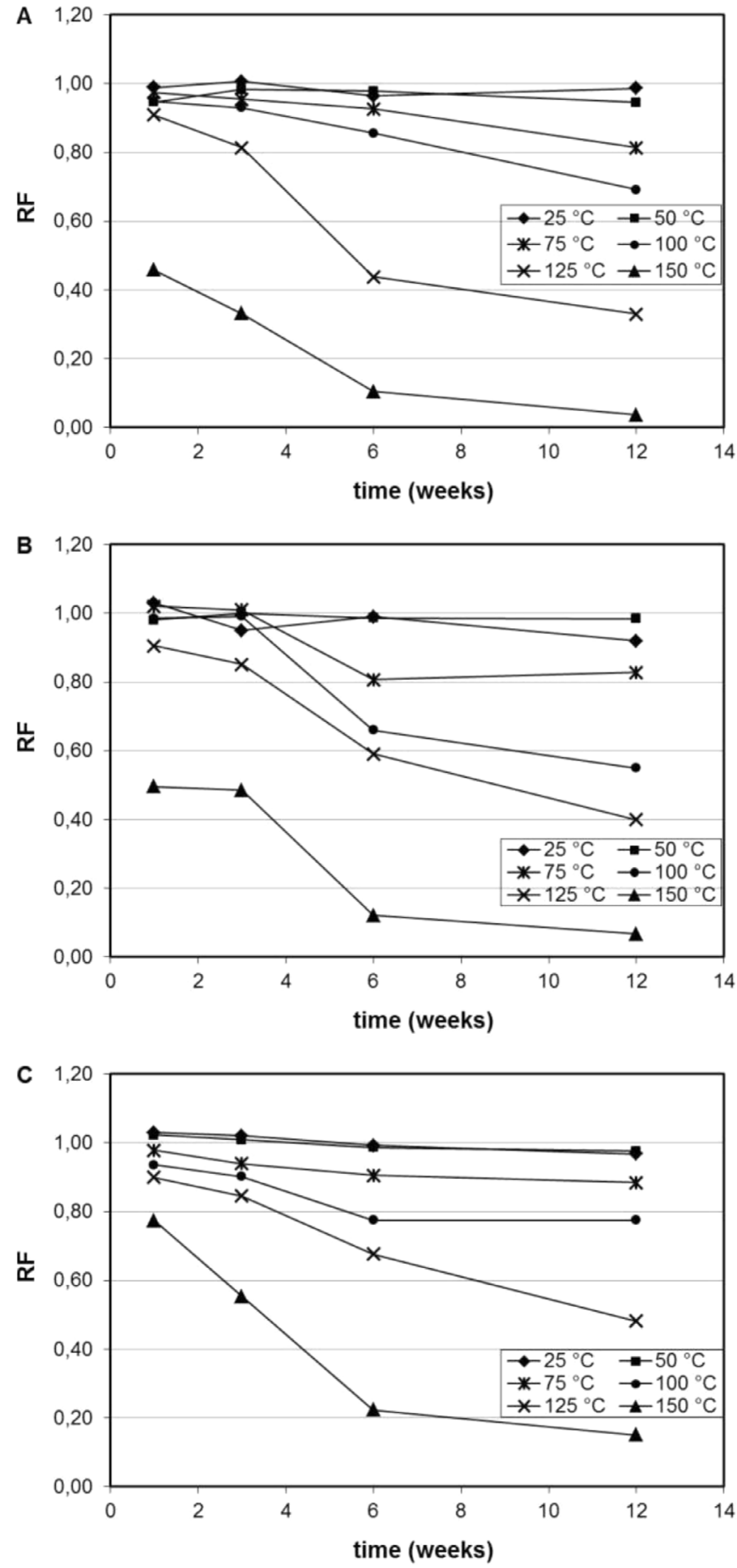

Fig. 3. RF of 3-Hydroxypyridine as function of time and temperature of incubation. (A) Initial pH 5.5; (B) Initial pH 7.1; (C) Initial pH 8.0 and no rock substrate.

the degradation of this compound leads us to conclude that it is most likely inappropriate for any use as tracer.

\subsection{3-Hydroxypyridine3-Hydroxypyridine}

Fig. 3 is stable at temperatures up to $75{ }^{\circ} \mathrm{C}$ and 12 weeks of thermal incubation. Microbial activity is unlikely at temperatures where degradation is observed. The use of 3-hydroxypyridine as mass conservative tracer could still be considered in low temperature reservoirs, typically onshore low depth ones, or in environmental applications such as as- sessment of near-surface contaminations by none-aqueous phase liquids (NAPL). Efforts to identify degradation products were unfruitful. The thermally driven degradation of 3-hydroxypyridine appears to be slower at higher $\mathrm{pH}$ values, however, this protective effect is not sufficient to compensate for the temperature effect. 3-Hydroxypyridine is unlikely to have any application as tracer in an oil reservoir.

\subsection{4-Methoxypyridine}

4-Methoxypyridine degrades at temperatures $\geq 75{ }^{\circ} \mathrm{C}$ with no influence from $\mathrm{pH}$ (Fig. 4). This suggest a thermally driven degradation mechanism.

The rate at which the degradation of 4-methoxypyridine occurs is not linear. Thus, is not zero order and is possibly concentration dependent. Further treatment of the variation of RF values of this compound was performed with the intention of assessing the accuracy of a first order kinetic degradation model in describing the experimental data. An accurate kinetic degradation model opens the possibility of using 4methoxypyridine as an active temperature probe to retrieve information about temperature or thermal fronts in reservoirs.

\subsection{Influence of rock substrate}

Three rock materials (Berea sandstone, limestone and kaolinite) were used as "model" reservoir rocks to evaluate interaction with the pyridines. These materials do not represent all the minerals that would be encountered in an oil basin, but they are representative of the most common sediment constituents and allow for the screening of a wide range of possible effects and interactions with the compounds investigated.

The maximum difference between $R F(t, T)$ values in the experiments with and without rock substrate encountered for each of the tested compounds with their $\pm 2 \sigma$ error bars are shown in Fig. 5 .

The maximum differences encountered for pyridine and 2-hydroxy6-methylpyridine are all within analytical uncertainty. These two compounds have no significant interaction with the rock materials and their use is not limited by the nature of the reservoir rocks.

$\mathrm{RF}$ values of 4-methoxypyridine are not significantly influenced by the presence of Berea sandstone and limestone, but dramatically influenced by the presence of kaolinite. In the presence of this clay, the maximum difference in $\mathrm{RF}$ values reaches about 0,77 . Thus, any field application considering this compound as a possible tracer should be performed with extreme caution. However, this also suggests that this compound can potentially be used to retrieve information about the presence and distribution of kaolinite and perhaps similar clays.

3-Hydroxypyridine is also insensitive to the presence of sandstone and carbonate rock materials and sensitive to kaolinite in a combined effect with pH. Fig. 5 clearly depicts a decrease in the maximum differences between RF in experiments with no rock substrate and experiments with kaolinite with increasing $\mathrm{pH}$ values.

Excluding the differences observed at $150{ }^{\circ} \mathrm{C}$, results in Fig. 6 show that there is a clear $\mathrm{pH}$ dependence on the interaction of 3hydroxypyridine with kaolinite.

The lower the $\mathrm{pH}$ of the experiment, the larger is the decrease in the concentration of 3-hydroxypyridine in the presence of kaolinite. The pKa value (Table 1) for 3-hydroxypyridinium (the protonated form of 3-hydroxypyridine) is 4.79 . Thus, as the $\mathrm{pH}$ decreases the concentration of the protonated species increases, which could make it more reactive with the interlayer ions in the kaolinite. The use of kaolinite as catalyst in reactions involving both organic and inorganic compounds has also been reported $[29,30]$. Thus, another possible explanation for the reduction of the RF of 3-hydroxypyridine with $\mathrm{pH}$ is that kaolinite is catalysing a reaction between 3-hydroxypyridinium and one of the metallic cations present in solution.

The possibility that kaolinite is adsorbing either the protonated form of 3-hydroxypyridine or its other possible products is not likely because 

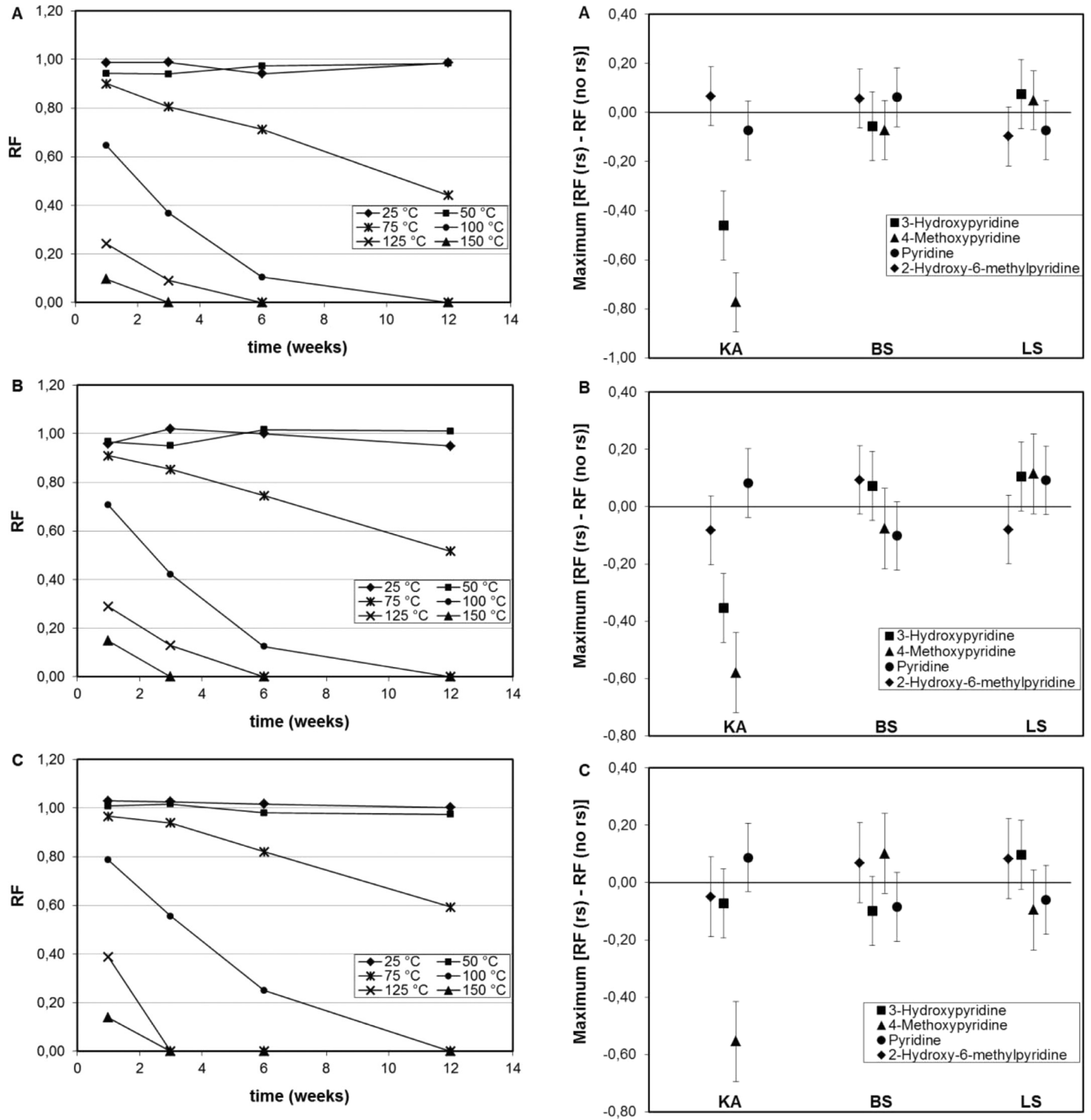

Fig. 4. RF of 4-methoxypyridine as function of time and temperature of incubation. (A) Initial pH 5.5; (B) Initial pH 7.1; (C) Initial pH 8.0 and no rock substrate.

the adsorption should be a function of temperature. Whatever the cause of reduction of the RF of 3-hydroxypyridine in the presence of kaolinite means that it will not survive long at detectable concentrations in reservoir sediments containing this clay.

\subsection{Kinetics of degradation of 4-methoxypyridine}

The thermal stability data of 4-methoxypyridine was analysed using a first order kinetic model. . Changes in $\mathrm{C}_{\mathrm{A}} / \mathrm{C}_{\mathrm{A} 0}(\mathrm{RF})$ at $75{ }^{\circ} \mathrm{C}, 100{ }^{\circ} \mathrm{C}$, $125{ }^{\circ} \mathrm{C}$, and $150{ }^{\circ} \mathrm{C}$ are shown in Fig. 7.

Fig. 5. Maximum differences encountered in RF values between experiments with rock substrate (rs) and experiments without rock substrate $\pm 2 \mathrm{x} \sigma$ in RF (t, T). (KA) kaolinite, (BS) Berea sandstone, (LS) limestone. (A) Initial pH 5.5; (B) Initial pH 7.1; (C) Initial pH 8.0.

The $\operatorname{Ln}\left(\mathrm{C}_{\mathrm{A}} / \mathrm{C}_{\mathrm{A} 0}\right)$ in Fig. 7 were linearly regressed as function of time of incubation. For first order kinetics, the apparent rate constant of the reaction ( $\mathrm{k}$ ) (weeks ${ }^{-1}$ ) at each temperature is the corresponding slope of the linear regression. With the linearised Arrhenius law $[k(T)$ as function of $1 / \mathrm{T}$ ] the apparent activation energy of the reaction and the preexponential factor (A) were obtained with the result presented in Eq. (1).

$\ln \left(\frac{C_{A}}{C_{A 0}}\right)=-1,86 \times 10^{7} \cdot e^{\frac{-56,4 \times 10^{3}}{R T}} \cdot t$ 


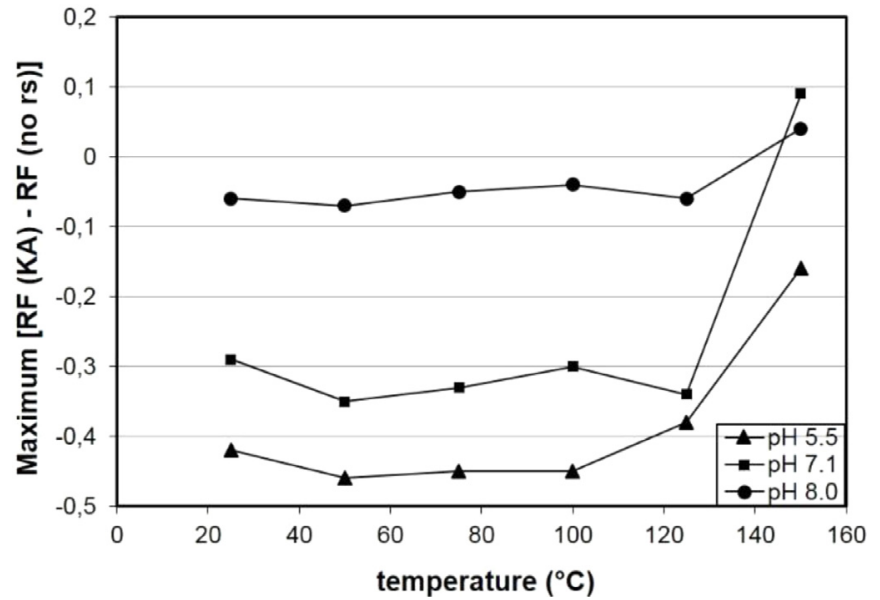

Fig. 6. Maximum differences encountered in RF values of 3-hydroxypyridine between experiments with kaolinite (KA) and experiments without rock substrate (rs) as function of temperature and $\mathrm{pH}$.

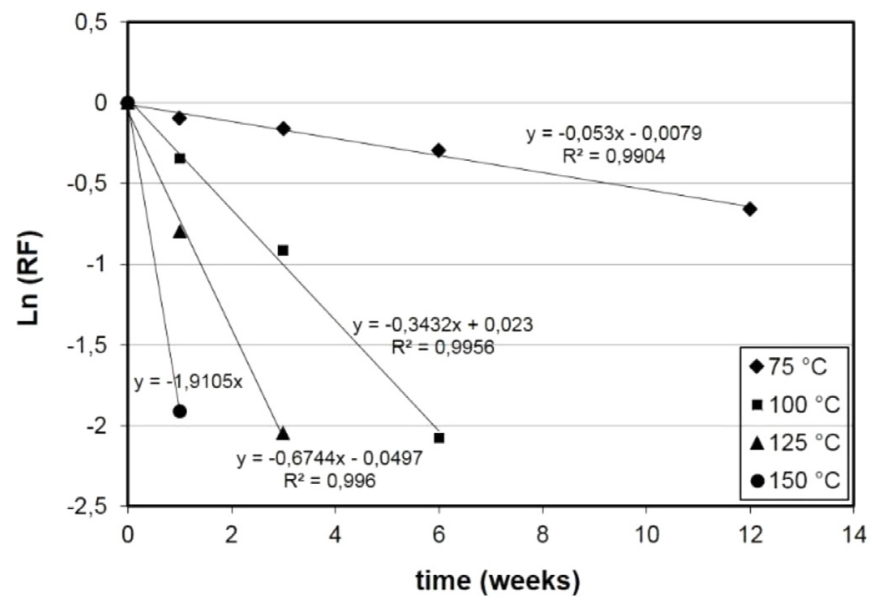

Fig. 7. Ln (RF) of 4-methoxypyridine as a function of time and linear regressions at each temperature. Initial experimental $\mathrm{pH} 7.1$, no rock substrate present.

The apparent activation energy of the reaction is $56,4 \mathrm{~kJ} / \mathrm{mol}$ and $A=1,86 \times 10^{7}$.

The accuracy of the model was tested by comparing its predicted RF values with the experimental ones. Two additional test samples (2.2) were prepared to obtain RF after 2 and 4 weeks of incubation. Experimental RF values $\pm 2 \sigma$ in their determination vs RF values predicted by the kinetic model are presented in Fig. 8.

\section{Conclusions}

The stability of pyridine, 2-hydroxy-6-methylpyridine, 3hydroxypyridine, and 4-methoxypyridine was evaluated at relevant reservoir temperatures $\left(25{ }^{\circ} \mathrm{C}\right.$ to $\left.150{ }^{\circ} \mathrm{C}\right), \mathrm{pH}(5,5 ; 7,1$ and 8,0$)$, with elevated salinity, and in the presence of sandstone, carbonate, and clay materials.

Results show that pyridine is not degraded during the 12 weeks of experiment at all tested temperatures. It is also insensitive to the presence of the rock materials and $\mathrm{pH}$ variations. Thus, pyridine appears to be fully stable and can be considered as an active (partitioning) mass conservative reservoir tracer. Since it has been reported as a constituent of crude oilsamples of produced waters from the field must be tested thoroughly prior to the use of this compound.

2-hydroxy-6-methylpyridine is only stable at $25{ }^{\circ} \mathrm{C}$ during the 12 weeks of testing. The stability of this compound is somewhat influenced

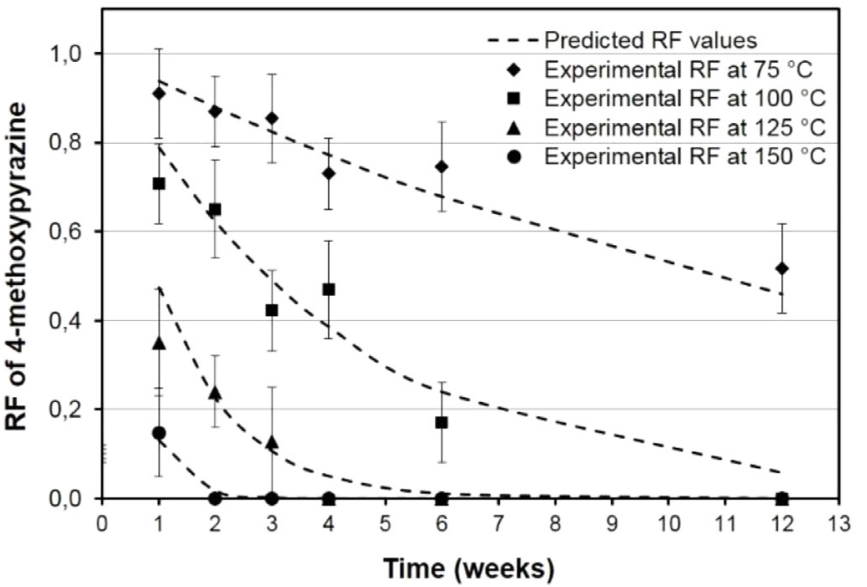

Fig. 8. Experimental vs predicted RF values of 4-methoxypyridine between 75 and $150{ }^{\circ} \mathrm{C}$. The uncertainty of $\pm 2 \sigma$ is not depicted for values under the lower analytical threshold $(\mathrm{RF}=0,05)$.

by $\mathrm{pH}$, becoming more stable at more basic values. This effect is more evident in experiments at high test temperatures and short incubation times. No significant interaction was observed between 2-hydroxy-6methylpyridine and any of the rock substrates. Results are inconclusive about the nature of the degradation phenomena, and microbial action cannot be excluded. Thus, any possible application based on this compound as tracer in the oilfield is highly unlikely.

3-Hydroxypyridine is fully stable up to $50{ }^{\circ} \mathrm{C}$ during 12 weeks of incubation. At higher temperatures, thermally driven degradation with influence from the $\mathrm{pH}$ conditions is observed. The rate of degradation decreases slightly as the $\mathrm{pH}$ increases. 3-Hydroxypyridine is insensitive to the presence of Berea sandstone and limestone, but a strong effect on its stability is observed under the presence of kaolinite in combination with $\mathrm{pH}$. Results suggest that kaolinite is most likely catalysing a reaction between the protonated species of this compound and a cation present in the bulk, as no temperature effect is observable which would suggest an adsorption phenomenon.

4-Methoxypyridine is stable for 12 weeks up to $50{ }^{\circ} \mathrm{C}$. Thermally driven degradation of this compound is observed at all other tested temperatures. A strong interaction with kaolinite causes a large decrease of the RF values of this compound in the whole range of temperatures tested. The nature of this interaction is uncertain, as no degradation products were identified. This tracer candidate is not affected by the presence of sandstone, limestone or $\mathrm{pH}$ variations (in the studied range) by themselves or in combination with other factors. The degradation of 4-methoxypyridine is well described by a pseudo first-order kinetic model without participation of other chemical species. The apparent activation energy and the pre-exponential factor of the Arrhenius equation of the thermal degradation were determined. The use of 4-methoxypyridine to retrieve thermal information from the reservoir is suggested.

\section{Declaration of Competing Interest}

The authors have no conflict of interests to declare.

\section{Acknowledgments}

The authors acknowledge the Research Council of Norway and the industry partners, ConocoPhillips Skandinavia AS, Aker BP ASA, Eni Norge AS, Maersk Oil, a company by Total, Statoil Petroleum AS, Neptune Energy Norge AS, Lundin Norway AS, Halliburton AS, Schlumberger Norge AS, Wintershall Norge AS, and DEA Norge AS, of The National IOR Centre of Norway for support. 


\section{References}

[1] R. Khaledialidusti, S. Enayatpor, S.J. Badham, C.T. Carlisle, J. Kleppe, An innovative technique for determining residual and current oil saturations using a combination of Log-Inject-Log and SWCT test methods: LIL-SWCT, J. Pet. Sci. Eng. 135 (2015) 618-625.

[2] O. Dugstad, S. Viig, B. Krognes, R. Kleven, O. Huseby, Tracer monitoring of enhanced oil recovery projects, in: A. Haugan (Ed.), Proceedings of the Sixth International Conference On Tracers and Tracing Methods, Tracer 6, E D P Sciences, Cedex A, 2013.

[3] A. Muggeridge, A. Cockin, K. Webb, H. Frampton, I. Collins, T. Moulds, P. Salino, Recovery rates, enhanced oil recovery and technological limits, Philos. Trans. A Math. Phys. Eng. Sci. 372 (2014) 20120320.

[4] C. Serres-Piole, H. Preud'homme, N. Moradi-Tehrani, C. Allanic, H. Jullia, R. Lobinski, Water tracers in oilfield applications: guidelines, J. Pet. Sci. Eng. 98-99 (2012) 22-39.

[5] C.E.J. Cooke, Method of Determining Fluid Saturations in Reservoirs" (US Patent 3,590,923), Esso Production Research Company, USA, 1971.

[6] M. Silva, H. Stray, T. Bjørnstad, Stability assessment of PITT tracer candidate compounds: the case of benzyl alcohols, J. Pet. Sci. Eng. 167 (2018) 517-523.

[7] M. Sanni, M. Al-Abbad, S. Kokal, Ø. Dugstad, S. Hartvig, O. Huseby, Pushing the envelope of residual oil measurement: a field case study of a new class of inter-well chemical tracers, J. Pet. Sci. Eng. 163 (2018) 538-545.

[8] M. Silva, H. Stray, T. Bjørnstad, Stability assessment of PITT tracer candidate compounds - The case of pyrazines, J. Pet. Sci. Eng. 182 (2019) 106269.

[9] S.O. Viig, H. Juilla, P. Renouf, R. Kleven, B. Krognes, O. Dugstad, O.K. Huseby, Application of a new class of chemical tracers to measure oil saturation in partitioning interwell tracer tests, in: Proceedings of the SPE International Symposium On Oilfield Chemistry, Society of Petroleum EngineersThe Woodlands, Texas, USA, 2013.

[10] M.C. Adams, J. Davis, Kinetics of fluorescein decay and its application as a geothermal tracer, Geothermics 20 (1991) 53-66.

[11] M. Schaffer, K.R. Idzik, M. Wilke, T. Licha, Amides as thermo-sensitive tracers for investigating the thermal state of geothermal reservoirs, Geothermics 64 (2016) 180-186.

[12] J.S. Tang, Propagation of phenol in aquifer with reversible adsorption, in: Proceedings of the Go-Expo Gas and Oil Exposition and the 4 Annual Canadian International Petroleum Conference and the 54 Annual Technical Meeting of the Petroleum Society of CIM: Global Challenges and Technology Integration, Petroleum Society of CIM, Canada, 2003.

[13] G.H.C. Prado, Y. Rao, A. de Klerk, Nitrogen removal from oil: a review, Energy Fuels 31 (2017) 14-36.

[14] P.J. King, F. Morton, A. Sagarra, Chemistry and physics of petroleum, in: G.D. Hobson, W. Pohl (Eds.), Modern Petroleum Technology, Applied Science Publishers, Barking, U.K., 1973.

[15] E. Bae, J.-.G. Na, S.H. Chung, H.S. Kim, S. Kim, Identification of about 30,000 chemical components in Shale Oils by Electrospray Ionization (ESI) and Atmospheric Pressure Photoionization (APPI) coupled with 15 T Fourier Transform Ion Cyclotron Resonance Mass Spectrometry (FT-ICR MS) and a comparison to conventional oil, Energy Fuels 24 (2010) 2563-2569.
[16] S. Shimizu, N. Watanabe, T. Kataoka, T. Shoji, A. Nobuyuki, S. Morishita, H. Ichimura, Pyridine and pyridine derivatives, Ullmann's Encyclopedia of Industrial Chemistry, Wiley-VCH, 2000.

[17] S.-m. Liu, C.-.H. Wu, H.-.J. Huang, Toxicity and anaerobic biodegradability of pyridine and its derivatives under sulfidogenic conditions, Chemosphere 36 (1998) 2345-2357.

[18] J. Shen, Y. Chen, S. Wu, H. Wu, X. Liu, X. Sun, J. Li, L. Wang, Enhanced pyridine biodegradation under anoxic condition: the key role of nitrate as the electron acceptor, Chem. Eng. J. 277 (2015) 140-149.

[19] H.M. Ahsan, B.K. Breedlove, S. Piangrawee, M.R. Mian, A. Fetoh, G. Cosquer, M. Yamashita, Enhancement of electrocatalytic abilities for reducing carbon dioxide: functionalization with a redox-active ligand-coordinated metal complex, Dalton Trans. 47 (2018) 11313-11316.

[20] S.Y. Desjardins, K.J. Cavell, J.L. Hoare, B.W. Skelton, A.N. Sobolev, A.H. White, W. Keim, Single component N-O chelated arylnickel(II) complexes as ethene polymerisation and $\mathrm{CO} /$ ethene copolymerisation catalysts. Examples of ligand induced changes to the reaction pathway, J. Organomet. Chem. 544 (1997) 163-174.

[21] F.A. Mautner, M. Traber, R.C. Fischer, K. Reichmann, R. Vicente, Synthesis and characterization of pseudohalide-metal(II) complexes with 4-methoxypyridine as co-ligand, Polyhedron 144 (2018) 30-35.

[22] K. Yamada, M. Brousseau, W. Honma, A. Iimura, H. Imase, Y. Iwaki, T. Kawanami, D. LaSala, G. Liang, H. Mitani, K. Nonomura, O. Ohmori, M. Pan, D.F. Rigel, I. Umemura, K. Yasoshima, G. Zhu, M. Mogi, Discovery of a novel piperidine-based inhibitor of Cholesteryl Ester Transfer Protein (CETP) that retains activity in hypertriglyceridemic plasma, J. Med. Chem. 60 (2017) 8466-8481.

[23] M.C. Gutiérrez, D. Carriazo, C.O. Ania, J.B. Parra, M.L. Ferrer, F. del Monte, Deep eutectic solvents as both precursors and structure directing agents in the synthesis of nitrogen doped hierarchical carbons highly suitable for $\mathrm{CO}_{2}$ capture, Energy Environ. Sci. 4 (2011) 3535-3544.

[24] D.-.H. Kim, A.Y. Sung, Preparation and characterization of ophthalmic polymer containing pyridine group and platinum nanoparticles with initiator behavior using photo and thermal polymerization, J. Nanosci. Nanotechnol. 18 (2018) 7027-7032.

[25] S. Kaviani, M. Izadyar, The possibility of iron chelation therapy in the presence of different HPOs; a molecular approach to the non-covalent interactions and binding energies, J. Mol. Struct 1166 (2018) 448-455.

[26] A. Chakraborty, J.E. Yarnell, R.D. Sommer, S. Roy, F.N. Castellano, Excited-state processes of Cyclometalated Platinum(II) charge-transfer dimers bridged by hydroxypyridines, Inorg. Chem. 57 (2018) 1298-1310.

[27] J.G. Małecki, J. Mroziński, K. Michalik, Structural, spectroscopic and magnetic properties of Mn(II), Co(II) and Ni(II) complexes with 2-hydroxy-6-methylpyridine ligand, Polyhedron. 30 (2011) 1806-1814.

[28] M. Silva, T. Bjørnstad, Determination of phase-partitioning tracer candidates in production waters from oilfields based on solid-phase microextraction followed by gas chromatography-tandem mass spectrometry, J. Chromatogr. A 1629 (2020) 461508.

[29] T.P. Vorlicek, G.R. Helz, Catalysis by mineral surfaces: implications for Mo geochemistry in anoxic environments, Geochim. Cosmochim. Acta 66 (2002) 3679-3692.

[30] T.J. Strathmann, A.T. Stone, Mineral surface catalysis of reactions between FeII and oxime carbamate pesticides, Geochim. Cosmochim. Acta 67 (2003) 2775-2791. 



\section{Paper VI}

Determination of phase-partitioning tracer candidates in production waters from oilfields based on solid-phase microextraction followed by gas chromatography-tandem mass spectrometry.

Mário Silva, Tor Bjørnstad (2020)

J. Chromatogr. A, (1629) 461508. DOI: 10.1016/j.chroma.2020.461508.

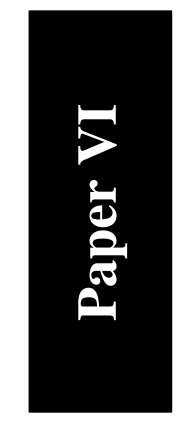




\title{
Determination of phase-partitioning tracer candidates in production waters from oilfields based on solid-phase microextraction followed by gas chromatography-tandem mass spectrometry
}

\author{
Mario Silva ${ }^{\mathrm{a}, \mathrm{b}, \mathrm{c}, *}$, Tor Bjørnstad ${ }^{\mathrm{a}, \mathrm{c}}$ \\ a The National IOR Centre of Norway, University of Stavanger, 4036 Stavanger, Norway \\ ${ }^{\mathrm{b}}$ Department of Energy Resources, University of Stavanger, 4036 Stavanger, Norway \\ ${ }^{\mathrm{c}}$ Institute for Energy Technology (IFE), Department of Tracer Technology, Instituttveien 18, 2007 Kjeller, Norway
}

\section{A R T I C L E I N F O}

\section{Article history:}

Received 22 July 2020

Revised 20 August 2020

Accepted 21 August 2020

Available online 22 August 2020

\section{Keywords:}

SPME

DI-HS

GC-MS/MS

PITT

Tracers

\begin{abstract}
A B S T R A C T
In the present document, we report the development of an analytical method consisting of a sequential direct-immersion/headspace solid-phase microextraction (DI-HS-SPME) followed by gas-phase chromatography and tandem mass spectrometry (GC-MS/MS) for simultaneous analysis of 4-chlorobenzyl alcohol, 2,6-dichlorobenzyl alcohol, 4-methoxybenzyl alcohol, 3,4-dimethoxybenzyl alcohol, pyridine, and 2,3-dimethylpyrazine in oilfield production waters. These compounds are under evaluation for use as phase-partitioning tracers in oil reservoirs. To the best of our knowledge, this is the first time SPME has been applied to the analysis of these compounds in production waters, or any other type of matrix where the compounds targeted are the base for a technical application. Relevant extraction parameters, such as the adsorbent phase of the fiber, direct immersion or headspace, addition of salt, temperature and time of extraction were investigated. The final optimal operation conditions consist on extracting 5 $\mathrm{mL}$ of sample at pH 9.0 with $1.8 \mathrm{~g}$ of $\mathrm{NaCl}$ with constant stirring during 5 minutes of DI-SPME followed by 15 minutes of HS-SPME at $70{ }^{\circ} \mathrm{C}$ using a DVB/CAR/PDMS $(50 / 30 \mu \mathrm{m})$ fiber. The limits of quantification (LOQ), linearity, precision and accuracy of the method were evaluated. Analyses of the tracer compounds and recovery studies were also performed on production waters from 8 different oilfields of the Norwegian continental shelf. LOQs between 0.080 and $0.35 \mu \mathrm{g} \mathrm{L}^{-1}$ were obtained. The recovery yields of the method were consistently higher than $85 \%$ and RSDs less than $13 \%$. None of the tracer compounds was found in the real samples processed, which is consistent with one of the requirements for an artificial tracer in an oilfield: absence or constant and low background in the traced fluid. The performance of the method developed, combined with its easiness to automate, introduce a new, accurate and cost-efficient technique to process the hundreds of samples required by an inter-well tracer test.
\end{abstract}

(c) 2020 The Authors. Published by Elsevier B.V.

This is an open access article under the CC BY license. (http://creativecommons.org/licenses/by/4.0/)

\section{Introduction}

Nowadays, tracer tests are routinely used by the oil industry to retrieve information about the reservoir. One type of such tests is the partitioning inter-well tracer test (PITT). A PITT measures the residual oil saturation $\left(\mathrm{S}_{\mathrm{OR}}\right)$ in the flow path between injectorproducer pairs in waterflooded oilfields [1-3]. $S_{O R}$ is an important parameter for the conception and evaluation of improved oil recovery projects (IOR) in mature oil reservoirs, where conventional recovery processes fail to mobilize the remaining reserves of hydrocarbons. The average hydrocarbon recovery in conventional oil

\footnotetext{
* Corresponding author.

E-mail addresses: mario.silva@ife.no, mariohsilva@sapo.pt (M. Silva).
}

reservoirs is lower than $50 \%$ when production is stopped and large unexplored basins are located in remote and/or environmentally sensitive areas [4]. At the same time, projections from the International Energy Agency (IEA) indicate an increase of the global demand for fossil hydrocarbons until the year 2040 [5]. Satisfying the global demand for hydrocarbons requires further and efficient exploration of mature oilfields. Thus, the number of IOR projects has consistently been growing as well as the number of PITT which provide the data for them [6]. A PITT consists of the simultaneous injection of at least one passive tracer and one oil/water partitioning tracer that will travel the same flow path inside the reservoir. The partitioning tracer will be delayed relatively to the passive one due to an equilibrium distribution between the nearly stagnant hydrocarbon phase and the flowing 
aqueous phase, and this delay is used to determine the hydrocarbon concentration $[7,8]$. The determination of the delay in the arrival of the partitioning tracers relies on the quantification of the tracer compounds often in several hundreds of produced water samples, collected during the test's timeframe, to build the tracer production curves. Low limits of quantification (LOQ) are desirable to increase the accuracy of these curves and also to reduce the amount of chemicals used in the test itself [9]. The growing attention on PITTs leads to a need for developing new oil/water partitioning tracers specifically qualified for this application in order to minimize the risk of unsuccessful field tests due to the use of inadequate tracer compounds [3,10]. 4-Chlorobenzyl alcohol; 2,6-dichlorobenzyl alcohol; 4-methoxybenzyl alcohol; 3,4dimethoxybenzyl alcohol; pyridine; and 2,3-dimethylpyrazine were identified as relevant compounds in an ongoing comprehensive R\&D project to introduce new oil/water partitioning tracers for the inter-well region of oil reservoirs $[3,11,12]$. Thus, an analytical method to identify and quantify these compounds in production waters from oilfields is required.

The analysis of organic compounds used as tracers in production waters from oil reservoirs is challenging and often requires several sample preparation steps. The sample preparation required to obtain acceptable LOQs typically involves an extraction/cleanup and concentration step using solid phase extraction (SPE), redissolution and, in some cases, derivatization [13,14] prior to analysis by GC-MS or LC-MS [15]. Although SPE is one of the most accepted and widely used sample preparation techniques [16], it is labor-intensive, time consuming and uses large amounts of solvents due to the high number of samples processed to complete the tracer test. In fact, the sample preparation step can be defined as the "bottleneck" of the analysis [17].

Solid-phase microextraction (SPME) is a mature, versatile, easy to automate, and solvent free sample preparation/concentration technique successfully used in a wide variety of applications with complex matrices, ranging from environmental analysis to clinical studies [18-20]. SPME fibers with several different adsorbent phases are commercially available. This is an advantage when considering the use of SPME in the processing of samples from PITTs. Standard and robust techniques are desirable to satisfy the large output of analysis required by the scope of this application. To the best of our knowledge, SPME has never been applied to the analysis of samples of production waters from oilfields. SPME has, however, been reported in the analysis of compounds from the same family of the compounds described in the present study. SPME was successfully used in the identification and quantification of benzyl alcohol [21-26], pyridine and pyridine derivatives [27-32] and substituted pyrazines [33-36] in aqueous, solid and gas matrices, and is also routinely used in the determination of volatile organic compounds in waste waters [37]. The extraction of analytes from aqueous samples using SPME is done either by direct immersion (DI-SPME) or by headspace extraction (HS-SPME). DI-SPME mode has been reported to be more efficient in the determination of less volatile oxygenated organic compounds leading, however, to a higher risk of fiber contamination or damage. HS-SPME mode is more indicated for more volatile analytes as it protects the fiber from such risks [37].

In the present study, we propose a methodology for analysis of the compounds identified as interesting PITT tracers in production waters from oilfields based on SPME-GC-MS/MS, with a sequential DI-SPME and HS-SPME extraction. This is the first report of the use of SPME in this matrix and we show that by introducing a sequential step of the two extraction modes (DI and HS), matrix interferences can be overcome and LOQs in the $\mathrm{ng} \mathrm{L}^{-1}$ range achieved. The method presented has the potential to significantly reduce the time, labor and solvents used in the analysis of tracers in production waters. The developed methodology was applied to target the tracer compounds in production waters from 8 different oilfields of the Norwegian continental shelf.

\section{Experimental}

\subsection{Materials and reagents}

4-Chlorobenzyl alcohol (99\%), 2,6-dichlorobenzyl alcohol (99\%), 4-methoxybenzyl alcohol (> 98\%), 3,4-dimethoxybenzyl alcohol (99\%), pyridine ( $\geq 99 \%$ ) and 2,3-dimethylpyrazine (99\%), manual SPME fiber holder, SPME fibers with coatings of CAR/PDMS (75 $\mu \mathrm{m})$, PDMS/DVB $(65 \mu \mathrm{m})$, DVB/CAR/PDMS $(50 / 30 \mu \mathrm{m})$, PA $(85 \mu \mathrm{m})$ and PDMS $(100 \mu \mathrm{m}), 10 \mathrm{~mL}$ SPME vials with aluminum screw caps with PTFE septa, and magnetic stirrers were purchased from Sigma-Aldrich (Sigma-Aldrich Norway AS, 0252 Oslo). Ultra-pure deionized water was obtained from tap water treated with a MilliQ Advantage A10 system (Millipore, Burlington, MA, USA).

\subsection{Instrumentation}

The present study used a Thermo Scientific Trace ${ }^{\mathrm{TM}} 1310$ gas chromatograph (Thermo Fischer Scientific, Waltham, MA, USA) equipped with a Restek Rtx®-5MS column (30 m X 0.25 mm X $0.25 \mu \mathrm{m}$ ) and coupled with a triple quadrupole mass spectrometer Thermo Scientific TSQ 8000 (Thermo Fischer Scientific, Waltham, MA, USA). The temperature program of the oven was as follows: initial temperature $50{ }^{\circ} \mathrm{C}$ kept for 3 minutes, followed by a ramp of $20^{\circ} \mathrm{C} / \mathrm{min}$ to $110{ }^{\circ} \mathrm{C}$, and another ramp of $15{ }^{\circ} \mathrm{C} / \mathrm{min}$ to $290{ }^{\circ} \mathrm{C}$, and finally 7 minutes at $290{ }^{\circ} \mathrm{C}$. Helium with a purity of $99.999 \%$ (Praxair Norway AS, 0663 Oslo) was used as carrier gas at a constant flow of $1 \mathrm{~mL} / \mathrm{min}$. The temperature of the injector was $250{ }^{\circ} \mathrm{C}$ and the temperatures of the ion transfer line and ion source were $290{ }^{\circ} \mathrm{C}$ and $320^{\circ} \mathrm{C}$, respectively. The injector was operated in splitless mode for 2 minutes returning to split mode after this time. The mass spectrometer (MS) was operated in electron impact (EI) ionization mode $(+70 \mathrm{eV})$ and selected reaction monitoring (SRM) was used to monitor specific transitions for each of the target compounds presented in Table 1 . The operation conditions of the MS were previously optimized for the target compounds.

\subsection{Field samples}

$1 \mathrm{~L}$ of production water samples from 8 different oilfields on the Norwegian continental shelf were obtained from the respective operators. The oilfields in question were as follows: Snorre A, Snorre B, Ekofisk M, Gullfaks C, Heidrun A, Eldfisk A, Eldfisk S and Vigdis B. These are fields close to maturity that have been under water flooding conditions for many years. Typical ranges for several physicochemical parameters of produced waters from oil reservoirs can be found in published literature [38].

\subsection{Experimental procedure}

\subsubsection{Selection of the type of SPME fiber and preliminary tests}

$1 \mathrm{~L}$ of individual solutions of each of the target compounds with a concentration of $10 \mu \mathrm{g} / \mathrm{L}$ were prepared in ultra-pure water. The $\mathrm{pH}$ of the solution was adjusted to $9.0 \pm 0.1$ to prevent protonation of the pyridine, with sodium hydroxide $0.05 \mathrm{M}$ and measuring the solution under constant stirring using a pH meter. $5 \mathrm{~mL}$ of solution were transferred to the SPME vials and $1.8 \mathrm{~g}$ of $\mathrm{NaCl}$ were added. The solution was stirred on a magnetic stirrer at $80 \mathrm{rpm}$ for a minimum of 5 minutes before thermal incubation. Increasing the salinity of the matrix is a well-known technique to facilitate the extraction of organic compounds in solution [39], however extractions without any added $\mathrm{NaCl}$ were also performed. All SPME fibers were conditioned prior to use according to the instructions of the 
Table 1

Experimental GC-MS/MS parameters for the target compounds and some physico-chemical properties.

\begin{tabular}{|c|c|c|c|c|c|c|c|c|}
\hline Compound & Acronym & $\mathrm{MW}^{\mathrm{a}}\left(\mathrm{g} \mathrm{mol}^{-1}\right)$ & $\mathrm{VP}^{\mathrm{b}, \mathrm{c}}$ (mTorr) & $\log \mathrm{k}_{\mathrm{ow}}{ }^{\mathrm{b}, \mathrm{d}}$ & $\begin{array}{l}\text { Ret. time } \\
(\mathrm{min})\end{array}$ & $\begin{array}{l}\text { MS/MS transitions } \\
\text { (identification) }\end{array}$ & $\begin{array}{l}\text { MS/MS transitions } \\
\text { (quantification) }\end{array}$ & $\mathrm{CE}(\mathrm{eV})$ \\
\hline Pyridine & PYR & 79.04 & $19.3 \times 10^{3}$ & 0.65 & 5.89 & $\begin{array}{l}52 \rightarrow 39 \\
79 \rightarrow 52\end{array}$ & $79 \rightarrow 52$ & 30 \\
\hline 2,3-Dimethylpyrazine & 23MPRZ & 108.07 & $2.74 \times 10^{3}$ & 0.54 & 6.41 & $\begin{array}{l}40 \rightarrow 39 \\
67 \rightarrow 52 \\
108 \rightarrow 93\end{array}$ & $108 \rightarrow 93$ & 25 \\
\hline 4-Chlorobenzyl alcohol & $4 \mathrm{BZOH}$ & 142.02 & 0.268 & 0.82 & 14.13 & $\begin{array}{l}77 \rightarrow 75 \\
107 \rightarrow 90 \\
142 \rightarrow 125\end{array}$ & $142 \rightarrow 125$ & 20 \\
\hline 2,6-Dichlorobenzyl alcohol & $26 \mathrm{BZOH}$ & 175.98 & 0.254 & 1.07 & 14.82 & $\begin{array}{l}113 \rightarrow 77 \\
141 \rightarrow 123 \\
176 \rightarrow 159\end{array}$ & $113 \rightarrow 77$ & 20 \\
\hline 4-Methoxybenzyl alcohol & 4METBZOH & 138.07 & 0.082 & 0.71 & 16.47 & $\begin{array}{l}109 \rightarrow 95 \\
121 \rightarrow 90 \\
138 \rightarrow 107\end{array}$ & $138 \rightarrow 107$ & 25 \\
\hline 3,4-Dimethoxybenzyl alcohol & 34METBZOH & 168.08 & 0.0696 & 0.62 & 17.27 & $\begin{array}{l}139 \rightarrow 95 \\
151 \rightarrow 120 \\
168 \rightarrow 137\end{array}$ & $168 \rightarrow 137$ & 25 \\
\hline
\end{tabular}

\footnotetext{
a molecular weight.

b properties calculated using the US Environmental Protection Agency's EPISuite ${ }^{\mathrm{TM}}$.

c vapor pressure at $25^{\circ} \mathrm{C}$.

d octanol/water partition coefficient.
}

manufacturer. The extraction with the different SPME fibers was performed manually under constant stirring at a fixed temperature of $50{ }^{\circ} \mathrm{C}$ for 30 minutes, both in direct immersion and headspace modes. Three replicas were used for each test standard. The mean value of the chromatographic areas was used to choose both the most appropriate adsorbent phase and SPME extraction mode.

\subsubsection{Optimization of the conditions of SPME extraction}

A sample of real production water (Ekofisk M) was used to optimize this approach together with the selected SPME fiber. This sample was selected because it presented the highest contamination of hydrocarbons upon visual inspection. The sample was spiked with a mixture of the tracer compounds $(4 \mathrm{BZOH}, 26 \mathrm{BZOH}$, 4METBZOH, 34METBZOH, PYR, and 23MPRZ) at a concentration of $10 \mu \mathrm{g} / \mathrm{L}$. The $\mathrm{pH}$ was adjusted to $9.0 \pm 0.1$ and $5 \mathrm{~mL}$ were transferred into SPME vials. Again, constant stirring was used during the whole extraction period. The time and temperature of extraction were evaluated between $5-40$ minutes and $30-90{ }^{\circ} \mathrm{C}$, respectively, as well as different periods of DI and HS combined. This procedure intended to maximize the signal obtained from the analysis of the analytes while simultaneously preventing interference effects from the matrix. DI should increase the extraction yield of the compounds with lower volatility (4METBZOH, 34METBZOH). After the extraction and before insertion in the injector port, the SPME fiber was conditioned during 2 minutes in ultra-pure water to preserve the chromatographic system. The desorption time for the fiber in the injector port was set to 10 minutes.

\subsubsection{Validation of the method}

Using the optimized conditions of the method, limits of quantification and detection (LOQ and LOD) were determined at the concentration level for a signal to noise ratio $(\mathrm{S} / \mathrm{N})$ of 10 and 3 , respectively. The linearity was evaluated from the coefficient of determination by preparing a calibration curve and using $\mathrm{r} \geq 0.995$. To validate the obtained range, a standard residual analysis was performed as described by Eurachem [40]. The recovery was evaluated in all the 8 different available samples, spiked with known amounts of the analytes, and calculated using Eq. 1

$\%$ Recovery $=\frac{\text { Determined analyte concentration }}{\text { Expected analyte concentration }} \cdot 100$

Intra-day and inter-day precision were evaluated at 3 different concentration levels (low range, middle range, and high range) with 7 and 5 replicates per level for intra-day and inter-day, respectively.

\section{Results and discussion}

\subsection{Selection of the adsorbent SPME phase and preliminary tests}

The fixed conditions of time and temperature of extraction described in Section 2.4.1. allowed for a direct comparison between extraction modes (DI and HS) and to evaluate the impact of increased salinity on the efficiency of extraction, often described as key factor when SPME is used as sample preparation technique. For these tests, standard solutions of the individual target compounds in deionized water were used and the resulting average chromatographic areas $(\mathrm{n}=3)$ for each compound are presented in Fig. 1. Extraction of every compound in both DI-SPME and HSSPME modes was observed using 3 adsorbent phases (PDMS/DVB, CAR/PDMS and DVB/CAR/PDMS).

Results indicate that the PA SPME fiber fails to extract PYR, 23MPRZ, 4METBZOH and 34METBZOH in both DI and HS modes, while the PDMS fiber fails to extract 4METBZOH and 34METB$\mathrm{ZOH}$ in HS mode. The target analytes have a significant affinity for lipophilic phases, as deduced from their log $\mathrm{K}_{\mathrm{OW}}$ values (see Table 1). Polyacrylate is a linear polymer with polar groups. The polar interactions from these groups are likely not strong enough to disrupt the interactions between the water molecules and induce the partitioning of PYR, 23MPRZ, 4METBZOH and 34METB$\mathrm{ZOH}$ to the adsorbent phase, without the presence of a highly lipophilic chain. Thus, this is the possible reason why the PA SPME fiber fails to extract PYR, 23MPRZ, 4METBZOH and 34METBZOH. DI extraction mode improves the response relatively to HS for every compound and fiber used. This is particularly observable for 4METBZOH and 34METBZOH, the compounds with lower volatilities (see Table 1), and the global results are in general agreement with what could be expected when this property of the target analytes is considered, as they will be available for adsorption to the fiber in lower amounts in the headspace. The addition of $1.8 \mathrm{~g}$ of $\mathrm{NaCl}$ has a positive effect on the efficiency of extraction in both DI and HS modes for all the analytes (see Fig. 1). Improvements in the efficiency of extraction are particularly observed for the chlorinated benzyl alcohols, whose volatility is significantly impacted by the salinity of the aqueous matrix. The salting-out effect makes 


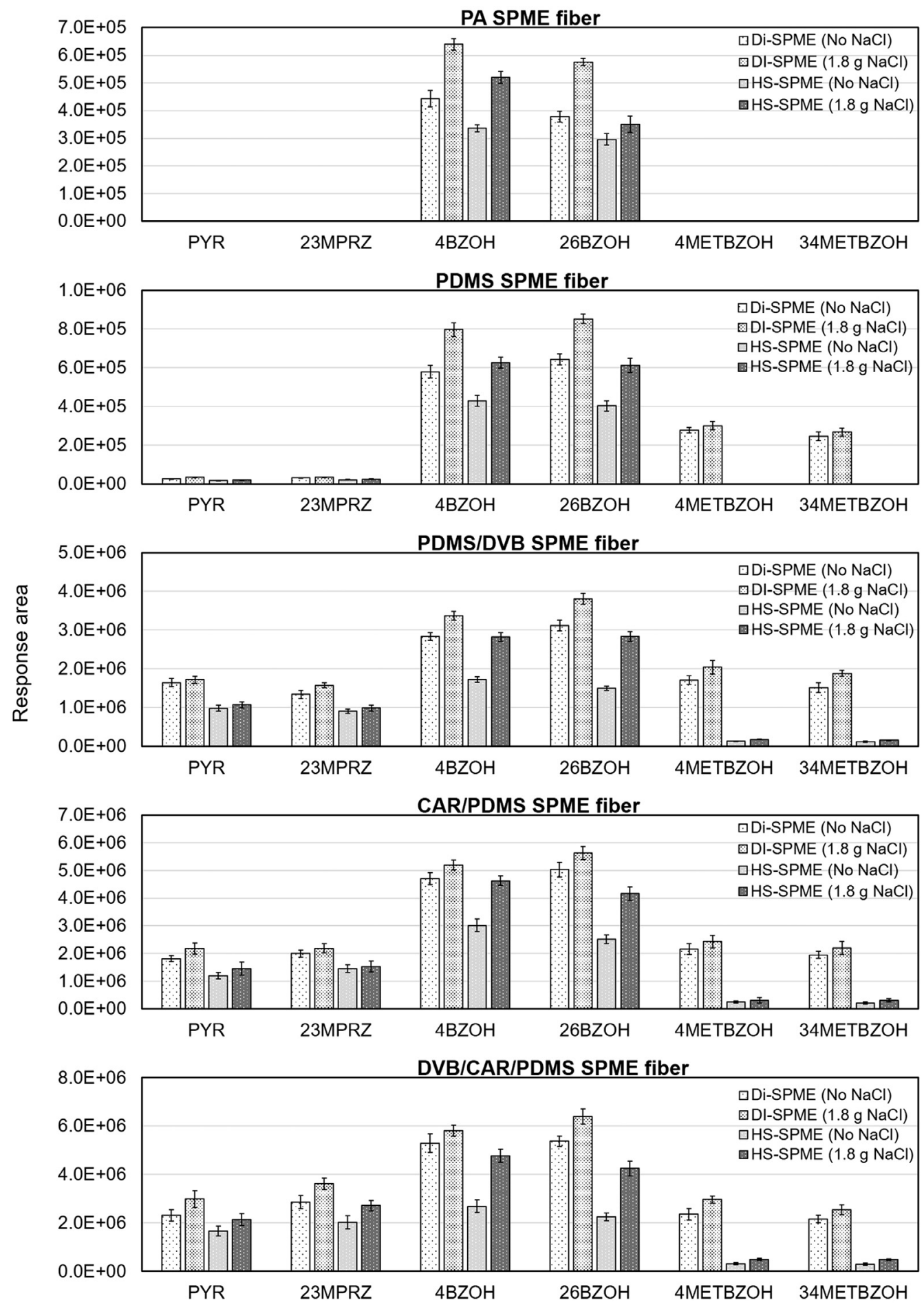

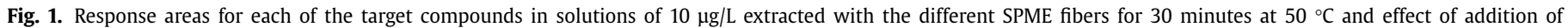
$\mathrm{NaCl}$.

them available in higher concentration in the vapor phase. The SPME fiber with the adsorbent phase DVB/CAR/PDMS produced the best results (slightly larger peak areas than CAR/PDMS) for every analyte in both extraction modes and was therefore selected for the rest of the study. Both the DVB/CAR/PDMS and CAR/PDMS fibers are often referred to as a "bi-polar" adsorbent phases, as they contain polar groups and non-polar groups. The possibility of polar and non-polar interactions with the analytes, increases their partitioning to the SPME fiber, resulting in higher efficien- cies of extraction. The fact that DVB/CAR/PDMS yields better results is likely due to an additional partition effect induced on the analytes by the benzene rings present on coating of the fiber. All the target compounds of the present study have either benzene rings or benzene-like cyclic structures. A DI-SPME extraction mode combined with the addition of $1.8 \mathrm{~g}$ of $\mathrm{NaCl}$ was initially considered, as the preliminary results suggested this approach to maximize the efficiency of extraction of all the 6 target tracer compounds. 


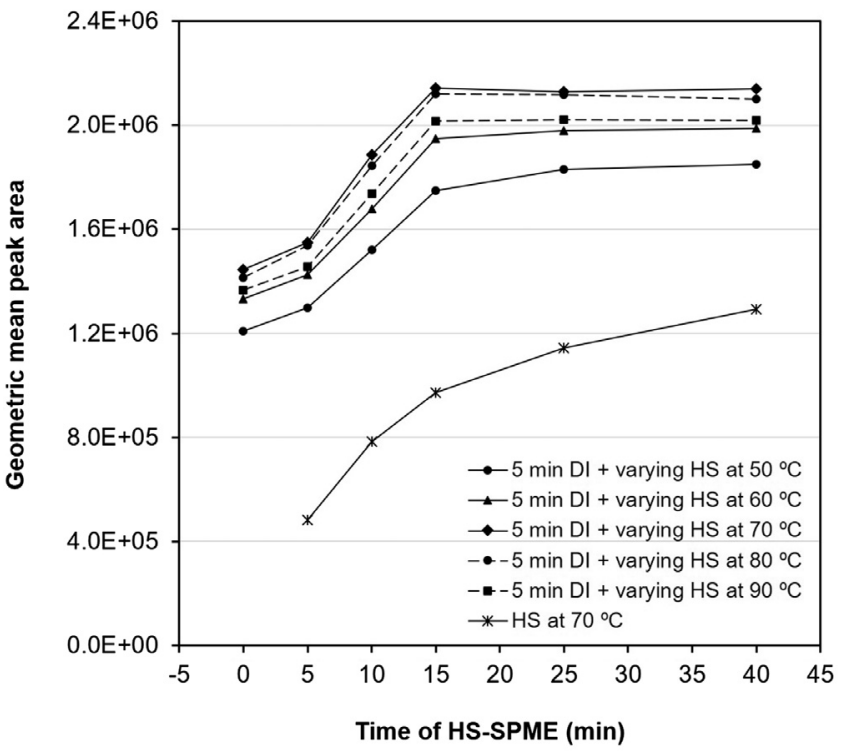

Fig. 2. Geometric mean response area of the target compounds as function of the time and temperature of HS-SPME extraction after a fixed DI-SPME period of 5 minutes.

\subsection{Optimization of the conditions of SPME extraction}

The study to optimize the temperature and time conditions for a DI-SPME extraction with the DVB/CAR/PDMS fiber was initiated on a mixed standard solution of all 6 compounds in deionized water with a concentration of $10 \mu \mathrm{g} / \mathrm{L}$. Because real production waters are complex matrices, tests were made to assess their influence on the performance of the extraction. We found that interferents could compromise the detectability of the compounds when prolonged DI extraction periods were used. If purely HS extraction was employed, the efficiency of extraction, particularly of the less volatile compounds (4METBZOH and 34METBZOH) would, on its turn, be severely reduced. Thus, a sequential DI-HS extraction was considered, and the maximum DI extraction time was evaluated to maximize the signals of the analytes without compromising their detectability due to interferences. An aliquot of the 8 different oilfield production waters was spiked with $10 \mu \mathrm{g} / \mathrm{L}$ of the tracer compounds and DI-SPME was performed for different periods of time. We verified that in the worst-case scenario (production water from Ekofisk M), about 6.5 minutes of DI-SPME would start compromising the detectability of the tracers. A sequential DI-HS-SPME extraction procedure with a fixed time of DI-SPME of 5 minutes was adopted to maximize as much as possible the measured responses for the analytes while simultaneously eliminating the risks of matrix interference. The time of HS-SPME was then optimized together with the temperature of extraction using production water from Ekofisk M spiked with $10 \mu \mathrm{g} / \mathrm{L}$ of the tracer compounds and with $1.8 \mathrm{~g}$ of $\mathrm{NaCl}$ added. Fig. 2 presents the geometric means of the areas of the compounds of interest as function of temperature and time of HS-SPME (with a fixed DI-SPME period of 5 minutes).

Results indicate that the maximum extraction efficiency is achieved with 15 minutes of HS-SPME extraction performed after 5 minutes DI-SPME at $70{ }^{\circ} \mathrm{C}$. The response areas obtained at this temperature are very similar to the ones obtained at $80{ }^{\circ} \mathrm{C}$ and an argument can be made that there are no significant differences between them. Because the results are so similar at these two temperatures and a slightly more elevated trend of values can be argued for in the results at $70{ }^{\circ} \mathrm{C}$, this was the temperature adopted for the rest of the study. SPME is not an exhaustive ex- traction technique and the equilibrium of the system was achieved after a relatively short period of 20 minutes ( 5 minutes of DI +15 minutes of HS) of extraction (except when extraction is done at 50 $\left.{ }^{\circ} \mathrm{C}\right)$. This is most likely due to a combined effect of temperature and the initial step being DI-SPME. Initiating the sequential extraction procedure with DI maximizes the mass transferring gradient between the bulk of the sample and the SPME fiber, thus maximizing the velocity of adsorption. Increasing temperature will increase the volatility of the analytes and promote their fastest transfer to the headspace once the equilibrium between it and the bulk of the sample is disturbed by the HS-SPME extraction. It should be noted that this is true until an upper temperature value. The results show that the efficiency of the SPME extraction decreases at a temperature of $90{ }^{\circ} \mathrm{C}$. This can be explained by the reduction of the adsorption capacity of the SPME fiber as consequence of an excessively high temperature of operation which promotes some desorption.

The final optimized SPME extraction procedure was as follows: $1.8 \mathrm{~g}$ of $\mathrm{NaCl}$ were added to an aliquot of $5 \mathrm{~mL}$ of sample at $\mathrm{pH} 9.0$ \pm 0.1 . The sample was kept under constant stirring and extracted at $70{ }^{\circ} \mathrm{C}$ with a sequential DI-HS-SPME consisting of 5 minutes of DI and 15 minutes of HS.

\subsection{Chromatographic analysis}

SPME extracts many other compounds from the real produced water samples in addition to the target analytes. Fig. 3 shows a total ion chromatogram (TIC) $(35-800 \mathrm{~m} / \mathrm{z})$ recorded from the sample of produced water from Ekofisk $\mathrm{M}$ spiked with the tracer compounds at a concentration of $10 \mu \mathrm{g} \mathrm{L}^{-1}$.

The result is a fairly complex chromatogram where the identification of the tracers is not clear at first sight, however the major ions of the EI spectra of all compounds may still be identified at their respective retention times (see Table 1). Using the triple quadrupole under tandem/MS conditions allows optimizing selectivity and sensitivity reducing the noise in the measured responses. Additionally, timed data acquisition was used to further enhance these parameters. The MS was operated in selected reaction monitoring (SRM) mode and three transitions per compound were monitored to ensure the identification of the analytes in combination with the respective chromatographic retention times. These were further used to define the periods for data acquisition by the MS. The operating conditions of the GC and the MS were previously optimized and information about the chromatographic retention times, collision energies, and transitions for identification and quantification are presented in Table 1. Fig. 4 displays a reconstructed SRM chromatogram obtained from a sample of production water from Ekofisk $M$ spiked with the tracers at a concentration of $1 \mu \mathrm{g} \mathrm{L} \mathrm{L}^{-1}$ and extracted with the optimized senquential DI-HSSPME procedure.

\subsection{Evaluation of the performance of the method}

The linearity, precision, accuracy, and recovery of the DI-HS-GCMS/MS method were evaluated. The limits of quantification (LOQ) and limits of detection (LOD) were calculated as the concentration of the compounds originating a signal to noise ratio $(\mathrm{S} / \mathrm{N})$ of 10 and 3, respectively, by applying the optimized analytical method to real samples spiked at varying low concentrations. We verified that the concentrations of the analytes which originate $S / N \geq 10$ are systematically lower than the lower linear concentration threshold. LOQ and LOD values are indicated in Table 2. Because the present method is conceived for an application where the processing of a large number of samples is required, the use of the lower limits of linearity as LOQ is recommended for systematic analysis. 


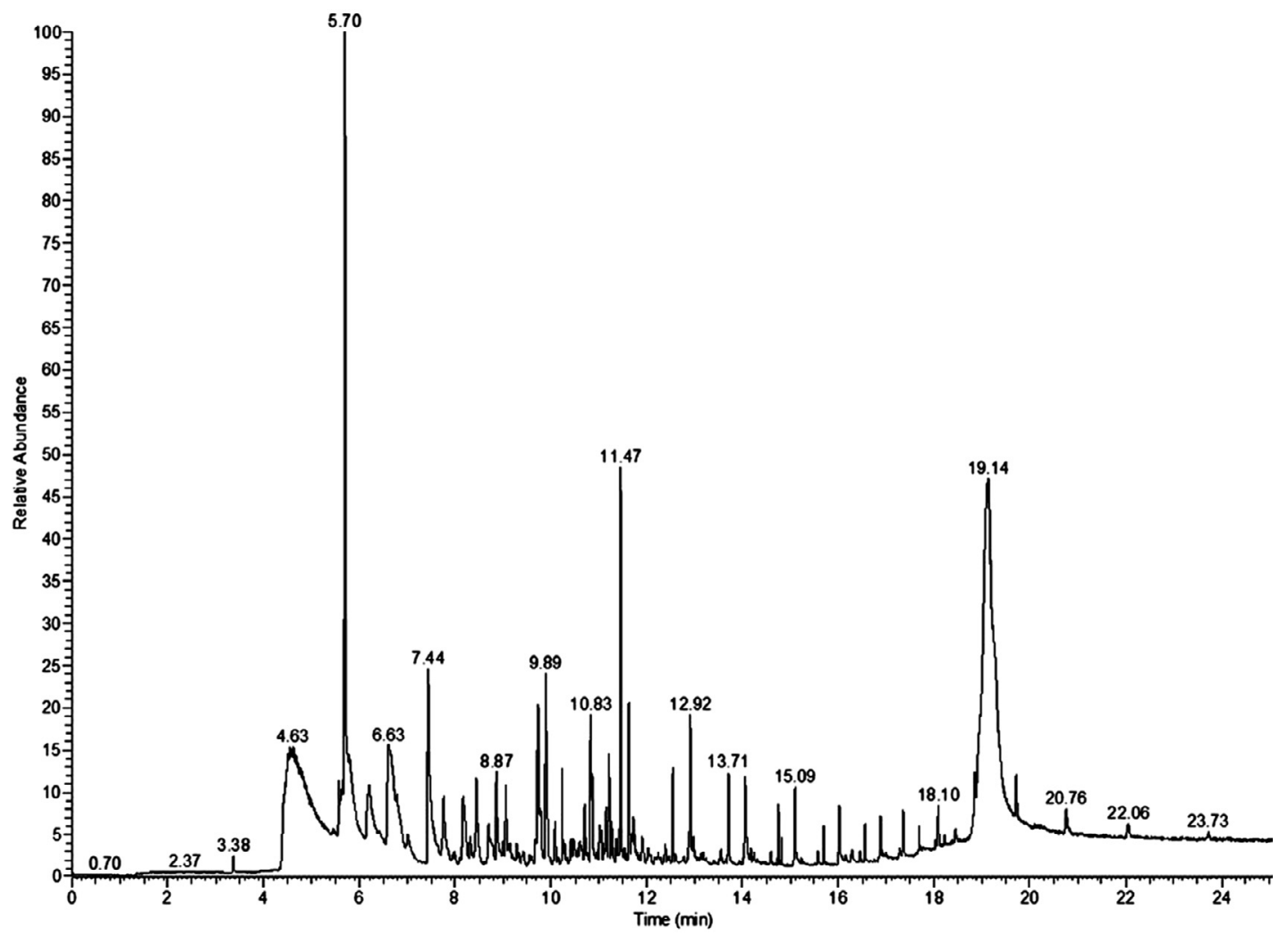

Fig. 3. TIC of produced water from Ekofisk M spiked at $10 \mu \mathrm{g} \mathrm{\textrm {L } ^ { - 1 }}$ with all 6 target compounds

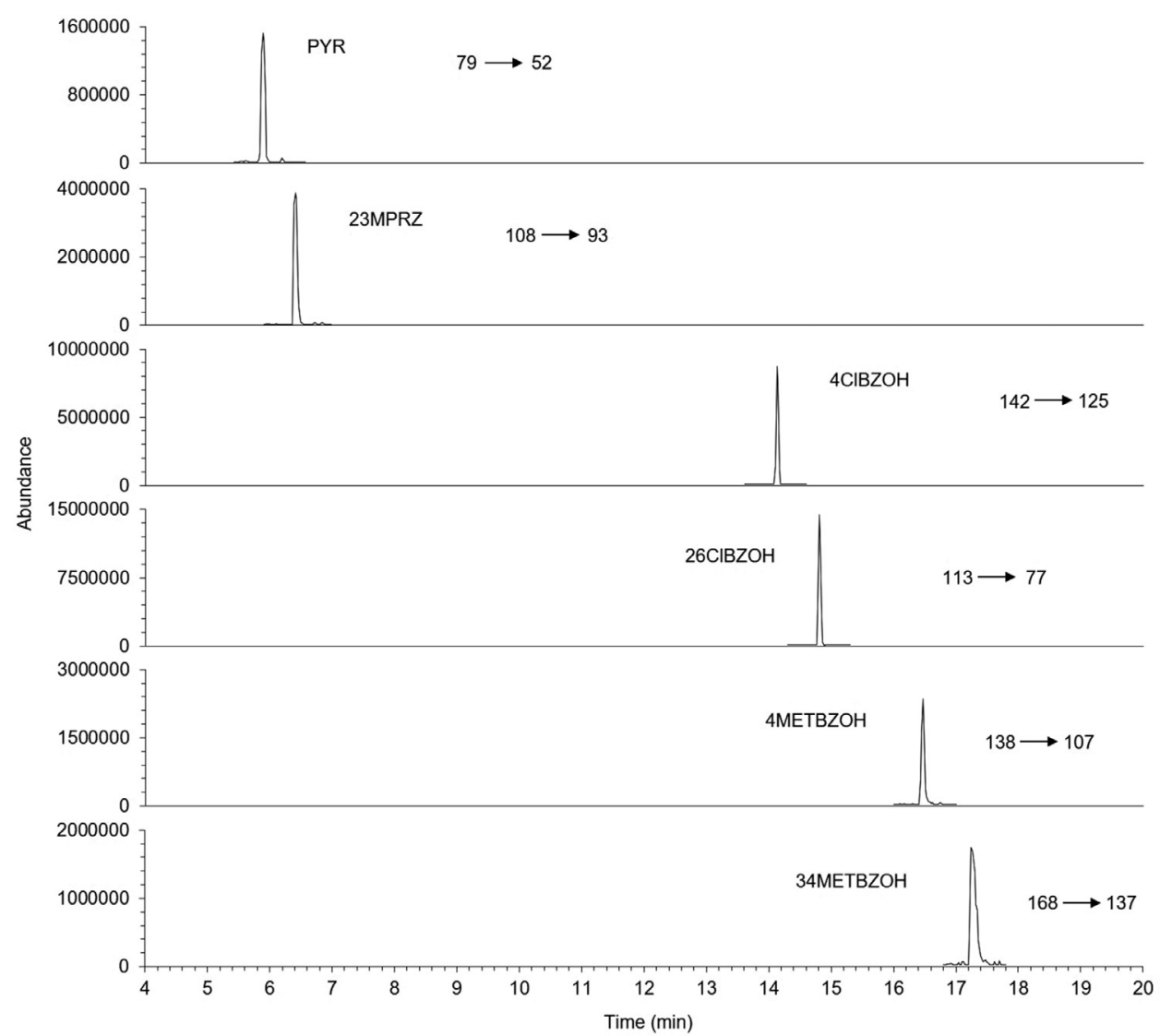

Fig. 4. Reconstructed SRM chromatogram of produced water from Ekofisk M spiked at $1 \mu \mathrm{g} \mathrm{L} \mathrm{L}^{-1}$ with all 6 target compounds. 
Table 2

Linearity, limits of quantification and precision achieved with the developed method.

\begin{tabular}{|c|c|c|c|c|c|c|c|c|c|}
\hline \multirow[t]{3}{*}{ Tracers } & & & \multirow[b]{3}{*}{$\operatorname{LoQ}^{a}$} & \multicolumn{6}{|c|}{ Precision (\% RSD) } \\
\hline & \multicolumn{2}{|l|}{ Linearity } & & \multicolumn{3}{|c|}{ Intra-day precision } & \multicolumn{3}{|c|}{ Inter-day precision } \\
\hline & Range $^{a}$ & $\mathrm{R}^{2}$ & & $0.50^{\mathrm{a}}$ & $2.5^{\mathrm{a}}$ & $10^{\mathrm{a}}$ & $0.50^{\mathrm{a}}$ & $2.5^{\mathrm{a}}$ & $10^{\mathrm{a}}$ \\
\hline PYR & $0.50-10$ & 0.9995 & 0.35 & 7.9 & 6.6 & 6.2 & 9.4 & 7.4 & 7.3 \\
\hline 23MPRZ & $0.30-50$ & 0.9970 & 0.20 & 4.7 & 5.3 & 4.2 & 7.1 & 7.2 & 5.3 \\
\hline 4BZOH & $0.10-20$ & 0.9962 & 0.08 & 8.2 & 7.0 & 4.7 & 10 & 8,5 & 5.7 \\
\hline $26 \mathrm{BZOH}$ & $0.10-20$ & 0.9971 & 0.10 & 6.2 & 7.1 & 4.2 & 8.1 & 7.8 & 5.6 \\
\hline 4METBZOH & $0.40-25$ & 0.9974 & 0.25 & 9.4 & 7.4 & 5.9 & 12 & 9.1 & 5.4 \\
\hline 26METBZOH & $0.40-25$ & 0.9984 & 0.25 & 10 & 6.4 & 3.9 & 12 & 11 & 7.5 \\
\hline
\end{tabular}

a $\mu g \mathrm{~L}^{-1}$.

Table 3

Recoveries (\%) and RSD (\%) of the DI-HS-SPME-GC-MS/MS method in 8 real oilfield production waters spiked at $0.50 \mu \mathrm{g} \mathrm{L}^{-1}$.

\begin{tabular}{|c|c|c|c|c|c|c|c|c|}
\hline \multirow[t]{2}{*}{ Tracers } & \multicolumn{8}{|c|}{ \%Recovery (\%RSD) - samples spiked at $0.50 \mu \mathrm{g} \mathrm{L}^{-1}$} \\
\hline & Snorre A & Snorre B & Ekofisk M & Gullfaks C & Heidrun A & Eldfisk A & Eldfisk S & Vigdis B \\
\hline PYR & $94.7(7.8)$ & $99.3(8.1)$ & $104(5.7)$ & $114(6.6)$ & $104(8.7)$ & $93.3(7.9)$ & $97.3(6.4)$ & $97.3(8.3)$ \\
\hline 23MPRZ & $105(5.4)$ & $98.7(3.4)$ & $95.3(6.9)$ & $94.0(5.2)$ & $97.3(6.8)$ & $95.3(13)$ & $103(9.2)$ & $108(4.0)$ \\
\hline 4BZOH & $97.3(5.9)$ & $90.0(8.3)$ & $86.7(7.8)$ & $101(5.7)$ & $96.7(4.3)$ & $101(11)$ & $99.3(8.1)$ & $100(4.9)$ \\
\hline $26 \mathrm{BZOH}$ & $101(5.7)$ & $91.3(5.5)$ & $103(5.6)$ & $93.3(5.3)$ & $98.0(6.7)$ & $96.7(11)$ & $97.3(12)$ & $101(11)$ \\
\hline 4METBZOH & $90.0(4.8)$ & $92.7(9.0)$ & $94.7(6.1)$ & $107(6.9)$ & $87.3(4.7)$ & $89.3(6.4)$ & $98.0(9.3)$ & $96.7(5.2)$ \\
\hline 26METBZOH & $88.7(6.5)$ & $86.7(4.7)$ & $90.7(4.5)$ & $101(6.7)$ & $99.3(4.1)$ & $88.7(9.1)$ & $93.3(6.1)$ & $94.7(7.0)$ \\
\hline
\end{tabular}

Table 4

Recoveries (\%) and RSD (\%) of the DI-HS-SPME-GC-MS/MS method in 8 real oilfield production waters spiked at $2.5 \mu \mathrm{g} \mathrm{L}^{-1}$.

\begin{tabular}{|c|c|c|c|c|c|c|c|c|}
\hline \multirow[t]{2}{*}{ Tracers } & \multicolumn{8}{|c|}{ \%Recovery (\%RSD) - samples spiked at $2.5 \mu \mathrm{g} \mathrm{L}-1$} \\
\hline & Snorre A & Snorre B & Ekofisk M & Gullfaks C & Heidrun A & Eldfisk A & Eldfisk S & Vigdis B \\
\hline PYR & $96.0(5.1)$ & $98.9(4.5)$ & $107(4.3)$ & $98.8(6.6)$ & $104(5.4)$ & $108(4.0)$ & $97.9(5.5)$ & $96.5(6.5)$ \\
\hline 23MPRZ & $99.7(6.4)$ & $98.3(5.2)$ & $100(4.1)$ & $104(9.2)$ & $94.0(7.0)$ & $95.6(4.8)$ & $97.5(6.3)$ & $106(4.8)$ \\
\hline $4 \mathrm{BZOH}$ & $92.8(3.4)$ & $105(7.4)$ & $104(4.3)$ & $92.0(6.1)$ & $92.5(6.0)$ & $103(3.7)$ & $94.0(6.5)$ & $99.5(6.8)$ \\
\hline 26BZOH & $101(5.4)$ & $91.7(5.8)$ & $94.0(2.8)$ & $92.8(7.4)$ & $105(7.3)$ & $95.5(8.4)$ & $92.0(6.9)$ & $101(7.1)$ \\
\hline 4METBZOH & $103(5.2)$ & $95.2(3.6)$ & $93.6(6.3)$ & $97.3(5.7)$ & $91.3(6.4)$ & $95.9(11)$ & $102(8.4)$ & $96.1(5.7)$ \\
\hline 26METBZOH & $91.3(6.3)$ & $93.1(4.0)$ & $94.8(6.9)$ & $91.0(8.2)$ & $96.0(6.2)$ & $96.4(4.7)$ & $96,5(3.7)$ & $94.4(6.4)$ \\
\hline
\end{tabular}

Mixed standard solutions were prepared in deionized water with concentrations starting at the calculated LOQ values and increasing, covering a wide range of values, to build calibration curves and evaluate the linearity of the method (the specific ranges for each compound are presented in Table 2). A direct linear proportional relationship was observed between the chromatographic response and the concentration of each of the analytes. Values for the coefficient of determination $\left(\mathrm{R}^{2}\right)$ were satisfactory and indicate good linear regression models for the chromatographic response vs concentration of each of the target compounds.

The precision of the full method was evaluated within a day (intra-day precision) and between 5 days (inter-day precision) at three different concentration levels $(0.5,2.5$, and $10 \mu \mathrm{g} / \mathrm{L})$. Results of the intra-day precision $(n=7)$ and inter-day precision $(n=5)$ are also summarized in Table 2.

The analytic method was used to screen for the 6 tracer compounds in production waters from 8 different Norwegian continental shelf oilfields (Snorre A, Snorre B, Ekofisk M, Gullfaks C, Heidrun A, Eldfisk A, Eldfisk S and Vigdis B). No signal of the presence of any of the analytes was detected in these samples. This is in agreement with one of the requirements for the technology: a tracer compound introduced into a given system, should be absent from it or present with a low and constant background so that the accuracy of the tracer test is not compromised. Of the 6 tracer compounds presented in the present manuscript only pyridine has been reported as a component of crude oils [41], however in relatively small amounts and mostly in the lighter hydrocarbon fractions. A PITT is primarily conceived for mature oilfields, thus the presence of pyridine (in significant amounts) is unlikely and the results from the present study back this up. The other 5 compounds have never, to the best of our knowledge, been described as part of any oilfield fluid, and their industrial and household use makes their presence highly unlikely [3].

Recovery studies of the 6 tracer compounds were then performed on the 8 production water samples at three different concentration levels. Because none of the target compounds was detected in the original samples, no correction to calculate the recoveries was required. Different linear ranges were obtained for the different compounds and so the values for the concentrations of the spikes were selected in an attempt to represent low, medium and high values for all analytes. The three levels of concentration were as follows: $0.50,2.50$ and $10 \mu \mathrm{g} \mathrm{L}^{-1}$, and the results for the recovery and \%RSD are presented in Tables 3-5.

The results show a good performance of the method developed with all the recovery values between $85 \%$ - 115\% and RSDs systematically $\leq 13 \%$. The lowest systematic recoveries were observed for the methoxybenzyl alcohols. This is mostly likely due to their low volatility combined with the fact that the dominant period of SPME extraction is performed on HS mode relatively to the time of DI mode ( 15 vs 5 minutes). Of all the tested compounds, the measured responses for $4 \mathrm{METBZOH}$ and $34 \mathrm{METBZOH}$ showed the largest difference relatively to HS-SPME (see Fig. 2) after 30 minutes of extraction. Such suggests that, although 5 minutes of DI enhances the analytical system's response, this is not enough time for as fast equilibrium to be reached in the adsorption system (water $\rightarrow$ headspace $\rightarrow$ SPME fiber) for 4METBZOH and 34METBZOH, as this is not an exhaustive extraction technique. However, in summary, the results show that the method is suitable for analysis of 
Table 5

Recoveries (\%) and RSD (\%) of the DI-HS-SPME-GC-MS/MS method in 8 real oilfield production waters spiked at $10 \mu \mathrm{L} \mathrm{L}^{-1}$.

\begin{tabular}{|c|c|c|c|c|c|c|c|c|}
\hline \multirow[t]{2}{*}{ Tracers } & \multicolumn{8}{|c|}{ \%Recovery (\%RSD) - samples spiked at $10 \mu \mathrm{g} \mathrm{L}^{-1}$} \\
\hline & Snorre A & Snorre B & Ekofisk M & Gullfaks C & Heidrun A & Eldfisk A & Eldfisk S & Vigdis B \\
\hline PYR & $96.0(4.5)$ & $97.8(4.6)$ & $96.4(3.9)$ & $97.4(4.1)$ & $101(4.0)$ & $102(4.1)$ & $101(2.7)$ & $96.7(4.5)$ \\
\hline 23MPRZ & $95.6(1.6)$ & $99.3(4.5)$ & $98.8(3.3)$ & $97.5(5.0)$ & $97.6(4.2)$ & $102(4.2)$ & $103(5.8)$ & $95.8(4.4)$ \\
\hline $4 \mathrm{BZOH}$ & $102(4.2)$ & $102(3.8)$ & $101(2.8)$ & $95.2(3.5)$ & $94.3(5.6)$ & $97.0(3.5)$ & $95.8(4.9)$ & $95.9(6.2)$ \\
\hline $26 \mathrm{BZOH}$ & $97.8(3.1)$ & $102(5.2)$ & $97.9(3.7)$ & $98.9(4.3)$ & $97.6(3.5)$ & $102(4.8)$ & $96.6(4.6)$ & $96.1(4.9)$ \\
\hline 4METBZOH & $96.2(3.9)$ & $94.7(5.7)$ & $97.8(3.2)$ & $95.8(4.7)$ & $95.7(4.9)$ & $94.6(4.8)$ & $93.2(3.8)$ & $95.9(5.7)$ \\
\hline 34METBZOH & $95.8(5.2)$ & $92.2(5.9)$ & $95.5(4.0)$ & $98.9(3.6)$ & $96.0(4.4)$ & $90.7(4.2)$ & 93.7 (3.9) & $95.7(5.0)$ \\
\hline
\end{tabular}

the 6 tracers in the intend matrix with a high sample output capacity.

\section{Conclusions}

An easy to automate analytical method consisting of sequential DI-HS-SPME extraction coupled to gas-phase chromatography and tandem mass spectrometry (GC-MS/MS) was developed for the identification and quantification of 4-chlorobenzyl alcohol, 2,6-dichlorobenzyl alcohol, 4-methoxybenzyl alcohol, 3,4dimethoxybenzyl alcohol, pyridine, and 2,3-dimethylpyrazine in production waters from oilfields. These compounds are promising PITT tracer candidates and a real test based on their use implies the analyses of hundreds of samples during a tracer campaign.

A DI-SPME approach combined with the addition of $\mathrm{NaCl}$ produced the best results of extraction, however proved unsuitable for real samples due to matrix effects. Sequential DI-HS-SPME was adopted to overcome this drawback and temperature and time of extraction were optimized. The final SPME extraction procedure consists of $5 \mathrm{~mL}$ of sample at pH 9.0 with $1.8 \mathrm{~g}$ of $\mathrm{NaCl}$, constant stirring, 5 minutes of DI-SPME followed by 15 minutes of HS-SPME at $70{ }^{\circ} \mathrm{C}$ using a DVB/CAR/PDMS $(50 / 30 \mu \mathrm{m})$ fiber.

The linearity and precision of the method were validated for all 6 target analytes. Linear behavior was observed for a wide range of concentrations (medium-low ng $\mathrm{L}^{-1}$ to low $\mu \mathrm{g} \mathrm{L}^{-1}$ ) and the LOQs were calculated to be between 0.080 and $0.35 \mu \mathrm{g} \mathrm{L}^{-1}$. The method's recovery was evaluated at 3 concentration levels $(0.50$, 2.5 and $10 \mu \mathrm{g} \mathrm{L}^{-1}$ ) in 8 real production waters from Norwegian offshore oilfields. The obtained recovery values were systematically higher than $85 \%$ and RSDs lower than $13 \%$.

The sequential DI-HS-SPME-GC-MS/MS method was used to screen the production waters in the present study for the presence of the 6 compounds of interest. None of these compounds was detected in any of the samples, fact in line with the requirements for their use as an oilfield tracer.

\section{Declaration of Competing Interest}

The authors declare that they have no known competing financial interests or personal relationships that could have appeared to influence the work reported in this paper.

\section{CRediT authorship contribution statement}

Mario Silva: Conceptualization, Methodology, Formal analysis, Investigation, Writing - original draft, Visualization, Validation, Writing - review \& editing. Tor Bjørnstad: Validation, Writing review \& editing, Resources, Supervision, Project administration, Funding acquisition.

\section{Acknowledgements}

The authors acknowledge the Research Council of Norway and the industry partners, ConocoPhillips Skandinavia AS, Aker BP ASA,
Eni Norge AS, Maersk Oil, a company by Total, Statoil Petroleum AS, Neptune Energy Norge AS, Lundin Norway AS, Halliburton AS, Schlumberger Norge AS, Wintershall Norge AS, and DEA Norge AS, of The National IOR Centre of Norway for support.

\section{Supplementary materials}

Supplementary material associated with this article can be found, in the online version, at doi:10.1016/j.chroma.2020.461508.

\section{References}

[1] C. Carpenter, Use of Partitioning Tracers to Estimate Oil-Saturation Distribution, SPE-0916-0072-JPT, 68 (2016) 72-73.

[2] J.S. Tang, Partitioning Tracers and In-Situ Fluid Saturation Measurements, SPE22344-PA, 10 (1995) 33-39.

[3] M. Silva, H. Stray, T. Bjørnstad, Stability assessment of PITT tracer candidate compounds: the case of benzyl alcohols, J. Petroleum Sci. Eng. 167 (2018) 517-523.

[4] A. Muggeridge, A. Cockin, K. Webb, H. Frampton, I. Collins, T. Moulds, P. Salino, Recovery rates, enhanced oil recovery and technological limits, Philos. Transact. A Math. Phys. Eng. Sci. 372 (2014) 20120320.

[5] IEA, Key World Energy Statistics 2016, IEA, Paris, 2016.

[6] M. Sanni, M. Al-Abbad, S. Kokal, O. Dugstad, S. Hartvig, O. Huseby, Pushing the envelope of residual oil measurement: a field case study of a new class of inter-well chemical tracers, J. Petroleum Sci. Eng. 163 (2018) 538-545.

[7] C.E.J. Cooke, Method of Determining Fluid Saturations in Reservoirs, Esso Production Research Company, USA, 1971 (US Patent 3,590,923).

[8] S.O. Viig H. Juilla, P. Renouf, R. Kleven, B. Krognes, O. Dugstad, O.K. Huseby, Application of a new class of chemical tracers to measure oil saturation in partitioning interwell tracer tests, SPE International Symposium on Oilfield Chemistry, Society of Petroleum Engineers, 2013.

[9] M.A. AlAbbad, M.L. Sanni, S. Kokal, A. Krivokapic, C. Dye, Ø. Dugstad, S.K. Hartvig, O.K. Huseby, A Step Change for Single-Well Chemical-Tracer Tests: Field Pilot Testing of New Sets of Novel Tracers, SPE-181408-PA, Preprint (2018) 13.

[10] C. Serres-Piole, H. Preud'homme, N. Moradi-Tehrani, C. Allanic, H. Jullia, R. Lobinski, Water tracers in oilfield applications: guidelines, J. Petroleum Sci. Eng. 98-99 (2012) 22-39.

[11] M. Silva, H. Stray, T. Bjørnstad, Stability assessment of PITT tracer candidate compounds - the case of pyrazines, J. Petroleum Sci. Eng. 182 (2019) 106269.

[12] M. Silva, H. Stray, T. Bjørnstad, Studies on new chemical tracers for determination of residual oil saturation in the inter-well region, SPE Oklahoma City Oil and Gas Symposium, Society of Petroleum Engineers, Oklahoma City, Oklahoma, USA, 2017.

[13] K. Muller, A. Seubert, Ultra trace determination of fluorobenzoic acids in tap and reservoir water using solid-phase extraction and gas chromatographymass spectrometry, J. Chromatogr. A 1260 (2012) 9-15.

[14] K. Muller, A. Seubert, Ultra trace determination of fluorobenzoic acids in reservoir and ground water using isotope dilution gas chromatography mass spectrometry, Isot. Environ. Health Stud. 50 (2014) 277-284

[15] H. Preudhomme, C.S. Piole, A. Commarieu, F. Aubertin, Method for detecting tracer compounds for hydrocarbon production (US patent 8853619B2), in, Total SA USA, 2014

[16] E. Boyacı, Á. Rodríguez-Lafuente, K. Gorynski, F. Mirnaghi, É.A. Souza-Silva, D. Hein, J. Pawliszyn, Sample preparation with solid phase microextraction and exhaustive extraction approaches: comparison for challenging cases, Anal. Chim. Acta 873 (2015) 14-30.

[17] E. Boyaci, K. Gorynski, C.R. Viteri, J. Pawliszyn, A study of thin film solid phase microextraction methods for analysis of fluorinated benzoic acids in seawater J. Chromatogr. A 1436 (2016) 51-58.

[18] É.A. Souza-Silva, R. Jiang, A. Rodríguez-Lafuente, E. Gionfriddo, J. Pawliszyn, A critical review of the state of the art of solid-phase microextraction of complex matrices I. Environmental analysis, TrAC Trends Anal. Chem. 71 (2015) 224-235.

[19] É.A. Souza-Silva, E. Gionfriddo, J. Pawliszyn, A critical review of the state of the art of solid-phase microextraction of complex matrices II. Food analysis, TrAC Trends Anal. Chem. 71 (2015) 236-248. 
[20] É.A. Souza-Silva, N. Reyes-Garcés, G.A. Gómez-Ríos, E. Boyacı, B. Bojko, J. Pawliszyn, A critical review of the state of the art of solid-phase microextraction of complex matrices III. Bioanalytical and clinical applications, TrAC Trends Anal. Chem. 71 (2015) 249-264.

[21] J.S. Câmara, M.A. Alves, J.C. Marques, Multivariate analysis for the classification and differentiation of Madeira wines according to main grape varieties, Talanta 68 (2006) 1512-1521.

[22] E.G. Dennis, R.A. Keyzers, C.M. Kalua, S.M. Maffei, E.L. Nicholson, P.K. Boss, Grape contribution to wine aroma: production of hexyl acetate, octyl acetate, and benzyl acetate during yeast fermentation is dependent upon precursors in the must, J. Agric. Food Chem. 60 (2012) 2638-2646.

[23] P. Porto-Figueira, J.A. Figueira, P. Berenguer, J.S. Câmara, Exploring a volatomic-based strategy for a fingerprinting approach of Vaccinium padifolium L. berries at different ripening stages, Food Chem. 245 (2018) 141-149.

[24] R.P. Goswami, G.K. Jayaprakasha, K. Shetty, B.S. Patil, Lactobacillus plantarum and natural fermentation-mediated biotransformation of flavor and aromatic compounds in horse gram sprouts, Process Biochem. 66 (2018) 7-18.

[25] R.F.J. da Rocha, Í.M. da Silva Araújo, S.M. de Freitas, D. dos Santos Garruti, Optimization of headspace solid phase micro-extraction of volatile compounds from papaya fruit assisted by GC-olfactometry, J. Food Sci. Technol. 54 (2017) 4042-4050.

[26] P. Kusch, G. Knupp, Analysis of residual styrene monomer and other volatile organic compounds in expanded polystyrene by headspace solid-phase microextraction followed by gas chromatography and gas chromatography/mass spectrometry, J. Sep. Sci. 25 (2002) 539-542.

[27] G. Pieraccini, S. Furlanetto, S. Orlandini, G. Bartolucci, I. Giannini, S. Pinzauti, G. Moneti, Identification and determination of mainstream and sidestream smoke components in different brands and types of cigarettes by means of solid-phase microextraction-gas chromatography-mass spectrometry, J. Chromatogr. A 1180 (2008) 138-150.

[28] M. Gröning, M. Hakkarainen, Headspace solid-phase microextraction with gas chromatography/mass spectrometry reveals a correlation between the degradation product pattern and changes in the mechanical properties during the thermooxidation of in-plant recycled polyamide 6,6, J. Appl. Polym. Sci. 86 (2002) 3396-3407.

[29] S. Legrand, J. Dugay, J. Vial, Use of solid-phase microextraction coupled with gas chromatography for the determination of residual solvents in pharmaceutical products, J. Chromatogr. A 999 (2003) 195-201.
[30] S. Rocha, L. Maeztu, A. Barros, C. Cid, M.A. Coimbra, Screening and distinction of coffee brews based on headspace solid phase microextraction/gas chromatography/principal component analysis, J. Sci. Food Agric. 84 (2004) 43-51.

[31] H.M. Bettenhausen, L. Barr, C.D. Broeckling, J.M. Chaparro, C. Holbrook, D. Sedin, A.L. Heuberger, Influence of malt source on beer chemistry, flavor, and flavor stability, Food Res. Int. 113 (2018) 487-504.

[32] N.V. Bakaikina, B. Kenessov, N.V. Ul'yanovskii, D.S. Kosyakov, Quantification of transformation products of rocket fuel unsymmetrical dimethylhydrazine in soils using SPME and GC-MS, Talanta 184 (2018) 332-337.

[33] D.C. Frank, C.M. Owen, J. Patterson, Solid phase microextraction (SPME) combined with gas-chromatography and olfactometry-mass spectrometry for characterization of cheese aroma compounds, LWT - Food Sci. Technol. 37 (2004) 139-154.

[34] G. Liu, S. Xu, X. Wang, Q. Jin, X. Xu, Y. Shen, G. Xu, H. Zhang, Analysis of the volatile components of tea seed oil (Camellia sinensis O. Ktze) from China using HS-SPME-GC/MS, Int. J. Food Sci. Technol. 51 (2016) 2591-2602.

[35] C. Sala, M. Mestres, M.P. Martı́, O. Busto, J. Guasch, Headspace solid-phase microextraction analysis of 3-alkyl-2-methoxypyrazines in wines, J. Chromatogr. A 953 (2002) 1-6.

[36] S.-Y. Moon, M.A. Cliff, E.C.Y. Li-Chan, Odour-active components of simulated beef flavour analysed by solid phase microextraction and gas chromatography-mass spectrometry and -olfactometry, Food Res. Int. 39 (2006) 294-308.

[37] P. Makoś, A. Przyjazny, G. Boczkaj, Methods of assaying volatile oxygenated organic compounds in effluent samples by gas chromatography-a review, J. Chromatogr. A 1592 (2019) 143-160.

[38] A. Fakhru'l-Razi, A. Pendashteh, L.C. Abdullah, D.R.A. Biak, S.S. Madaeni, Z.Z. Abidin, Review of technologies for oil and gas produced water treatment, J. Hazard. Mater. 170 (2009) 530-551.

[39] P. Makoś, A. Fernandes, G. Boczkaj, Method for the determination of carboxylic acids in industrial effluents using dispersive liquid-liquid microextraction with injection port derivatization gas chromatography-mass spectrometry, J. Chromatogr. A 1517 (2017) 26-34.

[40] Eurachem, The Fitness for Purpose of Analytical Methods: a Laboratory Guide to Method Validation and Related Topics, second ed., 2014 www.eurachem.org.

[41] G.H.C. Prado, Y. Rao, A. de Klerk, Nitrogen removal from oil: a review, Energy Fuels 31 (2017) 14-36. 



\section{Paper VII}

Variation of the partition coefficient of phase-partitioning compounds between hydrocarbon and aqueous phases: an experimental study.

Mário Silva, Helge Stray, Mahmoud Ould Metidji, Tor Bjørnstad (Submitted 2020). Fuel, (under review). 
1 Variation of the partition coefficient of phase-partitioning compounds

2 between hydrocarbon and aqueous phases: an experimental study:

4 Mario Silva ${ }^{1,2,3, a}$; Helge Stray ${ }^{3}$ Mahmoud Ould Metidji1,3; Tor Bjørnstad ${ }^{1,3}$

$5{ }^{1}$ The National IOR Centre of Norway, University of Stavanger, 4036 Stavanger, Norway

$6 \quad{ }^{2}$ Department of Energy Resources, University of Stavanger, 4036 Stavanger, Norway

$7{ }^{3}$ Institute for Energy Technology (IFE), Department of Tracer Technology, Instituttveien 18,

$8 \quad 2007$ Kjeller, Norway

$9 \quad{ }^{a}$ Corresponding author: mario.silva@ife.no; mariohsilva@sapo.pt 
Many non-ionic chemical compounds will form real solutions in equilibrium between two immiscible phases in contact. The partition coefficient $(\mathrm{K})$ is defined as the quotient between the equilibrium concentration of the substance in the hydrocarbon and the aqueous phase. The reversible partition of a compound between hydrocarbon and aqueous phases is the basis of the partitioning inter-well tracer test (PITT). PITTs are of high interest for the characterization of oil reservoirs, in hydrogeology for assessment of the contamination of soils by non-aqueous phase liquids (NAPLs), and in process technology. The $\mathrm{K}$ value of substance is influenced by the actual chemical and physical conditions of the system where it will be used as phase-partitioning tracer. Thus, it is important to evaluate the extent of variation that the $\mathrm{K}$ value can exhibit under different relevant conditions. In the present document, we report the methodology and findings from the experimental determination of the $\mathrm{K}$ values of 4-methoxybenzyl alcohol, 3,4-dimethoxybenzyl alcohol, 4-chlorobenzyl alcohol, 2,6dichlorobenzyl alcohol, pyridine, 2,3-dimethylpyrazine and 2,6-dimethylpyrazine between different hydrocarbon and aqueous phases. These 7 compounds were previously identified as interesting PITT tracer candidates. Individual K-values were determined under different temperatures, compositions of the hydrocarbon phase (synthetic mixtures of toluene, isooctane, and 1-octanol and 5 real crude oils), and different ionic strengths (I) and ionic compositions of the aqueous phase. The reversibility of the partitioning phenomena was also evaluated. The composition of the hydrocarbon phase and the ionic strength of the water phase were found to influence the $\mathrm{K}$ values of all seven compounds. Results suggest that the substitution of monovalent ions with divalent ions in the aqueous phase, keeping the ionic strength constant, does not influence the $\mathrm{K}$ values. Temperature effects on the $\mathrm{K}$-values are always visible for 3,4-dimethoxybenzyl alcohol, 2,6-dichlorobenzyl alcohol, pyridine, 2,3dimethylpyrazine and 2,6-dimethylpyrazine. Dependent on the hydrocarbon phase composition, temperature also influences the K-values for 4-methoxybenzyl alcohol and 4-chlorobenzyl alcohol.

Keywords: PITT, Phase-partitioning, Tracers, IOR, lonic strength, Hydrocarbon, Temperature 
$50 \quad S_{O R}=\frac{T_{R}-T_{W}}{T_{R}+T_{W}(K-1)}$

51 Where $T_{R}$ is the retention time of the partitioning tracer, $T_{W}$ the retention time of the passive water 52 tracer, and $\mathrm{K}$ the oil/water partition coefficient of the partitioning tracer. Eq. 1 is the result of a mass

$57 \quad K=\frac{C_{T O}}{C_{T W}}$

\section{Introduction}

Further exploration of conventional mature water-flooded oil reservoirs is necessary to satisfy the global hydrocarbon demand within the "stated policies" scenario [1]. Thus, improved oil recovery (IOR) projects will take a central part in oil production. These projects are often expensive and require a thorough knowledge about the oil reservoir. One of the most important parameters is the knowledge of the residual oil saturation ( $\mathrm{S}_{\mathrm{OR}}$ ) in the flooded volumes between injector/producer well pairs. SoR can be used to identify IOR targets, select IOR methods, and assess IOR operations. Knowledge of SoR can be obtained through the partitioning inter-well tracer test (PITT). The PITT measures SoR (or even remaining oil saturation) in the inter-well region of water-flooded oil reservoirs and is based on the production delay of an oil/water partitioning tracer relatively to a passive water tracer [2]. This delay is caused by the equilibrium distribution of the partitioning tracer between the near-stagnant hydrocarbon phase and the flowing aqueous phase. Sor can be obtained from the time of arrival of the tracers on the producer well (either by direct calculation or incorporation in a reservoir simulator) as follows [3, 4]:

balance of the transport of the tracers through the porous medium of an oil reservoir, assuming a stagnant hydrocarbon phase and an instantaneous equilibrium distribution of the partitioning tracer between the oil phase and the water phase [5]. $\mathrm{K}$ is defined as the quotient between the equilibrium concentration of the tracer in the hydrocarbon-phase and the aqueous-phase:

Where $\left(\mathrm{C}_{\mathrm{TO}}\right)$ is the equilibrium concentration of the tracer in the oil/hydrocarbon phase and $\left(\mathrm{C}_{\mathrm{TW}}\right)$ its equilibrium concentration in the aqueous phase, when both phases are in contact. 
While $T_{R}$ and $T_{W}$ in Eq. 1 are experimental data resulting from the field test, the determination of the $K$ value of a compound is typically performed in laboratory experiments that take into consideration the chemical and physical conditions of the involved organic and aqueous fluids [3, 6-9]. Such experiments are "static", based on the produced fluids, and do not account for the variations that can occur in the field in the flooded regions between injector/producer well-pairs, such as varying temperature, salinity, or composition of the hydrocarbon phase in the path between injector/producer pairs. The partition of a substance between a hydrocarbon phase and an aqueous phase has been shown to be influenced by these parameters [10-12]. For a given system, with fixed conditions, the relationship between the equilibrium concentration of a substance soluble in both of the two immiscible phases in contact is a constant [13]. This means that, under fixed system conditions, $\mathrm{K}$ is independent from the initial concentration of the substance in any of the phases. Once the phases become in contact, the concentrations of the substance in both phases will vary until $K$ reaches the value for the conditions of the system. Temperature, composition of the hydrocarbon-phase and concentration of salts in the water cause variation of the K-values, and absence of mutual phase saturation before contact of the phases and uncontrolled experimental conditions leading to results reported before distribution equilibrium is achieved, cause erroneously measured values of $\mathrm{K}$ for the actual experimental conditions [14-17]. To ensure accuracy of SoR measurements, it is therefore important to understand how, and on what magnitude, variations in relevant factors of a hydrocarbon/water system affect the $K$ value of substances used as phase-partitioning tracers.

The present work is primarily experimental in nature and intended to evaluate the effects of temperature, hydrocarbon composition, salt content of the aqueous phase and nature of the ions, and $\mathrm{pH}$ on the $\mathrm{K}$ value of phase-partitioning compounds. The results obtained can be used to correct the values of $\mathrm{K}$ used in Eq. 1 for $\mathrm{S}_{\mathrm{OR}}$ determination based on the data of the field tests. For this purpose, seven molecules (2,3-dimethylpyrazine; 2,6-dimethylpyrazine; pyridine; 4-chlorobenzyl alcohol; 2,6dichlorobenzyl alcohol; 4-methoxybenzyl alcohol and 3,4-dimethoxybenzyl alcohol) previously identified as promising phase-partitioning tracer candidates [18] were used as partitioning tracers. The 
86 experiments performed also evaluated the occurrence of partitioning and its reversibility, to ensure that

87 a surfactant like behaviour was not observed. The $\mathrm{K}$ values were determined for systems with a 88 synthetic hydrocarbon mixture as well as with real crude oil, using five different oils from fields in 89 operation on the Norwegian continental shelf.

\section{2. Materials and Methods}

91

92

93

94

95

96

97 the respective operators.

98 The acronyms for the compounds used in the present study and some of their relevant properties are 99 presented in Table 1.

100

\begin{tabular}{|c|c|c|c|c|c|}
\hline Compound & $\begin{array}{c}\text { M.W. } \\
\left(\mathrm{g} \mathrm{mol}^{-1}\right)\end{array}$ & $\log P$ & M.P. $\left({ }^{\circ} \mathrm{C}\right)$ & B.P. $\left({ }^{\circ} \mathrm{C}\right)$ & $\mathrm{pKa}$ \\
\hline Pyridine & 79,04 & 0,65 & -42 & 115 & $5,25^{*}$ \\
\hline 2,3-Dimethylpyrazine & 108,07 & 0,54 & -12 & 156 & $1,62^{*}$ \\
\hline 2,6-Dimethylpyrazine & 108,07 & 0,55 & 37 & 154 & $1,55^{*}$ \\
\hline 4-Chlorobenzyl alcohol & 142,02 & 0,82 & 71 & 232 & 13,9 \\
\hline 2,6-Dichlorobenzyl alcohol & 175,98 & 1,07 & 98 & 268 & 13,5 \\
\hline 4-Methoxybenzyl alcohol & 138,07 & 0,71 & 23 & 259 & 13,6 \\
\hline 3,4-Dimethoxybenzyl alcohol & 168,08 & 0,62 & 22 & 297 & 13,3 \\
\hline \multicolumn{6}{|l|}{ Data Source: NIST chemistry WebBook } \\
\hline
\end{tabular}

Table 1. Some relevant physico-chemical properties and acronyms of the studied compounds 


\subsection{Analytical instrumentation}

The present study used two different techniques for identification and quantification of the studied compounds. Ultra-performance liquid chromatography (UPLC) coupled with UV detection was primarily used to quantify the tracer compounds in aqueous phases. An UPLC Agilent Technologies 1290 Infinity II (Agilent Technologies, Santa Clara, California, USA) equipped with a Waters "Acquity UPLC BEH" reversed-phase C18 $1.7 \mu \mathrm{m}$ packed column (Waters Corporation, Milford, Massachusetts, USA) was employed. The benzyl alcohols were detected at $222 \mathrm{~nm}$, while the pyrazines and pyridine were detected at $254 \mathrm{~nm}$. Gradient elution was used for efficient separation of the target compounds. The flow of the mobile phase was $0,5 \mathrm{~mL} / \mathrm{min}$ and it was composed of 3 eluents: $5 \mathrm{mM} \mathrm{NH}_{4} \mathrm{HCO}_{2}(\mathrm{~A})$, methanol $(B)$ and acetonitrile $(C)$. The initial composition of the mobile phase consisted of $98 \% A, 1 \% B$ and $1 \% \mathrm{C}$. The fraction of eluent $\mathrm{B}$ increased to $20 \%$ after 4 minutes and remained at this value for 1,5 minutes. $\mathrm{C}$ was kept constant at $1 \%$. The fraction of $\mathrm{B}$ was then increased to $70 \%$ on minute 8 and kept for 0,5 minute. The fraction of $\mathrm{C}$ was again kept constant at $1 \%$. The mobile phase conditions were restored to the initial ones at minute 9 and maintained for 1 minute. The total time of the chromatographic run was 10 minutes.

Gas-phase chromatography (GC) coupled with mass spectrometry (MS) was used primarily to identify and quantify the compounds in the organic phase. A Thermo Scientific Trace ${ }^{\mathrm{TM}} 1310$ gas chromatograph (Thermo Fischer Scientific, Waltham, MA, USA) equipped with a chromatographic column Restek Rtx®-5MS (30 m X $0.25 \mathrm{~mm}$ X $0.25 \mu \mathrm{m}$ ) and coupled with a triple quadrupole mass spectrometer Thermo Scientific TSQ 8000 (Thermo Fischer Scientific, Waltham, MA, USA) was employed. $1 \mu \mathrm{L}$ of sample with appropriate dilution was injected in split mode (1:20). The temperature of the injector was $250{ }^{\circ} \mathrm{C}$. The carrier gas consisted of helium (99.999\%) at $1 \mathrm{~mL} / \mathrm{min}$. The initial temperature of the oven was $50^{\circ} \mathrm{C}$ and the value was maintained for 3 minutes. This was followed by a heating ramp of $30^{\circ} \mathrm{C} / \mathrm{min}$ to $290^{\circ} \mathrm{C}$. Temperature was kept at $290{ }^{\circ} \mathrm{C}$ for 8 minutes. The temperature 
126 of the ion transfer line was $290{ }^{\circ} \mathrm{C}$ and the ion source was kept at $320^{\circ} \mathrm{C}$. The MS was operated in 127 electron impact $(\mathrm{EI})$ ionization mode $(+70 \mathrm{eV})$.

\subsection{Experimental procedure}

The details of the experimental procedures to study the influence of each of the tested variables on the

$\mathrm{K}$ value of studied compounds are presented hereafter. All experiments were based on the so called "shake flask method". This method consists of the mixing of two immiscible phases with the solute candidate tracer dissolved in one of them. After equilibrium has been reached, the concentrations of the solutes were measured in one or both phases. In all experiments the hydrocarbon phase and the aqueous phase to be used in the binary system were mutually saturated with each other before partition experiments with the solutes commenced. Only after this process the compounds under study were added (typically to the aqueous phase) by dilution of aliquots from a mother solution of known concentration. This step consisted of adding small volumes (between $500 \mu \mathrm{L}-1 \mathrm{~mL}$ ) of the concentrated solution of the tracers to $500 \mathrm{~mL}$ of the aqueous phase previously saturated with the organic phase.

\subsubsection{Preliminary partition experiments and influence of $\mathrm{pH}$}

141 Two aqueous solutions were prepared containing the seven studied compounds, each at a concentration of $10 \mathrm{mg} \mathrm{L}^{-1}$, with salinities composed of 2,5\% $\mathrm{NaCl}$ and 5,0\% $\mathrm{NaCl}(\mathrm{m} / \mathrm{V})$, respectively. 5 aliquots were taken of each of the solutions and their $\mathrm{pH}$ was adjusted to 5, 6, 7, 8 and 9, respectively, by adding either $\mathrm{HCl}$ or $\mathrm{NaOH}$. A "model oil" synthetic hydrocarbon solution composed of $20 \%$ (vol.) toluene, $70 \%$ (vol.) iso-octane and $10 \%$ (vol.) octanol was prepared. Thus, a mixture of hydrocarbons composed of branched alkanes, aromatic, and aliphatic slightly polar components was obtained. The mixture was used in further experiments with varying fractions of iso-octane and 1-octanol to evaluate the effects of varying aliphatic composition and polarity of the hydrocarbons on the partitioning of the investigated molecules. An aliquot of $15 \mathrm{~mL}$ was taken from each of the aqueous solutions at the different $\mathrm{pH}$ values containing the studied compounds and placed in $20 \mathrm{~mL}$ vials with aluminium caps 
with PTFE septum. $5 \mathrm{~mL}$ of the model oil were added to each of the vials. The mixture was thoroughly shaken and placed in a thermal cabinet at $25^{\circ} \mathrm{C}$ for $24 \mathrm{~h}$. After $24 \mathrm{~h}$, a $2 \mathrm{~mL}$ sample was taken from the aqueous phase of each vial using a syringe with a 24 ga needle. The concentration of the tracer compounds was then determined in these samples. All experiments were performed in triplicate. Using a mass balance, the concentration of the compounds in the hydrocarbon phase can be determined:

$$
C_{T O}=\frac{\left(C_{T W i}-C_{T W f}\right) \cdot V_{W}}{V_{O}}
$$

157 Where $\mathrm{C}_{\mathrm{To}}$ is the concentration of the tracers in the hydrocarbon phase, $\mathrm{C}_{\mathrm{TW}}$ is the concentration of the tracers in the aqueous phase before contact with the hydrocarbon phase, $\mathrm{C}_{\mathrm{TWf}}$ the concentration of the tracers in the aqueous phase after the equilibrium contact with the hydrocarbon phase, $V_{W}$ is the volume of aqueous solution and $\mathrm{V}_{\mathrm{O}}$ the volume of the model oil used in the experiments.

2.3.2. Influence of hydrocarbon composition, water salinity and temperature on the $\mathrm{K}$ values

Solutions of the potential tracer compounds under study were prepared at a concentration of $10 \mathrm{mg} \mathrm{L}^{-1}$ in deionized water, $2,5 \% \mathrm{NaCl}, 5,0 \% \mathrm{NaCl}, 7,5 \% \mathrm{NaCl}$ and $10 \% \mathrm{NaCl}$. The $\mathrm{pH}$ of these solutions was adjusted to $8,0 \pm 0,1$ by adding $280 \mathrm{mg} \mathrm{L}^{-1}$ of $\mathrm{NaHCO}_{3}$. A model oil composed of $20 \%$ toluene (vol.) and varying fractions of octanol $(0 \%-20 \%$ vol.) and iso-octane $(60 \%-80 \%$ vol) was used as the hydrocarbon phase. The purpose of using such mixtures was to evaluate the effect of polarity and 167 "nature" of the organic phase (more aromatic or more aliphatic) on the partitioning of the tested compounds. Series of $15 \mathrm{~mL}$ of each of the tracer solutions were placed in $20 \mathrm{~mL}$ vials with aluminium caps with PTFE septum together with $5 \mathrm{~mL}$ of the various model oil mixtures. These mixtures were shaken and placed in thermal cabinets at $25^{\circ} \mathrm{C}, 50^{\circ} \mathrm{C}, 75^{\circ} \mathrm{C}$ and $100^{\circ} \mathrm{C}$ respectively to evaluate the 171 effect of temperature on the partitioning. After $24 \mathrm{~h}$, samples of the aqueous phases were taken, and the 172 concentration of the tracers determined by UPLC-UV.

173 In order to verify the reversibility of the partitioning and its independence of initial concentration, series 174 of samples of $2 \mathrm{~mL}$ of the model oil solutions containing the chemical compounds from the experiments 
175 above were taken and transferred to $10 \mathrm{~mL}$ vials with aluminium caps with PTFE septum. $5 \mathrm{~mL}$ of the 176 previously prepared solutions of $2,5 \% \mathrm{NaCl}, 5,0 \% \mathrm{NaCl}, 7,5 \mathrm{NaCl}$ and $10 \% \mathrm{NaCl}$ respectively were 177 mixed with the $2 \mathrm{~mL}$ organic phase and the incubation process and analytical process repeated.

178 Many of the results obtained for 2 compounds (4-chlorobenzyl alcohol and 3,4-dimethoxybenzyl 179 alcohol) required their individual re-testing. $\mathrm{K}$ values for 4-chlorobenzyl alcohol exhibited sometimes 180 very high values and the opposite was observed for 3,4-dimethoxybenzyl alcohol. In both cases, an 181 accurate determination of the $\mathrm{K}$ value could be compromised if experimental adjustments were not 182 done, as variations in the concentrations of these compounds could not be accurately determined. The 183 same procedure described in the present point was employed with different initial concentrations of the 184 two compounds in solution. When required, the initial concentrations of 4-chlorobenzyl alcohol and 3,4185 dimethoxybenzyl alcohol were of $200 \mathrm{mg} \mathrm{L}^{-1}$ and $1 \mathrm{mg} \mathrm{L}^{-1}$, respectively.

186 2.3.3. Influence of the presence of divalent ions in the aqueous phase

187 To examine the effect of replacing monovalent ions with divalent ions at the same ionic strength, the 188 experiments performed as described in 2.3.2. were repeated using a fixed "model oil" mixture 189 composed of $80 \%(\mathrm{vol})$ iso-octane and $20 \%(\mathrm{vol})$ toluene. The aqueous solutions of the partitioning 190 compounds were prepared in a mixed solution of monovalent and divalent ions building up the same 191 ionic strength (I) as the ones using only $\mathrm{NaCl}$. Equation 5 was used to calculate I (expressed in M).

$192 \quad \mathrm{I}=\frac{1}{2} \sum_{i=1}^{n} C_{i} Z_{i}^{2}$

193 Where $C_{i}$ is the molar concentration of ion $i(M), Z_{i}$ is the charge number of that same ion and $n$ is the 194 number of different ions.

195 The amount of $\mathrm{NaCl}$ present in each solution was reduce to $1 / 4$ relatively to those described in chapter 196 2.3.2., and $\mathrm{Na}_{2} \mathrm{SO}_{4}, \mathrm{CaCl}_{2}$ and $\mathrm{MgCl}_{2}$ were added in sufficient amounts to reach the same values of $\mathrm{I}$. 197 Table 2 presents the amounts of each salt used and I of each solution. 
Table 2. Salt, respective amount present, and I of the aqueous solutions of the studied compounds.

\begin{tabular}{|c|c|c|c|c|c|c|c|c|}
\hline $\begin{array}{c}\text { C. } \mathrm{NaCl} \\
(\mathrm{g} / \mathrm{L})\end{array}$ & $\begin{array}{c}\text { C. } \mathrm{NaCl} \\
\text { (M) }\end{array}$ & $\begin{array}{c}\text { C. } \mathrm{Na}_{2} \mathrm{SO}_{4} \\
(\mathrm{~g} / \mathrm{L})\end{array}$ & $\begin{array}{c}\text { C. } \mathrm{Na}_{2} \mathrm{SO}_{4} \\
\text { (M) }\end{array}$ & $\begin{array}{c}\text { C. } \mathrm{CaCl}_{2} \\
(\mathrm{~g} / \mathrm{L})\end{array}$ & $\begin{array}{c}\text { C. } \mathrm{CaCl}_{2} \\
\text { (M) }\end{array}$ & $\begin{array}{c}\text { C. } \mathrm{MgCl}_{2} \\
(\mathrm{~g} / \mathrm{L})\end{array}$ & $\begin{array}{c}\text { C. } \mathrm{MgCl}_{2} \\
\text { (M) }\end{array}$ & $I(M)$ \\
\hline $25^{*}$ & 0,43 & 0 & 0 & 0 & 0 & 0 & 0 & 0,43 \\
\hline 50 * & 0,86 & 0 & 0 & 0 & 0 & 0 & 0 & 0,86 \\
\hline $75^{*}$ & 1,28 & 0 & 0 & 0 & 0 & 0 & 0 & 1,28 \\
\hline 100 * & 1,71 & 0 & 0 & 0 & 0 & 0 & 0 & 1,71 \\
\hline 6,25 & 0,11 & 2,0 & 0,014 & 5,1 & 0,046 & 4,4 & 0,046 & 0,43 \\
\hline 12,5 & 0,22 & 2,0 & 0,014 & 11,1 & 0,10 & 9,5 & 0,10 & 0,86 \\
\hline 18,75 & 0,32 & 2,0 & 0,014 & 17,0 & 0,15 & 14,6 & 0,15 & 1,28 \\
\hline 25 & 0,43 & 2,0 & 0,014 & 23,0 & 0,21 & 19,7 & 0,21 & 1,71 \\
\hline
\end{tabular}

${ }^{*}$ Experiments described in 2.3.2. are present in this table to show the value of I.

201 The procedure described in 2.3.2. was employed using the real (stock tank) oils in replacement of the 202 synthetic hydrocarbon mixture. Again, whenever necessary, individual tests involving different initial 203 concentrations of 4-chlorobenzyl alcohol and 3,4-dimethoxybenzyl alcohol were performed to achieve 204 accurate determination of the K values. All experiments were performed with 3 real replicates.

205 3. Results and Discussion

206 3.1. Preliminary partition experiments and dissolution in both phases

207 In all preliminary experiments (described in 2.3.1.) a decrease in concentration of the studied 208 compounds in the aqueous phase was observed after equilibrium contact with the model oil. It is 209 important to assess if the decrease of concentration is due to the establishment of a real solution in the 210 hydrocarbon phase. A "surfactant-like" behaviour, where the compounds concentrate at the interface 211 between phases, would also lead to a lower concentration of the compounds in the aqueous phase. 212 This type of behaviour is unwanted. Thus, samples of the hydrocarbon phase after contact with the 
213 tracer solution were taken and analysed by GC-MS (El mode). This mode of operation allows to obtain 214 qualitative information about the detected peaks. Results show that the studied compounds are 215 detected in the hydrocarbon phase. A match of the mass spectrum of $95 \%$ or greater with the library of 216 the mass spectrometer ("NIST MS search" version 2.0) was obtained for every compound (see Fig. 1 in 217 "supplementary materials). It is therefore safe to argue that the benzyl alcohols, pyrazines, and pyridine 218 in the present study form a real solution in both phases and that a real partition phenomenon was 219 observed. The study then proceeded to evaluate the influence of $\mathrm{pH}$ on the $\mathrm{K}$ value of the compounds.

\subsection{Influence of $\mathrm{pH}$ on the $\mathrm{K}$ values}

221 Possible modifications of the chemical structure of the compounds induced by changing acidity can 222 affect their partitioning behaviour. Five $\mathrm{pH}$ values between 5 and 9 were used to evaluate the $\mathrm{K}$-value 223 of the potential tracer compounds in contact with a synthetic hydrocarbon mixture (as described in 224 section 2.3.1.). Figure 2 presents the $\mathrm{K}$ values of the tested compounds $\pm \sigma(\mathrm{N}=3)$ as function of the $\mathrm{pH}$ 225 of the aqueous phase. The data about $\mathrm{pH}$ conditions available in the literature [19] suggests that the 226 range of $\mathrm{pH}$ tested is representative for most produced waters from oilfields. The primary goal of the 227 present study was to characterize the partition behaviour of the compounds it focusses on aiming for 228 their use as oil/water partitioning tracers for inter-well tests in oil reservoirs. Furthermore, the range of $229 \mathrm{pH}$ studied also provides relevant information for different disciplines where the partition behaviour of 230 the molecules in the present study plays an important role. 

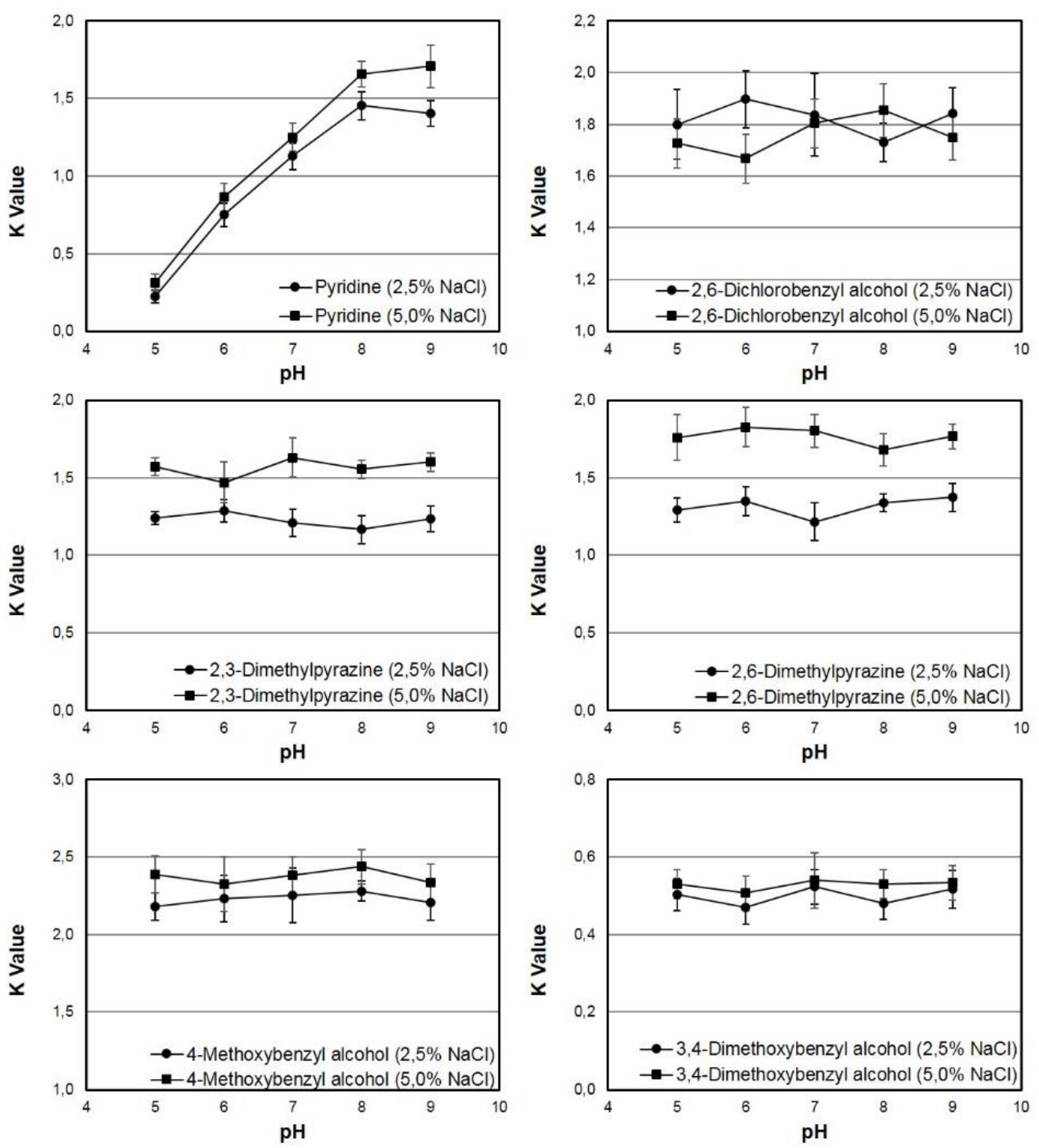

232 Fig. 1. K values of the partitioning compounds as function of $\mathrm{pH}$ at two different salinities in contact with 233 a model oil constituted by $20 \%$ (vol.) toluene, $70 \%$ (vol.) iso-octane and $10 \%$ (vol.) octanol at $25^{\circ} \mathrm{C}$. For 234 pyridine, the distribution coefficient is depicted.

235 The results depicted in Fig. 1 show that only the partitioning of pyridine is affected by $\mathrm{pH}$. The variations 236 observed for the other compounds can be attributed to the uncertainty (when precision data is 237 considered) in the experimental testing and analysis. The pKa-value of the protonated form of pyridine 238 (pyridinium) is 5,25 (see Table 1). This means that pyridinium will be the predominant species of 
239 pyridine in aqueous solution when the $\mathrm{pH}$ value is $<5,25$. As the value of $\mathrm{pH}$ increases, an equilibrium 240 between the protonated and the unprotonated forms of pyridine is established. In fact, whenever the $\mathrm{pH}$ 241 conditions of the system are such that the formation of pyridinium is significant, the observed 242 distribution value is not $K$, but rather the distribution ratio $\mathrm{D}$. $\mathrm{D}$ differs from $\mathrm{K}$ due to the fact that it 243 accounts for all dissolved species originated by the same compound dissolved in both phases [20].

$244 \quad D=\frac{C_{T(n i) O+} C_{T(i) O}}{C_{T(n i) W}+C_{T(i) W}} \quad$ (Eq. 5)

245 Where $C_{T(n i) O}$ is the concentration of non-ionic species of the compound in the hydrocarbon phase, $C_{T(i) O}$ 246 the concentration of the ionic species of the compound in the hydrocarbon, $C_{T(n i) W}$ the concentration of 247 the non-ionic species of the compound in the water phase and $C_{T(i) W}$ the concentration of the ionic 248 species of the compound in the water phase.

249 lons are not soluble in a pure hydrocarbon phase. The acid dissociation constant ( $\left.\mathrm{K}_{\mathrm{a}}\right)$ for pyridinium 250 (the protonated form of pyridine) is defined as:

$251 \quad K_{a}=\frac{[P Y R]\left[H^{+}\right]}{\left[P Y R H^{+}\right]}$

252 Eq. 6 can be rearranged and rewritten as:

$253 \frac{[P Y R]}{\left[P Y R H^{+}\right]}=10^{(p H-p K a)} \quad($ Eq. 7$)$

254 When the $\mathrm{pH}$ of the aqueous phase is close to the pKa value of pyridinium $(5,25)$, substantial amounts 255 of this substance will be present. D remains valid, however, only pyridine in its neutral form will be able 256 to dissolve in the hydrocarbon phase. At higher $\mathrm{pH}$ values, the concentration of pyridinium is orders of 257 magnitudes smaller than pyridine. Thus, acid-base equilibrium can be neglected, and the partitioning 258 behaviour of this tracer candidates becomes like all others. The partition values for pyridine at $\mathrm{pH} 8$ 259 and 9 show no trend and the variations observed become also explainable by the precision data. To 
ensure no influence of $\mathrm{pH}$ in the $\mathrm{K}$ values, the rest of the present study was performed with aqueous

261 solutions at $\mathrm{pH} 8,0$.

262 3.3. K values as function of hydrocarbon composition, salinity and temperature, and reversibility of the 263 partitioning

264 The results obtained at the experimental conditions described in 2.3.2. are presented in Fig. $2-8$. To 265 facilitate the reading of the figures, all $\mathrm{K}$ values obtained in the experiments performed with an aqueous 266 phase containing 2,5\% and 7,5\% NaCl are not depicted here in the main text. These results can, 267 however, be found in the "supplementary material" file to this article.

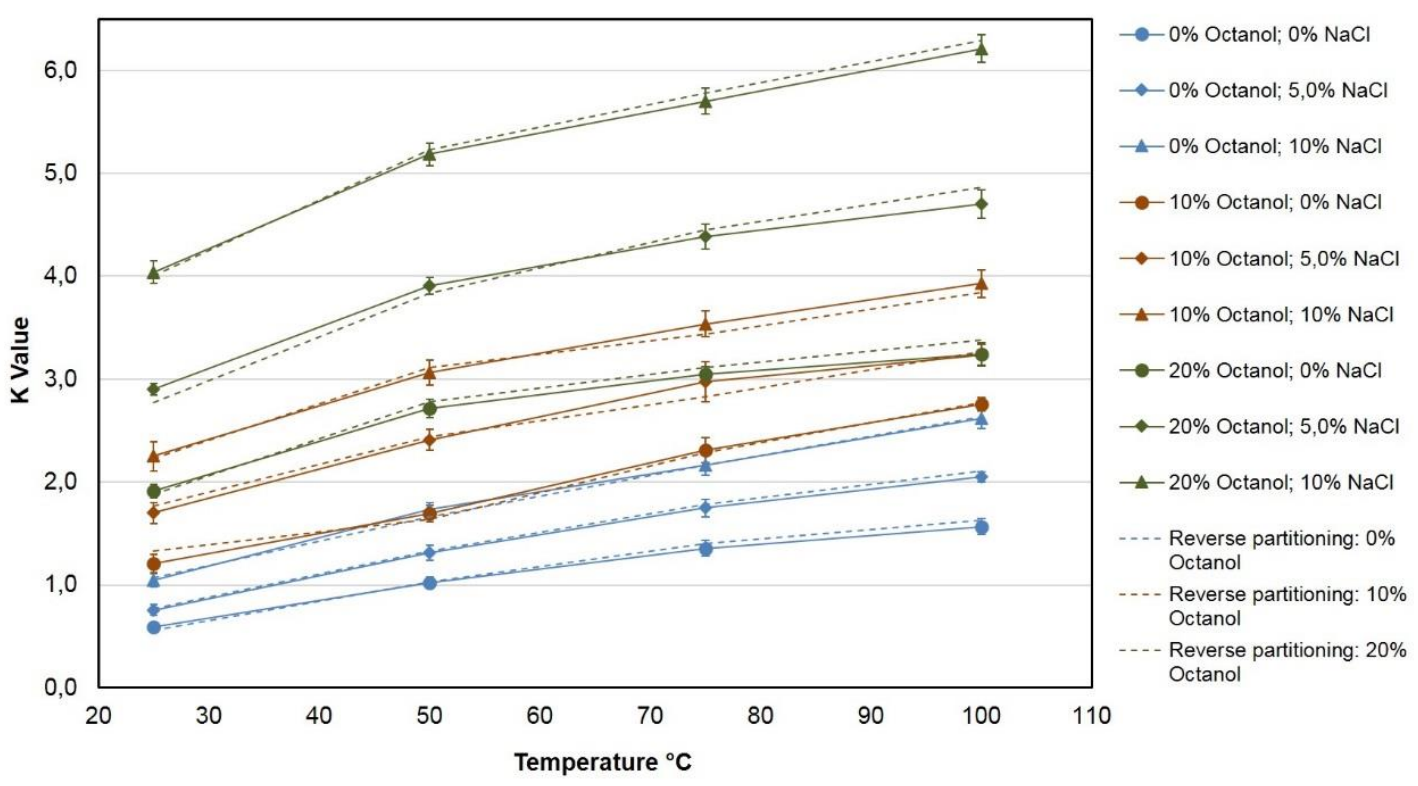

269 Fig. 2. $\mathrm{K}$ values of pyridine $\pm \sigma(\mathrm{N}=3)$ as function of temperature, composition of the hydrocarbon phase 270 and salinity (the compound is initially dissolved in the aqueous phase). The dashed lines represent the 271 K-values obtained under the same experimental conditions when the compound is initially dissolved in 272 the hydrocarbon phase.

273 The results show that the $\mathrm{K}$ value of compounds is generally influenced by all the parameters studied 274 here (oil composition, temperature, and salinity). Higher values of salinity of the aqueous phase, higher 275 temperature of the system, and increasing fraction of octanol in the hydrocarbon mixture, all cause 
276 higher partition to the hydrocarbon phase. Some exceptions are observed: temperature in the cases of 277 4-chlorobenzyl alcohol and 4-methoxybenzyl alcohol, and salinity in the case of 2,6-dichlorobenzyl 278 alcohol.

279 Temperature drives the increase of partitioning as a result of four primary effects: i) all the compounds 280 in the present study can form hydrogen bonds with water. Hydrogen bonds are strong electrostatic 281 interactions that require higher amounts of energy than dipole interactions to be broken. Increasing 282 temperatures means more energy available in the system to disturb hydrogen bonds. ii) Raoult's law 283 demonstrates that the distribution of the compounds between both phases depends on their vapor 284 pressure [21, 22], and the vapor pressure of the dissolved non-ionic compounds increases with 285 temperature. Alcohols and amines, with similar chemical structures to the molecules investigated in the 286 present work, have been reported to exhibit strong negative deviations to Raoult's law [23]. These 287 inaccurate predications can, however, be corrected by introducing compound-specific parameters to 288 account for deviations from ideal behaviour in Raoult's law equations [24] and describe accurately the 289 increase of vapor pressure as function of temperature. iii) temperature is a measure of the velocity of 290 the particles. Higher temperatures will increase the mobility of the molecules in solution, effect that 291 facilitates their diffusion to the hydrocarbon phase. iv) the dielectric constant of polar compounds (such 292 as water) varies significantly (decreases) with increasing temperatures [25]. This effect is negligible in 293 predominantly non-polar compounds (such as hydrocarbons). The dielectric constant (is a measure of 294 the ability of a chemical species to reduce the electric field between charges [26], to "insulate" them 295 from each other. This requires the existence of electrostatic interactions between the substance and the 296 ions. The dielectric constant is therefore an indirect measure of the polarity (charge distribution in the 297 structure) of a chemical species [27]. 


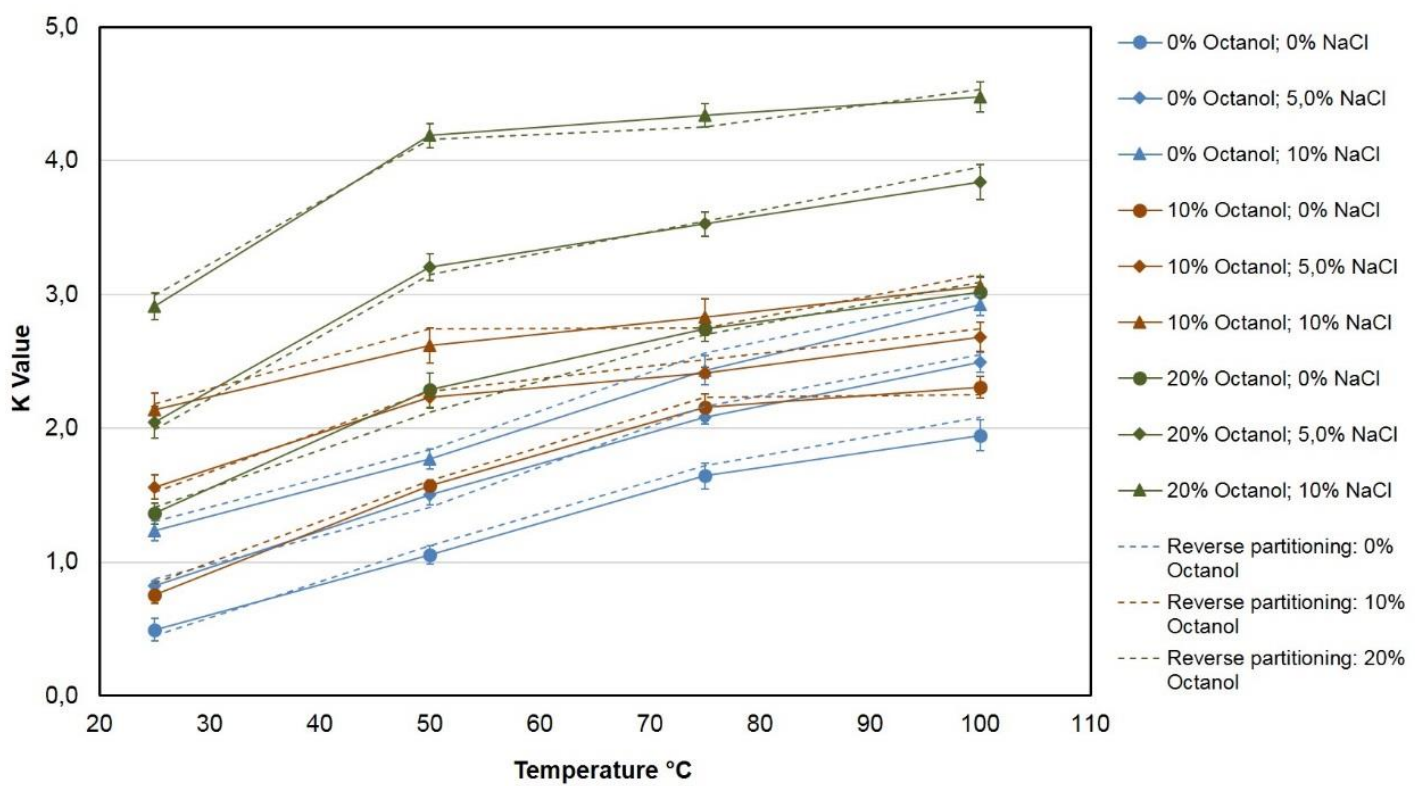

Fig. 3. $\mathrm{K}$ values of 2,3-dimethylpyrazine $\pm \sigma(\mathrm{N}=3)$ as function of temperature, composition of the hydrocarbon phase and salinity (the compound is initially dissolved in the aqueous phase). The dashed lines represent the $\mathrm{K}$ values obtained under the same experimental conditions when the compound is 302 initially dissolved in the hydrocarbon phase.

303 Consequently, increasing temperatures cause the dipole interactions between water and the 304 compounds in solution to decrease in number and intensity. The reduction of this type of interactions 305 reduces the "forces" keeping tracers in the aqueous phase. Results suggest that the global temperature 306 effect on the K-values of 4-methoxybenzyl alcohol and 4-chlorobenzyl alcohol (Fig. 6 and 8) is 307 observed only in the experiments with non-ionic aqueous phase. The extent of the influence of 308 temperature on the partitioning is dependent on the characteristics of the molecules. The most likely 309 scenario is that the temperature effect is very small compared to the variation of K caused, for example, 310 by salinity. Thus, the increase of $\mathrm{K}$ induced by temperature on 4-methoxybenzyl alcohol and 4311 chlorobenzyl alcohol is "masked" by the effect of the other parameters and appears only visible in the 312 experiments where these compounds are dissolved in deionized water. 


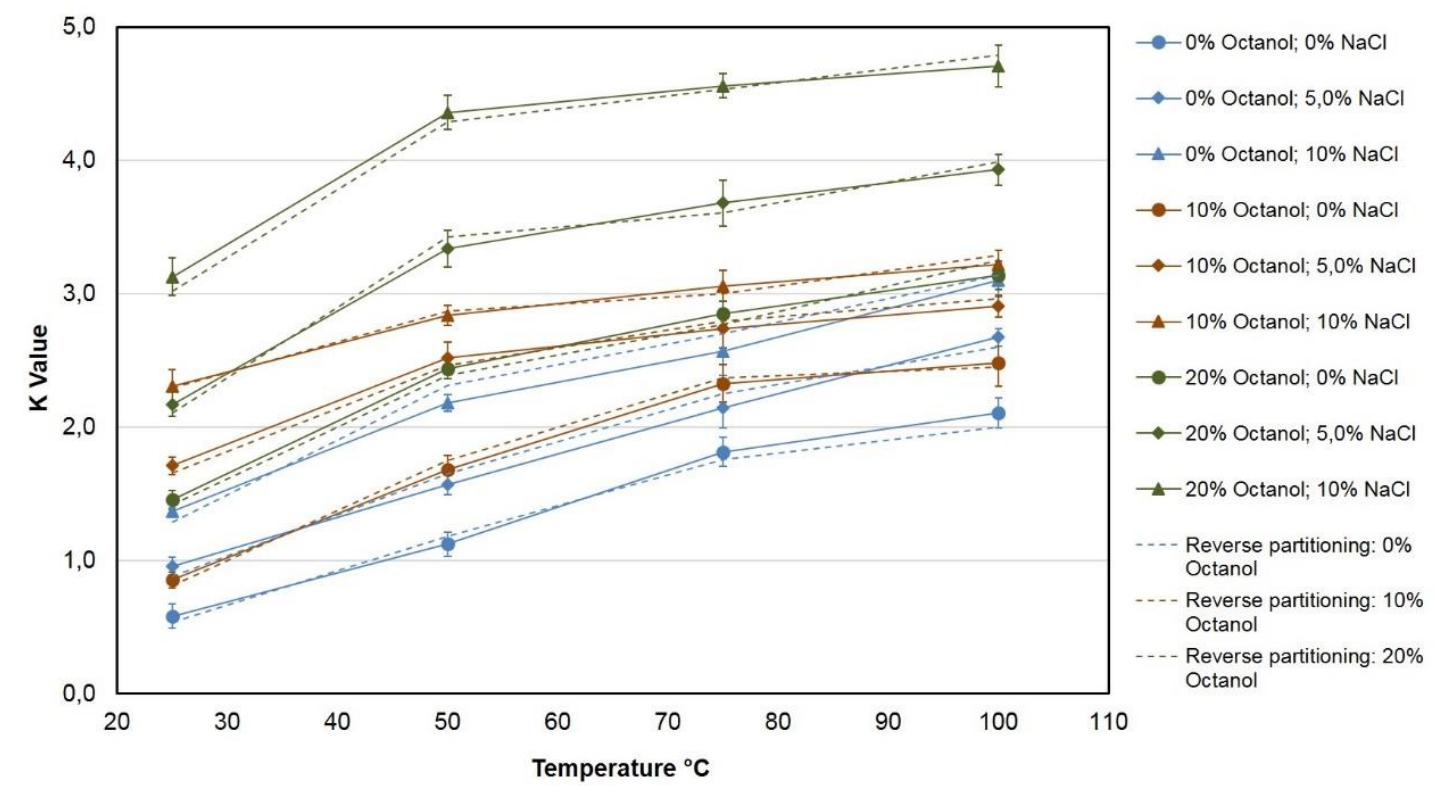

Fig. 4. $\mathrm{K}$ values of 2,6-dimethylpyrazine $\pm \sigma(\mathrm{N}=3)$ as function of temperature, composition of the hydrocarbon phase and salinity (the compound is initially dissolved in the aqueous phase). The dashed lines represent the $\mathrm{K}$ values obtained under the same experimental conditions when the compound is 317 initially dissolved in the hydrocarbon phase.

318 The $\mathrm{K}$ value of a compound is a measure of its relative solubility between a hydrocarbon and an 319 aqueous phase. Compounds which partition between such two phases are not ionized in any of the 320 solutions. Non-ionized organic compounds are dissolved in water due to polar dipole interactions and/or 321 the establishment of hydrogen bridges. The strength and number of available dipole and hydrogen 322 bridges is reduced by the presence of ions in the water. This is a well-known phenomenon that 323 generally reduces the solubility of non-ionic organic compounds in water [28, 29]. The reduction of 324 solubility of organic compounds in water caused by increasing salinity of the solution is commonly 325 called "salting-out effect". The salting-out effect is widely explored, for example, as a sample preparation technique in analytical chemistry, particularly when liquid-liquid extraction of analytes is 327 performed $[30,31]$. Increasing the amount of $\mathrm{Na}^{+}$and $\mathrm{Cl}^{-}$ions in the aqueous phase reduces the 328 solubility of molecules. Thus, more molecules will partition to the hydrocarbon phase and the $\mathrm{K}$ value will increase. The solubility of organic compounds in water has been correlated to the activity of the 
ions in solution by a proportional constant factor [28]. Results in Figures 2 - 7 suggest that such a correlation is not valid when the partition phenomena is observed. The $\mathrm{K}$ values of the potential tracer compounds under study always increase with increasing salinity. However, the results suggest that the magnitude of the variation is also dependent on the composition of the hydrocarbon phase.

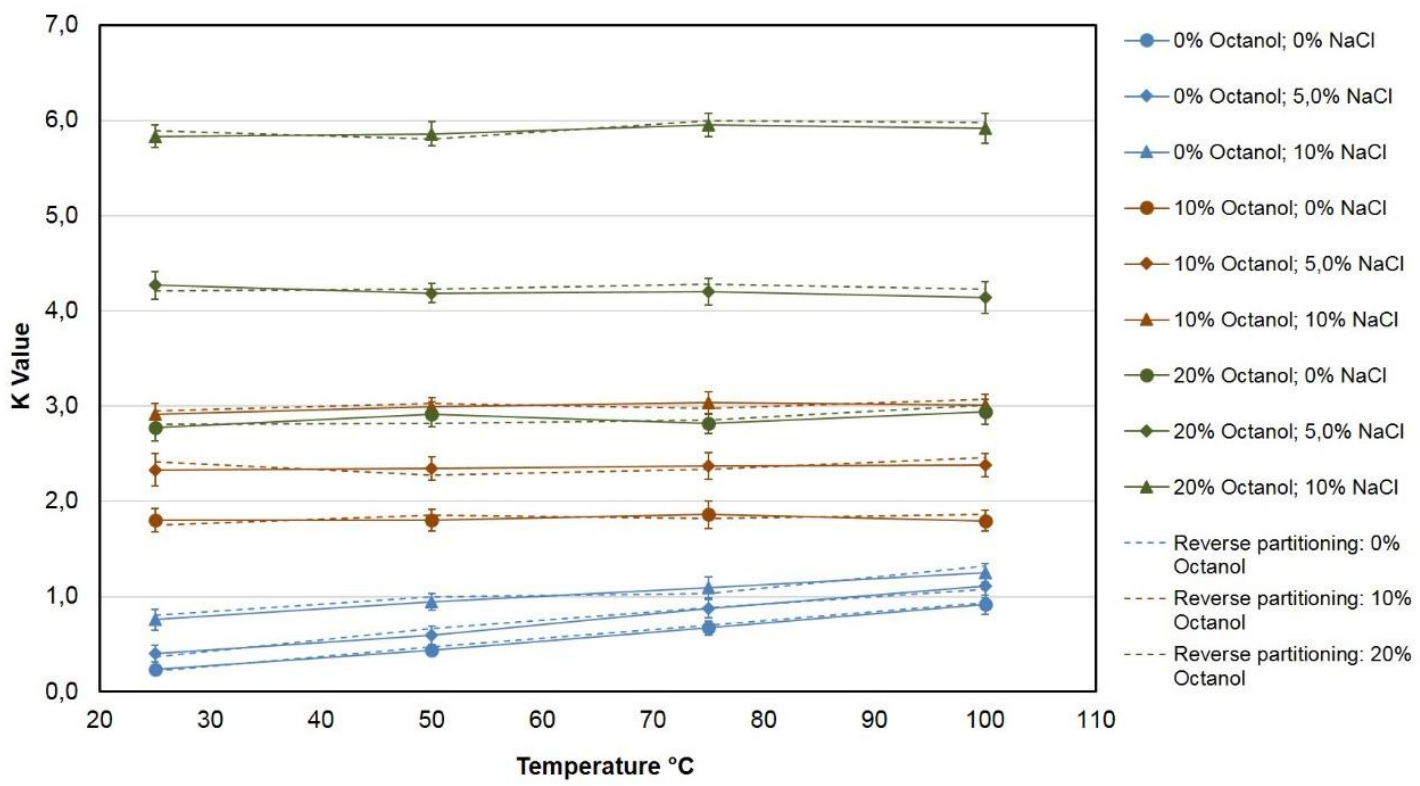

Fig. 5. $\mathrm{K}$ values of 4-methoxybenzyl alcohol $\pm \sigma(\mathrm{N}=3)$ as function of temperature, composition of the hydrocarbon phase and salinity (the compound is initially dissolved in the aqueous phase). The dashed lines represent the $\mathrm{K}$ values obtained under the same experimental conditions when the compound is initially dissolved in the hydrocarbon phase.

2,6-dichlorobenzyl alcohol (Fig. 8) shows a variation of $K$ value inversely proportional to the concentration of ions in solution ( $\mathrm{K}$ decreases with increasing salinity). No study about the solubility or partition of any benzyl alcohol as function of salinity is, to the best of our knowledge, published. While the solubility in water of non-ionic molecules generally decreases with increasing salinity, exceptions have been reported. For example, a "salting-in" effect has been described in cyclic organic compounds with chlorate and amino groups [28], and abnormal behaviour reported for benzene, naphthalene and biphenyl [32], and 1,2-benzantracene and benzo[a]pyrene [33]. A possible explanation for the "saltingin" effect observed for 2,6-dichlorobenzyl alcohol is the formation of a coordinated sphere of ions 
347 around the molecule due to its dipolar nature. 2,6-Dichlorobenzyl alcohol has 1 chlorine atom on each 348 of the carbons bounded to the carbon with the methanolic group. Both the chlorine atoms and the 349 oxygen are highly electronegative and consequently a negative dipole is induced on that side of the 350 molecule and a positive dipole on the opposite end. Cations will tend to aggregate on the negative 351 dipole side while anions will surround the molecule on the positive dipole side. Additionally, the $\pi$ 352 electrons in the benzene ring are mobile and will tend to concentrate close to the electronegative 353 elements. This effect might even be more pronounced as the concentration of positive ions increases 354 around the negative dipole, further enhancing the molecule's polar nature, and consequently increasing 355 its solubility in water. This would explain not only the decrease of $\mathrm{K}$ value with increased salinity, but 356 also higher values of $\mathrm{K}$ as function of temperature for the same salinity, as higher temperature mean 357 more movement, thus less stable electrostatic interactions. The same type of phenomena observed for 358 the partitioning of 2,6-dichlorobenzyl alcohol (Fig. 8) has been reported in liquid-liquid extraction 359 operations, as for example in the works by Makoś et al. [34, 35]. While studying the extraction of 360 aromatic and aliphatic organic acids from water, the authors verified that the yield of the process would 361 decrease with increasing $\mathrm{NaCl}$ concentrations for some of their target compounds. They offer a 362 possible explanation for this phenomenon: as the concentration of $\mathrm{NaCl}$ increases, so do the viscosity 363 and density of the solutions, leading to the formation of less efficient micro-emulsions where the mass 364 transfer occurs in the interface between the phases. This is another possible explanation for the effect 365 observed on 2,6-dichlorobenzyl alcohol. 


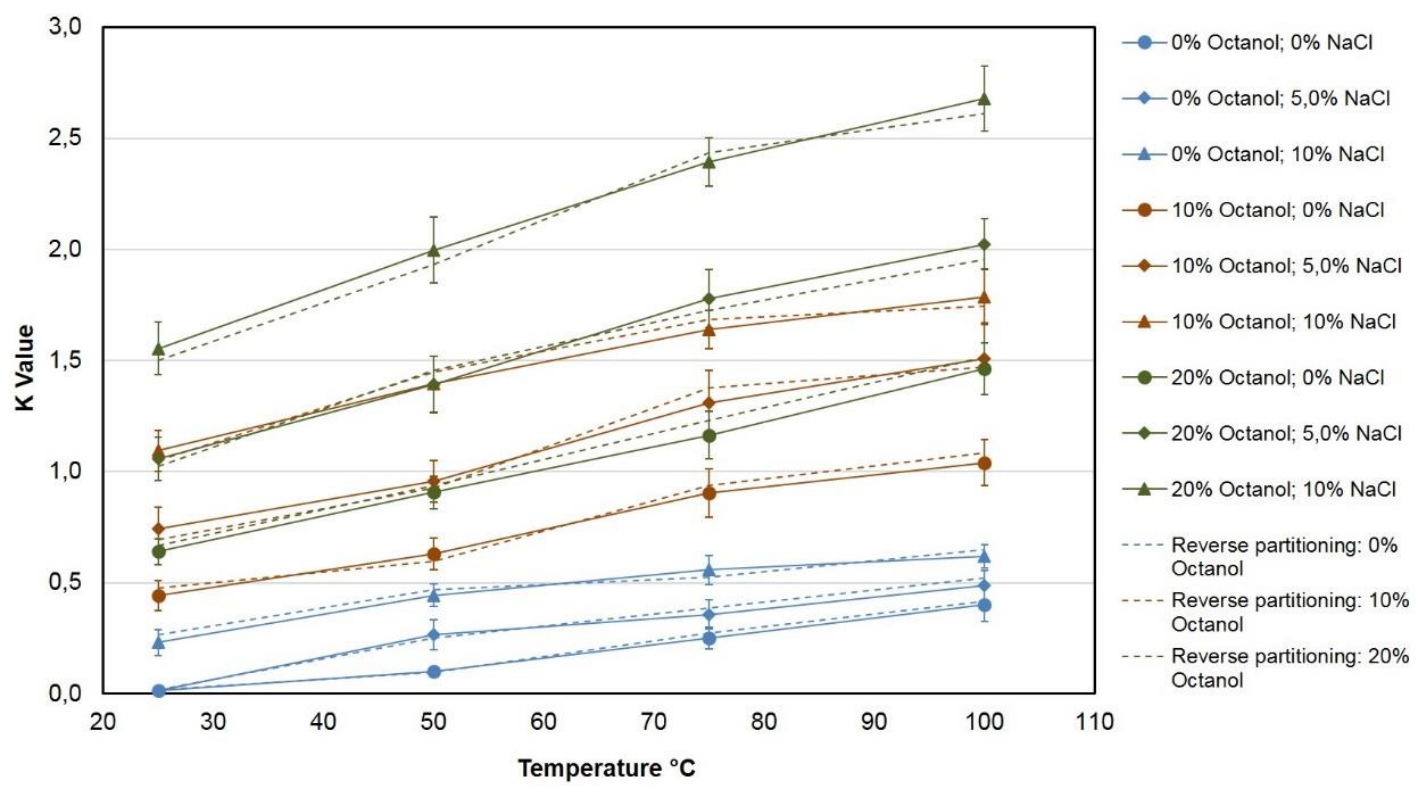

Fig. 6. $\mathrm{K}$ values of 3,4-dimethoxybenzyl alcohol $\pm \sigma(\mathrm{N}=3)$ as function of temperature, composition of the hydrocarbon phase and salinity (the compound is initially dissolved in the aqueous phase). The dashed lines represent the $\mathrm{K}$ values obtained under the same experimental conditions when the compound is initially dissolved in the hydrocarbon phase.

371 Variations on the composition of the hydrocarbon-phase also have a significant impact on the $\mathrm{K}$ value 372 of the compounds. Increasing fractions of 1-octanol generate higher $\mathrm{K}$ values. Hydrocarbons are 373 generally classified as non-polar compounds, however the relative polarities between different 374 hydrocarbons can vary significantly. Cyclic molecules, branching of the structure and increasing 375 unsaturation are known to increase the relative polarity of this class of organic compounds [36]. This 376 will naturally affect the extent of the lipophilic interaction which drives the partition process. Introducing 377 a linear saturated compound into the mixture such as 1-octanol increases the influence of lipophilic 378 interactions, such as Van der Waals forces. Furthermore, the presence of an $\mathrm{OH}$ group in the linear 379 structure induces a permanent dipole on the molecule and makes hydrogen bridges possible [37, 38]. 380 Results show clearly higher $\mathrm{K}$ values with the increase of the octanol faction in the hydrocarbon phase. 381 This is most likely due to two effects caused by the factors aforementioned: i) the establishment of 382 hydrogen bridges between the $\mathrm{OH}$ group and the molecules (all tracers studied can establish hydrogen 
bridges with the solvents); ii) a "surfactant" like alignment of the tracer molecules, that increases the 384 strength of the lipophilic forces driving then into the hydrocarbon phase.

385 The results of the experiments where the partition of the tracers occurs from the hydrocarbon to the 386 aqueous phase (dashed lines in figures $2-8$ ) show good agreement with the results of the partition 387 from the aqueous phase to the hydrocarbon phase. All the differences between the obtained $\mathrm{K}$ values 388 can be explained by the precision of the experimental methodology. Thus, it is safe to argue that the 389 partitioning of the studied candidate tracer compounds between a hydrocarbon phase and an aqueous 390 phase is constant and reversible for fixed conditions of temperature, salinity and hydrocarbon 391 composition, vary with experimental conditions and is independent of the initial concentrations of the 392 potential tracer compounds.

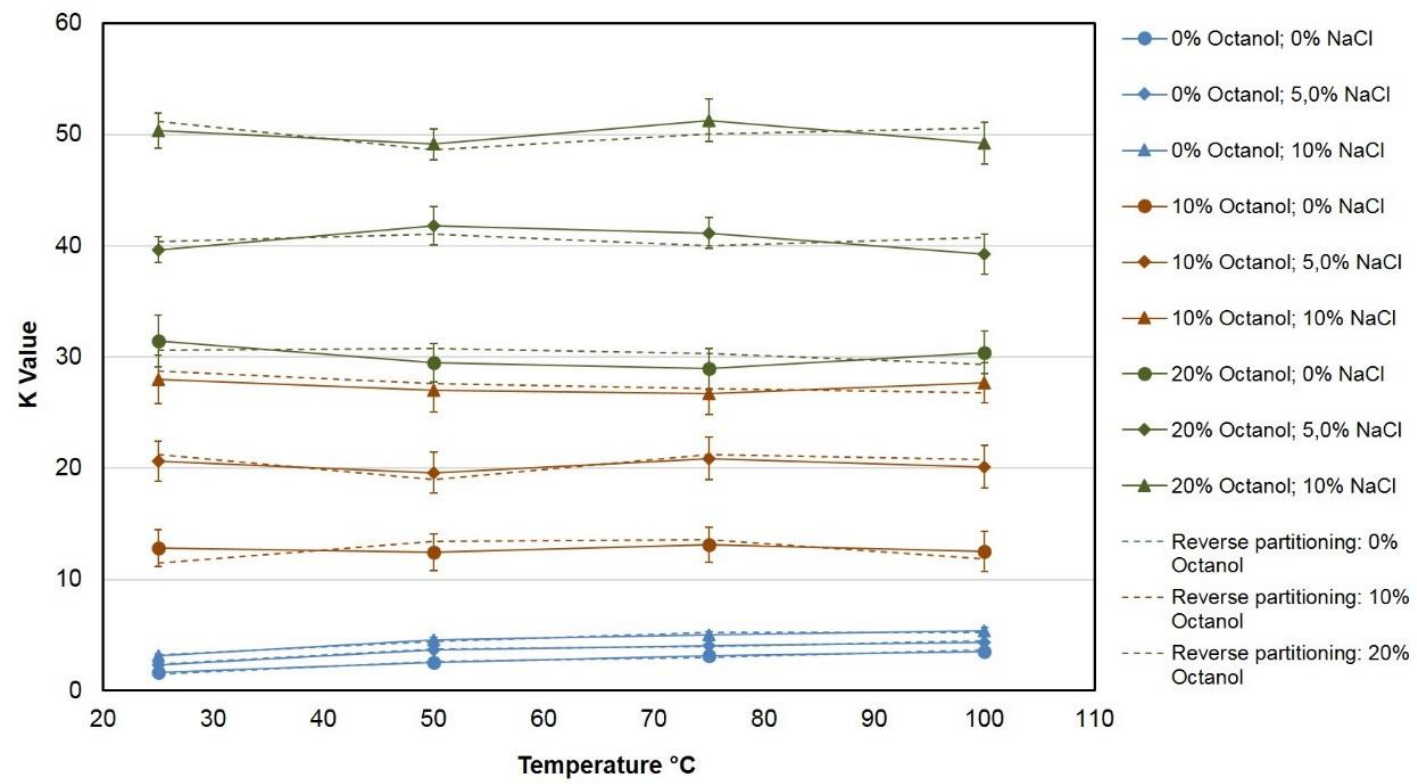

394 Fig. 7. $\mathrm{K}$ values of 4-chlorobenzyl alcohol $\pm \sigma(\mathrm{N}=3)$ as function of temperature, composition of the 395 hydrocarbon phase and salinity (the compound is initially dissolved in the aqueous phase). The dashed lines represent the $\mathrm{K}$ values obtained under the same experimental conditions when the compound is 397 initially dissolved in the hydrocarbon phase. 


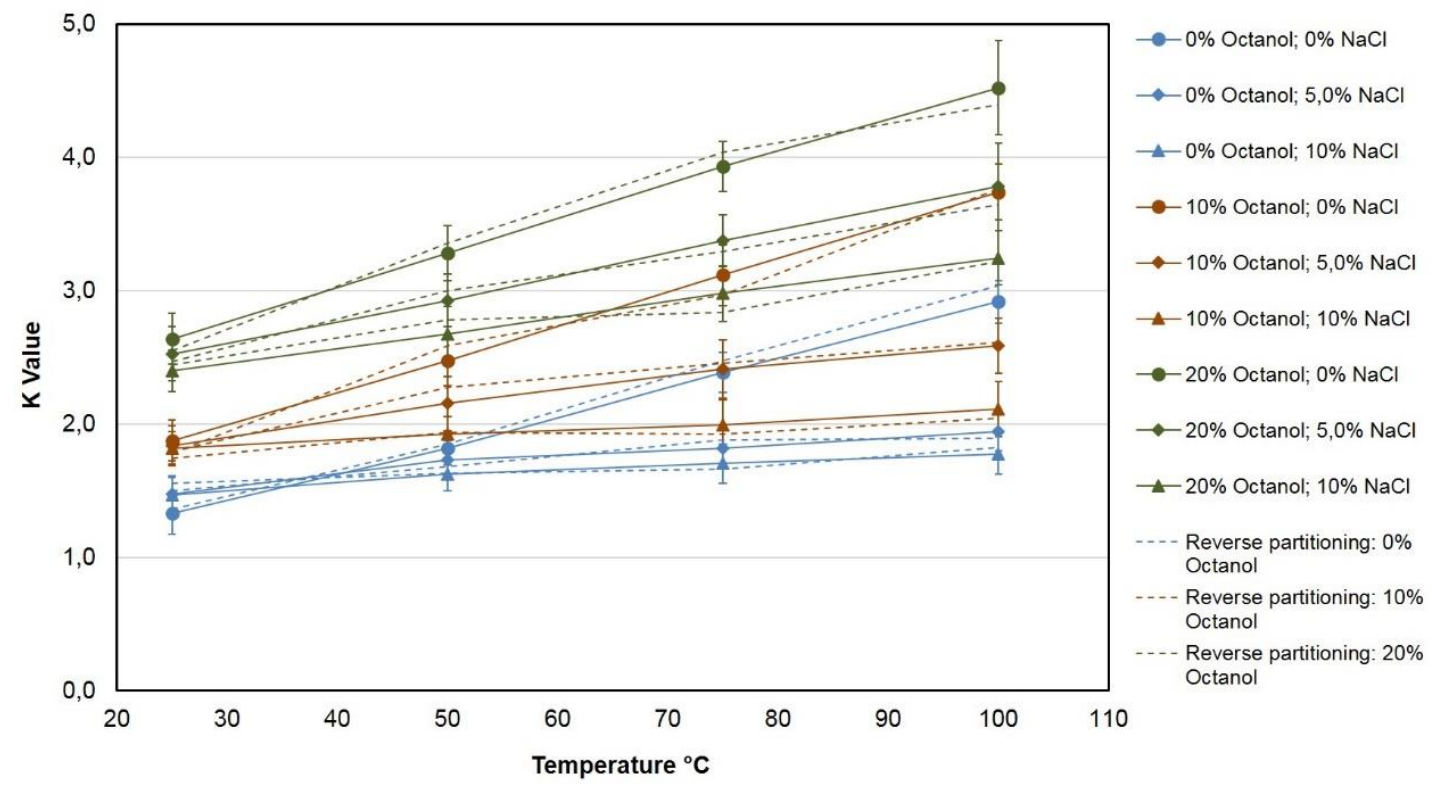

399 Fig. 8. K values of 2,6-dichlorobenzyl alcohol $\pm \sigma(N=3)$ as function of temperature, composition of the 400 hydrocarbon phase and salinity (the compound is initially dissolved in the aqueous phase). The dashed 401 lines represent the $\mathrm{K}$ values obtained under the same experimental conditions when the compound is 402 initially dissolved in the hydrocarbon phase.

403 3.4. K value as function of the presence of divalent ions in the aqueous phase

$404 \mathrm{NaCl}$ is the major salt making-up brines in oilfields. However, common oilfield brine concentrations and 405 compositions vary considerably. Other major components may be the following divalent ions: $\mathrm{Ca}^{2+}$, $406 \mathrm{Mg}^{2+}, \mathrm{Fe}^{2+}, \mathrm{Ba}^{2+}, \mathrm{CO}_{3}{ }^{2-}$ and $\mathrm{SO}_{4}{ }^{2-}$ [19]. It is therefore important to evaluate whether the electrostatic 407 interactions in the aqueous phase which influence the $\mathrm{K}$ value of the tracers are significantly changed 408 by introducing multivalent keeping the ionic strength constant. Figure 9 presents the $\mathrm{K}$ values obtained 409 in the experiments described in section 2.3.3. compared to the $\mathrm{K}$ values in experiments with the I of the 410 aqueous phase made-up of $\mathrm{NaCl}$ exclusively. The hydrocarbon phase was composed of $80 \%$ (vol) iso411 octane and $20 \%$ (vol) toluene in this case. All K values obtained for every tested compound are 412 presented. 


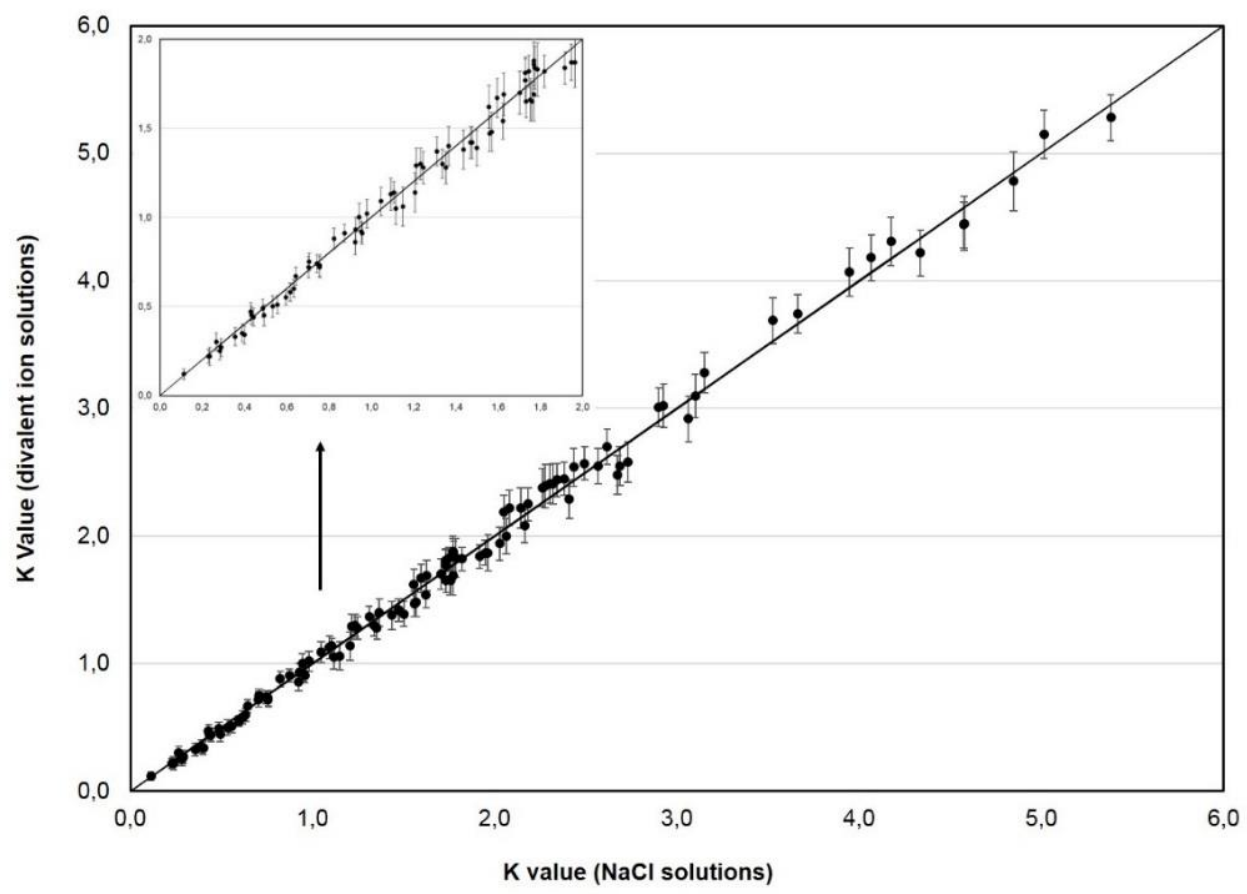

413

414 Fig. 9. $\mathrm{K}$ values $\pm \sigma(\mathrm{N}=3)$ of the tested compounds in experiments with an aqueous phase containing 415 divalent ions vs the $\mathrm{K}$ values in experiments with an aqueous phase containing only $\mathrm{NaCl}$. Hydrocarbon 416 phase composed of $80 \%$ (vol) iso-octane and $20 \%(\mathrm{vol})$ toluene. Each point obtained from the same 417 compound at the same conditions of temperature and I of the aqueous phase.

418 Results suggest that the partitioning of the tracer candidates between phases is not dependent on the 419 valency of the ions. No significant difference is encountered between the $\mathrm{K}$ values obtained in the 420 experiments where the I of the water is made-up of divalent or exclusively monovalent ions. The ionic 421 strength of the aqueous phase appears to be the relevant parameter.

$422 \quad 3.5 . \mathrm{K}$ values obtained with 5 crude oils from the Norwegian continental shelf

423 The same partition experiments as performed with synthetic hydrocarbon mixtures were carried out 424 using crude oils from the Norwegian oil fields Heidrun, Norne, Snorre, Ekofisk and Eldfisk. These are all 425 fields still in production on the Norwegian continental shelf. Figures $10-16$ present the $\mathrm{K}$ values 426 obtained for each of the tracers and oils as function of the ionic strength of the water at two different 427 temperatures $\left(50^{\circ} \mathrm{C}\right.$ and $\left.100^{\circ} \mathrm{C}\right)$. Experiments were also performed at $25^{\circ} \mathrm{C}$ and $75^{\circ} \mathrm{C}$, but the results 
are not presented here, again to facilitate the reading of the figures. The full portfolio of results are available in the table format in the "supplementary materials" file to the present document. In real field experiments with PITTs, the optimum K-value to maximize the probability of success of the test vary with expected $\mathrm{S}_{\mathrm{OR}}$ in the flooded volume between well pairs and with inter-well distance.

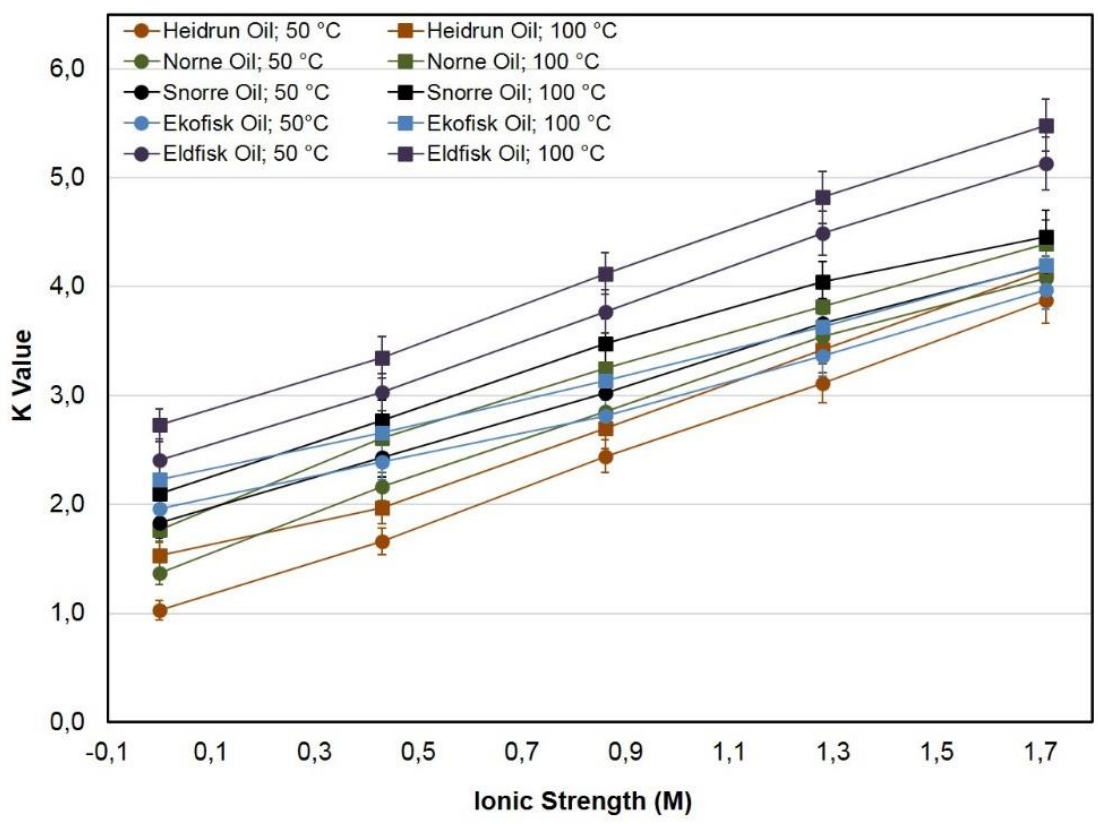

Fig. 10. $\mathrm{K}$ value of pyridine to 5 different crude oils as function of the ionic strength of the aqueous phase at $50{ }^{\circ} \mathrm{C}$ and $100^{\circ} \mathrm{C}$.

Although these limits are somewhat flexible, as they are related to the velocity of the tracers while

437 PITT. If the $\mathrm{K}$ value of the partitioning tracer is too low, there is the possibility that its production curve will overlap with the ones from the co-injected passive water tracers. This means that the difference in the arrival times of both tracer types is so small that it cannot be accurately determined. This will make the determination of $\mathrm{S}_{\mathrm{OR}}$ impossible using Eq. 3. On the other hand, if $\mathrm{K}$ is too high, the time of arrival of

441 the partitioning tracer (s) can be so long that it makes the interpretation of the test very difficult (or 442 impossible). A PITT typically takes some months to conclude. Thus, prolonging it by a poor selection of 443 the tracers used should be avoided. This can result in very flat production curves which significantly 
444 increase the difficulty of interpretation of the test because it is difficult (or impossible) to determine the 445 first moment (as a reasonable measure of the arrival time) of the production curve. $\mathrm{K}$ values in the 446 range $2-5$ are, in average, well suited for a phase-partitioning tracer to be used in a given reservoir $447[8,39]$. Thus, the goal of these experiments was to evaluate the range of conditions in which the 448 compounds in the present study could be used in the field.

449 The results from the partitioning experiments with real oils also show the influence of temperature, 450 salinity, and composition of the hydrocarbon phase in the $K$ values of the tracer candidates. 451 Furthermore, the trends produced by salinity and temperature are the same as in the experiments with 452 the model oils. This suggests that the influence of these two parameters, although "buffered" by the 453 hydrocarbon phase, is intimately dependent on the chemical properties of the molecules.

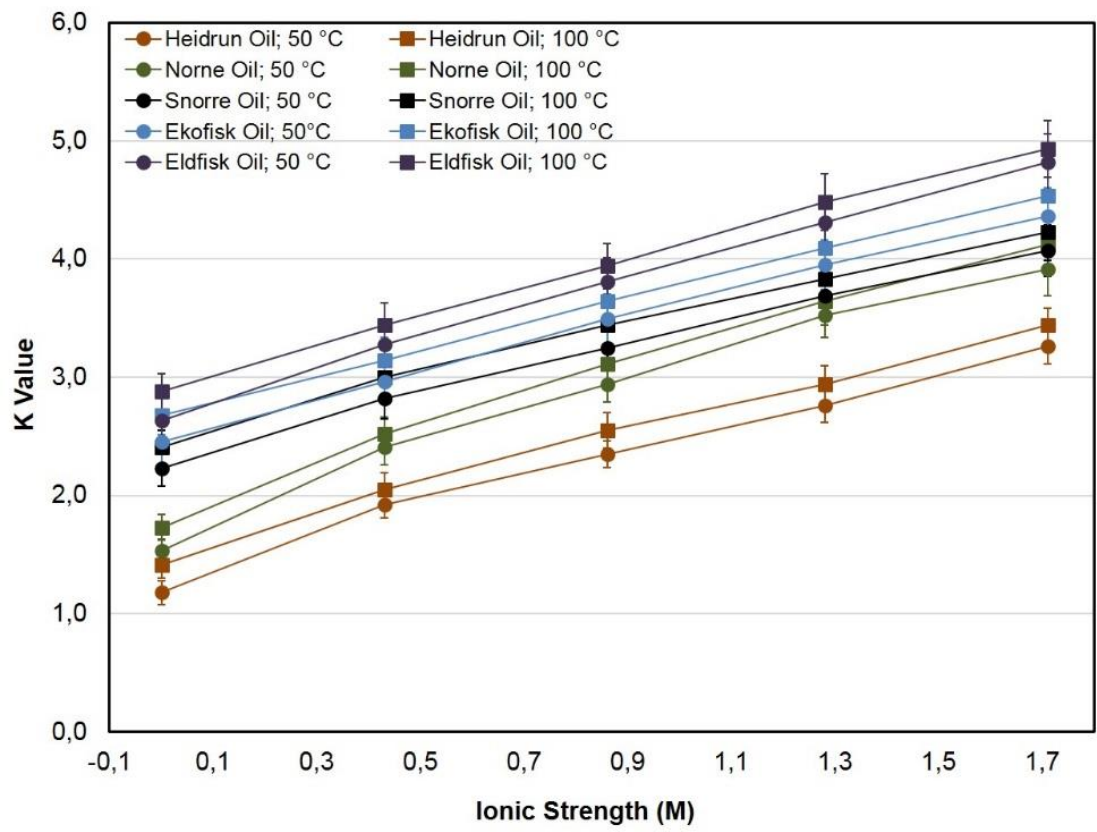

455 Fig. 11. K value of 2,3-dimethylpyrazine to 5 different crude oils as function of the ionic strength of the 456 aqueous phase at $50^{\circ} \mathrm{C}$ and $100^{\circ} \mathrm{C}$. 


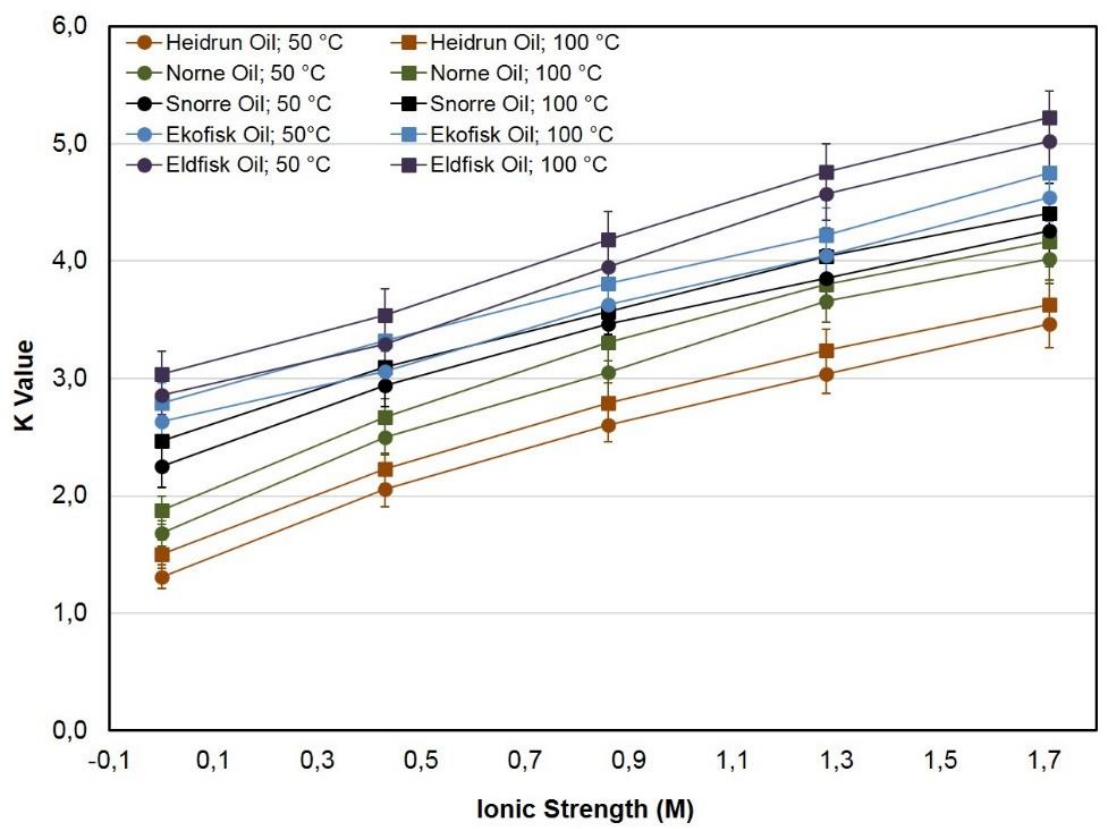

457

458 Fig. 12. $\mathrm{K}$ value of 2,6-dimethylpyrazine to 5 different crude oils as function of the ionic strength of the 459 aqueous phase at $50^{\circ} \mathrm{C}$ and $100^{\circ} \mathrm{C}$.

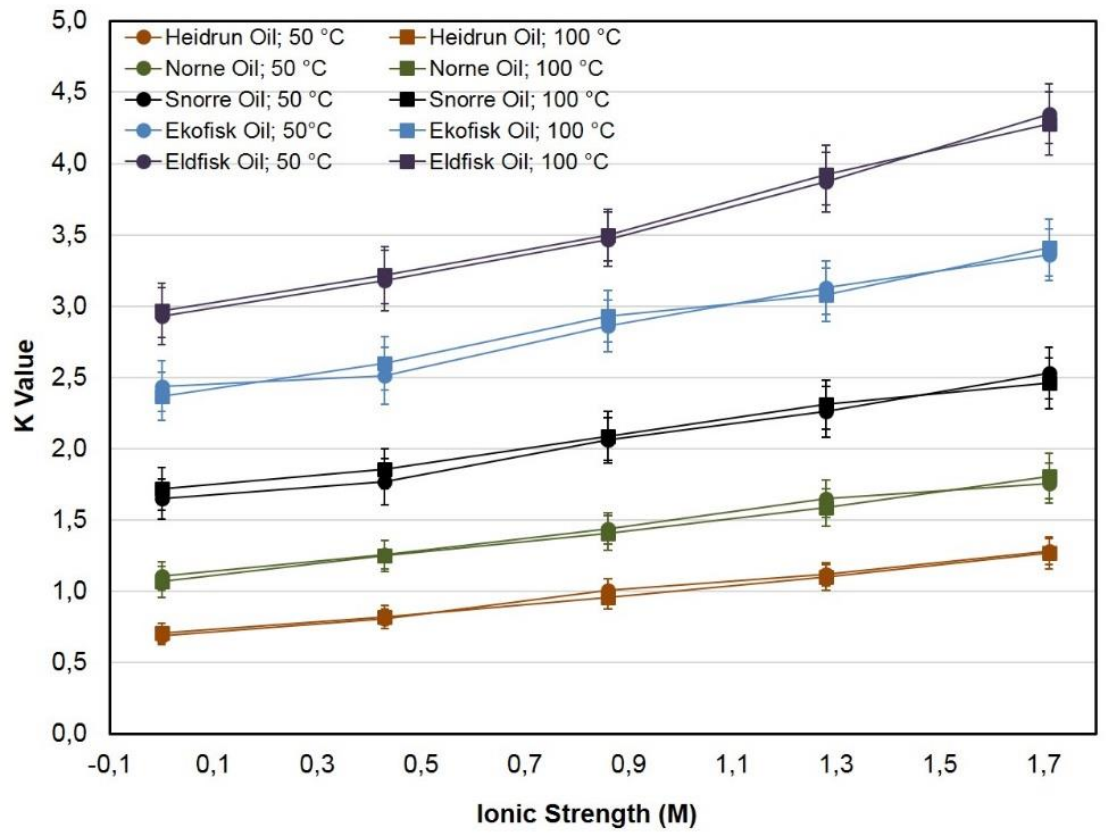

461 Fig. 13. K value of 4-methoxybenzyl alcohol to 5 different crude oils as function of the ionic strength of 462 the aqueous phase at $50^{\circ} \mathrm{C}$ and $100^{\circ} \mathrm{C}$. 


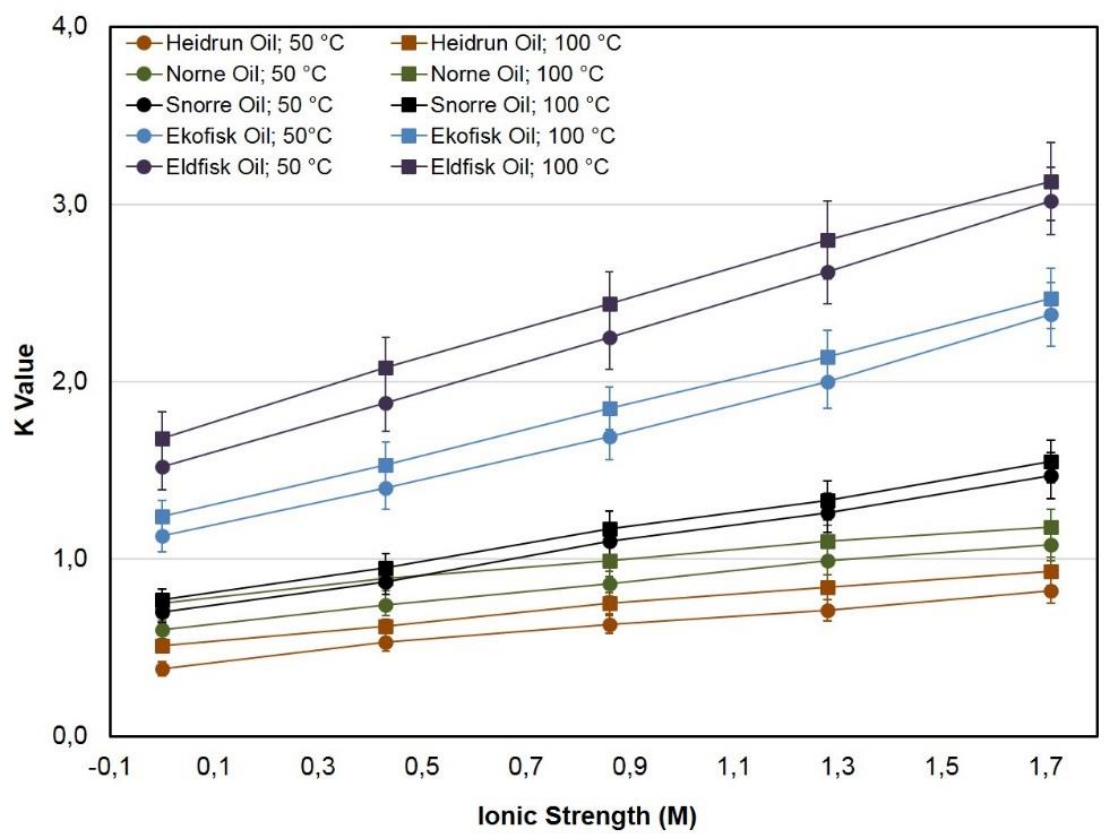

464 Fig. 14. K value of 3,4-dimethoxybenzyl alcohol to 5 different crude oils as function of the ionic strength 465 of the aqueous phase at $50{ }^{\circ} \mathrm{C}$ and $100{ }^{\circ} \mathrm{C}$.

466 The range of variation of the $\mathrm{K}$ values of pyridine is similar to that observed in the experiments with 467 model oil. Most of the $\mathrm{K}$ values obtained are well within the desired range. This suggests that pyridine 468 would perform well as a phase-partitioning tracer for PITTs. 2,3-dimethylpyrazine and 2,6469 dimethylpyrazine exhibit very similar $\mathrm{K}$ values for the same systems. This was also observed in the 470 experiments with the synthetic hydrocarbon mixture. The similarity between these molecules (positional 471 isomers) explains the small difference in $\mathrm{K}$ values, as the physico-chemical properties of both 472 molecules are very similar. Generally, 2,3-dimethylpyrazine and 2,6-dimethylpyrazine also exhibit K 473 values to the stock tank oil samples that suggest that these are promising compounds for use as PITT 474 tracers. 


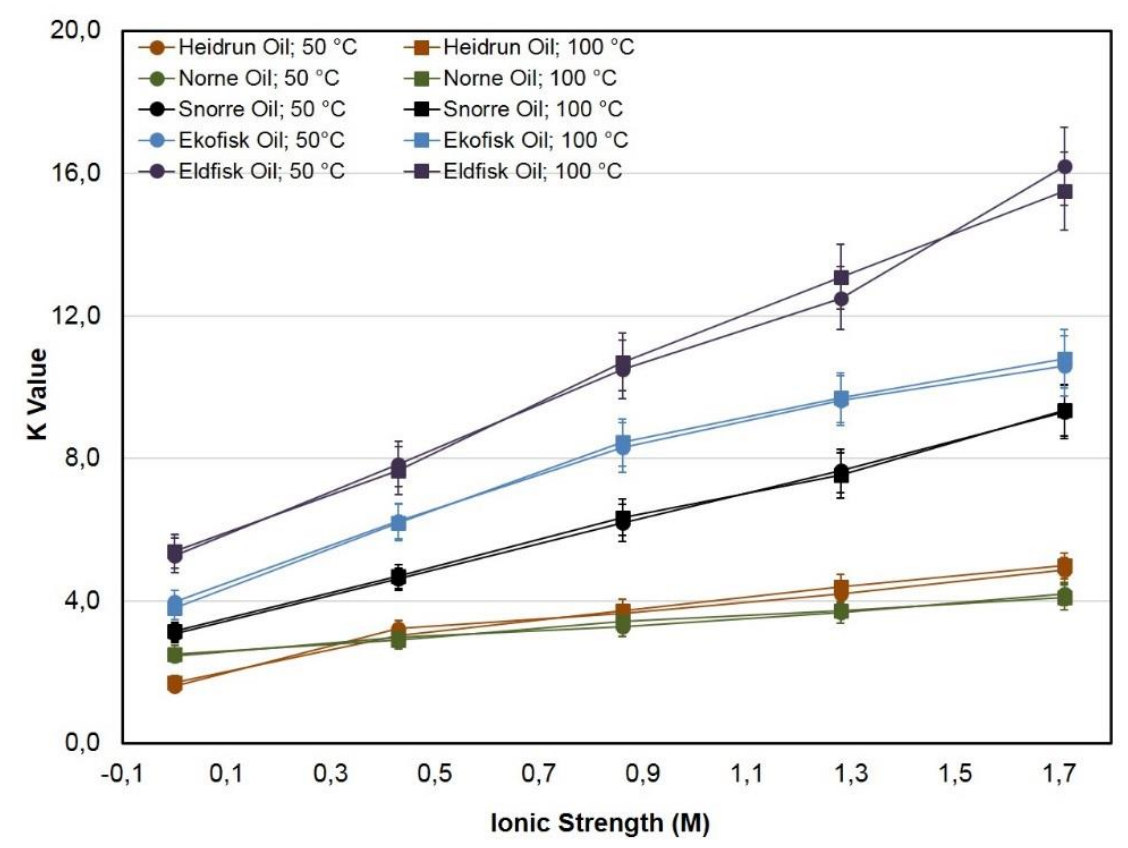

475

Fig. 15. K value of 4-chlorobenzyl alcohol to 5 different crude oils as function of the ionic strength of the aqueous phase at $50^{\circ} \mathrm{C}$ and $100^{\circ} \mathrm{C}$.

478 The partitioning of 4-methoxybenzyl alcohol to the tested stock tank oils is again independent of 479 temperature (Fig. 13). This was also observed in the experiments with the model oil mixture of hydrocarbons whenever 1-octanol was one of the constituents. The $\mathrm{K}$ values of 4-methoxybenzyl 481 alcohol vary little as function of salinity for each type of oil tested and are systematically below 2 for 482 Heidrun and Norne oils. As a result of the low K values observed, the use of 4-methoxybenzyl alcohol 483 as a PITT tracer is more appropriate in fields with long distances between injection/producer pairs or 484 when high values of Sor are expected. Results show that 3,4-dimethoxybenzyl alcohol (Fig. 14) is the 485 most hydrophilic of all the tested compounds. Most of the K values with real stock tank oils are below 2. 486 This also makes the field use of 3,4-dimethoxybenzyl alcohol more appropriate for large distances or 487 higher oil saturations between well pairs. The partitioning of 4-chlorobenzyl alcohol to crude oils agrees 488 with the ones obtained with the model oils when it comes to the influence of temperature. The $\mathrm{K}$ values 489 of this compound is independent of temperature. 4-chlorobenzyl alcohol exhibits the higher $\mathrm{K}$ values 490 (Fig. 15) to the tested oils. In addition, it shows a higher variation of $\mathrm{K}$ as function of salinity (ionic 
491 strength) of the aqueous phase. It is the most lipophilic molecule tested and its use as PITT tracer 492 seems more appropriate for short distances between injection and production well pairs or in situations 493 with expected low $S_{O R}$ in the reservoir. The K values of 2,6-dichlorobenzyl alcohol (Fig. 17) vary with 494 the inverse of the ionic strength. Like in the experiments with the model oil mixtures, the $\mathrm{K}$ value of this 495 compound decreases as function of I of the aqueous phase for all the oil types tested. Nevertheless, 496 the range of $\mathrm{K}$ values obtained suggests that 2,6-dichlorobenzyl alcohol is a tracer with a wide range of 497 applications. The decrease of $\mathrm{K}$ as function of the salinity of the water might make this compound 498 particularly interesting for very high salinity reservoirs, where the $\mathrm{K}$ values of other phase-partitioning 499 tracers might increase to such values that the test simply becomes too time consuming.

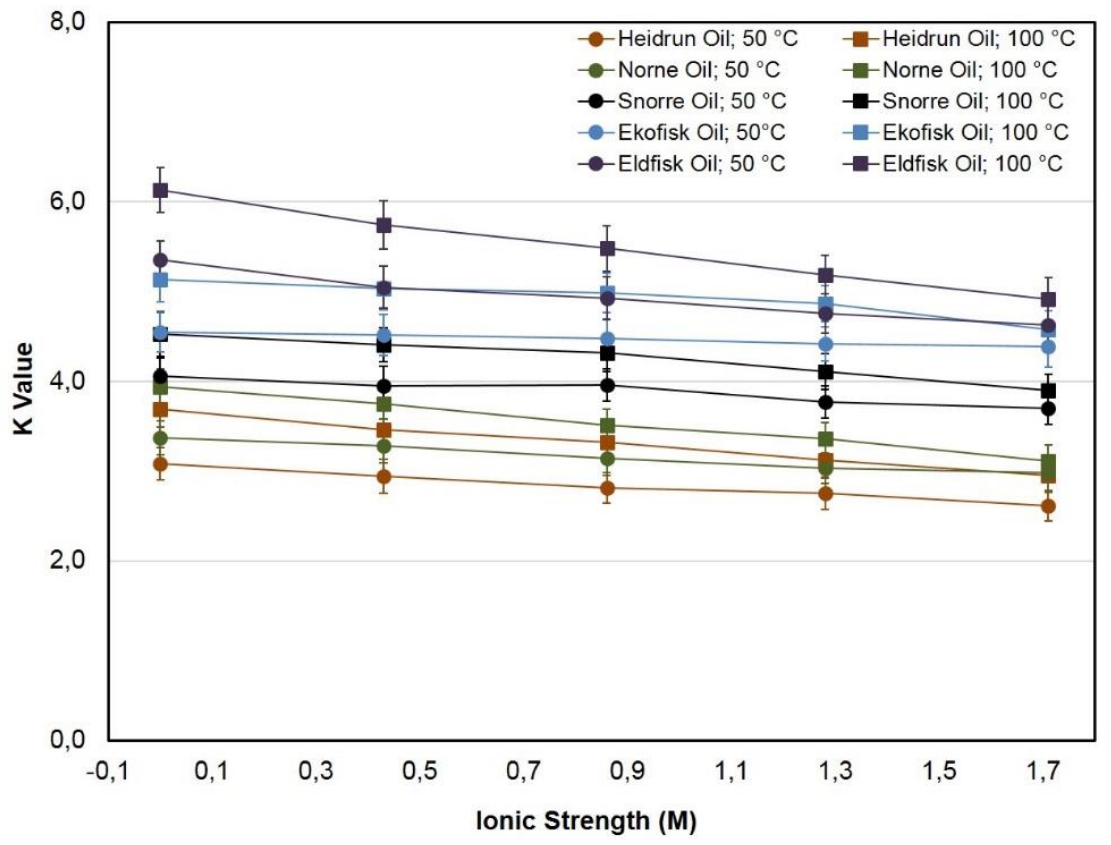

501 Fig. 16. K value of 2,6-dichlorobenzyl alcohol to 5 different crude oils as function of the ionic strength of 502 the aqueous phase at $50^{\circ} \mathrm{C}$ and $100^{\circ} \mathrm{C}$.

503 4. Conclusions

504 The partition coefficients between water and oil of pyridine, 2,3-dimethylpyrazine, 2,6-dimethylpyrazine, 505 4-methoxybenzyl alcohol, 3,4-dimethoxybenzyl alcohol, 4-chlorobenzyl alcohol and 2,6-dichlorobenzyl 
506 alcohol were determined as function of the hydrocarbon phase composition, temperature of the system 507 and the $\mathrm{pH}$, salinity (ionic strength) and ionic composition of the aqueous phase. $\mathrm{K}$ values were also 508 determined with 5 different real black (stock tank) oils from different reservoirs at the Norwegian 509 continental shelf.

510 All the tested compounds were found to form real solutions in the hydrocarbon phase. The phase 511 partitioning of all the compounds was found to be constant and reversible for system with fixed 512 properties of temperature, salinity of the aqueous phase and hydrocarbon composition. The presence 513 of aliphatic organic molecules containing hydrophilic groups $(\mathrm{OH})$ was found to increase the partitioning 514 of every compound to the hydrocarbon phase. This effect is less pronounced for 3,4-dimethoxybenzyl 515 and more pronounced for 4-chlorobenzyl alcohol. Increasing temperatures also increase the $\mathrm{K}$ values 516 of the molecules, except in the cases of 4-chlorobenzyl alcohol and 4-methoxybenzyl alcohol. In the 517 case of these compounds, the temperature effect is only visible when the hydrocarbon phase is 518 primarily constituted by aromatic organics. Results show that $\mathrm{pH}$ only influences the partitioning of 519 pyridine to the hydrocarbon phase. This is the result of the basic properties of this compound that 520 cause it to remain ionized at the lower $\mathrm{pH}$ values. If used together with $\mathrm{pH}$ insensitive tracers, pyridine 521 might be used to retrieve real $\mathrm{pH}$ data from systems where it is used as tracer. Increasing the ionic 522 strength of the aqueous phase reduces the water solubility of all the tested molecules, increasing their 523 partition to the hydrocarbon phase, except for 2,6-dichlorobenzyl alcohol. The $\mathrm{K}$ value of this compound 524 is reduced by increasing salinity in the range of ionic strength tested. The presence of divalent ions 525 composing the aqueous phase was found to have no visible effect on the partitioning of the 526 compounds. The ionic strength of the solution appears as the predominant parameter driving the 527 partitioning. Pyridine, 2,3-dimethylpyrazine, 2,6-dimethylpyrazine and 2,6-dichlorobenzyl alcohol exhibit $528 \mathrm{~K}$ values, as function of the tested parameters, within ranges that suggest they are appropriate for use 529 as PITT tracers in most oilfields. The range of $\mathrm{K}$ values to real oils obtained for 4-methoxybenzyl 530 alcohol and 3,4-dimethoxybenzyl alcohol indicates that these compounds are more appropriate for 531 PITT on large distances between well-pairs or in volumes with expected high $\mathrm{S}_{\mathrm{OR}}$ values. The use of 4- 
532 chlorobenzyl alcohol as PITT tracer is more appropriate for short distances between well-pairs or 533 expected low values of Sor in the flow paths.

\section{5. Acknowledgements}

535 The authors acknowledge the Research Council of Norway and the industry partners, ConocoPhillips 536 Skandinavia AS, Aker BP ASA, Vår Energi AS, Equinor ASA, Neptune Energy Norge AS, Lundin 537 Norway AS, Halliburton AS, Schlumberger Norge AS, Wintershall Norge AS, and DEA Norge AS, of 538 The National IOR Centre of Norway for support.

539 6. References

540 [1] IEA. World Energy Outlook 2019. Paris: IEA; 2019.

541 [2] Silva M, Stray H, Bjørnstad T. Stability assessment of PITT tracer candidate compounds - The case of $542 \quad$ pyrazines. Journal of Petroleum Science and Engineering 2019;182:106269.

543 [3] Cooke CEJ. "Method of determining fluid saturations in reservoirs" (US Patent 3,590,923). US Patent $544 \quad 3,590,923$. USA: Esso Production Research Company; 1971.

545 [4] Tang JS. Interwell Tracer Tests To Determine Residual Oil Saturation To Waterflood At Judy Creek 546 Bhl'a'pool. PETSOC-91-04-01 1992;31(08):12.

547 [5] Deans HA. Using Chemical Tracers To Measure Fractional Flow And Saturation In-Situ. SPE Symposium on 548 Improved Methods of Oil Recovery. Tulsa, Oklahoma: Society of Petroleum Engineers; 1978:10.

549 [6] Tang JS. Partitioning Tracers and In-Situ Fluid Saturation Measurements. SPE-22344-PA 1995;10(01):33 $550 \quad 9$.

551 [7] Sanni M, Al-Abbad M, Kokal S, Dugstad $\varnothing$, Hartvig S, Huseby O. Pushing the envelope of residual oil 552 measurement: A field case study of a new class of inter-well chemical tracers. Journal of Petroleum Science and Engineering 2018;163:538-45. 
554 [8] Viig SO, Juilla H, Renouf P, Kleven R, Krognes B, Dugstad O, et al. Application of a New Class of Chemical Tracers To Measure Oil Saturation in Partitioning Interwell Tracer Tests. SPE International Symposium on Oilfield Chemistry. The Woodlands, Texas, USA: Society of Petroleum Engineers; 2013.

557 [9] Wood KN, Tang S, Luckasavitch RJ. Interwell Residual Oil Saturation at Leduc Miscible Pilot. SPE Annual Technical Conference and Exhibition. New Orleans, Louisiana: Society of Petroleum Engineers; 1990.

559 [10] Ratnakar RR, Dindoruk B, Wilson L. Experimental investigation of DME-water-crude oil phase behavior and PVT modeling for the application of DME-enhanced waterflooding. Fuel 2016;182:188-97.

561

[11] Zhang G, Yu J. Effect of commonly used EOR polymers on low concentration surfactant phase behaviors. Fuel 2021;286:119465.

[12] Moradi M, Topchiy E, Lehmann TE, Alvarado V. Impact of ionic strength on partitioning of naphthenic acids in water-crude oil systems - Determination through high-field NMR spectroscopy. Fuel 2013;112:236-48.

566

[13] Leo A, Hansch C, Elkins D. Partition coefficients and their uses. Chemical Reviews 1971;71(6):525-616.

[14] Dearden JC, Bresnen GM. The Measurement of Partition Coefficients. Quantitative Structure-Activity Relationships 1988;7(3):133-44.

[15] Ramachandran SD, Sweezey MJ, Hodson PV, Boudreau M, Courtenay SC, Lee K, et al. Influence of salinity and fish species on PAH uptake from dispersed crude oil. Marine Pollution Bulletin 2006;52(10):1182-9.

571 [16] Salager J-L, Marquez N, Graciaa A, Lachaise J. Partitioning of Ethoxylated Octylphenol Surfactants in Microemulsion-Oil-Water Systems: Influence of Temperature and Relation between Partitioning Coefficient and Physicochemical Formulation. Langmuir 2000;16(13):5534-9.

574 [17] Stephan C, Dicko M, Stringari P, Coquelet C. Liquid-liquid equilibria of water + solutes (acetic acid/ 575 576 acetol/furfural/guaiacol/methanol/phenol/propanal) + solvents (isopropyl acetate/toluene) ternary systems for pyrolysis oil fractionation. Fluid Phase Equilibria 2018;468:49-57. 
577 [18] Silva M, Bjørnstad T. Determination of phase-partitioning tracer candidates in production waters from oilfields based on solid-phase microextraction followed by gas chromatography-tandem mass spectrometry. Journal of Chromatography A 2020;1629:461508.

[19] Fakhru'I-Razi A, Pendashteh A, Abdullah LC, Biak DRA, Madaeni SS, Abidin ZZ. Review of technologies for oil and gas produced water treatment. Journal of Hazardous Materials 2009;170(2):530-51.

[20] Sangster J. Octanol-Water Partition Coefficients of Simple Organic Compounds. Journal of Physical and Chemical Reference Data 1989;18(3):1111-229.

584 [21] Cline PV, Delfino JJ, Rao PSC. Partitioning of aromatic constituents into water from gasoline and other complex solvent mixtures. Environmental Science \& Technology 1991;25(5):914-20.

[22] Chen CS, Lai Y-W, Tien C-J. Partitioning of aromatic and oxygenated constituents into water from regular and ethanol-blended gasolines. Environmental Pollution 2008;156(3):988-96.

[23] González JA, de la Fuentá IG, Cobos JC. Thermodynamics of mixtures with strongly negative deviations 589 from Raoult's Law: Part 4. Application of the DISQUAC model to mixtures of 1-alkanols with primary or secondary linear amines. Comparison with Dortmund UNIFAC and ERAS results. Fluid Phase Equilibria 2000;168(1):31-58.

Endo S, Schmidt TC. Prediction of Partitioning between Complex Organic Mixtures and Water: Application of Polyparameter Linear Free Energy Relationships. Environmental Science \& Technology 2006;40(2):536-45.

Hawthorne SB, Yang Y, Miller DJ. Extraction of Organic Pollutants from Environmental Solids with Suband Supercritical Water. Analytical Chemistry 1994;66(18):2912-20.

Pinkert A, Marsh KN, Pang S, Staiger MP. Ionic Liquids and Their Interaction with Cellulose. Chemical Reviews 2009;109(12):6712-28. 
599 [27] Griffiths TR, Pugh DC. Correlations among solvent polarity scales, dielectric constant and dipole moment, and a means to reliable predictions of polarity scale values from cu. Coordination Chemistry Reviews 601 1979;29(2):129-211.

602 [28] Xie W-H, Shiu W-Y, Mackay D. A review of the effect of salts on the solubility of organic compounds in 603 604

[29] Benbouzid H, Le Floch S, Stephan L, Olier R, Privat M. Combined effects of salinity and temperature on 605 the solubility of organic compounds. The Journal of Chemical Thermodynamics 2012;48:54-64.

606

[30] Tang YQ, Weng N. Salting-out assisted liquid-liquid extraction for bioanalysis. Bioanalysis 607 2013;5(12):1583-98.

[31] Anthemidis AN, loannou K-IG. Recent developments in homogeneous and dispersive liquid-liquid extraction for inorganic elements determination. A review. Talanta 2009;80(2):413-21. Janado M, Yano Y, Doi Y, Sakamoto H. Peculiar effects of alkali thiocyanates on the activity coefficients

[34] Makoś P, Fernandes A, Przyjazny A, Boczkaj G. Sample preparation procedure using extraction and derivatization of carboxylic acids from aqueous samples by means of deep eutectic solvents for gas chromatographic-mass spectrometric analysis. Journal of Chromatography A 2018;1555:10-9. polycyclic aromatic hydrocarbons in aqueous samples. Journal of Chromatography A 2018;1570:28-37. Hydrocarbons1. The Journal of Physical Chemistry 1966;70(4):1267-75.

Xing B, McGill WB, Dudas MJ. Cross-Correlation of Polarity Curves To Predict Partition Coefficients of 
623 [38] Sprakel LMJ, Schuur B. Solvent developments for liquid-liquid extraction of carboxylic acids in 624 perspective. Separation and Purification Technology 2019;211:935-57.

625 [39] Silva M, Stray H, Bjørnstad T. Studies on New Chemical Tracers for Determination of Residual Oil 626 Saturation in the Inter-Well Region. SPE Oklahoma City Oil and Gas Symposium. Oklahoma City, $627 \quad$ Oklahoma, USA: Society of Petroleum Engineers; 2017. 